\title{
Hodge correlators
}

\author{
A.B. Goncharov \\ To Alexander Beilinson for his 50th birthday
}

\section{Contents}

1 Introduction

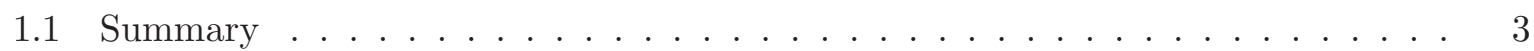

1.2 Arithmetic motivation I: special values of $L$-functions $\ldots \ldots \ldots \ldots$

1.3 Arithmetic motivation II: arithmetic analysis of periods . . . . . . . . . . 6

1.4 Mixed motives and the motivic Lie algebra . . . . . . . . . . . . . . 9

1.5 Pronilpotent completions of fundamental groups of curves . . . . . . . . . . . 10

1.6 Hodge correlators . . . . . . . . . . . . . . . . . . . . . . . . . . . . . . . . . . . . .

1.7 A Feynman integral for Hodge correlators . . . . . . . . . . . . . . . . . 13

1.8 Variations of mixed $\mathbb{R}$-Hodge structures by twistor connections . . . . . . . . . 14

1.9 The twistor transform, Hodge DG algebra, and variations of real MHS . . . . . . 17

1.10 Mixed $\mathbb{R}$-Hodge structure on $\pi_{1}^{\text {nil }}$ via Hodge correlators . . . . . . . . . . . . . 19

1.11 Motivic correlators on curves . . . . . . . . . . . . . . . . . . . 19

1.12 Motivic multiple $L$-values . . . . . . . . . . . . . . . . . . . . . . . . . . . . . . . . . . .

1.13 Coda: Feynman integrals and motivic correlators . . . . . . . . . . . . 25

1.14 The structure of the paper . . . . . . . . . . . . . . . . 25

2 Polydifferential operator $\omega_{m} \quad \underline{27}$

2.1 The form $\omega_{m}\left(\varphi_{1}, \ldots, \varphi_{m}\right)$ and its properties $\ldots \ldots \ldots \ldots \ldots \ldots$

2.2 Twistor definition of the operator $\omega_{m} \ldots \ldots \ldots \ldots$. . . . . . . . . . . . .

3 Hodge correlators for curves $\quad 32$

3.1 Green functions on a Riemann surface . . . . . . . . . . . . . . . . . 32

3.2 Construction of Hodge correlators for curves . . . . . . . . . . . . . . . . . . . . . . . . . . . .

3.3 Shuffle relations for Hodge correlators . . . . . . . . . . . . . . 37

4 The twistor transform and the Hodge complex functor 39

4.1 The Dolbeaut complex of a variation of Hodge structures. . . . . . . . . . . . . . 39

4.2 The Hodge complex functor $\mathcal{C}_{\mathcal{H}}^{\bullet}(-) \ldots \ldots \ldots \ldots \ldots \ldots$

4.3 The twistor transform and the DGCom structure on Hodge complexes . . . . . . 44

4.4 Concluding remarks . . . . . . . . . . . . . . . . . . 52 
5 Twistor connections and variations of mixed Hodge structures 5

5.1 Green data and twistor connections . . . . . . . . . . . . . . . . . 54

5.2 Deligne's description of triples of opposite filtrations . . . . . . . . . . . . 56

5.3 Proof of Theorem $5.7 \ldots \ldots \ldots \ldots \ldots \ldots \ldots \ldots$

5.4 Conclusions . . . . . . . . . . . . . . . . . . . . . . . . . . . 62

5.5 Variations of real mixed Hodge structures. . . . . . . . . . . . . . 63

5.6 DG generalizations . . . . . . . . . . . . . . . . . . 63

6 DG Lie algebra $\mathcal{C}_{H, S}$ and $L_{\infty}$-algebra of plane decorated trees 6

6.1 A Lie algebra structure on $\mathcal{C}_{H, S} \ldots \ldots \ldots \ldots \ldots$

6.2 A commutative differential graded algebra of plane decorated forests . . . . . . 66

7 The Hodge correlator twistor connection $\quad 71$

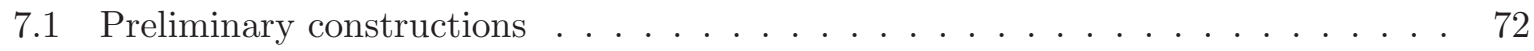

7.2 The Hodge correlator twistor connection . . . . . . . . . . . . . 73

7.3 Proof of Theorem $7.8 \ldots \ldots \ldots \ldots \ldots$

8 The Lie algebra of special derivations 8

8.1 The linear algebra set-up . . . . . . . . . . . . . . . . . . . . . . . . . . .

8.2 Special derivations of the algebra $\bar{A}_{H, S} \ldots \ldots \ldots \ldots \ldots$

8.3 A symmetric description of the Lie algebra of special derivations . . . . . . 85

9 Variations of mixed $\mathbb{R}$-Hodge structures via Hodge correlators 8

10 Motivic correlators on curves

10.1 The motivic framework . . . . . . . . . . . . . . . . . . . . . . . . . . . .

$10.2 \mathcal{C}$-motivic fundamental algebras of curves . . . . . . . . . . . . . . . . . . . . . . . . . . .

10.3 The Lie algebra of special derivations in the motivic set-up . . . . . . . . . . . 94

10.4 Motivic correlators on curves . . . . . . . . . . . . . . . . . 96

10.5 Examples of motivic correlators . . . . . . . . . . . . . . . . 99

11 Examples of Hodge correlators $\quad 102$

11.1 Hodge correlators on $\mathbb{C P}^{1}-S$ and polylogarithms $\ldots \ldots \ldots . \ldots . \ldots 102$

11.2 Hodge correlators on elliptic curves are multiple Eisenstein-Kronecker series . . . 110

12 Motivic correlators on modular curves $\mathbf{1 1 5}$

12.1 Hodge correlators on modular curves and generalized Rankin-Selberg integrals . 115

12.2 Motivic correlators in towers . . . . . . . . . . . . . . . 118

12.3 Motivic correlators for the universal modular curve . . . . . . . . . . . . . 121

13 Feynman integral for the Hodge correlators 124

13.1 The Feynman integral . . . . . . . . . . . . . . . . . . . . 124

13.2 Feynman rules . . . . . . . . . . . . . . . . . . . . . . 125

13.3 Hodge correlators and the matrix model . . . . . . . . . . . 128

Abstract 
Hodge correlators are complex numbers given by certain integrals assigned to a smooth complex curve. We show that they are correlators of a Feynman integral, and describe the real mixed Hodge structure on the pronilpotent completion of the fundamental group of the curve. We introduce motivic correlators, which are elements of the motivic Lie algebra and whose periods are the Hodge correlators. They describe the motivic fundamental group of the curve. We describe variations of real mixed Hodge structures on a variety by certain connections on the product of the variety by an affine line. We call them twistor connections. Generalising this, we suggest a DG enhancement of the subcategory of Saito's Hodge complexes with smooth cohomology. We show that when the curve varies, the Hodge correlators are the coefficients of the twistor connection describing the corresponding variation of real MHS. Examples of the Hodge correlators include classical and elliptic polylogarithms, and their generalizations. The simplest Hodge correlators on the modular curves are the Rankin-Selberg integrals. Examples of the motivic correlators include Beilinson's elements in the motivic cohomology, e.g. the ones delivering the Beilinson - Kato Euler system on modular curves.

\section{Introduction}

\section{$1.1 \quad$ Summary}

Let $X$ be a smooth compact complex curve, $v_{0}$ a non-zero tangent vector at a point $s_{0}$ of $X$, and $S^{*}=\left\{s_{1}, \ldots, s_{m}\right\}$ a collection of distinct points of $X$ different from $s_{0}$. We introduce Hodge correlators related to this datum. They are complex numbers, given by integrals of certain differential forms over products of copies of $X$. Let $S=S^{*} \cup\left\{s_{0}\right\}$. When the data $\left(X, S, v_{0}\right)$ varies, Hodge correlators satisfy a system of non-linear quadratic differential equations. Using this, we show that Hodge correlators encode a variation of real mixed Hodge structures, called below mixed $\mathbb{R}$-Hodge structures. We prove that it coincides with the standard mixed $\mathbb{R}$-Hodge structure on the pronilpotent completion $\pi_{1}^{\text {nil }}\left(X-S, v_{0}\right)$ of the fundamental group of $X-S$. The latter was defined, by different methods, by J. Morgan [M], R. Hain [ $\underline{\mathrm{H}}]$ and A.A. Beilinson (cf. [DG]). The real periods of $\pi_{1}^{\text {nil }}\left(X-S, v_{0}\right)$ have a well known description via Chen's iterated integrals. Hodge correlators give a completely different way to describe them.

Mixed Hodge structures are relatives of $l$-adic Galois representations. There are two equivalent definitions of mixed $\mathbb{R}$-Hodge structures: as vector spaces with the weight and Hodge filtrations satisfying some conditions [D], and as representations of the Hodge Galois group [D2].

The true analogs of $l$-adic representations are Hodge Galois group modules. However usually we describe mixed $\mathbb{R}$-Hodge structures arising from geometry by constructing the weight and Hodge filtrations, getting the Hodge Galois group modules only a posteriori. The Hodge correlators describe the mixed $\mathbb{R}$-Hodge structure on $\pi_{1}^{\text {nil }}\left(X-S, v_{0}\right)$ directly as a module over the Hodge Galois group.

We introduce a Feynman integral related to $X$. It does not have a rigorous mathematical meaning. However the standard perturbative series expansion procedure provides a collection of its correlators assigned to the data $\left(X, S, v_{0}\right)$, which turned out to be convergent finite dimensional integrals. We show that they coincide with the Hodge correlators, thus explaining the name of the latter. Moreover:

We define motivic correlators. Their periods are the Hodge correlators. Motivic correlators lie in the motivic Lie coalgebra, and describe the motivic fundamental group of $X-S$. The coproduct in the motivic Lie coalgebra is a new feature, which is missing when we work just 
The real mixed Hodge structure on the pronilpotent completion of the fundamental group of $\mathrm{X}-\mathrm{S}$

$$
\text { Hodge } \uparrow \text { realization }
$$

The motivic fundamental group of $\mathrm{X}-\mathrm{S}$

\author{
Hodge correlators - \\ correlators of a Feynman integral \\ related to $(\mathrm{X}, \mathrm{S})$ \\ Real $\uparrow$ periods
}

Motivic correlators live in the motivic Lie coalgebr:

with numbers. We derive a simple explicit formula for the coproduct of motivic correlators. It allows us to perform arithmetic analysis of Hodge correlators. This is one of the essential advantages of the Hodge correlator description of the real periods of $\pi_{1}^{\text {nil }}(X-S)$, which has a lot of arithmetic applications. It was available before only for the rational curve case [G7].

The Lie algebra of the unipotent part of the Hodge Galois group is a free graded Lie algebra. Choosing a set of its generators we arrive at a collection of periods of a real MHS. We introduce new generators of the Hodge Galois group, which differ from Deligne's generators [D2]. The periods of variations mixed $\mathbb{R}$-Hodge structures corresponding to these generators satisfy nonlinear quadratic Maurer-Cartan type differential equations. For the subcategory of Hodge-Tate structures they were defined by A. Levin $[\mathrm{L}$. The Hodge correlators are the periods for this set of generators.

We introduce a DG Lie coalgebra $\mathcal{L}_{\mathcal{H}, X}^{*}$. The category of $\mathcal{L}_{\mathcal{H}, X^{-}}^{*}$-comodules is supposed to be a DG-enhancement of the category of smooth $\mathbb{R}$-Hodge sheaves, i.e. the subcategory of Saito's mixed $\mathbb{R}$-Hodge sheaves whose cohomology are variations of mixed $\mathbb{R}$-Hodge structures. We show that the category of comodules over the Lie coalgebra $H^{0} \mathcal{L}_{\mathcal{H}, X}^{*}$ is equivalent to the category of variations of mixed $\mathbb{R}$-Hodge structures.

The simplest Hodge correlators for the rational and elliptic curves deliver single-valued versions of the classical polylogarithms and their elliptic counterparts, the classical EisensteinKronecker series $[\mathrm{We}$. The latter were interpreted by A.A. Beilinson and A. Levin $[\mathrm{BL}$ as periods of variations of mixed $\mathbb{R}$-Hodge structures. More generally, when $X=\mathbb{C P}^{1}$ the Hodge correlators are real periods of variations of mixed Hodge structures related to multiple polylogarithms. For an elliptic curve $E$ they deliver the multiple Eisenstein-Kronecker series defined in G1].

When $X$ is a modular curve and $S$ is the set of its cusps, Hodge correlators generalize the Rankin-Selberg integrals. Indeed, one of the simplest of them is the Rankin-Selberg convolution of a pair $f_{1}, f_{2}$ of weight two cuspidal Hecke eigenforms with an Eisenstein series. It computes, up to certain constants, the special value $L\left(f_{1} \times f_{2}, 2\right)$. A similar Hodge correlator gives the RankinSelberg convolution of a weight two cuspidal Hecke eigenform $f$ and two Eisenstein series, and computes, up to certain constants, $L(f, 2)$. (A generalization to higher weight modular forms will appear elsewhere).

The simplest motivic correlators on modular curves deliver Beilinson's elements in motivic cohomology, e.g. the Beilinson-Kato Euler system in $K_{2}$. We use motivic correlators to define motivic multiple L-values related to: Dirichlet characters of $\mathbb{Q}$ (for $X=\mathbb{C}^{*}-\mu_{N}$, where $\mu_{N}$ is the group of $N$-th roots of unity); Hecke Grössencharacters imaginary quadratic fields (for CM elliptic curves minus torsion points); Jacobi Grössencharacters of cyclotomic fields (for affine Fermat curves); the weight two modular forms. 
The Hodge correlators considered in this paper admit a generalization when $X-S$ is replaced by an arbitrary regular complex projective variety. We prove that they describe the mixed $\mathbb{R}$ Hodge structure on the rational motivic homotopy type of $X$, defined by a different method in [M]. We describe them in a separate paper since the case of curves is more transparent, and so far has more application: 1 .

Key constructions of this paper were outlined in Sections 8-9 of [G1], which may serve as an introduction.

\subsection{Arithmetic motivation I: special values of $L$-functions}

Beilinson's conjectures [B] imply that special values of $L$-functions of motives over $\mathbb{Q}$ at the integral points to the left of the critical line are periods of mixed motives over $\mathbb{Q}$. This means that they can be written as integrals

$$
\int_{\Delta_{B}} \omega_{A}
$$

where $A$ and $B$ are normal crossing divisors over $\mathbb{Q}$ in a smooth $n$-dimensional projective variety $X$ over $\mathbb{Q}, \omega_{A} \in \Omega_{\log }^{n}(X-A)$ is an $n$-form with logarithmic singularities at $A$, and $\Delta_{B}$ is an $n$-chain with the boundary on $B(\mathbb{C})$. Moreover, the special values are periods of their motivic avatars - the motivic $\zeta$-elements - which play a crucial role in arithmetic applications.

The classical example is given by the Leibniz formula for the special values of the Riemann $\zeta$-function:

$$
\zeta(n)=\int_{0 \leq t_{1} \leq \ldots \leq t_{n} \leq 1} \frac{d t_{1}}{1-t_{1}} \wedge \frac{d t_{2}}{t_{2}} \cdot \wedge \ldots \wedge \frac{d t_{n}}{t_{n}} .
$$

The corresponding motivic $\zeta$-elements were extensively studied ([BK], $[\mathrm{BD},[\mathrm{HW}])$. Beilinson's conjectures [B] are known for the special values of the Dedekind $\zeta$-function of a number field thanks to the work of Borel [Bo1], Bo2].

Here is another crucial example. Let $f(z)$ be a weight two cuspidal Hecke eigenform. So $f(z) d z$ is a holomorphic 1 -form on a compactified modular curve $\bar{M}$. Recall that by the ManinDrinfeld theorem the image in the Jacobian of any degree zero cuspidal divisor $a$ on $\bar{M}$ is torsion. So there is a modular unit $g_{a} \in \mathcal{O}^{*}(M)$ such that $\operatorname{div}\left(g_{a}\right)$ is a multiple of the divisor $a$. According to Bloch and Beilinson [B] we get an element

$$
\left\{g_{a}, g_{b}\right\} \in K_{2}(\bar{M}) \otimes \mathbb{Q} .
$$

Applying the regulator map to this element, and evaluating it on the 1-form $f(z) d z$, we get an integral

$$
\int_{\bar{M}(\mathbb{C})} \log \left|g_{a}\right| \bar{\partial} \log \left|g_{b}\right| f(z) d z
$$

It is a Rankin-Selberg convolution integral. Indeed, $\log \left|g_{a}\right|$ is the value of a non-holomorphic Eisenstein series at $s=1$, and $\partial \log \left|g_{b}\right|$ is a holomorphic weight two Eisenstein series. Therefore according to the Rankin - Selberg method, the integral is proportional to the product of special

\footnotetext{
${ }^{1}$ The case of a smooth compact Kahler manifold was treated in GII
} 
values of $L(f, s)$ at $s=1$ and $s=2$. Moreover (see [SS] for a detailed account) one can find cuspidal divisors $a$ and $b$ such that the proportionality coefficient is non-zero, i.e.

$$
\int_{\bar{M}(\mathbb{C})} \log \left|g_{a}\right| \bar{\partial} \log \left|g_{b}\right| f(z) d z \sim L(f, 2) .
$$

Using the functional equation for $L(f, s)$, one can easily deduce that $L^{\prime}(f, 0)$ is a period. Finally, suitable modifications of Beilinson's $\zeta$-elements (11) give rise to Kato's Euler system [Ka.

There are similar results, due to Beilinson [B2] in the weight two case, and Beilinson (unpublished) and, independently, Scholl (cf. [DS]) for the special values of L-functions of cuspidal Hecke eigenforms of arbitrary weight $w \geq 2$ at any integral point to the left of the critical strip, that is at $s \in \mathbb{Z}_{\leq 0}$.

This picture, especially the motivic $\zeta$-elements, seem to come out of the blue. Furthermore, already for $L^{\prime \prime}\left(\operatorname{Sym}^{2} f, 0\right)$, related by the functional equation to $L\left(\operatorname{Sym}^{2} f, 3\right)$ we do not know in general how to prove that it is a period.

One may ask whether there is a general framework, which delivers naturally both RankinSelberg integrals (2) and Beilinson's $\zeta$-elements (1), and tells where to look for generalizations related to non-critical special values $L\left(\operatorname{Sym}^{m} f, n\right)$.

We suggest that one should look at the motivic fundamental group of the universal modular curve. We show that the simplest Hodge correlator for the modular curve coincides with the Rankin-Selberg integral (2), and the corresponding motivic correlator is Beilinson's motivic $\zeta$-element (10) - see Section 1.11 for an elaborate discussion.

We will show elsewhere that the Rankin-Selberg integrals related to the values at non-critical special points of $L$-functions of arbitrary cuspidal Hecke eigenforms and the corresponding motivic $\zeta$-elements appear naturally as the Hodge and motivic correlators related to the standard local systems on the modular curve: the present paper deals with the trivial local system.

\subsection{Arithmetic motivation II: arithmetic analysis of periods}

The goal of arithmetic analysis is to investigate periods without actually computing them, using instead arithmetic theory of mixed motives 2 Here is the simplest non-trivial example, provided by the values of the dilogarithm at rational numbers. Recall that the classical dilogarithm

$$
\mathrm{Li}_{2}(z):=-\int_{0}^{z} \log (1-t) \frac{d t}{t}
$$

is a multivalued function on $\mathbb{C P}^{1}-\{0,1, \infty\}$. Its value at $z$ depends on the homotopy class of the path from 0 to $z$ on $\mathbb{C P}^{1}-\{0,1, \infty\}$ used to define the integral. Computing the monodromy of the dilogarithm, we see that $\operatorname{Li}_{2}(z)$ is well defined modulo the subgroup of $\mathbb{C}$ generated by $(2 \pi i)^{2}$ and $2 \pi i \log (z)$. So the values $\operatorname{Li}_{2}(z)$ at $z \in \mathbb{Q}$ are well defined modulo the subgroup $2 \pi i \log \mathbb{Q}^{*}$ spanned by $\mathbb{Z}(2):=(2 \pi i)^{2} \mathbb{Z}$ and $2 \pi i \log q, q \in \mathbb{Q}^{*}$ - the latter is well defined modulo $\mathbb{Z}(2)$.

Let $\mathbb{Q}^{*}$ denotes the multiplicative group of the field $\mathbb{Q}$, and $\mathbb{Q}^{*} \otimes \mathbb{Q}^{*}$ the tensor product of two abelian groups $\mathbb{Q}^{*}$. Consider a map of abelian groups

$$
\Delta: \mathbb{Z}\left[\mathbb{Q}^{*}-\{1\}\right] \longrightarrow \mathbb{Q}^{*} \otimes \mathbb{Q}^{*}, \quad\{z\} \longmapsto-(1-z) \otimes z .
$$

\footnotetext{
${ }^{2}$ The latter so far mostly is a conjectural theory, so we often arrive to conjectures rather then theorems.
} 
Conjecture 1.1. Let $z_{i} \in \mathbb{Q}, a_{j}, b_{j} \in \mathbb{Q}^{*}$ and $n_{i} \in \mathbb{Z}$. Then one has

$$
\sum_{i} n_{i} \operatorname{Li}_{2}\left(z_{i}\right)+\sum_{j} \log a_{j} \cdot \log b_{j}=0 \bmod \left(2 \pi i \log \mathbb{Q}^{*}\right)
$$

if and only if

$$
\Delta\left(\sum_{i} n_{i}\left\{z_{i}\right\}\right)+\sum_{j}\left(a_{j} \otimes b_{j}+b_{j} \otimes a_{j}\right)=0 \text { in }\left(\mathbb{Q}^{*} \otimes \mathbb{Q}^{*}\right) \otimes_{\mathbb{Z}} \mathbb{Q} .
$$

This gives a complete conjectural description of the $\mathbb{Q}$-linear relations between the values of the dilogarithm and the products of two logarithms at rational numbers.

Theorem 1.2. The condition (6) implies (5).

This tells the identities between integrals without actually computing the integrals.

Example 1.3. One has

$$
\mathrm{Li}_{2}\left(\frac{1}{3}\right)+\mathrm{Li}_{2}\left(-\frac{1}{2}\right)+\frac{1}{2}\left(\log \frac{3}{2}\right)^{2}=0 \bmod \left(2 \pi i \log \mathbb{Q}^{*}\right) .
$$

Indeed, modulo 2 -torsion in $\mathbb{Q}^{*} \otimes \mathbb{Q}^{*}$ (notice that $3 / 2 \otimes(-1)$ is a 2 -torsion) one has:

$$
\Delta\left(\left\{\frac{1}{3}\right\}+\left\{-\frac{1}{2}\right\}\right)=-\left(1-\frac{1}{3}\right) \otimes \frac{1}{3}-\left(1+\frac{1}{2}\right) \otimes\left(-\frac{1}{2}\right)=-\frac{2}{3} \otimes \frac{1}{3}-\frac{3}{2} \otimes \frac{1}{2}=-\frac{3}{2} \otimes \frac{3}{2} .
$$

Motivic avatars of the logarithm and the dilogarithm. The values of the dilogarithm and the product of two logarithms at rational arguments are weight two mixed Tate periods over $\mathbb{Q}$. In fact they describe all such periods. Here is the precise meaning of this.

The category of mixed Tate motives over a number field $F$ is canonically equivalent to the category of graded comodules over a Lie coalgebra $\mathcal{L}_{\bullet}(F)$ over $\mathbb{Q}$, graded by positive integers, the weights (cf. [DG]). The graded Lie coalgebra $\mathcal{L} \bullet(F)$ is free, with the space of generators in degree $n$ isomorphic to $K_{2 n-1}(F) \otimes \mathbb{Q}$. Equivalently, the kernel of the coproduct map

$$
\delta: \mathcal{L}_{\bullet}(F) \longrightarrow \Lambda^{2} \mathcal{L}_{\bullet}(F)
$$

is isomorphic to the graded space $\oplus_{n>0} K_{2 n-1}(F) \otimes \mathbb{Q}$, where $K_{2 n-1}(F) \otimes \mathbb{Q}$ is in degree $n$. One has $K_{1}(F)=F^{*}$, and the group $K_{2 n-1}(F)$ is of finite rank, which is $r_{1}+r_{2}$ for $n$ odd and $r_{2}$ for $n$ even due to result of Borel Bo2]. Here $r_{1}$ and $r_{2}$ are the numbers of real and complex places of $F$, so that $[F: \mathbb{Q}]=2 r_{2}+r_{1}$. It follows that $\mathcal{L}_{1}(F)=F^{*} \otimes_{\mathbb{Z}} \mathbb{Q}$. Let us describe the $\mathbb{Q}$-vector space $\mathcal{L}_{2}(F)$.

For any field $K$, consider a version of the map (4):

$$
\delta: \mathbb{Z}\left[K^{*}-\{1\}\right] \longrightarrow K^{*} \wedge K^{*}, \quad\{z\} \longmapsto-(1-z) \wedge z .
$$

Let $r(*, *, *, *)$ be the cross-ratio of four points on $\mathbb{P}^{1}$, normalized by $r(\infty, 0,1, x)=x$. Let $R_{2}(K)$ be the subgroup of $\mathbb{Z}\left[K^{*}-\{1\}\right]$ generated by the elements

$$
\sum_{i=1}^{5}(-1)^{i}\left\{r\left(x_{1}, \ldots, \widehat{x}_{i}, \ldots, x_{5}\right)\right\}, \quad x_{i} \in P^{1}(F), \quad x_{i} \neq x_{j} .
$$

Then $\delta\left(R_{2}(K)\right)=0$. Let $B_{2}(K):=\mathbb{Z}\left[K^{*}-\{1\}\right] / R_{2}(K)$ be the Bloch group of $K$. The following proposition is deduced in Section 1 of [G10] from a theorem of Suslin [S]. 
Proposition 1.4. Let $F$ be a number field. Then one has $\mathcal{L}_{2}(F)=B_{2}(F) \otimes_{\mathbb{Z}} \mathbb{Q}$. The same should be true for any field $F$. However the Lie coalgebra $\mathcal{L} \bullet(F)$ in that case is a conjectural object.

The dual of the universal enveloping algebra of the Lie algebra graded dual to the Lie coalgebra $\mathcal{L}_{\bullet}(F)$ is a commutative graded Hopf algebra 3 Its weight two component is

$$
\mathcal{L}_{2}(F) \oplus \operatorname{Sym}^{2} \mathcal{L}_{1}(F)=\left(B_{2}(F) \oplus \operatorname{Sym}^{2} F^{*}\right) \otimes_{\mathbb{Z}} \mathbb{Q} .
$$

The coproduct is provided by the map

$$
\begin{gathered}
\Delta_{2}: B_{2}(F) \oplus \operatorname{Sym}^{2} F^{*} \longrightarrow\left(F^{*} \otimes F^{*}\right) \otimes_{\mathbb{Z}} \mathbb{Q} \\
\{z\}_{2} \longmapsto \frac{1}{2}(z \otimes(1-z)-(1-z) \otimes z), \quad a \cdot b \longmapsto a \otimes b+b \otimes a .
\end{gathered}
$$

Let us return to our example, when $F=\mathbb{Q}$. The period homomorphism

$$
\mathcal{L}_{1}(\mathbb{Q}) \longrightarrow \mathbb{C} / \mathbb{Z}(1), \quad q \longmapsto \log q
$$

is known to be injective. So $q \in \mathbb{Q}^{*}$ is the motivic avatar of $\log q$.

Proposition 1.5. There is a weight two period map

$$
\begin{gathered}
\mathcal{L}_{2}(\mathbb{Q}) \oplus \operatorname{Sym}^{2} \mathcal{L}_{1}(\mathbb{Q}) \longrightarrow \mathbb{C} / 2 \pi i \log \mathbb{Q}^{*}, \\
\{q\}_{2} \longmapsto \operatorname{Li}_{2}(q)+\frac{1}{2} \log (1-q) \log (q), \quad a \otimes b+b \otimes a \longmapsto \log a \log b .
\end{gathered}
$$

The point is that the period map kills the subgroup $R_{2}(\mathbb{C})$, which is equivalent to Abel's five term relation for the dilogarithm.

The period map (12) is conjectured to be injective: this is a special case of a Grothendieck type conjecture on periods. Finally, $\operatorname{Ker} \Delta_{2}=K_{3}(\mathbb{Q}) \otimes \mathbb{Q}$, which is zero by Borel's theorem. The injectivity of $\Delta_{2}$ plus Propositions 1.4 and 1.5 imply Theorem 1.2, and, modulo the injectivity of the period map (12), Conjecture 1.1.

Although relation (17) follows from the five term relation for the dilogarithm, there is no algorithm which allows to write the element $\left\{\frac{1}{3}\right\}+\left\{-\frac{1}{2}\right\}$ as a sum of the five term relations (9). The only effective way to know that they exist is to calculate the coproduct, as we did in (8).

Let us now discuss the case when $F$ is an arbitrary number field.

Real periods. There is a single valued version of the dilogarithm, the Bloch-Wigner function

$$
\mathcal{L}_{2}(z):=-\operatorname{Im}\left(\int_{0}^{z} \log (1-t) \frac{d t}{t}+\int_{0}^{z} \frac{d t}{1-t} \cdot \log |z|\right) .
$$

where both integrals are defined by using the same integration path from 0 to $z$. It satisfies the five term relation, and thus provides a group homomorphism

$$
B_{2}(\mathbb{C}) \longrightarrow \mathbb{R}, \quad\{z\}_{2} \rightarrow \mathcal{L}_{2}(z) .
$$

\footnotetext{
${ }^{3}$ We want to note that although it is traditionally graded by the nonnegative integers, the Tate weights, there is no sign rule involved here. Perhaps we would be better off by multiplyinbg the weights by the factor of two.
} 
Using the (conjectural) isomorphism $\mathcal{L}_{2}(\mathbb{C})=B_{2}(\mathbb{C})$, it can be interpreted as the real period map $\mathcal{L}_{2}(\mathbb{C}) \rightarrow \mathbb{R}$. Notice that $\mathcal{L}_{2}(z)=0$ for $z \in \mathbb{R}$. So the real period map looses a lot of information about the motivic Lie algebra. It relates, however, the kernel of the coproduct map with the special values of $L$-functions. Here is how it works in our running example.

Let $F$ be a number field. Then by Borel's theorem Bo2 the real period map provides an injective regulator map

$$
K_{3}(F) \otimes \mathbb{Q} \cong \operatorname{Ker} \delta \subset B_{2}(F) \otimes \mathbb{Q} \longrightarrow \mathbb{R}^{r_{2}}, \quad\{z\}_{2} \longmapsto\left(\mathcal{L}_{2}\left(\sigma_{1}(z)\right), \ldots, \mathcal{L}_{2}\left(\sigma_{r_{2}}(z)\right)\right) .
$$

Its image is a rational lattice - that is, a lattice tensor $\mathbb{Q}-$ in $\mathbb{R}^{r_{2}}$, whose covolume, well defined up to $\mathbb{Q}^{*}$, is a $\mathbb{Q}^{*}$-multiple of $\zeta_{F}(-1)$.

So to perform the arithmetic analysis of the values of the dilogarithm / products of two logarithms at rational arguments we upgrade them to their motivic avatars lying in (10), and determine their coproduct there. The kernel of the coproduct is captured by the regulator map.

The weight $m$ periods of mixed Tate motives over a number field $F$ are studied similarly using the iterated coproduct in $\otimes^{m} F^{*}$, see Section 4 of [G7].

In this paper we develope a similar picture for the periods of the pronilpotent completions of fundamental groups of curves. Namely, we introduce motivic correlators, which are the motivic avatars of the periods of fundamental groups of curves. They span a Lie subcoalgebra in the motivic Lie coalgebra. The key point is that the coproduct of a motivic correlator is given explicitly in terms of the motivic correlators. We prove that their real periods are given by the Hodge correlators.

\subsection{Mixed motives and the motivic Lie algebra}

Let us recall the conjectural motivic setting. For any field $F$ there is a conjectural abelian category of pure Grothendieck motives over $F$. It is expected to be a semisimple abelian tensor category. Moreover, there is a conjectural abelian category of mixed motivic sheaves over any base $X$, whose properties were discussed by Beilinson in [B1]. In particular it contains the Tate motives over $X$, denoted by $\mathbb{Q}(n)_{X}$. Let us asume that $X$ is regular. The Ext groups between them in this category are related by Beilinson's formula [B1] to the Adams graded quotients of the algebraic K-groups of the base $X$ :

$$
\operatorname{Ext}^{m}\left(\mathbb{Q}(0)_{X}, \mathbb{Q}(n)_{X}\right)=\operatorname{gr}_{\gamma}^{n} K_{2 n-m}(X) \otimes \mathbb{Q} .
$$

A useful tool investigate the Ext groups in general is the hypothetical motivic Leray spectral sequence. Let us assume that $X$ is a regular projective variety over a field $F$. Then using (13) and the pair of adjoint functors $p^{*}, p_{*}$ where $p: X \rightarrow \operatorname{Spec}(F)$, we have

$\operatorname{gr}_{\gamma}^{n} K_{2 n-m}(X) \otimes \mathbb{Q}=\operatorname{Ext}^{m}\left(\mathbb{Q}(0)_{X}, \mathbb{Q}(n)_{X}\right)=\operatorname{Ext}^{m}\left(p^{*} \mathbb{Q}(0)_{F}, \mathbb{Q}(n)_{X}\right)=\operatorname{Ext}^{m}\left(\mathbb{Q}(0)_{F}, p_{*} \mathbb{Q}(n)_{X}\right)$

Since $p_{*} \mathbb{Q}(n)_{X}$ is expected to have a filtration with the associate graded are pure motives $h^{i} X(n)$, we expect a spectral sequence with the $E^{2}$-term

$$
\operatorname{Ext}^{j}\left(\mathbb{Q}(0)_{F}, h^{i} X(n)\right)=>\operatorname{gr}_{\gamma}^{n} K_{2 n-m}(X) \otimes \mathbb{Q}
$$

The trianguilated category of motives is available, together with Beilinson's formula (13), thatnks to the works of V. Voevodsky $[\mathrm{V}]$ and M. Levine [Le]. The abelian category of mixed motives 
is not available yet. However if we restrict to the case when $X=\operatorname{Spec}\left(\mathcal{O}_{F, S}\right)$ is the spectrum of the ring of $S$-integers in a number field $F$, and consider the subcategory of mixed Tate motives, the corresponding category with all necessary properties is available [Le2] for $X=\operatorname{Spec}(F)$ and DG.

Let us assume that $X=\operatorname{Spec}(F)$ where $F$ is a field. The Tannakian category of mixed motives over $F$ is expected to be canonically equivalent to the category of modules over a Lie algebra $\mathrm{L}_{\mathrm{Mot} / \mathrm{F}}$ in the category of pure motives over $\operatorname{Spec}(F)$. We also use notation $\mathrm{L}_{\mathrm{Mot}}$. The equivalence is provided by the fiber functor $M \longmapsto \operatorname{gr}^{W} M$. Denote by $\mathcal{L}_{\text {Mot } / \mathrm{F}}$ the dual Lie coalgebra. So there is an isomorphism

$$
H^{i}\left(\mathcal{L}_{\mathrm{Mot} / \mathrm{F}}\right)=\oplus_{[M]} \operatorname{Ext}_{\mathrm{Mot} / \mathrm{F}}^{i}(\mathbb{Q}(0), M) \otimes M^{\vee} .
$$

where the sum is over the isomorphism classes of pure motives. In particular, one has

$$
\operatorname{Ker}\left(\mathcal{L}_{\mathrm{Mot} / \mathrm{F}} \stackrel{\delta}{\longrightarrow} \Lambda^{2} \mathcal{L}_{\mathrm{Mot} / \mathrm{F}}\right)=\oplus_{[M]} \operatorname{Ext}_{\mathrm{Mot} / \mathrm{F}}^{1}(\mathbb{Q}(0), M) \otimes M^{\vee} .
$$

If $F$ is a number field, then, conjecturally, $H^{i}\left(\mathcal{L}_{\mathrm{Mot} / \mathrm{F}}\right)=0$ for $i>1$. So the motivic Lie algebra $L_{\mathrm{Mot} / \mathrm{F}}$ is a free Lie algebra in the category of pure motives. So (17) implies that the $M^{\vee}$-isotipical component of the space of generators is isomorphic to $\operatorname{Ext}_{\text {Mot } / \mathrm{F}}^{1}(\mathbb{Q}(0), M)$.

Finally, the size of $\operatorname{Ext}_{\text {Mot } / \mathrm{F}}^{1}(\mathbb{Q}(0), M)$ is predicted by Beilinson's conjecture $[\mathrm{B}]$ on regulators.

We conclude that in the case when $F$ is a number field, the structure of the motivic Lie algebra $\mathrm{L}_{\mathrm{Mot} / \mathrm{F}}$ is completely predicted by Beilinson's conjectures.

\subsection{Pronilpotent completions of fundamental groups of curves}

The fundamental group $\pi_{1}=\pi_{1}\left(X-S, v_{0}\right)$ is a free group with generators provided by loops around the rest of the punctures $s_{i} \neq s_{0}$ and loops generating $H_{1}(X)$. Let $\mathcal{I}:=\operatorname{Ker}(\mathbb{Q}[\pi] \rightarrow \mathbb{Q})$ be the augmentation ideal of the group algebra of $\pi_{1}$. Then there is a complete cocommutative Hopf algebra over $\mathbb{Q}$

$$
\mathrm{A}^{\operatorname{Betti}}\left(X-S, v_{0}\right):=\lim _{\longleftarrow} \mathbb{Q}\left[\pi_{1}\right] / \mathcal{I}^{n} .
$$

Its coproduct is induced by the map $g \longmapsto g \otimes g, g \in \pi_{1}$. It is called the fundamental Hopf algebra of $X-S$ with the tangential base point $v_{0}$. The subset of its primitive elements is a free pronilpotent Lie algebra over $\mathbb{Q}$, the Maltsev completion of $\pi_{1}$. It is denoted $\pi_{1}^{\text {nil }}\left(X-S, v_{0}\right)$ and called the fundamental Lie algebra of $X-S$. The Hopf algebra (18) is its universal enveloping algebra.

Denote by $\mathrm{T}(V)$ the tensor algebra of a vector space $V$. The associated graded of the Hopf algebra (18) for the $\mathcal{I}$-adic filtration is isomorphic to the tensor algebra of $H_{1}(X-S, \mathbb{Q})$ :

$$
\operatorname{gr}^{\mathcal{I}} \mathrm{A}^{\operatorname{Betti}}\left(X-S, v_{0}\right)=\oplus_{n=0}^{\infty} \mathcal{I}^{n} / \mathcal{I}^{n+1}=\mathrm{T}\left(H_{1}(X-S, \mathbb{Q})\right) .
$$

There is a weight filtration on $H_{1}(X-S, \mathbb{Q})$, given by the extension

$$
0 \longrightarrow \mathbb{Q}(1)^{|S|-1} \longrightarrow H_{1}(X-S, \mathbb{Q}) \longrightarrow H_{1}(X, \mathbb{Q}) \longrightarrow 0,
$$

where the right arrow is provided by the embedding $X-S \hookrightarrow X$. The Hopf algebra (18) is equipped with a weight filtration $W$ compatible with the Hopf algebra structure. The associated graded for the weight filtration of the Hopf algebra (18) is isomorphic to the tensor algebra of $\mathrm{gr}^{W} H_{1}(X-S, \mathbb{Q})$ :

$$
\operatorname{gr}^{W} \mathrm{~A}^{\operatorname{Betti}}\left(X-S, v_{0}\right)=\mathrm{T}\left(\operatorname{gr}^{W} H_{1}(X-S, \mathbb{Q})\right) \text {. }
$$




\subsection{Hodge correlators}

Recall $S^{*}=S-\left\{s_{0}\right\}$. Set

$$
\mathrm{V}_{X, S^{*}}:=H_{1}(X, \mathbb{C}) \oplus \mathbb{C}\left[S^{*}\right], \quad \mathrm{A}_{X, S^{*}}:=\mathrm{T}\left(\mathrm{V}_{X, S^{*}}\right) .
$$

One sees from (19), (20) that there are canonical isomorphisms of vector spaces

$$
\mathrm{V}_{X, S^{*}}=\operatorname{gr}^{W} H_{1}(X-S, \mathbb{C}), \quad \mathrm{A}_{X, S^{*}}=\operatorname{gr}^{W} \mathrm{~A}^{\operatorname{Betti}}\left(X-S, v_{0}\right) \otimes \mathbb{C} .
$$

We also need the dual objects:

$$
\mathrm{V}_{X, S^{*}}^{\vee}:=H^{1}(X, \mathbb{C}) \oplus \mathbb{C}\left[S^{*}\right], \quad \mathrm{A}_{X, S^{*}}^{\vee}:=\mathrm{T}\left(\mathrm{V}_{X, S^{*}}^{\vee}\right)
$$

For an associative algebra $A$ over $k$, let $A^{+}:=\operatorname{Ker}(A \rightarrow k)$ be the augmentation ideal. Let $\left[A^{+}, A^{+}\right]$be the subspace (not the ideal) of $A^{+}$generated by commutators in $A^{+}$. The quotient space $\mathcal{C}(A):=A^{+} /\left[A^{+}, A^{+}\right]$is called the cyclic envelope of $A$. If $A$ is freely generated by a set $\mathcal{S}$ then the vector space $\mathcal{C}(A)$ has a basis parametrised by cyclic words in $\mathcal{S}$.

We use the shorthand $\mathcal{C} \mathrm{T}(V)$ for $\mathcal{C}(\mathrm{T}(V))$. We define the subspace of shuffle relations in $\mathcal{C} \mathrm{T}(\mathrm{V})$ as the subspace generated by the elements

$$
\sum_{\sigma \in \Sigma_{p, q}}\left(v_{0} \otimes v_{\sigma(1)} \otimes \ldots \otimes v_{\sigma(p+q)}\right), \quad p, q \geq 1
$$

where the sum is over all $(p, q)$-shuffles. Set

$$
\begin{gathered}
\mathcal{C}_{X, S^{*}}^{\vee}:=\mathcal{C}\left(\mathrm{A}_{X, S^{*}}^{\vee}\right), \quad \mathcal{C} \mathcal{L} i e_{X, S^{*}}^{\vee}:=\frac{\mathcal{C}_{X, S^{*}}^{\vee}}{\text { Shuffle relations }} \\
\mathcal{C}_{X, S^{*}}:=\mathcal{C}\left(\mathrm{A}_{X, S^{*}}\right) \quad \mathcal{C} \mathcal{L} i e_{X, S^{*}}:=\text { the dual of } \mathcal{C} \mathcal{L} i e_{X, S^{*}}^{\vee} .
\end{gathered}
$$

We show that $\mathcal{C}_{X, S^{*}}^{\vee}$ is a Lie coalgebra, $\mathcal{C} \mathcal{L} i e_{X, S^{*}}^{\vee}$ is its quotient Lie coalgebra. Equivalently, $\mathcal{C}_{X, S^{*}}$ is a Lie algebra, $\mathcal{C} \mathcal{L} i e_{X, S^{*}}$ is its Lie subalgebra.

Given a mass one volume form $\mu$ on $X$, our Feynman integral construction in Section 2 provides a linear map, called the Hodge correlator map

$$
\operatorname{Cor}_{\mathcal{H}, \mu}: \mathcal{C} \mathcal{L} i e_{X, S^{*}}^{\vee} \longrightarrow \mathbb{C} \text {. }
$$

Example 1.6. For a cyclic word

$$
W=\mathcal{C}\left(\left\{a_{0}\right\} \otimes\left\{a_{1}\right\} \otimes \ldots \otimes\left\{a_{n}\right\}\right), \quad a_{i} \in S^{*},
$$

the Hodge correlator is given by a sum over all plane trivalent trees $T$ whose external edges are decorated by elements $a_{0}, \ldots, a_{n}$, see Fig 1. To define the integral corresponding to such a tree $T$ we proceed as follows. The volume form $\mu$ provides a Green function $G(x, y)$ on $X^{2}$. Each edge $E$ of $T$ contributes a Green function on $X^{\text {\{vertices of } E\}}$, which we lift to a function on $X^{\{\text {vertices of } T\}}$. Further, given any $m+1$ smooth functions on a complex manifold $M$, there is a canonical linear map $\omega_{m}: \Lambda^{m+1} \mathcal{A}_{M}^{0} \rightarrow \mathcal{A}_{M}^{m}$, where $\mathcal{A}_{M}^{k}$ is the space of smooth $k$-forms on $M$ (Section 2.2). Applying it to the Green functions assigned to the edges of $T$, we get a differential 


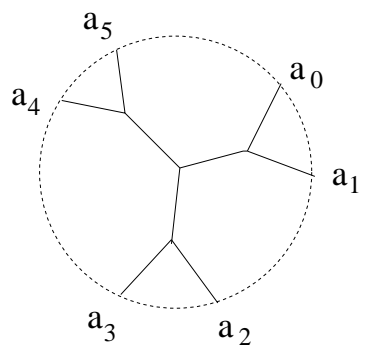

Figure 1: A plane trivalent tree decorated by $\mathcal{C}\left(\left\{a_{0}\right\} \otimes \ldots \otimes\left\{a_{5}\right\}\right)$.

form of the top degree on $X^{\text {internal vertices of } T\}}$. Integrating it, we get the integral assigned to $T$. Taking the sum over all trees $T$, we get the number $\operatorname{Cor}_{\mathcal{H}, \mu}(W)$. The shuffle relations result from taking the sum over all trees with a given decoration.

Dualising the Hodge correlator map (26), we get a Green operator

$$
\mathbf{G}_{\mu} \in \mathcal{C} \mathcal{L}_{i e_{X, S^{*}}}
$$

Let $\mathrm{L}_{X, S^{*}}$ be the free Lie algebra generated by the space $\mathrm{V}_{X, S^{*}}$. There is a canonical isomorphism

$$
\mathrm{L}_{X, S^{*}}=\operatorname{gr}^{W} \pi_{1}^{\text {nil }}\left(X-S, v_{0}\right) \otimes \mathbb{C} .
$$

The Green operator can be viewed as a derivation of the Lie algebra $\mathrm{L}_{X, S^{*}}$ as follows.

Let $\left(p_{i}, q_{i}\right)$ be a symplectic basis of $H_{1}(X)$. Denote by $X_{s}$ the generator of $\mathrm{L}_{X, S^{*}}$ assigned to $s \in S^{*}$. There is a canonical generator of $\mathrm{gr}^{W} \pi_{1}^{\text {nil }}\left(X-S, v_{0}\right)$ corresponding to a loop around $s_{0}$. Its projection to $\mathrm{L}_{X, S^{*}}$ is written as

$$
X_{s_{0}}:=-\sum_{s \in S^{*}} X_{s}+\sum\left[p_{i}, q_{i}\right]
$$

Say that a derivation of the Lie algebra $\mathrm{L}_{X, S^{*}}$ is special if it kills the generator (30) and preserves the conjugacy classes of the generators $X_{s}$, where $s \in S^{*}$.

This definition is motivated by the $l$-adic picture (Section 10,2): If $X$ is a curve over a field $F$ and $S \subset X(F)$, the Galois group $\operatorname{Gal}\left(\bar{F} / F\left(\mu_{l^{\infty}}\right)\right)$ acts by special automorphisms of $\mathrm{L}_{X, S^{*}} \otimes \mathbb{Q}_{l}$. Indeed, by the comparison theorem the latter is identified with the associate graded for the weight filtration on the Lie algebra of the pro-l-completion $\pi_{1}^{(l)}\left(X-S, v_{0}\right)$ of the fundamental group.

Denote the Lie algebra of special derivations by $\operatorname{Der}^{S} \mathrm{~L}_{X, S^{*}}$. We show that the Lie algebra $\mathcal{C} \mathcal{L}_{e_{X, S^{*}}}$ acts by special derivations on the Lie algebra $\mathrm{L}_{X, S^{*}}$. This generalizes constructions of Drinfeld [Dr1] (when $X$ is of genus zero) and Kontsevich [K] (when $S$ is empty). Namely, let $F$ be a cyclic polynomial in non-commuting variables $Y_{i}$, i.e. $F \in \mathcal{C T}(Y)$ where $Y$ is a vector space with a basis $\left\{Y_{i}\right\}$. Then there are "partial derivatives" maps

$$
\partial / \partial Y_{i}: \mathcal{C T}(Y) \longrightarrow \mathrm{T}(Y), \quad F \longmapsto \partial F / \partial Y_{i}
$$

defined by deleting one of $Y_{i}$ 's from $F$, getting as a result a non-commutative polynomial, and taking the sum over all possibilities. For instance if $F=\mathcal{C}\left(Y_{1} Y_{2} Y_{1} Y_{3}\right)$ then $\partial F / \partial Y_{1}=$ $Y_{2} Y_{1} Y_{3}+Y_{3} Y_{1} Y_{2}$. 
Let $F$ be a cyclic polynomial in non-commuting variables $X_{s}, p_{i}, q_{i}$, where $s \in S^{*}$. Then the derivation $\kappa_{F}$ assigned to $F$ acts on the generators by

$$
p_{i} \longmapsto-\frac{\partial F}{\partial q_{i}}, \quad q_{i} \longmapsto \frac{\partial F}{\partial p_{i}}, \quad X_{s} \longmapsto\left[X_{s}, \frac{\partial F}{\partial X_{s}}\right], \quad s \in S^{*} .
$$

A priori it is a derivation of the associative algebra $\mathrm{A}_{X, S^{*}}$. However if $F$ annihilates the shuffle relations, it is a derivation of the Lie algebra $\mathrm{L}_{X, S^{*}}$. We show that we get an isomorphism

$$
\mathcal{C} \mathcal{L}_{X, e^{*}} \stackrel{\sim}{\longrightarrow} \operatorname{Der}^{S} \mathrm{~L}_{X, S^{*}}, \quad F \longmapsto \kappa_{F} .
$$

Combining (28) and (31), we conclude that the Green operator can be viewed as an element

$$
\mathbf{G}_{\mu} \in \operatorname{Der}^{S} \mathrm{~L}_{X, S^{*}}
$$

We show that it encodes a mixed $\mathbb{R}$-Hodge structure using a construction from Section 1.8 .

\subsection{A Feynman integral for Hodge correlators}

Let $\varphi$ be a smooth function on a complex curve $X(\mathbb{C})$ with values in $N \times N$ complex matrices. We say that the space $\{\varphi\}$ of such functions is our space of fields. Given $N$, consider the following correlator corresponding to $W \in \mathcal{C}_{X, S}^{\vee}$, formally defined via a Feynman integral over the space of fields $\{\varphi\}$

$$
\operatorname{Cor}_{X, N, h}(W):=\int \mathcal{F}_{W}(\varphi) e^{i S(\varphi)} \mathcal{D} \varphi
$$

where

$$
S(\varphi):=\frac{1}{2 \pi i} \int_{X} \operatorname{Tr}\left(\frac{1}{2} \partial \varphi \wedge \bar{\partial} \varphi+\frac{1}{6} \hbar \cdot \varphi[\partial \varphi, \bar{\partial} \varphi]\right) .
$$

and $\mathcal{F}_{W}(\varphi)$ is a function on the space of fields, see Section 13. For example, for the cyclic word (27) we set

$$
\mathcal{F}_{W}(\varphi):=\operatorname{Tr}\left(\varphi\left(a_{0}\right) \ldots \varphi\left(a_{n}\right)\right)
$$

Formula (33) does not have a precise mathematical meaning. We understand it by postulating the perturbative series expansion with respect to a small parameter $h$, using the standard Feynman rules, and then taking the leading term in the asymptotic expansion as $N \rightarrow \infty, \hbar=N^{-1 / 2}$. This way we get a sum over a finite number of Feynman diagrams given by plane trivalent trees decorated by the factors of $W$. One needs to specify a volume form $\mu$ on $X$ to determine a measure $\mathcal{D} \varphi$ on the space of fields. Mathematically, we need $\mu$ to specify the Green function which is used to write the perturbative series expansion.

We prove in Theorem 13.4 that these Feynman intregral correlators coincide with the suitably normalised Hodge correlators.

Problem. The Hodge correlators provide only the leading term of the $N \rightarrow \infty$ asymptotics of the Feynman integral correlators. The next terms in the asymptotics are given by the correlators corresponding to higher genus ribbon graphs. They could be divergent. How to renormalise them? What is their role in the Hodge theory? 


\subsection{Variations of mixed $\mathbb{R}$-Hodge structures by twistor connections}

Real Hodge structures. A real Hodge structure is a real vector space $V$ whose complexification is equipped with a bigrading $V_{\mathbb{C}}=\oplus V^{p, q}$ such that $\bar{V}^{p, q}=V^{q, p}$. A real Hodge structure is pure if $p+q$ is a given number, called the weight. So a real Hodge structure is a direct sum of pure ones of different weights.

The category $\mathrm{HS}_{/ \mathbb{R}}$ of real Hodge structures is equivalent to the category of representations of the algebraic group $\mathbf{G}_{m \mathbb{C} / \mathbb{R}}$. The group of its real points is the group $\mathbb{C}^{*}$. The group of its complex points is the group $\mathbb{C}^{*} \times \mathbb{C}^{*}$ with the action of the complex conjugation interchanging the factors. Abusing notation, we denote it by $\mathbb{C}_{\mathbb{C} / \mathbb{R}}^{*}$

Real mixed Hodge structures. According to P. Deligne [D], a mixed $\mathbb{R}$-Hodge structure is a real vector space $V$ equipped with a weight filtration $W_{\bullet}$, and a Hodge filtration $F^{\bullet}$ of its complexification $V_{\mathbb{C}}$, satisfying the following condition. The filtration $F^{\bullet}$ and its conjugate $\bar{F}^{\bullet}$ induce on $\operatorname{gr}_{n}^{W} V$ a pure weight $n$ real Hodge structure:

$$
\operatorname{gr}^{W} V_{\mathbb{C}}=\oplus_{p+q=n} F_{(n)}^{p} \cap \bar{F}_{(n)}^{q} .
$$

Here $F_{(n)}^{\bullet}$ is the filtration on $\operatorname{gr}_{n}^{W} V_{\mathbb{C}}$ induced by $F^{\bullet}$, and similarly $\bar{F}_{(n)}^{\bullet}$.

The real Hodge Galois group. The category $M H S / \mathbb{R}$ of mixed $\mathbb{R}$-Hodge structures is a Tannakian category with a canonical fiber functor $\omega_{\text {Hod }}$ to the category $\operatorname{Vect}_{\mathbb{R}}$ of $\mathbb{R}$-vector spaces:

$$
\omega_{\mathrm{Hod}}: \mathrm{MHS}_{\mathbb{R}} \longrightarrow \operatorname{Vect}_{\mathbb{R}}
$$

It assigns to a real mixed Hodge structure $\left(V, W \bullet V, F^{\bullet} V_{\mathbb{C}}\right)$ the underlying real vector space $V$. The Hodge Galois group $G_{\text {Hod }}$ is defined as the group of automorphisms of the fiber functor respecting the tensor structure:

$$
G_{\text {Hod }}:=\text { Aut }^{\otimes} \omega_{\text {Hod }}
$$

It is a pro-algebraic group over $\mathbb{R}$. Thanks to the Tannakian formalism, the functor $\omega_{\text {Hod }}$ provides a canonicall equivalence of the tensor category of real mixed Hodge structures with the tensor category of representations of the proalgebraic group $G_{\text {Hod }}$ :

$$
\omega_{\text {Hod }}: \text { MHS } / \mathbb{R} \stackrel{\sim}{\longrightarrow} G_{\text {Hod }}-\text { modules. }
$$

There are two canonical functors

$$
\mathrm{gr}^{W}: \mathrm{MHS}_{/ \mathbb{R}} \longrightarrow \mathrm{HS}_{/ \mathbb{R}}, \quad V \longmapsto \mathrm{gr}^{W} V, \quad i: \mathrm{HS}_{/ \mathbb{R}} \hookrightarrow \mathrm{MHS}_{\mathbb{R}}, \quad V \longmapsto V, \quad \operatorname{gr}^{W} \circ i=\mathrm{Id} .
$$

Here the functor $i$ is the canonical embedding. Therefore there are two homomorphisms

$$
s: \mathbb{C}_{\mathbb{C} / \mathbb{R}}^{*} \longrightarrow G_{\text {Hod }}, \quad p: G_{\text {Hod }} \longrightarrow \mathbb{C}_{\mathbb{C} / \mathbb{R}}^{*}, \quad p \circ s=\mathrm{Id} .
$$

These homomorphisms provide the group $G_{\text {Hod }}$ with a structure of a semidirect product of a real prounipotent algebraic group $U_{\text {Hod }}:=\operatorname{Ker} p$ and $\mathbb{C}_{\mathbb{C} / \mathbb{R}}^{*}$ :

$$
0 \longrightarrow U_{\text {Hod }} \longrightarrow G_{\text {Hod }} \stackrel{p}{\longrightarrow} \mathbb{C}_{\mathbb{C} / \mathbb{R}}^{*} \longrightarrow 0 \text {. }
$$


Let $\mathrm{L}_{\text {Hod }}$ be the Lie algebra of the group $U_{\text {Hod }}$. The action of $s\left(\mathbb{C}_{\mathbb{C} / \mathbb{R}}^{*}\right)$ provides the Lie algebra $\mathrm{L}_{\text {Hod }}$ with a structure of a Lie algebra in the category of $\mathbb{R}$-Hodge structures. The category of mixed $\mathbb{R}$-Hodge structures is equivalent to the category of representations of the Lie algebra $\mathrm{L}_{\mathrm{Hod}}$ in the category of $\mathbb{R}$-Hodge structures. The Lie algebra $\mathrm{L}_{\mathrm{Hod}}$ is a free Lie algebra in the category of $\mathbb{R}$-Hodge structures. Thus the Lie algebra $\mathrm{L}_{\text {Hod }}$ is generated by the $\mathbb{R}$-Hodge structure

$$
\oplus_{[H]} \operatorname{Ext}_{\mathrm{MHS} / \mathbb{R}}^{1}(\mathbb{R}(0), H)^{\vee} \otimes H,
$$

where the sum is over the set of isomorphism classes of simple $\mathbb{R}$-Hodge structures $H$. The ones with non-zero $\mathrm{Ext}^{1}$ are parametrised by pairs of negative integers $(-p,-q)$ up to a permutation (the Hodge degrees of $H$ ), and one has $\operatorname{dim}_{\mathbb{R}} \operatorname{Ext}_{\mathrm{MHS} / \mathbb{R}}^{1}(\mathbb{R}(0), H)=1$ for those $H$.

It follows that the complex Lie algebra $\mathrm{L}_{\text {Hod }} \otimes_{\mathbb{R}} \mathbb{C}$ is generated by certain elements $n_{p, q}$, $p, q \geq 1$, of bidegree $(-p,-q)$, with the only relation $\bar{n}_{p, q}=-n_{q, p}$. Such a generators were defined by Deligne [D2. We call them Deligne's generators.

This just means that a mixed $\mathbb{R}$-Hodge structure can be described by a pair $(V, g)$, where $V$ is a real vector space whose complexification is equipped with a bigrading $V_{\mathbb{C}}=\oplus V^{p, q}$ such that $\bar{V}^{p, q}=V^{q, p}$, and $g=\sum_{p, q \geq 1} g_{p, q}$ is an imaginary operator on $V_{\mathbb{C}}$, so that $g_{p, q}$ is of bidegree $(-p,-q)$ and $\bar{g}_{p, q}=-g_{q, p}$. The operators $g_{p, q}$ are the images of Deligne's generators $n_{p, q}$ in the representation $V$. One can describe variations of real Hodge structures using the operators $g_{p, q}$ fiberwise, but the Griffiths transversality condition is encoded by so complicated nonlinear differential equations on $g_{p, q}$ that it was not even written down.

A.A. Beilinson emphasized that Deligne's generators may not be the most natural ones, and asked whether one should exist a canonical choice of the generators. For the subcategory of mixed $\mathbb{R}$-Hodge-Tate structures (i.e. when the Hodge numbers are zero unless $p=q$ ) a different set of generators $n_{p}$ was suggested by A. Levin $[\mathrm{L}$.

Let us decompose the Hodge correlator $\mathbf{G}=\sum \mathbf{G}_{p, q}$ according to the Hodge bigrading. We proved that the $\mathbf{G}_{p, q}$ are images of certain generators of the Lie algebra $\mathrm{L}_{\text {Hod }}$, thus providing the mixed $\mathbb{R}$-Hodge structure on $\pi_{1}^{\text {nil }}\left(X-S, v_{0}\right)$. We proved in Section 7 that, when the data $\left(X, S, v_{0}\right)$ varies, the $\left\{\mathbf{G}_{p, q}\right\}$ satisfy a Maurer-Cartan nonlinear quadratic differential equations.

Motivated by this, we show that a variation of mixed $\mathbb{R}$-Hodge structures can be described by a direct sum of variations of pure Hodge structures plus a Green datum $\left\{G_{p, q}, \nu\right\}$ consisting of operator-valued functions $G_{p, q}, p, q \geq 1$ and a 1-form $\nu$, satisfying a Maurer-Cartan system of quadratic nonlinear differential equations. The Green operators $G_{p, q}$ are nonlinear modifications of Deligne's operators $g_{p, q}$, providing a canonical set of generators of the Hodge Lie algebra $\mathrm{L}_{\mathrm{Hod}}$. For the Hodge-Tate structures we recover the Green operators of [L], although even in this case our treatment is somewhat different, based on the twistor transform defined in Section 6 .

Let us describe our construction. Let $\mathcal{V}$ be a variation of real Hodge structures on $X$. It is a real local system $\mathcal{V}$, whose complexification $\mathcal{V}_{\mathbb{C}}$ has a canonical decomposition

$$
\mathcal{V}_{\mathbb{C}}=\oplus_{p, q} \mathcal{V}^{p, q}
$$

Tensoring $\mathcal{V}$ with the sheaf of smooth complex functions on $X$, we get a $C^{\infty}$-bundle $\mathcal{V}_{\infty}$ with a flat connection $\mathbf{d}$ satisfying the Griffiths transversality condition. Pick a real closed 1-form

$$
g_{0,0} \in \Omega^{1} \otimes \operatorname{End}^{-1,0} \mathcal{V}_{\infty} \oplus \bar{\Omega}^{1} \otimes \operatorname{End}^{0,-1} \mathcal{V}_{\infty}, \quad \mathbf{d} g_{0,0}=0, \quad \bar{g}_{0,0}=g_{0,0},
$$


and a collection of imaginary operators

$$
G_{p, q} \in \text { End }^{-p,-q} \mathcal{V}_{\infty}, \quad p, q \geq 1, \quad \bar{G}_{p, q}=-G_{p, q}
$$

Let $\mathbf{d}=\partial^{\prime}+\partial^{\prime \prime}$ be the decomposition into the holomorphic and antiholomorphic components.

Let $\varphi_{p, q}^{a, b}$ be an End $^{-p,-q} \mathcal{V}$-valued $(a, b)$-form. We define its Hodge bigrading $(s, t)$ by setting $s:=a-p$ and $t:=b-q$. Denote by $\varphi_{s, t}$ the component of $\varphi$ of the Hodge bidegree $(-s,-t)$.

Twistor connections. Let us introduce a twistor plane $\mathbb{C}^{2}$ with coordinates $(z, w)$. Consider the product $X \times \mathbb{C}^{2}$, and the projection $\pi: X \times \mathbb{C}^{2} \rightarrow X$.

Given a datum $\left\{G_{p, q}, g_{0,0}\right\}$, we introduce a connection $\nabla_{\mathcal{G}}$ on $\pi^{*} \mathcal{V}_{\infty}$ :

$$
\nabla_{\mathcal{G}}:=\mathbf{d}+g_{0,0}+\sum_{s, t \geq 0} z^{s} w^{t}\left((s+t+1) G_{s+1, t+1}(z d w-w d z)+\left(w \partial^{\prime}-z \partial^{\prime \prime}\right) G_{s+1, t+1}\right) .
$$

The group $\mathbb{C}^{*} \times \mathbb{C}^{*}$ acts on the twistor plane: $(z, w) \longmapsto\left(\lambda_{1} z, \lambda_{2} w\right)$, as well as on the Dolbeaux complex of the local system End $\mathcal{V}$ on $X$, providing the Hodge bigrading: $\varphi_{s, t} \longmapsto \lambda_{1}^{-s} \lambda_{2}^{-t} \varphi_{s, t}$.

There is an antiholomorphic involution $\sigma:(z, w) \longmapsto(\bar{w}, \bar{z})$ of the twistor plane $\mathbb{C}^{2}$.

The connection $\nabla_{\mathcal{G}}$ has the following two basic properties:

- The connection $\nabla_{\mathcal{G}}$ is invariant under the action of the group $\mathbb{C}^{*} \times \mathbb{C}^{*}$.

- The connection $\nabla_{\mathcal{G}}$ is invariant under the composition of $\sigma$ with the complex conjugation $c$ if and only if $G_{p, q}$ are imaginary (37) and $g_{0,0}$ is real (36):

$$
(c \circ \sigma)^{*} \nabla_{\mathcal{G}}=\nabla_{\mathcal{G}} \text { if and only if } \bar{G}_{p, q}=-G_{p, q}, \quad \bar{g}_{0,0}=g_{0,0} .
$$

Restricting the connection $\nabla_{\mathcal{G}}$ to the twistor line $z+w=1$, parametrised by $z=1-u, w=u$, we get a connection

$$
\operatorname{Res}_{z+w=1} \nabla_{\mathcal{G}}:=\mathbf{d}+g_{0,0}+\sum_{s, t \geq 0}(1-u)^{s} u^{t}\left((s+t+1) G_{s+1, t+1} d u+\left(u \partial^{\prime}-(1-u) \partial^{\prime \prime}\right) G_{s+1, t+1}\right) .
$$

Definition 1.7. A connection $\nabla_{\mathcal{G}}$ as in (38) is called a twistor connection if its restriction to the twistor line $\operatorname{Res}_{z+w=1} \nabla_{\mathcal{G}}$ is flat.

It is easy to see that:

- The connection $\operatorname{Res}_{z+w=1} \nabla_{\mathcal{G}}$ is flat if and only if the datum $\left\{G_{p, q}, g_{0,0}\right\}$ satisfies a MaurerCartan system of differential equations - we call such a datum $\left\{G_{p, q}, g_{0,0}\right\}$ a Green datum.

The twistor connections form an abelian tensor category in an obvious way.

Theorem 1.8. The abelian tensor category of twistor connections is canonically equivalent to the category of variations of mixed $\mathbb{R}$-Hodge structures on $X$.

A twistor connection gives rise to a variation of mixed $\mathbb{R}$-Hodge structures on $X$ as follows. Restricting $\nabla_{\mathcal{G}}$ to $X \times\left\{\frac{1}{2}, \frac{1}{2}\right\}$ we get a flat connection $\nabla_{\mathcal{G}}^{\frac{1}{2}}$ on $\mathcal{V}_{\infty}$. We equip the local system 
$\left(\mathcal{V}_{\infty}, \nabla_{\mathcal{G}}^{\frac{1}{2}}\right)$ with a structure of a variation of mixed $\mathbb{R}$-Hodge structures. The weight filtration is the standard weight filtration on a bigraded object:

$$
W_{n} \mathcal{V}:=\oplus_{p, q \leq n} \mathcal{V}^{p, q} .
$$

Let $P$ be the operator of parallel transport for the connection $\nabla_{\mathcal{G}}$ along the line segment in the twistor plane from $X \times\{0,1\}$ to $X \times\left\{\frac{1}{2}, \frac{1}{2}\right\}$. Take the standard Hodge filtration on the bigraded object $F_{\mathrm{st}}^{p} \mathcal{V}_{\mathbb{C}}:=\oplus_{i \geq p} \mathcal{V}^{i, *}$ on the restriction of $p^{*} \mathcal{V}$ to $X \times\{0,1\}$. We define the Hodge filtration $F^{\bullet}$ on $\mathcal{V}_{\mathbb{C}}$ by applying the operator of parallel transport $P$ to the standard Hodge filtration on $p^{*} \mathcal{V}_{\mathbb{C}}$ at $\{0,1\}$ :

$$
F^{p} \mathcal{V}_{\mathbb{C}}:=P\left(F_{\mathrm{st}}^{p} \mathcal{V}_{\mathbb{C}}\right)
$$

We prove that $\left(\mathcal{V}_{\infty}, \nabla_{\mathcal{G}}^{\frac{1}{2}}, W_{\bullet}, F^{\bullet}\right)$ is a variation of real mixed Hodge structures on $X$, and that any variation is obtained this way.

Just recently M. Kapranov $[\mathrm{Ka}$ ] gave a neat interpretation of the category of mixed $\mathbb{R}$-Hodge

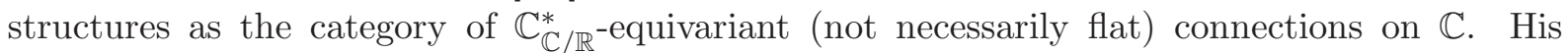
description is equivalent to the one by twistor connections when $X$ is a point. Precisely, twistor connections (38), restricted to $\mathbb{C}=\left(\mathbb{C}^{2}\right)^{\sigma}$, are natural representatives of the gauge equivalence classes of $\mathbb{C}_{\mathbb{C} / \mathbb{R}^{*}}^{*}$ equivariant connections on $\mathbb{C}$.

\subsection{The twistor transform, Hodge DG algebra, and variations of real MHS}

Theorem 1.8 describes the category of variations of mixed $\mathbb{R}$-Hodge structures via twistor connections. The next step would be to describe complexes of variations of mixed $\mathbb{R}$-Hodge structures. Precisely, we want an alternative description of the subcategory $\operatorname{Sh}_{\mathrm{Hod}}^{\mathrm{sm}}(X)$ of Saito's (derived) category $\operatorname{Sh}_{\text {Hod }}(X)$ of mixed $\mathbb{R}$-Hodge sheaves on $X$ consisting of complexes whose cohomology are smooth, i.e. are variations of mixed $\mathbb{R}$-Hodge structures. We are going to define a DG Lie coalgebra, and conjecture that the DG-category of comodules over it is a DG-enhancement of the triangulated category of smooth mixed $\mathbb{R}$-Hodge sheaves on a compact complex manifold $X$. To include the case of a smooth open complex variety $X$, one needs to impose conditions on the behavior of the forms forming the DG Lie coalgebra at infinity.

The semi-simple tensor category $\operatorname{Hod}_{X}$. Let $X$ be a regular complex projective variety. Denote by $\operatorname{Hod}_{X}$ the category of variations of real Hodge structures on $X(\mathbb{C})$. The category $\operatorname{Hod}_{X}$ is a semi-simple abelian tensor category. Denote by $w(\mathcal{L})$ the weight of a pure variation $\mathcal{L}$. The commutativity morphism is given by $\mathcal{L}_{1} \otimes \mathcal{L}_{2} \longrightarrow(-1)^{w\left(\mathcal{L}_{1}\right) w\left(\mathcal{L}_{2}\right)} \mathcal{L}_{2} \otimes \mathcal{L}_{1}$.

Hodge complexes. Given a variation $\mathcal{L}$ of real Hodge structures on $X$, we introduce a complex $\mathcal{C}_{\mathcal{H}_{\mathbb{R}}}^{\bullet}(\mathcal{L})$ and show that it calculates $\operatorname{RHom}_{\mathrm{Sh}_{\mathrm{Hod}}(X)}(\mathbb{R}(0), \mathcal{L})$ in the category of mixed $\mathbb{R}$ Hodge sheaves on $X$. We call it the Hodge complex of $\mathcal{L}$. When $\mathcal{L}=\mathbb{R}(n)$ it is the complex calculating the weight $n$ Beilinson-Deligne cohomology of $X$.

The smooth de Rham complexes of variations of real Hodge structures on $X$ can be organized into a commutative DG algebra in the category $\operatorname{Hod}_{X}$, called the de Rham DGA:

$$
\mathcal{A}_{X}:=\oplus_{\mathcal{L}} \mathcal{A}^{\bullet}(\mathcal{L}) \bigotimes \mathcal{L}^{\vee}
$$


where $\mathcal{L}$ runs through the isomorphism classes of simple objects in the category $\operatorname{Hod}_{X}$. The product is given by the wedge product of differential forms and tensor product of variations.

We define a commutative $D G$ algebra $\mathcal{D}_{X}$ in the category $\operatorname{Hod}_{X}$. As an object, it is a direct sum of complexes

$$
\mathcal{D}_{X}:=\oplus_{\mathcal{L}} \mathcal{C}_{\mathcal{H}}^{\bullet}(\mathcal{L}) \bigotimes \mathcal{L}^{\vee}
$$

where $\mathcal{L}$ runs through the isomorphism classes of simple objects in the category $\operatorname{Hod}_{X}$. To define a DGA structure on $\mathcal{D}_{X}$, we recall the projection $p: X \times \mathbb{R} \rightarrow X$, and introduce our main hero:

The twistor transform. It is a linear map

$\gamma:$ The Hodge complex of $\mathcal{V}$ on $X \longrightarrow$ The de Rham complex of $p^{*} \mathcal{V}$ on $X \times \mathbb{R}$,

Its definition is very similar to the formula (39) / (38) for the twistor connection - see Definition 4.7. The twistor transform is evidently injective, and gives rise to an injective linear map

$$
\gamma: \mathcal{D}_{X} \hookrightarrow \mathcal{A}_{X \times \mathbb{R}}
$$

Our key result in Section 4 is the following theorem.

Theorem 1.9. Let $X$ be a complex manifold. Then the image of the twistor transform (41) is closed under the differential and product, and thus is a DG subalgebra of the de Rham DGA.

Theorem 1.9 provides $\mathcal{D}_{X}$ with a structure of a commutative DGA in the category Hod . We call it the Hodge DGA of the complex variety $X$. The differential $\delta$ on $\mathcal{D}_{X}$ is obtained by conjugation by a degree-like operator $\mu$, see (106), of the natural differential on (40).

It is surprising that one can realize the Hodge DGA inside of the de Rham DGA on $X \times \mathbb{R}$.

Given a semi-simple abelian tensor category $\mathcal{P}$, denote by $D G C o m_{\mathcal{P}}$ and $D G C o L i e_{\mathcal{P}}$ the categories of DG commutative and Lie coalgebras in the category $\mathcal{P}$. Recall the two standard functors

$$
\mathcal{B}: \operatorname{DGCom}_{\mathcal{P}} \longrightarrow \text { DGCoLie } e_{\mathcal{P}}, \quad \mathcal{C}: \text { DGCoLie }_{\mathcal{P}} \longrightarrow \text { DGCom }_{\mathcal{P}} .
$$

The functor $\mathcal{B}$ is the bar construction followed by projection to the indecomposables. The functor $\mathcal{C}$ is given by the Chevalley standard complex:

$$
\left(\mathcal{G}^{\bullet}, d\right) \longmapsto \mathcal{C}(\mathcal{G}):=\left(\operatorname{Sym}^{*}\left(\mathcal{G}^{\bullet}[1]\right), \delta\right), \quad \delta:=d_{C h}+d .
$$

Applying the functor $\mathcal{B}$ to the commutative DGA $\mathcal{D}_{X}$ in the category $\operatorname{Hod}_{X}$ we get a DG Lie coalgebra $\mathcal{L}_{\mathcal{H} ; X}^{*}:=\mathcal{B}\left(\mathcal{D}_{X}\right)$ in the same category. Let $\mathcal{L}_{\mathcal{H} ; X}:=H^{0}\left(\mathcal{L}_{\mathcal{H} ; X}^{*}\right)$ be the Lie coalgebra given by its zero cohomology. We show that Theorem 1.8 is equivalent to

Theorem 1.10. The category of comodules over the Lie coalgebra $\mathcal{L}_{\mathcal{H} ; \mathcal{X}}$ in the category $\operatorname{Hod}_{X}$ is canonically equivalent to the category of variations of mixed $\mathbb{R}$-Hodge structures on $X$. The equivalence is given by the functor $\mathrm{gr}^{W}$ of the associate graded for the weight filtration.

When $X$ is a point it provides a canonical set of generators for the Lie algebra of the Hodge Galois group. The DGA $\mathcal{D}_{X}$ contains as a sub DGA the Hodge-Tate algebra of A.Levin $[\mathrm{L}$, and our description of variations of Hodge-Tate structures essentially coincides with the one in loc. cit.

Conjecture 1.11. The category $\operatorname{Sh}_{\mathrm{Hod}}^{\mathrm{sm}}(X)$ of smooth complexes of real Hodge sheaves on $X$ is equivalent to the DG-category of DG-modules over the DG Lie coalgebra $\mathcal{L}_{\mathcal{H} ; X}^{*}$. 


\subsection{Mixed $\mathbb{R}$-Hodge structure on $\pi_{1}^{\text {nil }}$ via Hodge correlators}

Recall that $\mathrm{L}_{X, S^{*}}$, see (29), is a free Lie algebra generated by $\operatorname{gr}^{W} H^{1}(X-S)$. When the data $\left(X, S, v_{0}\right)$ varies, the latter forms a variation $\mathcal{L}$ of real Hodge structures over the enhanced moduli space $\mathcal{M}_{g, n}^{\prime}, n=|S|$, (we add a tangent vector $v_{0}$ to the standard data). We are going to equip it with a Green datum, thus getting a variation mixed $\mathbb{R}$-Hodge structures.

A point $s_{0} \in X$ determines a $\delta$-current given by the evaluation of a test function at $s_{0}$. Viewed as a generalized volume form $\mu$, it provides a Green function $G(x, y)$ up to a constant. We use the tangent vector $v_{0}$ at $s_{0}$ to specify the constant. Namely, let $t$ be a local parameter at $s_{0}$ with $\left\langle d t, v_{0}\right\rangle=1$. We normalize the Green function so that $G\left(s_{0}, s\right)-\frac{1}{2 \pi i} \log |t|$ vanishes at $s=s_{0}$.

Let $\mathbf{G}_{v_{0}}$ be the corresponding Green operator (32). It is an endomorphism of the smooth bundle $\mathcal{L}_{\infty}$. Thus the construction described in Section 1.8 provides a collection of mixed $\mathbb{R}$ Hodge structures in the fibers of $\mathcal{L}$.

Furthermore, the Hodge correlators in families deliver in addition to the Green operator $\mathbf{G}_{v_{0}}$ an operator valued 1-form $g_{0,0}$. We prove in Section 7 that the pair $\mathbf{G}_{v_{0}}:=\left(\mathbf{G}_{v_{0}}, g_{0,0}\right)$ satisfies the Green data differential equations. Therefore we get a variation of mixed $\mathbb{R}$-Hodge structures.

Theorem 1.12. When the data $\left(X, S, v_{0}\right)$ varies, the variation of mixed $\mathbb{R}$-Hodge structures given by the Green datum $\mathbf{G}_{v_{0}}$ is isomorphic to the variation formed by the standard mixed $\mathbb{R}$-Hodge structures on $\pi_{1}^{\text {nil }}\left(X-S, v_{0}\right)$.

The standard MHS on $\pi_{1}^{\text {nil }}\left(X-S, v_{0}\right)$ is described by using Chen's theory of iterated integrals Ch. Our approach is different: it is given by integrals of non-holomorphic differential forms over products of copies of $X$. Theorem 1.12 implies that the two sets of periods obtained in these two descriptions coincide. For example the periods of the real MHS on $\pi_{1}^{\text {nil }}\left(\mathbb{P}^{1}-\{0,1, \infty\}, v_{0}\right)$, where $v_{0}=\partial / \partial t$ at $t=0, \mathrm{x}$ are given by the multiple $\zeta$-values [DG. So Theorem 1.12 implies that the Hodge correlators in this case are $\mathbb{Q}$-linear combinations of the multiple $\zeta$-values, which is far from being obvious from their definition.

Here is another benefit of our approach: for a modular curve $X$, it makes obvious that the Rankin-Selberg convolutions are periods of the motivic fundamental group of $X-\{$ the cusps $\}$.

\subsection{Motivic correlators on curves}

Let $X$ be a regular projective curve over a field $F, S$ a non-empty subset of $X(F)$, and $v_{0}$ a tangent vector at a point $s_{0} \in S$ defined over $F$. The motivic fundamental group of $X-S$ with the tangential base point $v_{0}$ is supposed to be a pro-unipotent Lie algebra $\mathrm{L}\left(X-S, v_{0}\right)$ in the hypothetical abelian category of mixed motives over $F$.

At the moment we have it either in realizations, $l$-adic or Hodge [D1], or when $X=\mathbb{P}^{1}$ and $F$ is a number field [DG]. We work in one of these settings, or assume the existence of the abelian category of mixed motives. A precise description of possible settings see in Section 10.2.

Since $\mathrm{L}\left(X-S, v_{0}\right)$ is a pro-object in the category of mixed motives, the Lie algebra $\mathrm{L}_{\mathrm{Mot} / \mathrm{F}}$, see Section 1.4, acts by special derivations on its associate graded for the weight filtration:

$$
\mathrm{L}_{\mathrm{Mot} / \mathrm{F}} \longrightarrow \operatorname{Der}^{S}\left(\mathrm{gr}^{W} \mathrm{~L}\left(X-S, v_{0}\right)\right) .
$$


Starting from the pure motive $\mathrm{gr}^{W} H_{1}(X-S)$ rather then from its Betti realization, and following (25), we define the motivic version $\mathcal{C} \mathcal{L} i e_{\mathcal{M} ; X, S^{*}}$ of $\mathcal{C} \mathcal{L} i e_{X, S^{*}}$. It is a Lie algebra in the semisimple abelian category of pure motives. Let $\mathcal{C} \mathcal{L} i e_{\mathcal{M} ; X, S^{*}}^{\vee}$ be the dual Lie coalgebra. Thanks to (31), there is a Lie algebra isomorphism (which gets a Tate twist in the motivic setting)

$$
\operatorname{Der}^{S}\left(\operatorname{gr}^{W} \mathrm{~L}\left(X-S, v_{0}\right)\right)=\mathcal{C} \mathcal{L} i e_{\mathcal{M} ; X, S^{*}}(-1)
$$

So dualising (42), we arrive at a map of Lie coalgebras, called the motivic correlator map:

$$
\operatorname{Cor}_{\text {Mot }}: \mathcal{C} \mathcal{L} i e_{\mathcal{M} ; X, S^{*}}^{\vee}(1) \longrightarrow \mathcal{L}_{\mathrm{Mot} / \mathrm{F}}
$$

The left hand side is decomposed into a direct sum of cyclic tensor products of simple pure motives. Their images under the motivic correlator map are called motivic correlators.

Our next goal is to relate the motivic and Hodge correlators.

\section{The canonical period map.}

Lemma 1.13. A choice of generators of the Lie algebra $\mathrm{L}_{\mathrm{Hod}}$ provides a period map

$$
p: \mathcal{L} i e_{\mathrm{Hod}} \longrightarrow i \mathbb{R} \text {. }
$$

Proof. A choice of generators of the Lie algebra $\mathrm{L}_{\mathrm{Hod}}$ is just the same thing as a projection

$$
\mathcal{L}_{i e_{\mathrm{Hod}}} \longrightarrow \oplus_{[H]} \operatorname{Ext}_{\mathbb{R}-\mathrm{MHS}}^{1}(\mathbb{R}(0), H) \otimes H^{\vee},
$$

where the sum is over the set of isomorphism classes of simple objects in the category of $\mathbb{R}$-Hodge structures. Next, for any simple object $H$ there is a canonical map

$$
p_{[H]}: \operatorname{Ext}_{\mathbb{R}-\mathrm{MHS}}^{1}(\mathbb{R}(0), H) \otimes H^{\vee} \longrightarrow i \mathbb{R} .
$$

Recall that given an $\mathbb{R}$-Hodge structure $H$, we have the formula

$$
\operatorname{Ext}_{\mathbb{R}-\mathrm{MHS}}^{1}(\mathbb{R}(0), H)=\operatorname{CoKer}\left(W_{0} H \oplus F^{0}\left(W_{0} H\right)_{\mathbb{C}} \longrightarrow\left(W_{0} H\right)_{\mathbb{C}}\right) .
$$

Thus for a pure $\mathbb{R}$-Hodge structure $H$ of Hodge degrees $(-p,-q)+(-q,-p)$, where $p, q \geq 1$, we have $\operatorname{Ext}_{\mathbb{R}-\mathrm{MHS}}^{1}(\mathbb{R}(0), H)=(H \otimes \mathbb{C}) / H=H \otimes_{\mathbb{R}} i \mathbb{R}$. So we arrive to a canonical pairing (46).

We define a period map (44) as the composition of the map (45) with the sum of the maps $p_{[H]}$. The Lemma is proved.

Therefore the canonical generators of the Lie algebra $\mathrm{L}_{\mathrm{Hod}}$, discussed in Section 1.7, give rise to a canonical period map.

Relating motivic and Hodge correlators. Let $X$ be a smooth complex curve. Just like in the case of the motivic correlators, the mixed $\mathbb{R}$-Hodge structure on $\mathrm{L}\left(X-S, v_{0}\right)$ plus the isomorphism (31) lead to the Hodge version of the motivic correlator map (43):

$$
\operatorname{Cor}_{\mathrm{Hod}}: \mathcal{C} \mathcal{L} i e_{\mathcal{H} ; X, S^{*}}^{\vee}(1) \longrightarrow \mathcal{L} i e_{\mathrm{Hod}}
$$


Here $\mathcal{C} \mathcal{L} i e_{\mathcal{H} ; X, S^{*}}^{\vee}$ is the cyclic Lie coalgebra assigned to the $\mathbb{R}$-Hodge structure $\operatorname{gr}^{W} H^{1}(X-S ; \mathbb{R})$. Forgetting the Hodge bigrading, there is an isomorphism of Lie algebras over $\mathbb{C}$

$$
\mathcal{C} \mathcal{L} i e_{\mathcal{H} ; X, S^{*}}^{\vee} \otimes \mathbb{C} \longrightarrow \mathcal{C} \mathcal{L} i e_{X, S^{*}}^{\vee}
$$

Using this, and combining (48) with the canonical period map (44) we arrive at a map

$$
\mathcal{C} \mathcal{L} i e_{X, S^{*}}^{\vee}(1) \stackrel{\text { Cor }_{\mathrm{Hod}}}{\longrightarrow} \mathcal{L} i e_{\mathrm{Hod}} \otimes \mathbb{C} \stackrel{p}{\longrightarrow} \mathbb{C} .
$$

The following result is an immediate corollary of Theorem 1.12 and the definitions.

Theorem 1.14. The composition (49) coincides with the Hodge correlator map.

Let us assume now the motivic formalism. Then the $\mathbb{R}$-Hodge realization functor provides a homomorphism of Lie coalgebras

$$
r_{\mathrm{Hod}}: \mathcal{L}_{\mathrm{Mot} / \mathbb{C}} \longrightarrow \mathcal{L}_{\mathrm{Hod}}
$$

Corollary 1.15. Assuming the motivic formalism, the complexification of the composition

$$
\mathcal{C} \mathcal{L} i e_{\mathcal{M} ; X, S^{*}}^{\vee}(1) \stackrel{\text { Cor }_{\mathrm{Mot}}}{\longrightarrow} \mathcal{L}_{\mathrm{Mot} / \mathbb{C}} \stackrel{r_{\mathrm{Hod}}}{\longrightarrow} \mathcal{L} i e_{\mathrm{Hod}} \stackrel{p}{\longrightarrow} i \mathbb{R}
$$

coincides with the Hodge correlator map. In other words, we get a commutative diagram

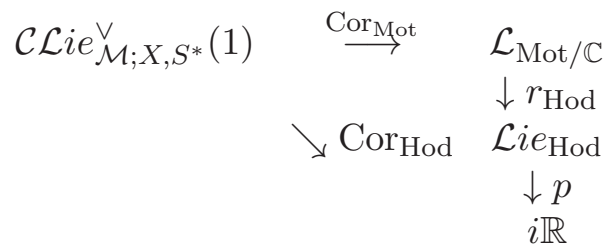

Conclusion. The source of the motivic correlator map (43) is an explicitly defined Lie coalgebra. Therefore motivic correlators come together with an explicit formula for their coproduct. The real period of the motivic correlator is the Hodge correlator. This, togerther with basic isomorphism (17), is all we need for the arithmetic analysis of the Hodge correlators.

Example: Rankin-Selberg integrals and Beilinson's elements as correlators. Here is how the story discussed in Section 1.2 fits in the correlator framework. Recall that $M$ is a modular curve, $\bar{M}$ its compactification, $a, b$ degree zero cuspidal divisors, and $g_{a}, g_{b}$ are invertible functions on $M$ whose divisors are integral multiples of $a$ and $b$. The Green function $G(a, t)$ of the divisor $a$ is an integral multiple of $\log \left|g_{a}(t)\right|^{2}$, and similarly for $G(b, t)$. Therefore integral (3) equals, up to a rational multiple, to the Hodge correlator integral provided by the cyclic word $\mathcal{C}\left(\left\{a_{0}\right\} \otimes\left\{a_{1}\right\} \otimes f(z) d z\right)$, see Fig 2 ;

$$
\operatorname{Cor}_{\mathcal{H}}\left(\left(\left\{a_{0}\right\} \otimes\left\{a_{1}\right\} \otimes f(z) d z\right)\right)=\int_{M(\mathbb{C})} G\left(a_{0}, t\right) d^{\mathbb{C}} G\left(a_{1}, t\right) \wedge f(z) d z .
$$

The latter coincides with a Rankin-Selberg integral. 
Let $M_{f}$ be the dual to the pure weight two motive corresponding to the Hecke eigenform $f(z)$. It is a direct summand of the motive $H_{1}(\bar{M})$. The motivic correlator is an element

$$
\operatorname{Cor}_{\text {Mot }}\left(\left(\left\{a_{0}\right\} \otimes\left\{a_{1}\right\} \otimes M_{f}^{\vee}\right)(1)\right) \in \mathcal{L}_{\text {Mot }} .
$$

It lies in the $M_{f}(1)^{\vee}$-isotipical component of $\mathcal{L}_{\text {Mot }}$. Since $a_{i}$ are torsion classes in the Jacobian of $\bar{M}$, we prove (Lemma 12.10) that (51) is in the kernel of the coproduct $\delta$. So, combining with the isomorphism (17), we get an element

$$
\operatorname{Cor}_{\mathrm{Mot}}\left(\left(\left\{a_{0}\right\} \otimes\left\{a_{1}\right\} \otimes M_{f}^{\vee}\right)(1)\right) \in \operatorname{Ext}_{\mathrm{Mot} / \mathbb{Q}}^{1}\left(\mathbb{Q}(0), M_{f}(1)\right) \otimes M_{f}(1)^{\vee} .
$$

Using Beilinson's conjecture [B], and the conjectural motivic Leray spectral sequence, we have

$$
\operatorname{Ext}_{\operatorname{Mot} / \overline{\mathbb{Q}}}^{1}\left(\mathbb{Q}(0), H_{1}(\bar{M})(1)\right)=K_{2}(\bar{M}) \otimes \mathbb{Q} .
$$

Indeed, for any regular projective curve $X$ over a number field $F$ with the structure map $p: X \rightarrow \operatorname{Spec}(F)$, we should have using (13), (14), and (15):

$$
K_{2}(X) \otimes \mathbb{Q}=\operatorname{Ext}_{\mathrm{Mot} / \mathrm{F}}^{2}\left(\mathbb{Q}(0), p_{*} \mathbb{Q}(2)_{X}\right)=\operatorname{Ext}_{\mathrm{Mot} / \mathrm{F}}^{1}\left(\mathbb{Q}(0), H^{1}(X)(2)\right) .
$$

To get the last isomorphism notice that $\operatorname{Ext}_{\text {Mot } / \mathrm{F}}^{i}(\mathbb{Q}(0), M)=0$ for $i \neq 1$ for any pure simple motif $M$ over a number field $F$ non-isomorphic to $\mathbb{Q}$. By Poincare duality, $H^{1}(X)=H_{1}(X)(-1)$.

This implies a similar claim for the direct summands of the motif $H^{1}(\bar{M})$.

Using (52), the motivic correlator is related to Beilinson's element (10) by the formula

$$
\operatorname{Cor}_{M o t}\left(\left(\left\{a_{0}\right\} \otimes\left\{a_{1}\right\} \otimes M_{f}^{\vee}\right)(1)\right)=\left\{g_{a}, g_{b}\right\} \otimes M_{f}(1)^{\vee} \in K_{2}(\bar{M}) \otimes M_{f}(1)^{\vee} .
$$

By Corollary 1.15, the real period map on this element is given by the Rankin-Selberg integral.

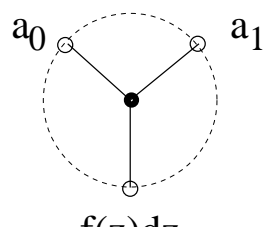

$$
f(z) d z
$$

Figure 2: The Feynman diagram for Beilinson's element.

A conjecture expressing $L\left(\mathrm{Sym}^{2} M_{f}, 3\right)$ via Hodge correlators on modular curves. Let us assume, for simplicity only, that the coefficients of the Hecke form $f(z)$ are in $\mathbb{Q}$, i.e. the motive $M_{f}$ is defined over $\mathbb{Q}$. Then Beilinson's conjecture [B] predicts that

$$
\operatorname{dim}_{\mathbb{Q}} \operatorname{Ext}_{\text {Mot } / \mathbb{Q}}^{1}\left(\mathbb{Q}(0), \operatorname{Sym}^{2} M_{f}(1)\right)=2 .
$$

Conjecture 1.16. The space $\operatorname{Ext}_{\mathrm{Mot} / \mathbb{Q}}^{1}\left(\mathbb{Q}(0), \operatorname{Sym}^{2} M_{f}(1)\right)$ is generated by the motivic correlators

$$
\operatorname{Cor}_{M o t}\left(\left(\left\{a_{1}\right\} \otimes\left\{a_{2}\right\} \otimes M_{f}^{\vee} \otimes M_{f}^{\vee}\right)(1)+\left(\left\{b_{1}\right\} \otimes M_{f}^{\vee} \otimes\left\{b_{2}\right\} \otimes M_{f}^{\vee}\right)(1)\right)
$$

for certain degree zero cuspidal divisors $a_{i}$ and $b_{i}$ on the universal modular curve, which satisfy an explicit "coproduct zero" condition. 
The two terms in (53) reflect the two different kinds of the cyclic tensor product of the following four pure motives: $\mathbb{Q}(-1), \mathbb{Q}(-1), M_{f}^{\vee}, M_{f}^{\vee}$.

Precisely, an element (53) gives rise to an element of $\operatorname{Ext}_{\mathrm{Mot} / \mathbb{Q}}^{1}\left(\mathbb{Q}(0), \operatorname{Sym}^{2} M_{f}(1)\right)$ if and only if it is killed by the coproduct in the motivic Lie coalgebra. We elaborate in Example 2 of Section 11.3 the "coproduct zero" condition for any pair of cuspidal divisors $a_{i}, b_{i}$ entering element (53). Finally, one should relate $L\left(\mathrm{Sym}^{2} M_{f}, 3\right)$ to the determinant of the $2 \times 2$ matrix, whose entries are given by the Hodge correlators of elements (53).

\subsection{Motivic multiple $L$-values}

Here are some arithmetic applications obtained by picking certain specific pairs $(X, S)$, as well as certain specific motivic correlators.

The image $\mathcal{G}\left(X-S, v_{0}\right)$ of the map (43) is called the motivic Galois Lie algebra of $X-S$. In the $l$-adic setting it is the Lie algebra of the image of the Galois group $\operatorname{Gal}\left(\bar{F} / F\left(\mu_{l^{\infty}}\right)\right)$ acting on the pro- $l$ completion $\pi_{1}^{(l)}\left(X-S, v_{0}\right)$ of the fundamental group.

Specializing to the case when $X$ is one of the following:

(i) $\mathbb{G}_{m}$, (ii) an elliptic CM curve, (iii) a Fermat curve, (iv) a modular curve, and choosing the subset $S$ appropriately, we arrive at the definition of motivic multiple $L$ values corresponding to certain algebraic Hecke characters of $\mathbb{Q}, \mathbb{Q}(\sqrt{-d}), \mathbb{Q}\left(\mu_{N}\right)$, or weight two modular forms, respectively. They span a Galois Lie coalgebra, called the multiple L-values Lie coalgebra.

In the $l$-adic setting there is one more interesting case: (v) a Drinfeld modular curve.

Given triples $\left(X^{\prime}, S^{\prime}, v_{0}^{\prime}\right)$ and $\left(X, S, v_{0}\right)$, a map $p: X^{\prime} \rightarrow X$ such that $p\left(S^{\prime}\right)=S$ and $p\left(v_{0}^{\prime}\right)=v_{0}$ induces a map of the motivic fundamental Lie algebras $p: \mathrm{L}\left(X^{\prime}-S^{\prime}, v_{0}^{\prime}\right) \rightarrow \mathrm{L}\left(X-S, v_{0}\right)$, and hence a map of the Galois Lie algebras $p: \mathcal{G}\left(X^{\prime}-S^{\prime}, v_{0}^{\prime}\right) \rightarrow \mathcal{G}\left(X-S, v_{0}\right)$, and the dual map of the Galois Lie coalgebras.

In each of the cases (i)-(v), the open curves $X-S$ form a tower. For instance in the case (iv) it is the modular tower. Therefore the corresponding Galois Lie coalgebras form an inductive system. In many cases there is a canonical "averaged tangential base point" (Section 10.4), so below we ignore the base point. There is an adelic description of the limiting Galois Lie coalgebras. It is given in terms of the corresponding base field in the first three cases, and via the automorphic representations attached to the weight two modular forms in the modular curve case. In the latter case there is a generalization dealing with modular forms of arbitrary integral weight $k \geq 2$, which we will elaborate elsewhere.

Here is a description of the pairs $(X, S)$ and the corresponding towers.

1. Let $X=\mathbb{P}^{1}$, and $S$ is an arbitrary subset of $\mathbb{P}^{1}$. This is the situation studied in [G7], G9].

a) A very interesting case is when $S=\{0\} \cup\{\infty\} \cup \mu_{N}$. It was studied in [G4], [DG].

The tower. The curves $\mathbb{G}_{m}-\mu_{N}$, parametrized by positive integers $N$, form a "tower" for the isogenies $\mathbb{G}_{m}-\mu_{M N} \rightarrow \mathbb{G}_{m}-\mu_{N}$.

2. The curve is an elliptic curve $E$, and $S$ is any subset. An interesting special case is when $S=E[N]$ is the subgroup of $N$-torsion points of $E$. The tower in this case is formed by the isogenies $E-E[M N] \rightarrow E-E[N]$. 
There are the following ramifications:

a) The universal elliptic curve $\mathcal{E}$ with the level $N$ structure over the modular curve.

s) The curve $E$ is a $C M$ curve, $\mathcal{N}$ an ideal in the endomorphism ring $\operatorname{End}(E)$, and $S$ is the subgroup $E[\mathcal{N}]$ of the $\mathcal{N}$-torsion points.

3. The curve is the Fermat curve $\mathbb{F}_{N}$, given in $\mathbb{P}^{2}$ by the equation $x^{N}+y^{N}=z^{N}$, and $S$ is the intersection of $\mathbb{F}_{N}$ with the coordinate triangle $x=0, y=0, z=0$ in $\mathbb{P}^{2}$.

The tower. It is given by the maps $\mathbb{F}_{M N} \rightarrow \mathbb{F}_{N},(x, y, z) \longmapsto\left(x^{M}, y^{M}, z^{M}\right)$.

4. The curve $X=X(N)$ is the level $N$ modular curve, $S$ is the set of the cusps. There is a tower of modular curves, and especially interesting situation appears at the limit, i.e. when $\mathcal{M}:=\lim _{\longleftarrow} X(N)$ is the universal modular curve and $S$ is the set of its cusps.

5. Let $A$ be the ring of rational functions on a regular projective curve over a finite field, which are regular at a chosen point $\infty$ of the curve. Let $I$ be an ideal in $A$. The Drinfeld modular curve is the moduli space of the rank 2 elliptic modules with the $I$-level structure. Take it as our curve $X$, and let $S$ is the set of the cusps. There is a tower of the Drinfeld modular curves. In the limit we get the universal Drinfeld modular curve.

In each of these cases we get a supply of elements of the motivic Lie coalgebras. These are

1. "Cyclic" versions of motivic multiple polylogarithms.

a) Motivic multiple $L$-values for the Dirichlet characters of $\mathbb{Q}$.

2. Motivic multiple elliptic polylogarithms, and their restrictions to the torsion points.

a) Higher (motivic) analogs of modular units.

b) Motivic multiple $L$-values for Hecke Grössencharacters of imaginary quadratic fields.

3. Motivic multiple $L$-values for the Jacobi sums Grössencharacters of $\mathbb{Q}\left(\mu_{N}\right)$.

4. Motivic multiple $L$-values for the modular forms of weight 2 .

5. Similar objects in the function field case.

We prove in Section 11 that the real periods of the motivic multiple elliptic polylogarithms are the generalized Eisenstein-Kronecker series, defined in G1] as integrals of the classical EisensteinKronecker series, and obtain similar results in the rational case.

What are the multiple $L$-values? In each of the above cases the motivic multiple $L$-values are certain natural collections of elements of the motivic Lie coalgebra, closed under the Lie cobracket. Their periods are numbers, called the multiple $L$-values.

The "classical" motivic $L$-values are the elements killed by the Lie cobracket. Moreover, they are precisely the cogenerators of the corresponding motivic Lie coalgebra, providing the name for the latter. Their periods are special values of $L$-functions.

The "classical" motivic $L$-values are motivic cohomology classes. In general the motivic multiple $L$-values are no longer motivic cohomology classes. 
Example. The first multiple $L$-values discovered were Euler's multiple $\zeta$-numbers

$$
\zeta\left(n_{1}, \ldots, n_{m}\right)=\sum_{0<k_{1}<\cdots<k_{m}} \frac{1}{k_{1}^{n_{1}} \ldots k_{m}^{n_{m}}} .
$$

The motivic $\zeta$-values are the elements $\zeta_{\mathcal{M}}(2 n-1) \in \operatorname{Ext}_{\operatorname{Mot} / \mathbb{Z}}^{1}(\mathbb{Q}(0), \mathbb{Q}(n)$. The latter Extgroup is one-dimensional, and is generated by $\zeta_{\mathcal{M}}(2 n-1)$. The motivic multiple zeta values $\zeta_{\mathcal{M}}\left(n_{1}, \ldots, n_{m}\right)$ are the elements of the motivic Lie coalgebra of the category of mixed Tate motives over $\operatorname{Spec}(\mathbb{Z})$. The conjecture that they span the latter was recently proved by Brown $\mathrm{Br}$.

Similarly, each motivic multiple $L$-values Lie coalgebra is tied up to a certain subcategory of the category of mixed motives. A very interesting question is how far it is from the motivic Lie coalgebra which governs that mixed category. See [G4 for the case 1a) and G10] for the case $2 \mathrm{~b}$ ), where the gap in each case was related to the geometry of certain modular varieties.

In all above cases the motivic cohomology group responsible for the $L$-values were onedimensional.

Question. Can one define multiple $L$-values in a more general situation?

\subsection{Coda: Feynman integrals and motivic correlators}

We suggested in Section 8 of [G7] the following picture relating Feynman integrals and mixed motives. If correlators of a Feynman integral are periods, they should be upgraded to motivic correlators, which lie in the motivic Lie coalgebra, and whose periods are the Feynman correlators. The obtained motivic correlators should be closed under the coproduct in the motivic Lie coalgebra - otherwise the original set of Feynman correlators was not complete. Finally, the coproduct of the motivic correlators should be calculated as follows. There should be a combinatorially defined Lie coalgebra - the renormalization Lie coalgebra - and the motivic correlators should provide a homomorphism

$$
\text { the renormalization Lie coalgebra } \longrightarrow \text { the motivic Lie coalgebra. }
$$

This paper gives an example of realization of this program. Indeed, the Hodge correlators are the tree level correlators of the Feynman integral from Section 1.7. We upgrade them to motivic correlators. They span a Lie coalgebra, which is nothing else but the Galois Lie coalgebra $\mathcal{G}\left(X-S, v_{0}\right)$. The Lie coalgebra $\mathcal{C} \mathcal{L} i e_{X, S^{*}}^{\vee}$ plays the role of the renormalization Lie coalgebra: There is a canonical Lie coalgebra homomorphism $\mathcal{C} \mathcal{L} i e_{X, S^{*}}^{\vee} \rightarrow \mathcal{G}\left(X-S, v_{0}\right)$ describing the coproduct of motivic correlators, which we think of as the homomorphism (54)).

Problem. Develop a similar picture for Feynman integrals whose correlators are periods.

\subsection{The structure of the paper}

The paper is build from four parts. Their architecture is described as follows.

1. In Section 2 we introduce a key tool to define Hodge correlators: a polydifferential operator $\omega_{m}$. Its properties become evident in its twistor version, $\widehat{\omega}_{m}$. 
Using the operator $\omega_{m}$, and the sum of the plane decorated trees construction, we define in Section 3 Hodge correlators on punctured curves.

We show in Section 13 that the Hodge correlators on punctured curves indeed are correlators of a Feynman integral, i.e. can be defined via a Feynman integral procedure. However we do not use this anywhere else in the paper.

2. In Section 4 we define a Hodge complex of a variation of Hodge structures. We introduce a commutative DGA structure on the sum of Hodge complexes over isomorphism classes of simple variations of Hodge structures on a complex manifold $X$. We call it the Hodge DGA of $X$. We define a twistor transform and show that it embeds the Hodge DGA of $X$ into the De Rham DGA of $X \times \mathbb{R}$. This elucidates the definition of the Hodge DGA.

In Section 5 we show that variations of mixed Hodge structures are described by twistor connections. The flatness of the twistor connection encodes the Griffith transversality. It amounts to Maurer-Cartan type differential equations on the Green data describing a twistor connection.

3. In Section 6, given a symplectic vector space $H$ and a set $S$, we define a Lie algebra $\mathcal{C}_{H, S}$. It generalizes the Lie algebras defined in [G4, [G9] in the case $H=0$. We introduce an $L_{\infty}$-algebra of plane trees decorated by $H \oplus \mathbb{Q}[S]$. It is a resolution of the Lie algebra $\mathcal{C}_{H, S}$.

The Lie algebra $\mathcal{C}_{H, S}$ has a Lie subalgebra $\mathcal{C} \mathcal{L} i e_{H, S}$.

Our basic example is assigned to a smooth family of compact curves $p: X \rightarrow B$ with a smooth divisor $S \rightarrow B$, and a base point $s_{0} \in S$. Let $S^{*}:=S-s_{0}$. The Lie algebra $\mathcal{C} \mathcal{L} i e_{H, S^{*}}$ assigned to $H=R_{1} p_{*}(X, \mathbb{R})$ is denoted by $\mathcal{C} \mathcal{L} i e_{X, S^{*}}$.

In Section 7, given such a family $p: X \rightarrow B$, we define the Hodge correlator twistor connection $\nabla_{\mathbf{G}}$ on $B \times \mathbb{C}^{2}$. It takes values in a variation of Lie algebras $\mathcal{C} \mathcal{L} i e_{X, S^{*}}$. The Hodge correlators defined in Section 3 are just the coefficients of the twistor connection $\nabla_{\mathbf{G}}$. The differential equations for the Hodge correlators express the fact that the restriction of the twistor connection to $B \times \mathbb{C}$, where $\mathbb{C} \subset \mathbb{C}^{2}$ is the twistor line $z+w=1$, is flat.

We stress that our construction in Section 7 delivers the Hodge correlator twistor connection $\nabla_{\mathbf{G}}$ directly, using the twistor operator $\widehat{\omega}_{m}$ and the sum over all plane trivalent trees construction. So the very notion of twistor connection is naturally build in the definition of the Hodge correlators.

4. In Section 8 we identify the Lie algebra $\mathcal{C} \mathcal{L} i e_{X, S^{*}}$ with the Lie algebra of special derivations of $\mathrm{gr}^{W} \pi_{1}^{\text {nil }}\left(X-S, v_{0}\right)$, where $v_{0}$ is a tangent vector at $s_{0}$. Since the Hodge correlator connection $\nabla_{\mathbf{G}}$ lies in $\mathcal{C} \mathcal{L} i e_{X, S^{*}}$, it gives rise to a special derivation $\mathbf{G}$ of the Lie algebra $\mathrm{gr}^{W} \pi_{1}^{\text {nil }}\left(X-S, v_{0}\right)$. By the Section 5 construction, it provides a real mixed Hodge structure on the variation $\pi_{1}^{\text {nil }}\left(X-S, v_{0}\right)$ over $B$. In Section 9 we prove that it is the standard one.

In Section 10 we translate results of Section 8 into the motivic framework. Using this we introduce motivic correlators. They are canonical elements in the motivic Lie coalgebra. The Hodge correlators are the canonical real periods of the motivic correlators.

In Section 11 we consider examples. We show how the classical and elliptic polylogarithms appear as Hodge correlators for simple Feynman diagrams. We prove that for an elliptic curve the Hodge correlators can be expressed by the multiple Eisenstein-Kronecker series. 
In Section 12 we show that Hodge / motivic correlators on modular curves generalize the Rankin-Selberg integrals / Beilinson's elements in $K_{2}$ of modular curves. Going to the limit in the tower of modular curves we get an automorphic adelic description of the correlators on modular curves.

Acknowledgments. I am very grateful to A.A. Beilinson for many fruitful discussions, and especially for encouragement over the years to work on Hodge correlators.

Many ideas of this work were worked out, and several Sections written, during my stays at the MPI(Bonn) and IHES. A part of this work was written during my stay at the Okayama University (Japan) in May 2005. I am indebted to Hiraoki Nakamura for the hospitality there. I am grateful to these institutions for the hospitality and support. I was supported by the NSF grants DMS-0400449, DMS-0653721, DMS-1059129 and DMS-1301776. I am very grateful to referees for the extraordinary job. I am grateful to C. Hertling for pointing out some errors, and sending me notes of his seminar talks in 2008-2009 on the paper.

\section{Polydifferential operator $\omega_{m}$}

In Section 2 we introduce a polydifferential operator $\omega_{m}\left(\varphi_{1}, \ldots, \varphi_{m}\right)$.

In Section 2.1 we present a down to earth definition of the polydifferential operator $\omega_{m+1}$ on the Dolbeaut complex of a complex manifold. The definition extends naturally to a cohomological Dolbeaut bicomplex, axiomatizing the Dolbeaut bicomplex of a variation of Hodge structures.

A much better definition of a twistor cousin $\widehat{\omega}_{m}$ of the operator $\omega_{m}$ is given in Section 2.2 . The twistor approach is more conceptual, and allows to give transparent proofs of the main properties of the operator $\omega_{m}$. However since it looks a bit out of the bleu we include both.

The twistor approach makes evident the two main features of the operator $\omega_{m}$ :

- The differential equation the operator $\omega_{m}$ satisfies.

- The multiplicativity property of the operator $\omega_{m}$.

Namely, let $\mathcal{A}^{*, *}(M)$ be the Dolbeaut bicomplex of a complex manifold $M$. Then the two operators fit into the following commutative diagram, where $\int$ is the integration over the line segment connecting the points $(1,0)$ and $(0,1)$ in $\mathbb{C}^{2}$ :

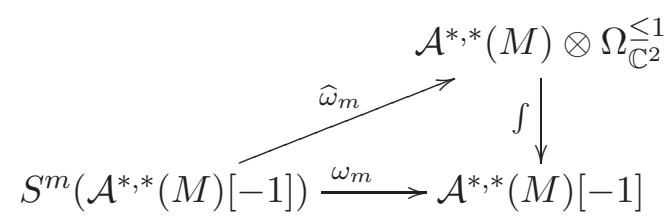

The map $\widehat{\omega}_{m}$ is a graded algebra map. It is easy to prove a differential equation it satisfies.

The integration over a segment almost commutes with the the differential: there are additional boundary terms. This implies the differential equation for the operator $\omega_{m}$. 


\subsection{The form $\omega_{m}\left(\varphi_{1}, \ldots, \varphi_{m}\right)$ and its properties}

Given a function $F\left(f_{1}, \ldots, f_{m}\right)$ of certain variables $f_{i}$ of degrees $\left|f_{i}\right|$, set

$$
\operatorname{Sym}_{m} F\left(f_{1}, \ldots, f_{m}\right):=\sum_{\sigma \in \Sigma_{m}} \operatorname{sgn}_{\sigma ; f_{1}, \ldots, f_{m}} F\left(f_{\sigma(1)}, \ldots, f_{\sigma(m)}\right)
$$

where the sign of the transposition of $f_{1}, f_{2}$ is $(-1)^{\left(\left|f_{1}\right|+1\right)\left(\left|f_{2}\right|+1\right)}$, and the sign of a permutation written as a product of transpositions is the product of the signs of the transpositions. It is the symmetrisation of elements $\bar{f}_{i}$ obtained by shifting the degree of $f_{i}$ by one: $\operatorname{deg}\left(\bar{f}_{i}\right)=\operatorname{deg}\left(f_{i}\right)+1$.

Definition 2.1. Let $\varphi_{0}, \ldots, \varphi_{m}$ be any $m+1$ smooth forms on a complex manifold $M$. Set

$$
\omega_{m+1}\left(\varphi_{0}, \ldots, \varphi_{m}\right):=\frac{1}{(m+1) !} \operatorname{Sym}_{m+1}\left(\sum_{k=0}^{m}(-1)^{k} \varphi_{0} \wedge \partial^{\prime} \varphi_{1} \wedge \ldots \wedge \partial^{\prime} \varphi_{k} \wedge \partial^{\prime \prime} \varphi_{k+1} \wedge \ldots \wedge \partial^{\prime \prime} \varphi_{m}\right) .
$$

Here we write the deRham differential $d$ on $M$ as a sum of its holomorphic and antiholomorphic components $d=\partial^{\prime}+\partial^{\prime \prime}$. We apply the symmetrisation (55) to a function of $\varphi_{0}, \ldots, \varphi_{m}$.

Let $\mathcal{A}_{M}^{k}$ be the space of smooth $k$-forms on $M$. We use the notation $\circ$ for the symmetric product. The map (56) gives rise to a degree zero linear map

$$
\omega_{m+1}: S^{m+1}\left(\mathcal{A}_{M}^{\bullet}[-1]\right) \longrightarrow \mathcal{A}_{M}^{\bullet}[-1], \quad \omega_{m+1}: \varphi_{0} \circ \ldots \circ \varphi_{m} \longmapsto \omega_{m+1}\left(\varphi_{0}, \ldots, \varphi_{m}\right) .
$$

Example 2.2. One has $\omega_{1}\left(\varphi_{0}\right)=\varphi_{0}$,

$$
\omega_{2}\left(\varphi_{0}, \varphi_{1}\right)=\frac{1}{2}\left(\varphi_{0} \wedge \partial^{\prime \prime} \varphi_{1}-\varphi_{0} \wedge \partial^{\prime} \varphi_{1}+(-1)^{\left(\left|\varphi_{0}\right|+1\right)\left(\left|\varphi_{1}\right|+1\right)}\left(\varphi_{1} \wedge \partial^{\prime \prime} \varphi_{0}-\varphi_{1} \wedge \partial^{\prime} \varphi_{0}\right)\right) .
$$

The first key property of this map is the differential equation it satisfies:

\section{Lemma 2.3.}

$$
\begin{aligned}
& d \omega_{m+1}\left(\varphi_{0}, \ldots, \varphi_{m}\right)=\frac{1}{m !} \operatorname{Sym}_{m+1}\left((-1)^{\left|\varphi_{0}\right|} \partial^{\prime \prime} \partial^{\prime} \varphi_{0} \wedge \omega_{m}\left(\varphi_{1}, \ldots, \varphi_{m}\right)\right)+ \\
& (-1)^{m} \partial^{\prime} \varphi_{0} \wedge \ldots \wedge \partial^{\prime} \varphi_{m}+\partial^{\prime \prime} \varphi_{0} \wedge \ldots \wedge \partial^{\prime \prime} \varphi_{m} .
\end{aligned}
$$

(The first term can be written as $\sum_{j=0}^{m}(-1)^{j} \pm \partial^{\prime \prime} \partial^{\prime} \varphi_{j} \wedge \omega_{m}\left(\varphi_{0}, \ldots, \widehat{\varphi}_{j}, \ldots, \varphi_{m}\right)$ ).

Below we give a direct proof of Lemma 2.3. A conceptual proof, which requires almost no calculations, is presented in Section 2.2. The reader can skip the direct proof.

Proof. There are the following three identities, which allow to move differential operators under the alternation sign, without moving the forms $\varphi_{i}$ :

$$
\begin{gathered}
\operatorname{Sym}_{2}\left(\partial^{\prime \prime} \varphi_{1} \wedge \partial^{\prime} \varphi_{2}\right)=\operatorname{Sym}_{2}\left(\partial^{\prime} \varphi_{1} \wedge \partial^{\prime \prime} \varphi_{2}\right) . \\
\operatorname{Sym}_{2}\left((-1)^{\left|\varphi_{1}\right|} \varphi_{1} \wedge \partial^{\prime} \partial^{\prime \prime} \varphi_{2}\right)=-\operatorname{Sym}_{2}\left((-1)^{\left|\varphi_{1}\right|} \partial^{\prime} \partial^{\prime \prime} \varphi_{1} \wedge \varphi_{2}\right) . \\
\operatorname{Sym}_{2}\left(\partial^{\prime} \varphi_{1} \wedge \partial^{\prime \prime} \partial^{\prime} \varphi_{2}\right)=\operatorname{Sym}_{2}\left((-1)^{\left|\varphi_{1}\right|+1} \partial^{\prime \prime} \partial^{\prime} \varphi_{1} \wedge \partial^{\prime} \varphi_{2}\right) .
\end{gathered}
$$

Indeed, identity (58) is proved as follows:

$$
\operatorname{Sym}_{2}\left(\partial^{\prime \prime} \varphi_{1} \wedge \partial^{\prime} \varphi_{2}\right)=(-1)^{\left(\left|\varphi_{1}\right|+1\right)\left(\left|\varphi_{2}\right|+1\right)} \operatorname{Sym}_{2}\left(\partial^{\prime \prime} \varphi_{2} \wedge \partial^{\prime} \varphi_{1}\right)=\operatorname{Sym}_{2}\left(\partial^{\prime} \varphi_{1} \wedge \partial^{\prime \prime} \varphi_{2}\right) .
$$


Identity (59) is proved by

$$
\begin{gathered}
\operatorname{Sym}_{2}\left((-1)^{\left|\varphi_{1}\right|} \varphi_{1} \wedge \partial^{\prime} \partial^{\prime \prime} \varphi_{2}\right)=\operatorname{Sym}_{2}\left((-1)^{\left|\varphi_{1}\right|+\left|\varphi_{1}\right|\left|\varphi_{2}\right|} \partial^{\prime} \partial^{\prime \prime} \varphi_{2} \wedge \varphi_{1}\right) \\
=\operatorname{Sym}_{2}\left((-1)^{\left|\varphi_{2}\right|+\left|\varphi_{2}\right|\left|\varphi_{1}\right|+\left(\left|\varphi_{1}\right|+1\right)\left(\left|\varphi_{2}\right|+1\right)} \partial^{\prime} \partial^{\prime \prime} \varphi_{1} \wedge \varphi_{2}\right)=-\operatorname{Sym}_{2}\left((-1)^{\left|\varphi_{1}\right|} \partial^{\prime} \partial^{\prime \prime} \varphi_{1} \wedge \varphi_{2}\right) .
\end{gathered}
$$

The third identity is checked in a similar way. These formulas are easily generalised to $\mathrm{Sym}_{m}$.

We begin by computing $d \omega_{m+1}\left(\varphi_{0}, \ldots, \varphi_{m}\right)$ as

$$
\begin{aligned}
& d \omega_{m+1}\left(\varphi_{0}, \ldots, \varphi_{m}\right)= \\
& \frac{1}{(m+1) !} \operatorname{Sym}_{m+1}\left(\sum_{k=0}^{m}(-1)^{k} \cdot\left(\partial^{\prime} \varphi_{0}+\partial^{\prime \prime} \varphi_{0}\right) \wedge \partial^{\prime} \varphi_{1} \wedge \ldots \wedge \partial^{\prime} \varphi_{k} \wedge \partial^{\prime \prime} \varphi_{k+1} \wedge \ldots \wedge \partial^{\prime \prime} \varphi_{m}+\right. \\
& \sum_{k=1}^{m}(-1)^{k+\left|\varphi_{0}\right|} k \cdot \varphi_{0} \wedge \partial^{\prime \prime} \partial^{\prime} \varphi_{1} \wedge \partial^{\prime} \varphi_{2} \wedge \ldots \wedge \partial^{\prime} \varphi_{k} \wedge \partial^{\prime \prime} \varphi_{k+1} \wedge \ldots \wedge \partial^{\prime \prime} \varphi_{m}+ \\
& \left.\sum_{k=0}^{m-1}(-1)^{\left|\varphi_{0}\right|+\ldots+\left|\varphi_{k}\right|}(m-k) \cdot \varphi_{0} \wedge \partial^{\prime} \varphi_{1} \wedge \ldots \wedge \partial^{\prime} \varphi_{k} \wedge \partial^{\prime} \partial^{\prime \prime} \varphi_{k+1} \wedge \partial^{\prime \prime} \varphi_{k+2} \wedge \ldots \wedge \partial^{\prime \prime} \varphi_{m}\right) .
\end{aligned}
$$

Using (58), the first term contributes to the first term in the right hand side of (57).

Using (60), the third term is written as

$\frac{1}{(m+1) !} \operatorname{Sym}_{m+1}\left(\sum_{k=0}^{m-1}(m-k)(-1)^{k+\left|\varphi_{0}\right|} \cdot \varphi_{0} \wedge \partial^{\prime} \partial^{\prime \prime} \varphi_{1} \wedge \partial^{\prime} \varphi_{2} \wedge \ldots \wedge \partial^{\prime} \varphi_{k+1} \wedge \partial^{\prime \prime} \varphi_{k+2} \wedge \ldots \wedge \partial^{\prime \prime} \varphi_{m}\right)$.

Using (59) and $\partial^{\prime} \partial^{\prime \prime}=-\partial^{\prime \prime} \partial^{\prime}$, the last two terms in the expression for $d \omega_{m+1}\left(\varphi_{0}, \ldots, \varphi_{m}\right)$ give

$$
\begin{aligned}
& \frac{1}{(m+1) !} \operatorname{Sym}_{m+1}\left(\sum_{k=1}^{m}(-1)^{k-1+\left|\varphi_{0}\right|} k \cdot \partial^{\prime \prime} \partial^{\prime} \varphi_{0} \wedge \varphi_{1} \wedge \partial^{\prime} \varphi_{2} \wedge \ldots \wedge \partial^{\prime} \varphi_{k} \wedge \partial^{\prime \prime} \varphi_{k+1} \wedge \ldots \wedge \partial^{\prime \prime} \varphi_{m}\right. \\
& \left.\quad+\sum_{k=0}^{m-1}(-1)^{k+\left|\varphi_{0}\right|}(m-k) \cdot \partial^{\prime \prime} \partial^{\prime} \varphi_{0} \wedge \varphi_{1} \wedge \partial^{\prime} \varphi_{2} \wedge \ldots \wedge \partial^{\prime} \varphi_{k+1} \wedge \partial^{\prime \prime} \varphi_{k+2} \wedge \ldots \wedge \partial^{\prime \prime} \varphi_{m}\right) .
\end{aligned}
$$

Changing the summation in the second term from $0 \leq k \leq m-1$ to $1 \leq k \leq m$, and using $k+(m+1-k)=m+1$, we see that it coincides with the term containing Laplacians in (57).

Remark. Let $f_{i}$ be rational functions on a complex algebraic variety. Let $\varphi_{i}:=\log \left|f_{i}\right|$. Then the form (56) is a part of a cocycle representing the product in real Deligne cohomology of 1-cocycles $\left(\log \left|f_{i}\right|, d \log f_{i}\right)$ representing classes in $H_{\mathcal{D}}^{1}(X, \mathbb{R}(1))$. The form (56) in this set up was used for the definition of Chow polylogarithms and, more generally, in the construction of the canonical regulator map on motivic complexes [G2], G8].

\subsection{Twistor definition of the operator $\omega_{m}$.}

Definition 2.4. A cohomological Dolbeaut complex $\left(\mathcal{A}^{*, *}, \partial^{\prime}, \partial^{\prime \prime}\right)$ is given by the following data:

- $A$ vector space $\mathcal{A}^{*, *}$ with a Hodge bigrading. 
- A cohomological grading and a differential $\mathbf{d}$ of cohomological degree 1 , written as

$$
\mathbf{d}=\partial^{\prime}+\partial^{\prime \prime}
$$

where $\partial^{\prime}$ and $\partial^{\prime \prime}$ have the cohomological degree 1 , and the Hodge bidegrees $(1,0)$ and $(0,1)$.

- $\left(\mathcal{A}^{*, *}, \partial^{\prime}, \partial^{\prime \prime}\right)$ satisfies the $\partial^{\prime} \partial^{\prime \prime}$-lemma.

Decomposing $\mathbf{d}^{2}=0$ into the components of Hodge bidegrees $(2,0),(1,1)$ and $(0,2)$ we get

$$
\partial^{\prime 2}=\partial^{\prime \prime 2}=\partial^{\prime} \partial^{\prime \prime}+\partial^{\prime \prime} \partial^{\prime}=0 .
$$

We set

$$
\mathbf{d}^{\mathbb{C}}:=\partial^{\prime}-\partial^{\prime \prime}
$$

An example of a cohomological Dolbeaut bicomplex is given by the Dolbeaut bicomplex of a complex manifold. A more general example is given by the Dolbeaut bicomplex of a variation of Hodge structures, discussed in Section 4 .

Although the $\partial^{\prime} \partial^{\prime \prime}$-lemma is essential, we do not use it in Section 2.

The twistor polydifferential operator $\widehat{\omega}_{m}$. Let $\mathbb{C}^{2}$ be the twistor plane with canonical coordinates $(z, w)$. The group $\mathbb{C}^{*} \times \mathbb{C}^{*}$ acts on the twistor plane and on the bicomplex $\mathcal{A}^{*, *}$ by

$$
(\lambda, \mu):(z, w) \longmapsto(\lambda z, \mu w), \quad(\lambda, \mu): f_{s, t} \in \mathcal{A}^{s, t} \longmapsto \lambda^{s} \mu^{t} f_{s, t} .
$$

So the Hodge bidegree can be defined as the character of the action of the group $\mathbb{C}^{*} \times \mathbb{C}^{*}$.

Take the complex vector space $\left\langle\partial^{\prime}, \partial^{\prime \prime}\right\rangle_{\mathbb{C}}$ spanned by the operators $\partial^{\prime}$ and $\partial^{\prime \prime}$. Then the map

$$
\left\langle\partial^{\prime}, \partial^{\prime \prime}\right\rangle_{\mathbb{C}} \otimes \mathcal{A}^{*, *} \longrightarrow \mathcal{A}^{*, *}, \quad \partial^{\prime} \otimes f \longmapsto \partial^{\prime} f, \quad \partial^{\prime \prime} \otimes f \longmapsto \partial^{\prime \prime} f
$$

is $\mathbb{C}^{*} \times \mathbb{C}^{*}$-equivariant for the following action of the group $\mathbb{C}^{*} \times \mathbb{C}^{*}$ on the operators $\partial^{\prime}, \partial^{\prime \prime}$ :

$$
(\lambda, \mu):\left(\partial^{\prime}, \partial^{\prime \prime}\right) \longmapsto\left(\lambda \partial^{\prime}, \mu \partial^{\prime \prime}\right) .
$$

The space $\left\langle\partial^{\prime}, \partial^{\prime \prime}\right\rangle_{\mathbb{C}}$ is identified canonically, as $\mathbb{C}^{*} \times \mathbb{C}^{*}$-module, with the space of linear functions $(z, w)$ on the twistor plane, "explaning" the role of the latter.

Consider the tensor product of the complex $\mathcal{A}^{*, *}$ with the algebraic de Rham complex of $\mathbb{C}^{2}$;

$$
\mathcal{A}^{*, *} \otimes_{\mathbb{C}} \Omega_{\mathbb{C}^{2}}^{\bullet}, \mathbf{d}+\mathrm{d}_{\mathbb{C}^{2}} .
$$

Let us introduce the twistor version of the operator $\mathbf{d}^{\mathbb{C}}$ :

$$
\mathrm{D}^{\mathbb{C}}:=w \partial^{\prime}-z \partial^{\prime \prime}+(z d w-w d z) .
$$

It has cohomological degree +1 and Hodge bidegree $(1,1)$.

Denote by $\mathbb{C}(-1)[-1]$ a one dimensional vector space of Hodge bidegree $(1,1)$ and cohomological degree +1 . So $\mathcal{A}^{*, *}(-1)[-1]$ is $\mathcal{A}^{*, *}$ with each of its three degrees shifted by one.

Definition 2.5. The twistor operator $\widehat{\omega}_{m}$ is the following map:

$$
\begin{aligned}
& \widehat{\omega}_{m}: S^{m}\left(\mathcal{A}^{*, *}(-1)[-1]\right) \longrightarrow \mathcal{A}^{*, *} \otimes \Omega_{\mathbb{C}^{2}}^{\leq 1} \\
& \widehat{\omega}_{m}\left(\varphi_{1}, \ldots, \varphi_{m} ; z, w\right):=\mathrm{D}^{\mathbb{C}} \varphi_{1} \wedge \ldots \wedge \mathrm{D}^{\mathbb{C}} \varphi_{m} .
\end{aligned}
$$


Lemma 2.6. The twistor operator $\widehat{\omega}_{m}$ in (64) preserves each of the three degrees.

It is a graded algebra homomorphism:

$$
\widehat{\omega}_{m+n}\left(\varphi_{1}, \ldots, \varphi_{m+n} ; z, w\right)=\widehat{\omega}_{m}\left(\varphi_{1}, \ldots, \varphi_{m} ; z, w\right) \wedge \widehat{\omega}_{n}\left(\varphi_{m+1}, \ldots, \varphi_{m+n} ; z, w\right) .
$$

Proof. Since the $\mathrm{D}^{\mathbb{C}}$ has cohomological degree +1 and Hodge bidegree $(1,1)$, the operator $\widehat{\omega}_{m}$ preserves each of the three degrees. The second claim is clear.

The next question is how the differential acts on the map $\widehat{\omega}_{m}$. The answer is most naturally given by using the restriction to the twistor line.

Restriction to the twistor line and the differential. Let us consider the twistor line:

$$
\mathbb{C}:=\left\{z, w \in \mathbb{C}^{2} \mid z+w=1\right\} .
$$

It is parametrised by

$$
z=1-u, \quad w=u \text {. }
$$

We denote by $\left(\Omega_{\mathbb{C}}^{\bullet}, \mathrm{d}_{u}\right)$ the algebraic de Rham complex of the twistor line. Observe that

$$
\operatorname{Res}_{z+w=1}(z d w-w d z)=d u .
$$

Denote by $\widehat{\omega}_{m}\left(\varphi_{1}, \ldots, \varphi_{m} ; u\right)$ the restriction of $\widehat{\omega}_{m}\left(\varphi_{1}, \ldots, \varphi_{m} ; z, w\right)$ to the twistor line. Let $\mathbf{d}+\mathrm{d}_{u}$ be the total differential on $\mathcal{A}^{*, *} \otimes \Omega_{\mathbb{C}}^{\bullet}$.

Lemma 2.7. One has

$$
\begin{aligned}
& \left(\mathbf{d}+\mathrm{d}_{u}\right) \widehat{\omega}_{m}\left(\varphi_{1}, \ldots, \varphi_{m} ; u\right)=\partial^{\prime \prime} \partial^{\prime} \varphi_{1} \wedge \mathrm{D}^{\mathbb{C}} \varphi_{2} \wedge \ldots \wedge \mathrm{D}^{\mathbb{C}} \varphi_{m}+\ldots \\
& =\sum_{k=1}^{m} \pm \partial^{\prime} \partial^{\prime \prime} \varphi_{k} \wedge \widehat{\omega}_{m-1}\left(\varphi_{1}, \ldots, \widehat{\varphi}_{k}, \ldots, \varphi_{m} ; u\right) .
\end{aligned}
$$

Here the signs are calculated in the standard way using $\left|\mathrm{D}^{\mathbb{C}} \varphi_{i}\right|=\left|\varphi_{i}\right|+1$.

Proof. Denote by $\mathrm{D}_{u}^{\mathbb{C}}$ the differential $\mathrm{D}^{\mathbb{C}}$ restricted to the twistor line $\mathbb{C}$.

Then one has the following simple but fundamental equality:

$$
\begin{aligned}
& \left(\mathbf{d}+\mathrm{d}_{u}\right) \mathrm{D}_{u}^{\mathbb{C}}=\left(\mathbf{d}+\mathrm{d}_{u}\right)\left(u \partial^{\prime}-(1-u) \partial^{\prime \prime}+d u\right)= \\
& \left.d u\left(\partial^{\prime}+\partial^{\prime \prime}\right)+u \partial^{\prime \prime} \partial^{\prime}-(1-u) \partial^{\prime} \partial^{\prime \prime}-d u\left(\partial^{\prime}+\partial^{\prime \prime}\right)\right)=\partial^{\prime \prime} \partial^{\prime} .
\end{aligned}
$$

A conceptual proof of Lemma 2.3. First, we relate the operators $\widehat{\omega}_{m+1}$ and $\omega_{m+1}$.

Let $\Delta$ be the oriented line segment in the twistor plane with the ends $(1,0),(0,1)$ :

$$
\Delta=\{(1-u, u) \mid 0 \leq u \leq 1\} \subset \mathbb{C}^{2} .
$$

Lemma 2.8. The integral of the $\widehat{\omega}_{m+1}$ over the segment $\Delta$ is the operator $(-1)^{m} \omega_{m+1}$ :

$$
\int_{\Delta} \widehat{\omega}_{m+1}\left(\varphi_{0}, \ldots, \varphi_{m} ; z, w\right)=(-1)^{m} \omega_{m+1}\left(\varphi_{0}, \ldots, \varphi_{m}\right) .
$$


Proof. A typical term of $\widehat{\omega}_{m+1}\left(\varphi_{0}, \ldots, \varphi_{m} ; z, w\right)$ containing a differential $z d w-w d z$ is given by

$$
(-1)^{m-k}(z d w-w d z) \wedge \varphi_{0} \wedge w \partial^{\prime} \varphi_{1} \wedge \ldots \wedge w \partial^{\prime} \varphi_{k} \wedge z \partial^{\prime \prime} \varphi_{k+1} \wedge \ldots \wedge z \partial^{\prime \prime} \varphi_{m} .
$$

Let us restrict it to the twistor line and integrate over $0 \leq u \leq 1$. Observe that

$$
\int_{0}^{1}(1-u)^{s} u^{t} d u=\frac{\Gamma(s+1) \Gamma(t+1)}{\Gamma(s+t+2)}=\frac{1}{s+t+1}\left(\begin{array}{c}
s+t \\
s
\end{array}\right)^{-1} .
$$

Therefore after the integration we get

$$
(-1)^{m-k} \frac{k !(m-k) !}{(m+1) !} \varphi_{0} \wedge \partial^{\prime} \varphi_{1} \wedge \ldots \wedge \partial^{\prime} \varphi_{k} \wedge \partial^{\prime \prime} \varphi_{k+1} \wedge \ldots \wedge \partial^{\prime \prime} \varphi_{m} .
$$

Comparing with (56), we get the right hand side of (68).

To prove Lemma 2.3 notice that we integrate in Lemma 2.8 along the segment $\Delta$ on the twistor plane $\mathbb{C}^{2}$, which has boundary. So the integration operator does not quite commute with the de Rham differential on the twistor plane: one gets additional boundary terms provided by the ends. The boundary terms match the two terms in (57), corresponding to the evaluation of the monomials $z^{s} w^{t}$ at $z=1$ when $(s, t)=(m+1,0)$, and at $w=1$ when $(s, t)=(0, m+1)$.

Decomposition of $\widehat{\omega}_{m}$. Since $\Omega_{\mathbb{C}^{2}}^{\leq 1}=\Omega_{\mathbb{C}^{2}}^{0} \oplus \Omega_{\mathbb{C}^{2}}^{1}$, the map $\widehat{\omega}_{m}$ has two components:

$$
\begin{aligned}
& \widehat{\omega}_{m}=\widehat{\eta}_{m}+\widehat{\xi}_{m} . \\
& \widehat{\omega}_{m}^{0}: S^{m}\left(\mathcal{A}^{*, *}[-1]\right) \longrightarrow \mathcal{A}^{*, *} \otimes \Omega_{\mathbb{C}^{2}}^{0} . \\
& \widehat{\omega}_{m}^{1}: S^{m}\left(\mathcal{A}^{*, *}[-1]\right) \longrightarrow \mathcal{A}^{*, *} \otimes \Omega_{\mathbb{C}^{2}}^{1},
\end{aligned}
$$

Explicitly, we have, using the notation ... for the terms obtained by the alternation:

$$
\begin{gathered}
\widehat{\omega}_{m+1}^{1}\left(\varphi_{0}, \ldots, \varphi_{m} ; z, w\right):= \\
\sum_{k=0}^{m}(-1)^{m-k}\left((z d w-w d z) \wedge \varphi_{0} \wedge w \partial^{\prime} \varphi_{1} \wedge \ldots \wedge w \partial^{\prime} \varphi_{k} \wedge z \partial^{\prime \prime} \varphi_{k+1} \wedge \ldots \wedge z \partial^{\prime \prime} \varphi_{m}+\ldots\right) . \\
\widehat{\omega}_{m+1}^{0}\left(\varphi_{0}, \ldots, \varphi_{m} ; z, w\right):=\sum_{k=0}^{m}(-1)^{m-k}\left(w \partial^{\prime} \varphi_{0} \wedge \ldots \wedge w \partial^{\prime} \varphi_{k} \wedge z \partial^{\prime \prime} \varphi_{k+1} \wedge \ldots \wedge z \partial^{\prime \prime} \varphi_{m}+\ldots\right) .
\end{gathered}
$$

\section{Hodge correlators for curves}

\subsection{Green functions on a Riemann surface}

Let $X$ be a smooth compact Riemann surface of genus $g$. There is a canonical hermitian structure on the space $\Omega^{1}(X)$ of holomorphic differentials on $X$ given by

$$
<\alpha_{k}, \alpha_{l}>:=\frac{i}{2} \int_{X(\mathbb{C})} \alpha_{k} \wedge \bar{\alpha}_{l}
$$


Let $\alpha_{1}, . ., \alpha_{g}$ be an orthonormal basis with respect to this form.

Recall that a $p$-current on a manifold is a differential $p$-form with generalised coefficients. Let $\mu$ be a 2-current on $X$ of volume one: $\int_{X} \mu=1$. The corresponding Green current $G_{\mu}(x, y)$ is a 0 -current on $X \times X$ satisfying a differential equation

$$
\bar{\partial} \partial G_{\mu}(x, y)=\delta_{\Delta_{X}}-\left(p_{1}^{*} \mu+p_{2}^{*} \mu-\frac{i}{2} \sum_{k=1}^{g}\left(p_{1}^{*} \alpha_{k} \wedge p_{2}^{*} \bar{\alpha}_{k}+p_{2}^{*} \alpha_{k} \wedge p_{1}^{*} \bar{\alpha}_{k}\right)\right) .
$$

Remark. Our Green function is $(2 \pi i)^{-1}$ times the traditional one, defined using $(2 \pi i)^{-1} \bar{\partial} \partial$ in (174). For example, the Green function for the projective line in our normalisation is

$$
G(x, y)=(2 \pi i)^{-1} \log |x-y| \text {. }
$$

There exists a unique up to a constant solution of this equation. Indeed, the kernel of the Laplacian $\bar{\partial} \partial$ on $X \times X$ consists of constants. One has a symmetry relation:

$$
G_{\mu}(x, y)=G_{\mu}(y, x) .
$$

A point $a \in X$ provides a volume one 2 -current $\delta_{a}$ on $X$. So letting $\mu:=\delta_{a}$, we get a Green function denoted $G_{a}(x, y)$. Then the current on the right hand side of (74) is smooth outside of the divisor $\Delta \cup(\{a\} \times X) \cup(X \times\{a\})$. Since the Laplacian is an elliptic operator, the restriction of the Green current to the complement of this divisor is represented by a smooth function, called the Green function, also denoted by $G_{a}(x, y)$. The Green function $G_{a}(x, y)$ near the singularity divisor looks like $\log r$, where $r$ is a distance to the divisor, plus a smooth function. So it has an integrable singularity, and thus provides a current on $X \times X$, which coincides with the Green current, see [La].

The Arakelov Green function. A classical choice of $\mu$ is given by a smooth volume form of total mass 1 on $X$. In particular a metric on $X$ provides such a volume form. There is a canonical Arakelov volume form on $X$ :

$$
\operatorname{vol}_{X}:=\frac{i}{2 g} \sum_{a=1}^{g} \omega_{a} \wedge \bar{\omega}_{a}, \quad \int_{X} \operatorname{vol}_{X}=1 .
$$

The corresponding Green function is denoted by $G_{\mathrm{Ar}}(x, y)$. It can be normalized so that

$$
p_{2 *}\left(G_{\mathrm{Ar}}(x, y) p_{1}^{*} \operatorname{vol}_{X}\right)=p_{1 *}\left(G_{\mathrm{Ar}}(x, y) p_{2}^{*} \operatorname{vol}_{X}\right)=0 .
$$

The Green function $G_{a}(x, y)$ is expressed via the Arakelov Green function:

$$
G_{a}(x, y)=G_{\operatorname{Ar}}(x, y)-G_{\operatorname{Ar}}(a, y)-G_{\operatorname{Ar}}(x, a)+C .
$$

Indeed, the right hand side satisfies differential equation (744).

A normalization of $G_{a}(x, y)$. Let us choose a non-zero tangent vector $v$ at $a$. Let $t$ be a local parameter at $a$ such that $d t(v)=1$. Then there exists a unique solution $G_{v}(x, y)$ of (74) with $\mu=\delta_{a}$ such that $G_{v}(x, y)-(2 \pi i)^{-1} \log |t|$ vanishes at $x=a$. Indeed, there is a unique normalized Arakelov Green function $G_{\mathrm{Ar}, v}(x, y)$ such that $G_{\mathrm{Ar}, v}(x, a)-(2 \pi i)^{-1} \log |t|$ vanishes at $x=a$. Thanks to the symmetry, the same is true for $G_{\operatorname{Ar}}(a, y)-(2 \pi i)^{-1} \log |t|$ which vanishes at $y=a$. It remains to use (76) with $C=0$ there. 
Specialization. Let $F$ be a function defined in a punctured neighborhood of a point $a$ on a complex curve $X$. Let $v$ be a tangent vector at $a$. Choose a local parameter $t$ in the neighborhood of $a$ such that $d t(v)=1$. Suppose that the function $F(t)$ admits an asymptotic expansion

$$
F(t)=\sum_{n \geq 0} F_{n}(t) \log ^{n}|t|
$$

where $F_{n}(t)$ are smooth functions near $t=0$. We define the specialization $\operatorname{Sp}_{v}^{t \rightarrow 0} F(t):=F_{0}(0)$. We skip sometimes the superscript $t \rightarrow 0$. It follows immediately from the definitions that

$$
\operatorname{Sp}_{v}^{x \rightarrow a} G_{v}(x, y)=\operatorname{Sp}_{v}^{y \rightarrow a} G_{v}(x, y)=0 .
$$

We extend the Green function by linearity to the group of divisors on $X$ by setting $G_{a}\left(\sum n_{i} x_{i}, \sum m_{j} y_{j}\right)=$ $\sum_{i, j} n_{i} m_{j} G_{a}\left(x_{i}, y_{j}\right)$.

Lemma 3.1. If $D_{0}$ is a degree zero divisor, then $G_{\mu}\left(D_{0}, y\right)-G_{\mu^{\prime}}\left(D_{0}, y\right)$ does not depend on $y$.

Proof. One has $\bar{\partial}_{y} \partial_{y}\left(G_{\mu}\left(D_{0}, y\right)-G_{\mu^{\prime}}\left(D_{0}, y\right)\right)=\operatorname{deg}\left(D_{0}\right) p_{2}^{*}\left(\mu-\mu^{\prime}\right)=0$.

\subsection{Construction of Hodge correlators for curves}

Decorated plane trivalent trees. Let us recall some terminology. A tree is a connected graph without loops. The external vertices of a tree are the ones of valency 1 . The rest of the vertices are internal vertices. The edges of a tree consist of external edges, i.e. the ones containing the external vertices, and internal edges. A trivalent tree is a tree whose internal vertices are of valency 3 . We allow a trivalent tree without internal vertices. It is just one edge with two (external) vertices. A trivalent tree with $m+1$ external vertices has $2 m-1$ edges. A plane tree is a tree without self intersections located on the plane.

Definition 3.2. A decoration of a tree $T$ by elements of a set $R$ is a map $\{$ external vertices of $T\} \rightarrow$ $R$.

An example of a decorated plane trivalent tree is given on Figure 3 ,

Recall (Section 1.3) that $S^{*} \subset X$ and

$$
\mathrm{V}_{X, S^{*}}^{\vee}=\mathbb{C}\left[S^{*}\right] \oplus\left(\Omega_{X}^{1} \oplus \bar{\Omega}_{X}^{1}\right) .
$$

Further, $\mathcal{C}_{X, S^{*}}^{\vee}$ is the cyclic envelope of the tensor algebra of $\mathrm{V}_{X, S^{*}}^{\vee}$. Its elements are cyclic words in $\mathrm{V}_{X, S^{*}}^{\vee}$ : for instance, one has $\mathcal{C}(a b c)=\mathcal{C}(c a b)$.

A 2-current $\mu$ is admissible if it is either a smooth volume form of total mass 1 on $X$, or the $\delta$-current $\delta_{a}$ for some point $a \in X$. Given an admissible 2-current $\mu$, we are going to define a linear map, the Hodge correlator map:

$$
\operatorname{Cor}_{\mathcal{H}, \mu}: \mathcal{C}_{X, S^{*}}^{\vee} \longrightarrow \mathbb{C}
$$

To define it, take a degree $m+1$ decomposable cyclic element

$$
W=\mathcal{C}\left(v_{0} \otimes v_{1} \otimes \ldots \otimes v_{m}\right), \quad v_{i} \in \mathrm{V}_{X, S^{*}}^{\vee}
$$


The external vertices of a plane tree have a cyclic order provided by a chosen (say, clockwise) orientation of the plane. We say that a plane trivalent tree $T$ is decorated by $W$ if the external vertices of the tree are decorated by the elements $v_{0}, \ldots, v_{m}$, respecting the cyclic order of the vertices, as on Figure 3. Although the decoration depends on the presentation of $W$ as a cyclic tensor product of the vectors $v_{i}$, constructions below depend only on $W$ and not on such presentation. We are going to assign to $W$ a $2 m$-current $\kappa_{W}$ on

$$
X^{\{\text {vertices of } T\}} \text {. }
$$

We assume that each of the vectors $v_{i}$ in $W$ is either a generator $\left\{s_{i}\right\} \in \mathbb{C}\left[S^{*}\right]$, or a 1 -form $\omega$

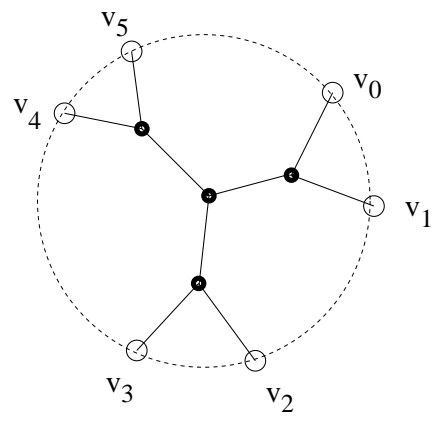

Figure 3: A decorated plane trivalent tree.

on $X$. In Section 2.2 we assume in addition that if $T$ has just one edge, both its vertices are $S$-decorated. Then a decoration of $T$ provides a decomposition of the set of external edges into the $S^{*}$-decorated and special edges: the latter are decorated by 1-forms, the former by points of $S^{*}$.

Denote by $\delta_{X}$ the $\delta$-current of the diagonal in $X \times X$, and by $\delta_{X} \omega$ its product by a smooth form $p^{*} \omega$, where $\omega$ is a form on $X$ and $p: X \times X \rightarrow X$ is the projection to one of the factors. Given an edge $E$ of $T$, we assign to it a current $\widetilde{G}_{E}$ on $X \times X$ :

$$
\widetilde{G}_{E}:= \begin{cases}\delta_{X} \omega & \text { if } E \text { is a special external edge } \\ G_{\mu}\left(x_{1}, x_{2}\right) & \text { otherwise. }\end{cases}
$$

For each edge $E$ of $T$, there is a projection of the set of vertices of $T$ onto the set of vertices of $E$. It gives rise to a canonical projection

$$
p_{E}: X^{\{\text {vertices of } T\}} \rightarrow X^{\{\text {vertices of } \mathrm{E}\}}=X \times X \text {. }
$$

We set

$$
G_{E}:=p_{E}^{*} \widetilde{G}_{E}
$$

Since the map $p_{E}$ is transversal to the wave front of the current $\widetilde{G}_{E}$, the inverse image is well defined.

Given a finite set $\mathcal{X}$, there is a $\mathbb{Z} / 2 \mathbb{Z}$-torsor or $\mathcal{X}$, called the orientation torsor of $\mathcal{X}$. Its elements are expressions $x_{1} \wedge \ldots \wedge x_{|\mathcal{X}|}$, where $\left\{x_{1}, \ldots, x_{|\mathcal{X}|}\right\}=\mathcal{X}$; interchanging two neighbors we change the sign of the expression. The orientation torsor or $_{T}$ of a graph $T$ is the orientation torsor of the set of edges of $T$. An orientation of the plane induces an orientation of a plane 
trivalent tree: Indeed, the orientation of the plane provides orientations of links of each of the vertices.

Let us choose an element

$$
\left(E_{0} \wedge \ldots \wedge E_{r}\right) \wedge\left(E_{r+1} \wedge \ldots \wedge E_{2 m}\right) \in \text { or }_{T}
$$

of the orientation torsor of our tree $T$, such that the edges $E_{0}, \ldots, E_{r}$ are internal or $S$-decorated, and the others are decorated by 1 -forms. Set

$$
\kappa_{T}(W):=\operatorname{sgn}\left(E_{0} \wedge \ldots \wedge E_{2 m}\right) \omega_{r}\left(G_{E_{0}} \wedge \ldots \wedge G_{E_{r}}\right) \wedge G_{E_{r+1}} \wedge \ldots \wedge G_{E_{2 m}},
$$

where $\operatorname{sign}\left(E_{0} \wedge \ldots \wedge E_{2 m}\right) \in\{ \pm 1\}$ is the difference between the element $E_{0} \wedge \ldots \wedge E_{2 m}$ and the canonical generator of or $_{T}$.

Lemma 3.3. $\kappa_{T}(W)$ is a $2 m$-current on 78.).

Proof. Consider the smooth differential $r$-form

$$
\omega_{r}\left(G_{E_{0}} \wedge \ldots \wedge G_{E_{r}}\right)
$$

on the complement to the diagonals in (78). We claim that it has integrable singularities, i.e. provides an $r$-current on (78). Indeed, the Green function is smooth outside of the diagonal. Its singularity near the diagonal is of type $\log |t|$, where $t$ is a local equation of the diagonal i.e. $G(x, y)-\log |t|$ is smooth near the diagonal. Since $\omega_{m+1}$ is a linear map, it is sufficient to prove the claim for a specific collection of functions with such singularities. Since the problem is local, we can choose local equations $f_{i}=0$ of all the diagonals involved, and consider the functions $\log \left|f_{i}\right|$ as such a specific collection. Then the claim follows from Theorem 2.4 in [G8].

Consider the projection map

$$
p_{T}: X^{\{\text {vertices of } \mathrm{T}\}} \longrightarrow X^{\left\{S^{*} \text {-decorated vertices of } \mathrm{T}\right\}} .
$$

Definition 3.4. Let $\mu$ be an admissible 2-current on $X$, and $W \in \mathcal{C}_{X, S^{*}}^{\vee}$. Then the correlator $\operatorname{Cor}_{\mathcal{H}}(W)$ is a 0 -current on the right hand side of (82), given by

$$
\operatorname{Cor}_{\mathcal{H}}(W):=p_{T *}\left(\sum_{T} \kappa_{T}(W)\right),
$$

where the sum is over all plane trivalent trees $T$ decorated by the cyclic word $W$.

The restriction of the current $\operatorname{Cor}_{\mathcal{H}}(W)$ to the complement of the diagonals is a smooth function.

Remarks. 1. The direct image $p_{T *}$ is given by integration over $X^{\{\text {internal vertices of } T\}}$. Indeed, $\kappa_{T}(W)$ is the exterior product of (81) and a smooth differential form on the product of copies of $X$ corresponding to the internal and $S^{*}$-decorated vertices, extended by the $\delta$-function to $(78)$.

2. We may assume that the $S$-decorated vertices are decorated by divisors supported on $S$ by extending the Hodge correlator map by linearity.

3. For a given measure $\mu$, the corresponding Green function $G_{\mu}$ is defined up to adding a constant. The Hodge correlators depend on it. However this dependence is minimal. If $W$ has at least one degree zero divisor $D$ as a factor, the corresponding Hodge correlator does not depend on the choice of the constant. Indeed, we may assume that in the form $\omega_{m+1}$ we do not differentiate the Green function $G_{E}$ assigned to the edge $E$ decorated by $D$. 


\subsection{Shuffle relations for Hodge correlators}

Our goal is the following shuffle relations for Hodge correlators:

Proposition 3.5. Let $v_{i} \in \mathrm{V}_{X, S^{*}}^{\vee}$. Then for any $p, q \geq 1$ one has

$$
\sum_{\sigma \in \Sigma_{p, q}} \operatorname{Cor}_{\mathcal{H}} \mathcal{C}\left(v_{0} \otimes v_{\sigma(1)} \otimes \ldots \otimes v_{\sigma(p+q)}\right)=0
$$

where the sum is over all $(p, q)$-shuffles $\sigma \in \Sigma_{p, q}$.

To prove this we interpret the "sum over plane trivalent trees" construction using cyclic operads. See [LV] for the background on operads.

Trees and the cyclic Lie co-operad. Let $\mathcal{C} \mathcal{A} s s_{\bullet}$ be the cyclic associative operad. This means, in particular, the following. Let $\mathcal{A} s s_{m}\left\{v_{1}, \ldots, v_{m}\right\}$ be the space of all associative words formed by the letters $v_{1}, \ldots, v_{m}$, each used once. Then $\mathcal{C} \mathcal{A} s s_{m+1}$ is a vector space generated by the expressions

$$
\left(A, v_{0}\right), \quad \text { where } A \in \mathcal{A} s s_{m}\left\{v_{1}, \ldots, v_{m}\right\} ; \quad(x y, z)=(x, y z), \quad(x, y)=(y, x),
$$

i.e. $(*, *)$ is an invariant scalar product on an associative algebra. So as a vector space $\mathcal{C} \mathcal{A} s s_{m+1}$ is isomorphic to the space of $m$-ary operations $\mathcal{A} s s_{m}$ in the associative operad, or simply speaking to $\mathcal{A} s s_{m}\left\{v_{1}, \ldots, v_{m}\right\}$.

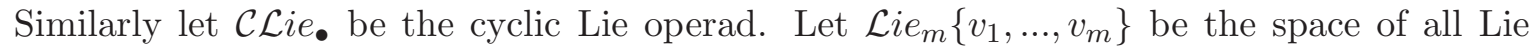
words formed by the letters $v_{1}, \ldots, v_{m}$, each used once. Then as a vector space $\mathcal{C} \mathcal{L} i e_{m+1}$ is isomorphic to the space of $m$-ary operations in the Lie operad. We think about it as of the space generated by expressions

$$
\left(L, v_{0}\right), \quad \text { where } L \in \mathcal{L}_{i e}\left\{v_{1}, \ldots, v_{m}\right\} ; \quad([x, y], z)=(x,[y, z]), \quad(x, y)=(y, x),
$$

i.e. $(*, *)$ is an invariant scalar product on a Lie algebra.

Example. The space $\mathcal{C} \mathcal{L} i e_{3}$ is one dimensional. It is generated by $\left(\left[v_{1}, v_{2}\right], v_{0}\right)$. The space $\mathcal{C} \mathcal{A} s s_{3}$ is two dimensional, generated by $\left(v_{1} v_{2}, v_{0}\right)$ and $\left(v_{2} v_{1}, v_{0}\right)$.

Let $\mathcal{C} \mathcal{A} s s_{m+1}^{*}\left(\right.$ resp. $\left.\mathcal{C} \mathcal{L} i e_{m+1}^{*}\right)$ be the $\mathbb{Q}$-vector space dual to $\mathcal{C} \mathcal{A} s s_{m+1}$ (resp. $\left.\mathcal{C} \mathcal{L} i e_{m+1}\right)$. We define the subspace of shuffle relations in $\mathcal{C} \mathcal{A} s s_{m+1}^{*}$ as follows. Let $\left(v_{0} v_{1} \ldots v_{m}\right)^{*} \in \mathcal{C} \mathcal{A} s s_{m+1}^{*}$ be a functional whose value on $\left(v_{1} \ldots v_{m}, v_{0}\right)$ is 1 and on $\left(v_{i_{1}} \ldots v_{i_{m}}, v_{0}\right)$ is zero if $\left\{i_{1}, \ldots, i_{m}\right\} \neq$ $\{1,2, \ldots, m\}$ as ordered sets. The subspace of shuffle relations is generated by the expressions

$$
\sum_{\sigma \in \Sigma_{k, m-k}}\left(v_{0} v_{\sigma(1)} \ldots v_{\sigma(m)}\right)^{*} ; \quad 1 \leq k \leq m-1 .
$$

Lemma 3.6. There is a canonical isomorphism

$$
\mathcal{C} \mathcal{L} i e_{m+1}^{*}=\frac{\mathcal{C} \mathcal{A} s s_{m+1}^{*}}{\text { Shuffle relations }} .
$$

Proof. Notice that $\mathcal{L i e}_{m}\left\{v_{1}, \ldots, v_{m}\right\} \subset \mathcal{A} s s_{m}\left\{v_{1}, \ldots, v_{m}\right\}$ is the subspace of primitive elements for the coproduct $\Delta, \Delta\left(v_{i}\right)=v_{i} \otimes 1+1 \otimes v_{i}$. Thus its dual $\mathcal{L} i e_{m}^{*}\left\{v_{1}, \ldots, v_{m}\right\}$ is the quotient of $\mathcal{A} s s_{m}^{*}\left\{v_{1}, \ldots, v_{m}\right\}$ by the shuffle relations, see [LV], Theorem 1.3.9. 
The tree complex $([\underline{K}])$. Denote by $\mathrm{T}_{(m)}^{i}$ the abelian group generated by the isomorphism classes of pairs $\left(T, \mathrm{Or}_{T}\right)$ where $T$ is a tree (not a plane tree!) with $2 m-i$ edges and with $m+1$ ends decorated by the set $\left\{v_{0}, \ldots, v_{m}\right\}$. Here $\operatorname{Or}_{T}$ is an orientation of the tree $T$. The only relation is that changing the orientation of a tree $T$ amounts to changing the sign of the generator. There is a differential $d: \mathrm{T}_{(m)}^{i} \longrightarrow \mathrm{T}_{(m)}^{i+1}$ defined by shrinking internal edges of a tree:

$$
\left(T, \mathrm{Or}_{T}\right) \longmapsto \sum_{\text {internal edges } E \text { of } T}\left(T / E, \mathrm{Or}_{T / E}\right) .
$$

Here if $\mathrm{Or}_{T}=E \wedge E_{1} \wedge \ldots$, then $\mathrm{Or}_{T / E}:=E_{1} \wedge \ldots$.

The group $\mathrm{T}_{(m)}^{i}$ is a free abelian group with a basis; the basis vectors are defined up to a sign. Thus there is a perfect pairing $\mathrm{T}_{(m)}^{i} \otimes \mathrm{T}_{(m)}^{i} \longrightarrow \mathbb{Z}$, and we may identify the group $\mathrm{T}_{(m)}^{i}$ with its dual $\left(\mathrm{T}_{(m)}^{i}\right)^{*}:=\operatorname{Hom}\left(\mathrm{T}_{(m)}^{i}, \mathbb{Z}\right)$. Consider the map $w$

$$
\left(\mathrm{T}_{(m)}^{1}\right)^{*} \quad \stackrel{w}{\longrightarrow} \mathcal{C} \mathcal{L} i e_{m+1} \quad \stackrel{i}{\hookrightarrow} \mathcal{C} \mathcal{A} s s_{m+1} .
$$

defined as follows. Take an element $\left(T, \mathrm{Or}_{T}\right)$ represented by a 3 -valent tree $T$ decorated by $v_{0}, v_{1}, \ldots, v_{m}$ and an orientation $\mathrm{Or}_{T}$ of $T$. Make a decorated tree rooted at $v_{0}$ out of the tree $T$. Define a Lie word in $v_{1}, \ldots, v_{m}$ using the oriented tree. We are getting an element $w(T) \subset \mathcal{L} \mathcal{L} i e_{m+1}$. Viewing it as an associative word, we arrive at the element $i \circ w(T)$. There are the dual maps

$$
\mathcal{C} \mathcal{A} s s_{m+1}^{*} \stackrel{i^{*}}{\longrightarrow} \mathcal{C} \mathcal{L} i e_{m+1}^{*} \quad \stackrel{w^{*}}{\longrightarrow} \quad \mathrm{T}_{(m)}^{1} .
$$

The next lemma offers an explanation of the "sum over plane trivalent trees" construction.

Lemma 3.7. The element $w^{*} \circ i^{*}\left(v_{0} v_{1} \ldots v_{m}\right)^{*}$ is the sum of all plane trivalent trees decorated by the cyclic word $\mathcal{C}\left(v_{0} v_{1} \ldots v_{m}\right)$, and equipped with the canonical orientation induced by the clockwise orientation of the plane.

For any $p, q \geq 1$ with $p+q=m$ one has

$$
w^{*} \circ i^{*} \sum_{\sigma \in \Sigma_{p, q}}\left(v_{0} v_{\sigma(1)} \ldots v_{\sigma(m)}\right)^{*}=0
$$

where the sum is over all $(p, q)$-shuffles. Indeed, we apply $w^{*}$ to the expression which is zero by Lemma 3.6. This plus Lemma 3.7 immediately imply

Corollary 3.8. The element of $\mathrm{T}_{(m)}^{1}$ given by the sum over all shuffles $\sigma \in \Sigma_{p, q}$ of the sum of all plane trivalent trees decorated by $\left(v_{0} v_{\sigma(1)} \ldots v_{\sigma(m)}\right)$ is zero.

Proof of Proposition 3.5. Corollary 3.8 tells that the integral in (83) is over zero cycle.

Remark. It was proved in $[\mathrm{GK}]$ that the following complex is exact:

$$
\mathcal{C} \mathcal{L} i e_{m+1}^{*} \quad \stackrel{w^{*}}{\longrightarrow} \quad \mathrm{T}_{(m)}^{1} \stackrel{d}{\longrightarrow} \mathrm{T}_{(m)}^{2} \stackrel{d}{\longrightarrow} \ldots \stackrel{d}{\longrightarrow} \mathrm{T}_{(m)}^{m-2} .
$$




\section{The twistor transform and the Hodge complex functor}

\subsection{The Dolbeaut complex of a variation of Hodge structures.}

Variations of Hodge structures. Let $X$ be a complex manifold, given by a $C^{\infty}$-manifold $\mathcal{X}$ with an integrable almost complex structure $I$. Then $\bar{X}:=(\mathcal{X},-I)$ is the complex conjugate manifold. Denote by $\mathcal{A}_{X}^{p, q}$ the space of smooth $(p, q)$-forms on $X$. So $\mathcal{A}_{\bar{X}}^{p, q}=\mathcal{A}_{X}^{q, p}$.

Given a complex $C^{\infty}$-vector bundle $\mathcal{E}$ on $\mathcal{X}$, a structure of a holomorphic vector bundle on $\mathcal{E}$ over the complex manifold $X$ is determined by an integrable $\bar{\partial}$-connection on $\mathcal{E}$ :

$$
\bar{\partial}: \mathcal{E} \longrightarrow \mathcal{E} \otimes \mathcal{A}_{X}^{0,1}, \quad \bar{\partial}^{2}=0
$$

Denote by $E$ the holomorphic vector bundle on $X$ determined by the $\bar{\partial}$-connection. Its sheaf of holomorphic sections is given by the sections of $\mathcal{E}$ annihilated by $\bar{\partial}$.

A filtration $F^{\bullet}$ by holomorphic subbundles of $E$ can be described by a decreasing filtration $F^{p} \mathcal{E} \supset F^{p+1} \mathcal{E} \supset \ldots$ by $C^{\infty}$-subbundles, preserved by $\bar{\partial}$ :

$$
\bar{\partial}: F^{p} \mathcal{E} \longrightarrow F^{p} \mathcal{E} \otimes \mathcal{A}_{X}^{0,1}
$$

Let $(\mathcal{E}, \mathbf{d})$ be a complex $C^{\infty}$-vector bundle $\mathcal{E}$ with a connection $\mathbf{d}$ on a manifold $\mathcal{X}$.

If $\mathcal{X}$ has a structure of a complex manifold $X$, the connection has a decomposition

$$
\mathbf{d}=\partial_{X}+\bar{\partial}_{X}, \quad \partial_{X}: \mathcal{E} \longrightarrow \mathcal{E} \otimes \mathcal{A}_{X}^{1,0}, \quad \bar{\partial}_{X}: \mathcal{E} \longrightarrow \mathcal{E} \otimes \mathcal{A}_{X}^{0,1}
$$

The same connection, considered on the complex conjugate manifold $\bar{X}$, has a decomposition

$$
\mathbf{d}=\partial_{\bar{X}}+\bar{\partial}_{\bar{X}}, \quad \partial_{\bar{X}}=\bar{\partial}_{X}, \quad \bar{\partial}_{\bar{X}}=\partial_{X} .
$$

A $C^{\infty}$-filtration $F^{\bullet}$ of $\mathcal{E}$ and a connection $\mathbf{d}$ on $\mathcal{E}$ satisfy the Griffiths transversality condition on a complex manifold $X=(\mathcal{X}, I)$ if

$$
\partial_{X}: F^{p} \mathcal{E} \longrightarrow F^{p-1} \mathcal{E} \otimes \mathcal{A}_{X}^{1,0}
$$

So a filtered $C^{\infty}$-vector bundle with connection $\left(\mathcal{E}, F^{\bullet}, \mathbf{d}\right)$ gives rise to a holomorphic vector bundle $E$ on $X$ with a filtration by holomorphic subbundles satisfying the Griffiths transversality condition if and only if

$$
\bar{\partial}_{X}^{2}=0, \quad \bar{\partial}_{X}: F^{p} \mathcal{E} \longrightarrow F^{p} \mathcal{E} \otimes \mathcal{A}_{X}^{0,1}, \quad \partial_{X}: F^{p} \mathcal{E} \longrightarrow F^{p-1} \mathcal{E} \otimes \mathcal{A}_{X}^{1,0} .
$$

So it makes sense to combine the two conditions on the triple $\left(\mathcal{E}, F^{\bullet}, \mathbf{d}\right)$ into a single one, which we call the Griffiths condition.

Definition 4.1. A filtered $C^{\infty}$-vector bundle with connection $\left(\mathcal{E}, F^{\bullet}, \mathbf{d}\right)$ on a complex manifold $X$ satisfies the Griffiths condition if it satisfies 87).

Recall that two filtration $F^{\bullet}$ and $\bar{F}^{\bullet}$ on a complex vector space $V$ are $n$-complimentary if

$$
\operatorname{gr}_{F}^{p} \operatorname{gr}_{F}^{q} V=0 \text { unless } p+q=n \text {. }
$$

In this case the vector space $V$ is bigraded of pure weight $n$ :

$$
V=\oplus_{p+q=n} \operatorname{gr}_{F}^{p} \operatorname{gr}_{F}^{q} V
$$

Given a complex vector space $V$, denote by $\bar{V}$ the complex conjugate vector space. It coincides with $V$ as a real vector space. The new action $\circ$ of $\mathbb{C}$ given by $\lambda \circ v:=\bar{\lambda} v$. 
Definition 4.2. A weight $n$ variation of Hodge structure $\unlhd^{4}$ is a data $\left(X, \mathcal{E}, \mathbf{d} ; F^{\bullet}, \bar{F}^{\bullet}\right)$, given by:

- A complex manifold $X$; a complex $C^{\infty}$-vector bundle $\mathcal{E}$ with a flat connection $\mathbf{d}$ on $X$.

- A filtration $F^{\bullet}$ by subbundles of $\mathcal{E}$ satisfying the Griffiths condition;

$$
\bar{\partial}_{X}: F^{p} \mathcal{E} \longrightarrow F^{p} \mathcal{E} \otimes \mathcal{A}_{X}^{0,1}, \quad \partial_{X}: F^{p} \mathcal{E} \longrightarrow F^{p-1} \mathcal{E} \otimes \mathcal{A}_{X}^{1,0} .
$$

- A filtration $\bar{F}^{\bullet}$ by subbundles of $\overline{\mathcal{E}}$ satisfying the Griffiths condition:

$$
\partial_{X}: \bar{F}^{p} \overline{\mathcal{E}} \longrightarrow \bar{F}^{p} \overline{\mathcal{E}} \otimes \mathcal{A}_{X}^{1,0}, \quad \bar{\partial}_{X}: \bar{F}^{p} \overline{\mathcal{E}} \longrightarrow \bar{F}^{p-1} \overline{\mathcal{E}} \otimes \mathcal{A}_{X}^{0,1}
$$

- Filtrations $F^{\bullet}$ and $\bar{F}^{\bullet}$ are n-complimentary.

A variation of Hodge structures is a direct sum of variations of different weights.

Denote by $\overline{\mathcal{E}}$ the complex conjugate vector bundle to $\mathcal{E}$. If $E$ is a holomorphic vector bundle over a complex manifold $X$, then $\bar{E}$ is a holomorphic vector bundle over the $\bar{X}$. The conditions on $\bar{F}^{\bullet}$ just mean that it is a filtration of the complex bundle $\overline{\mathcal{E}}$ on $\bar{X}$ by subbundles satisfying the Griffiths condition.

The Galois group $\operatorname{Gal}(\mathbb{C} / \mathbb{R})$ acts on the category of variations of complex Hodge structures. Its generator acts by

$$
\left(X, \mathcal{E}, \mathbf{d} ; F^{\bullet}, \bar{F}^{\bullet}\right) \longrightarrow\left(\bar{X}, \overline{\mathcal{E}}, \mathbf{d} ; \bar{F}^{\bullet}, F^{\bullet}\right) .
$$

Definition 4.3. The category $\mathbb{R}-M H S$ of real variations of Hodge structures is the category of $\operatorname{Gal}(\mathbb{C} / \mathbb{R})$-equivariant objects in the category $M H S$.

Equivalently, using the traditional terminology, a real weight $n$ variation of Hodge structures is given by a real local system $\mathcal{L}$ on a complex manifold $X$, whose complexification is equipped with a filtration $F^{\bullet}$ by holomorphic subbundles satisfying Griffiths transversality condition. Finally, the filtration $F^{\bullet}$ and its complex conjugate $\bar{F}^{\bullet}$ are $n$-complementary.

The Dolbeaut complex of a variation of Hodge structures. Let $(\mathcal{L}, \mathbf{d})$ be a variation of Hodge structures over $X$. Here $\mathcal{L}$ denotes the underlying complex local system. Its de Rham complex $\mathcal{A}^{*}(\mathcal{L}):=\mathcal{A}^{*} \otimes_{\mathbb{C}} \mathcal{L}$ has a differential, also denoted by $\mathrm{d}$. Recall the Hodge decomposition

$$
\mathcal{L}=\oplus_{p, q} \mathcal{L}^{p, q}
$$

We define the Dolbeaut bicomplex of $\mathcal{L}$ by setting

$$
\mathcal{A}^{*, *}(\mathcal{L})=\oplus_{s, t} \mathcal{A}^{s, t}(\mathcal{L}), \quad \mathcal{A}^{s, t}(\mathcal{L}):=\oplus_{s=a+p, t=b+q} \mathcal{A}^{a, b} \otimes \mathbb{C} \mathcal{L}^{p, q}
$$

It is bigraded by the Hodge bidegree $(s, t)$, and equipped with the differential $\mathbf{d}$.

Now we use the fact that $\mathcal{L}$ is a variation of Hodge structures. The Griffiths conditions for $F^{\bullet}$ and $\bar{F}^{\bullet}$ just mean that the components of the differential $\mathbf{d}$ are of Hodge bidegrees $(1,0)$ and $(0,1)$.

\footnotetext{
${ }^{4}$ What we call a variation of Hodge structures is usually called a variation of complex Hodge structures.
} 
Let $\mathbf{d}=\partial^{\prime}+\partial^{\prime \prime}$ be the decomposition into components of Hodge bidegrees $(1,0)$ and $(0,1)$. Decomposing $\mathbf{d}^{2}=0$ into the components of Hodge bidegrees $(2,0),(1,1)$ and $(0,2)$ we get

$$
\partial^{\prime 2}=\partial^{\prime \prime 2}=\partial^{\prime} \partial^{\prime \prime}+\partial^{\prime \prime} \partial^{\prime}=0 .
$$

So we get the Dolbeaut bicomplex $\left(\mathcal{A}^{*, *}(\mathcal{L}), \partial^{\prime}, \partial^{\prime \prime}\right)$ of a variation of Hodge structures. It satisfies the $\partial \bar{\partial}$-lemma. Its total complex is the Rham complex. Set

$$
\mathbf{d}^{\mathbb{C}}:=\partial^{\prime}-\partial^{\prime \prime} .
$$

Therefore the $\left(\mathcal{A}^{*, *}(\mathcal{L}), \partial^{\prime}, \partial^{\prime \prime}\right)$ is a cohomological Dolbeaut complex defined in Section 2 .

Remark. Let $\mathbf{d}=\partial+\bar{\partial}$ be the decomposition into the holomorphic and antiholomorphic components. Let $\partial_{0}$ and $\bar{\partial}_{0}$ be the components of $\partial$ and $\bar{\partial}$ preserving the Hodge decomposition. Then we have

$$
\begin{gathered}
\partial=\partial_{0}+\lambda^{1,0}, \quad \lambda^{1,0} \in \operatorname{Hom}\left(\mathcal{L}^{*, *}, \mathcal{L}^{*-1, *+1}\right) \otimes \mathcal{A}^{1,0}, \\
\bar{\partial}=\bar{\partial}_{0}+\lambda^{0,1}, \quad \lambda^{0,1} \in \operatorname{Hom}\left(\mathcal{L}^{*, *}, \mathcal{L}^{*+1, *-1}\right) \otimes \mathcal{A}^{0,1} . \\
\partial^{\prime}=\partial_{0}+\lambda^{0,1}, \quad \partial^{\prime \prime}=\bar{\partial}_{0}+\lambda^{1,0}
\end{gathered}
$$

However we avoid using these formulas and operators $\partial$ and $\bar{\partial}$.

\subsection{The Hodge complex functor $\mathcal{C}_{\mathcal{H}}^{\bullet}(-)$}

In Section 4.2 we define the Hodge complex $\mathcal{C}_{\mathcal{H}}^{\bullet}\left(\mathcal{A}^{*, *}\right)$ of a cohomological Dolbeaut complex $\mathcal{A}^{*, *}$. Using the natural structure of a Hodge-Deligne complex on a cohomological Dolbeaut complex $\mathcal{A}^{*, *}$, we define a quasiisomorphism

$$
\operatorname{RHom}_{\mathrm{MHS}}\left(\mathbb{C}(0), \mathcal{A}^{*, *}\right) \stackrel{\text { qis }}{\longrightarrow} \mathcal{C}_{\mathcal{H}}^{\bullet}\left(\mathcal{A}^{*, *}\right) .
$$

Therefore the Hodge complex $\mathcal{C}_{\mathcal{H}}(\mathcal{L})$ of a variation of Hodge structures $\mathcal{L}$ on a complex manifold $M$ calculates the Hodge cohomology of $\mathcal{L}$. There are similar results for the $\mathbb{R}$-Hodge complex.

The Hodge complex $\mathcal{C}_{\mathcal{H}}^{\bullet}\left(\mathcal{A}^{*, *}\right)$. Let $\mathcal{A}^{*, *}$ be a cohomological Dolbeaut complex, bigraded by the Hodge bidegree. Its cohomological grading is given by the cohomological degree $\operatorname{deg}_{\mathcal{A}}$. The differential is a sum $\mathbf{d}=\partial^{\prime}+\partial^{\prime \prime}$ of two anticommuting differentials, where $\partial^{\prime}$ is of Hodge bidegree $(1,0)$, and $\partial^{\prime \prime}$ is of Hodge bidegree $(0,1)$.

Let $\mathcal{A}_{\mathrm{cl}}^{0,0}$ be the subspace of $\mathbf{d}$-closed elements in $\mathcal{A}^{0,0}$. Consider the following subspace of $\mathcal{A}^{*, *}$ sitting in non-positive Hodge bidegrees, see Fig 4 ;

$$
\mathcal{C}_{\mathcal{H}}\left(\mathcal{A}^{*, *}\right):=\bigoplus_{s, t \leq-1} \mathcal{A}^{s, t} \bigoplus \mathcal{A}_{\mathrm{cl}}^{0,0}
$$

Let us define a degree $\operatorname{deg}$ on $\mathcal{C}_{\mathcal{H}}\left(\mathcal{A}^{*, *}\right)$. Given an element $\varphi$ of $\operatorname{degree} \operatorname{deg}_{\mathcal{A}}(\varphi)$ in $\mathcal{A}^{s, t}$, set

$$
\operatorname{deg}(\varphi):= \begin{cases}\operatorname{deg}_{\mathcal{A}}(\varphi) & \text { if }(s, t)=(0,0) \\ \operatorname{deg}_{\mathcal{A}}(\varphi)+1 & \text { otherwise. }\end{cases}
$$




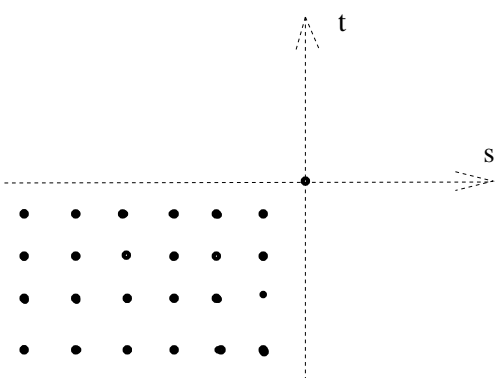

Figure 4: The Hodge bigrading $(s, t)$ of the Hodge complex $\mathcal{C}_{\mathcal{H}}^{\bullet}\left(\mathcal{A}^{*, *}\right)$.

In other words,

$$
\mathcal{C}_{\mathcal{H}}^{\bullet}\left(\mathcal{A}^{*, *}\right):=\bigoplus_{s, t \leq-1}\left(\mathcal{A}^{s, t}\right)^{\bullet}[-1] \bigoplus\left(\mathcal{A}_{\mathrm{cl}}^{0,0}\right)^{\bullet}
$$

We define a degree 1 differential $D$ on $\mathcal{C}_{\mathcal{H}}^{\bullet}\left(\mathcal{A}^{*, *}\right)$ by setting

$$
D \varphi= \begin{cases}-\frac{1}{2} \mathbf{d d}^{\mathbb{C}} & \text { if }(s, t)=(-1,-1), \\ \partial^{\prime} \varphi & \text { if } s<-1, t=-1, \\ \partial^{\prime \prime} \varphi & \text { if } s=-1, t<-1, \\ \mathbf{d} \varphi & \text { otherwise }\end{cases}
$$

So unless $(s, t)=(-1,-1)$, the map $D$ is the differential $\mathbf{d}$ truncated to fit the rectangle $s, t \leq-1$.

Conventions. Below $\varphi_{s, t}$ denotes a homogeneous element of the Hodge bidegree $(-s,-t)$ in the Hodge complex, so $s, t \geq 0$. Abusing notation, we still denote by $\partial^{\prime}$ and $\partial^{\prime \prime}$ the components of $D$ of the Hodge bidegrees $(1,0)$ and $(0,1)$. So in the Hodge complex $\partial^{\prime} \varphi_{1, t}=0$ and $\partial^{\prime \prime} \varphi_{s, 1}=0$, while this may not be so in the original bicomplex $\mathcal{A}^{*, *}$. We either omit the cohomological grading, or denote it by $\bullet$. The Hodge bidegree is denoted by $(*, *)$.

Let $N$ be a linear operator on $\mathcal{C}_{\mathcal{H}}^{\bullet}\left(\mathcal{A}^{*, *}\right)$ acting on a homogeneous element $\varphi_{s, t}$ by

$$
N \varphi_{s, t}= \begin{cases}0 & \text { if }(s, t)=(0,0) \\ (s+t-1) \varphi_{s, t} & \text { otherwise }\end{cases}
$$

We denote by $N_{\varphi}$ the eigenvalue of the operator $N$ on a homogeneous element $\varphi$.

The real Hodge complex functor $\mathcal{C}_{\mathcal{H}, \mathbb{R}}(-)$. Let $\mathcal{A}_{\mathbb{R}}^{*}$ be a real graded vector space with a degree +1 cohomological differential $\mathbf{d}$, whose complexification $\mathcal{A}_{\mathbb{R}}^{*} \otimes_{\mathbb{R}} \mathbb{C}$ is a cohomological Dolbeaut bicomplex equipped with an antiholomorphic involution $\varphi \longmapsto \bar{\varphi}$ :

$$
\mathcal{A}_{\mathbb{R}}^{n} \otimes_{\mathbb{R}} \mathbb{C}=\oplus_{s+t=n} \mathcal{A}^{s, t}, \quad \overline{\mathcal{A}^{s, t}}=\mathcal{A}^{t, s} .
$$

We define the real Hodge complex $\mathcal{C}_{\mathcal{H} ; \mathbb{R}}^{\bullet}\left(\mathcal{A}_{\mathbb{R}}^{*}\right)$ as a subcomplex

$$
\mathcal{C}_{\mathcal{H} ; \mathbb{R}}^{\bullet}\left(\mathcal{A}_{\mathbb{R}}^{*}\right) \subset \mathcal{C}_{\mathcal{H}}^{\bullet}\left(\mathcal{A}^{*, *}\right) .
$$

Namely, a homogeneous element $\varphi \in \mathcal{C}_{\mathcal{H}}^{\bullet}\left(\mathcal{A}^{*, *}\right)$ is in $\mathcal{C}_{\mathcal{H} ; \mathbb{R}}^{\bullet}\left(\mathcal{A}_{\mathbb{R}}^{*}\right)$ if and only if

$$
\bar{\varphi}= \begin{cases}\varphi & \text { if }(s, t)=(0,0) . \\ -\varphi & \text { otherwise }\end{cases}
$$


The Hodge complex $\mathcal{C}_{\mathcal{H}}^{\bullet}(\mathcal{L})$. Applying the functor $\mathcal{C}_{\mathcal{H}}(-)$ to the cohomological Dolbeaut bicomplex $\mathcal{A}^{*, *}(\mathcal{L})$ provided by a variation of Hodge structures $\mathcal{L}$, we get the Hodge complex $\mathcal{C}_{\mathcal{H}}^{\bullet}(\mathcal{L})$ of $\mathcal{L}$. Given an $(a, b)$-form $\varphi \in \mathcal{A}^{a, b}(\mathcal{L})$, its cohomological degree in $\mathcal{C}_{\mathcal{H}}(\mathcal{L})$ is given by

$$
\operatorname{deg}(\varphi):= \begin{cases}a+b & \text { if }(s, t)=(0,0) \\ a+b+1 & \text { otherwise }\end{cases}
$$

The real Hodge complex $\mathcal{C}_{\mathcal{H} ; \mathbb{R}}^{\bullet}(\mathcal{L})$. Let $\mathcal{L}$ be a variation of real Hodge structures. Let $\mathcal{A}_{\mathbb{R}}^{*}(\mathcal{L})$ be its real de Rham complex. Its complexification is a cohomological Dolbeaut bicomplex. Applying the real Hodge complex functor $\mathcal{C}_{\mathcal{H} ; \mathbb{R}}^{\bullet}(-)$ to $\mathcal{A}_{\mathbb{R}}^{*}(\mathcal{L})$ we get the real Hodge complex of the variation of real Hodge structures $\mathcal{L}$ :

$$
\mathcal{C}_{\mathcal{H} ; \mathbb{R}}^{\bullet}(\mathcal{L}):=\mathcal{C}_{\mathcal{H} ; \mathbb{R}}^{\bullet}\left(\mathcal{A}_{\mathbb{R}}^{*}(\mathcal{L})\right)
$$

Calculating Hodge cohomology. A pure weight $w$ Hodge-Deligne complex 5 is a complex $\left(K^{\bullet}, d_{K}\right)$ with finite dimensional cohomology and the following structures:

i) Filtrations $F^{\bullet}$ and $\bar{F}^{\bullet}$ on $K^{\bullet}$ such that the differential $d_{K}$ is strictly compatible with them.

ii) The filtrations $F^{\bullet}$ and $\bar{F}^{\bullet}$ induce a pure weight $w+n$ Hodge structure on $H^{n}\left(K^{\bullet}\right)$.

Example. Our weight $w$ cohomological Dolbeaut complex $\mathcal{A}^{*, *}$ is an example of a weight $w$ Hodge-Deligne complex for the following standard filtrations:

$$
F^{p} \mathcal{A}^{*, *}=\oplus_{s \geq p} \mathcal{A}^{s, t}, \quad \bar{F}^{q} \mathcal{A}^{*, *}=\oplus_{t \geq q} \mathcal{A}^{s, t} .
$$

The $\partial \bar{\partial}$-lemma implies that the differential is strictly compatible with the filtrations $F^{\bullet}$ and $\bar{F}^{\bullet}$.

Proposition 4.4. Let $\mathcal{A}^{*, *}$ be a cohomological Dolbeaut complex. Then the Hodge complex $\mathcal{C}_{\mathcal{H}}^{\bullet}\left(\mathcal{A}^{*, *}\right)$ is quasiisomorphic to $\mathrm{RHom}_{\mathrm{MHS}}\left(\mathbb{C}(0), \mathcal{A}^{*, *}\right)$.

Proof. Given a Hodge-Deligne complex $K^{\bullet}$, we have

$$
\operatorname{RHom}_{\mathrm{MHS}}\left(\mathbb{C}(0), K^{\bullet}\right)=\operatorname{Cone}\left(\left(\bar{F}^{0} \cap W_{0}\right)\left(K^{\bullet}\right) \oplus\left(F^{0} \cap W_{0}\right)\left(K^{\bullet}\right) \longrightarrow W_{0}\left(K^{\bullet}\right)\right) .
$$

So we can apply formula (96) to calculate $\operatorname{RHom}_{\mathrm{MHS}}\left(\mathbb{C}(0), \mathcal{A}^{*, *}\right)$.

Lemma 4.5. There is a quasiisomorphism

$$
\operatorname{Cone}\left(\left(\bar{F}^{0} \cap W_{0}\right)\left(\mathcal{A}^{*, *}\right) \oplus\left(F^{0} \cap W_{0}\right)\left(\mathcal{A}^{*, *}\right) \longrightarrow W_{0}\left(\mathcal{A}^{*, *}\right)\right) \longrightarrow \mathcal{C}_{\mathcal{H}}^{\bullet}\left(\mathcal{A}^{*, *}\right) .
$$

Proof. Let $f^{*}: X^{*} \rightarrow Y^{*}$ be a morphism of complexes such that $f^{i}$ is injective for $i<k$, isomorphism for $i=k$, and surjective for $i>k$. Then there is a complex

$$
Z^{*}:=\operatorname{Coker} f^{<k}[-1] \longrightarrow \operatorname{Ker} f^{>k}
$$

with a differential $D:$ Coker $f^{k-1} \rightarrow \operatorname{Ker} f^{k+1}$ defined by a diagram chase (G8, Proposition 2.1). Thanks to Lemma 2.2 in loc. cit., the complex $Z^{*}$ is canonically isomorphic to Cone $\left(X^{*} \rightarrow Y^{*}\right)$.

\footnotetext{
${ }^{5}$ What we call here Hodge-Deligne complex traditionally should be called complex Hodge-Deligne complex.
} 
Let us apply this construction in our case. Using (95) we get

$$
\begin{aligned}
& \operatorname{Ker}\left(\left(\bar{F}^{0} \cap W_{0}\right) \oplus\left(F^{0} \cap W_{0}\right) \longrightarrow W_{0}\right)=\bar{F}^{0} \cap F^{0} \cap W_{0}=\mathcal{A}^{0,0} . \\
& \operatorname{Coker}\left(\left(\bar{F}^{0} \cap W_{0}\right) \oplus\left(F^{0} \cap W_{0}\right) \longrightarrow W_{0}\right)=\oplus_{s, t \leq-1} \mathcal{A}^{s, t} .
\end{aligned}
$$

The differential is the differential $D$. The Lemma and hence Proposition 4.4 are proved.

Similarly if $K_{\mathbb{R}}^{\bullet}$ a real Hodge-Deligne complex, the standard result [B] tells that

$$
\operatorname{RHom}_{M H S / \mathbb{R}}\left(\mathbb{R}(0), K_{\mathbb{R}}^{\bullet}\right)=\operatorname{Cone}\left(W_{0} K_{\mathbb{R}}^{\bullet} \oplus F^{0} W_{0} K_{\mathbb{C}}^{\bullet} \longrightarrow W_{0} K_{\mathbb{C}}^{\bullet}\right)
$$

Therefore a similar argument shows that there is a quasiisomorphism

$$
\operatorname{Cone}\left(W_{0} \mathcal{A}_{\mathbb{R}}^{\bullet} \oplus F^{0} W_{0} \mathcal{A}_{\mathbb{C}}^{\bullet} \longrightarrow W_{0} \mathcal{A}_{\mathbb{R}}^{\bullet}\right) \longrightarrow \mathcal{C}_{\mathcal{H}_{\mathbb{R}}}^{\bullet}\left(\mathcal{A}_{\mathbb{C}}^{\bullet}\right)
$$

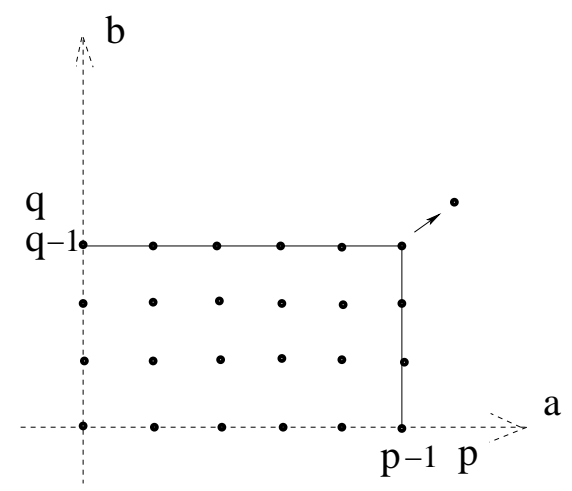

Figure 5: The de Rham bigrading $(a, b)$ on the $\mathcal{L}^{-p,-q}$-part of the Hodge complex $\mathcal{C}_{\mathcal{H}}(\mathcal{L})$.

Proposition 4.6. Let $\mathcal{L}$ be a variation of Hodge structures on a complex manifold $X$. Then the complex $\mathcal{C}_{\mathcal{H} ; \mathbb{R}}^{\bullet}(\mathcal{L})$ is quasiisomorphic to $\mathrm{RHom}_{\operatorname{Sh}_{\text {Hod }(X)}}(\mathbb{C}(0), \mathcal{L})$.

Let $\mathcal{L}_{\mathbb{R}}$ be a variation of real Hodge structures on a complex manifold $X$. Then the complex $\mathcal{C}_{\mathcal{H} ; \mathbb{R}}\left(\mathcal{L}_{\mathbb{R}}\right)$ is quasiisomorphic to $\operatorname{RHom}_{\mathrm{Sh}_{\mathbb{R}-\operatorname{Hod}(X)}}\left(\mathbb{R}(0), \mathcal{L}_{\mathbb{R}}\right)$.

Proof. We calculate $\mathrm{RHom}_{\mathrm{Sh}_{\operatorname{Hod}(X)}}(\mathbb{C}(0), \mathcal{L})$ in two steps. First, take the Dolbeaut bicomplex $\mathcal{A}^{*, *}(\mathcal{L})$ of $\mathcal{L}$. It is a Hodge-Deligne complex of weight given by the weight of $\mathcal{L}$. Then calculate $\mathrm{RHom}_{\mathrm{MHS}}\left(\mathbb{C}(0), \mathcal{A}^{*, *}(\mathcal{L})\right)$ using Proposition 4.4, The second part is similar.

\subsection{The twistor transform and the DGCom structure on Hodge complexes}

Recall the twistor plane $\mathbb{C}^{2}$ with the canonical coordinates $(z, w)$, and the twistor line $\mathbb{C}$ given by the equation $z+w=1$, with the parameter $u$ such that $z=1-u, w=u$.

Denote by $\left(\Omega_{\mathbb{C}^{2}}^{\bullet}, d_{\mathbb{C}^{2}}\right)$ the algebraic de Rham complex on $\mathbb{C}^{2}$. Set

$$
\mathcal{A}^{*, *} \otimes_{\mathbb{C}} \Omega_{\mathbb{C}^{2}}^{\bullet}
$$


There is an action of the group $\mathbb{C}^{*} \times \mathbb{C}^{*}$ on (99) given by the natural action on $\mathbb{C}^{2}$ and the action on the $\mathcal{A}^{*, *}$ provided by the Hodge bigrading: an element $(u, v) \in \mathbb{C}^{*} \times \mathbb{C}^{*}$ acts by

$$
(z, w) \longmapsto(u z, v w), \quad \varphi_{s, t} \longmapsto u^{-s} v^{-t} \varphi_{s, t}, \quad \varphi_{s, t} \in \mathcal{A}^{-s,-t} .
$$

Take the invariants of the $\mathbb{C}^{*} \times \mathbb{C}^{*}$ action:

$$
\left(\mathcal{A}^{*, *} \otimes_{\mathbb{C}} \Omega_{\mathbb{C}^{2}}^{\bullet}\right)^{\mathbb{C}^{*} \times \mathbb{C}^{*}}
$$

Definition 4.7. The homogeneous twistor transform is a linear map

$$
\begin{gathered}
\widehat{\gamma}: \mathcal{C}_{\mathcal{H}}^{\bullet}\left(\mathcal{A}^{*, *}\right) \longrightarrow\left(\mathcal{A}^{*, *} \otimes_{\mathbb{C}} \Omega_{\mathbb{C}^{2}}^{\leq 1}\right)^{\mathbb{C}^{*} \times \mathbb{C}^{*}}, \\
\widehat{\gamma}: \varphi_{0,0}+\sum_{s, t \geq 0} \varphi_{s+1, t+1} \longmapsto \\
\varphi_{0,0}+\sum_{s, t \geq 0} z^{s} w^{t}\left((z d w-w d z) \wedge(s+t+1) \varphi_{s+1, t+1}+\left(w \partial^{\prime}-z \partial^{\prime \prime}\right) \varphi_{s+1, t+1}\right) .
\end{gathered}
$$

The twistor transform $\gamma$ is its restriction to the twistor line $\mathbb{C}$ :

$$
\gamma: \mathcal{C}_{\mathcal{H}}^{\bullet}\left(\mathcal{A}^{*, *}\right) \longrightarrow \mathcal{A}^{*, *} \otimes_{\mathbb{C}} \Omega_{\mathbb{C}}^{\bullet}
$$

The homogeneous twistor transform is injective and functorial.

To formulate the reality condition for the homogeneous twistor transform, recall the complex conjugation $c$ and the involution $\sigma:(z, w) \longmapsto(\bar{w}, \bar{z})$ of the $\mathbb{C}^{2}$. So there is an involution

$$
c \otimes \sigma: \mathcal{A}^{*, *} \otimes \Omega_{\mathbb{C}^{2}}^{\bullet} \longrightarrow \mathcal{A}^{*, *} \otimes \Omega_{\mathbb{C}^{2}}^{\bullet}, \quad f \otimes \omega \longmapsto c\left(f \otimes \sigma^{*}(\omega)\right) .
$$

One easily checks the following.

\section{Lemma 4.8.}

$$
(c \otimes \sigma)^{*} \widehat{\gamma}(\varphi)=\widehat{\gamma}(\varphi) \text { if and only if } \varphi \in \mathcal{C}_{\mathcal{H} ; \mathbb{R}}^{\bullet}\left(\mathcal{A}^{*, *}\right) .
$$

Denote by the subscript + the coinvariants of the involution $c \otimes \sigma$. Then we have

$$
\widehat{\gamma}: \mathcal{C}_{\mathcal{H} ; \mathbb{R}}^{\bullet}\left(\mathcal{A}^{*, *}\right) \longrightarrow\left(\mathcal{A}^{*, *} \otimes \Omega_{\mathbb{\mathbb { C }}^{2}}^{\leq 1}\right)_{+}^{\mathbb{C}^{*} \times \mathbb{C}^{*}},
$$

The following theorem generalizes Theorem 1.9 in the Introduction.

Theorem 4.9. i) The homogeneous twistor transform $\widehat{\gamma}$ preserves the degree.

ii) The image of the twistor transform $\gamma$ is closed under the differential.

iii) Let us assume that $\mathcal{A}^{*, *}$ is a cohomological Dolbeaut DGCom. Then the image of the homogeneous twistor transform $\widehat{\gamma}$ is closed under the product.

So the image of the twistor transform $\gamma$ is a DG subalgebra of the de Rham DGCom of the twistor line:

$$
\gamma\left(\mathcal{C}_{\mathcal{H}}^{\bullet}\left(\mathcal{A}^{*, *}\right)\right) \subset \mathcal{A}^{*, *} \otimes_{\mathbb{C}} \Omega_{\mathbb{C}}^{\bullet}
$$

iv) The same true for the real twistor transform. 
First steps of the proof of Theorem 4.9. .

We have to check the following three statements:

- (i) The homogeneous twistor transform $\widehat{\gamma}$ preserves the degrees.

- (ii) Multiplicativity. Given cohomological Dolbeaut bicomplexes $\mathcal{A}^{*, *}$ and $\mathcal{B}^{*, *}$, there is a product map

$$
*: \mathcal{C}_{\mathcal{H}}^{\bullet}\left(\mathcal{A}^{*, *}\right) \otimes \mathcal{C}_{\mathcal{H}}^{\bullet}\left(\mathcal{B}^{*, *}\right) \longrightarrow \mathcal{C}_{\mathcal{H}}^{\bullet}\left(\mathcal{A}^{*, *} \otimes \mathcal{B}^{*, *}\right)
$$

making the following diagram is commutative:

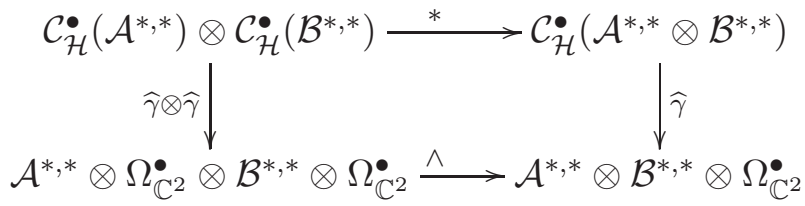

The map $\wedge$ at the bottom line uses the wedge product map $\Omega_{\mathbb{C}^{2}}^{\bullet} \wedge \Omega_{\mathbb{C}^{2}}^{\bullet} \rightarrow \Omega_{\mathbb{C}^{2}}^{\bullet}$. Thanks to the injectivity of $\widehat{\gamma}$ this implies that the map $*$ is commutative and associative.

- (iii) Differentials. There is a differential $\delta$ on the Hodge complex $\mathcal{C}_{\mathcal{H}}^{\bullet}\left(\mathcal{A}^{*, *}\right)$ such that the map $\gamma$ transforms $\delta$ into the de Rham differential $\mathbf{d}+d_{\mathbb{C}}$ on the de Rham complex $\mathcal{A}^{*, *} \otimes \Omega_{\mathbb{C}}^{\bullet}$, holomorphic along the twistor line $\mathbb{C}$, i.e. we have a commutative diagram:

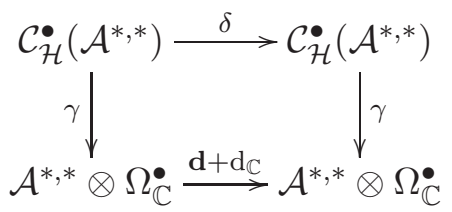

In particular, if $\mathcal{A}^{*, *}$ is an algebra in the tensor category of cohomological Dolbeaut bicomplexes, then $\mathcal{C}_{\mathcal{H}}^{\bullet}\left(\mathcal{A}^{*, *}\right)$ has a commutative differential graded algebra structure.

The property (i) is clear. Indeed, an element $\varphi_{s+1, t+1}$, whose degree in the Hodge complex is one plus its cohomological degree in the complex $\mathcal{A}^{*, *}$, is either multiplied by a 1 -form, or acted by a degree 1 differential operator. We do not change the element $\varphi_{0,0}$ whose degree in the Hodge complex coincides with its cohomological degree in $\mathcal{A}^{*, *}$.

The injectivity of the twistor transform immediately implies that the differential $\delta$ satisfies the Lebniz rule for the $*$-product.

The formulas for the product $*$ and the differential $\delta$ are not complicated. It is easy to check directly that $\delta^{2}=0$ and that the product is graded commutative. However without using the twistor transform a proof of the Leibniz rule for the $\delta$ requires a messy calculation. This is hardly surprising: hiding the role of the twistor line and the differential $d_{\mathbb{C}}$ obscures the story.

The $*$-product. $\quad$ It is handy to introduce a linear map of vector spaces preserving the grading:

$$
\mathbf{1} *: \mathcal{C}_{\mathcal{H}}^{\bullet}\left(\mathcal{A}^{*, *}\right) \longrightarrow \mathcal{A}^{*, *}, \quad \mathbf{1} * \varphi:= \begin{cases}\mathbf{d}^{\mathbb{C}} \varphi & \text { if } N_{\varphi}>0 \\ \varphi & \text { if } N_{\varphi}=0\end{cases}
$$


Its restriction to $\mathcal{C}_{\mathcal{H}, \mathbb{R}}^{\bullet}\left(\mathcal{A}^{*, *}\right)$ satisfies the reality condition:

$$
\overline{\mathbf{1} * \varphi}=\mathbf{1} * \varphi, \quad \varphi \in \mathcal{C}_{\mathcal{H}, \mathbb{R}}^{\bullet}\left(\mathcal{A}^{*, *}\right) .
$$

We use a shorthand $|\varphi|$ for the degree of $\varphi$ in the Hodge complex.

Definition 4.10. Given $\varphi_{i} \in \mathcal{C}_{\mathcal{H}}\left(\mathcal{A}_{i}^{*, *}\right)$, we define

$$
\varphi_{1} * \ldots * \varphi_{m} \in \mathcal{C}_{\mathcal{H}}\left(\mathcal{A}_{1}^{*, *} \otimes \ldots \otimes \mathcal{A}_{n}^{*, *}\right)
$$

by setting

$$
\begin{array}{cl}
\varphi_{1} * \ldots * \varphi_{m}:= & \\
\begin{cases}\varphi_{1} \otimes \ldots \otimes \varphi_{m} & \text { if } \sum N_{\varphi_{i}}=0, \\
N^{-1} \sum_{i=1}^{m}(-1)^{\left|\varphi_{1}\right|+\ldots+\left|\varphi_{i-1}\right|} \mathbf{1} * \varphi_{1} \otimes \ldots \otimes N \varphi_{i} \otimes \ldots \otimes \mathbf{1} * \varphi_{m} & \text { otherwise. }\end{cases}
\end{array}
$$

Proposition 4.11. (i) One has

$$
1 *\left(\varphi_{1} * \ldots * \varphi_{m}\right)=1 * \varphi_{1} \otimes \ldots \otimes 1 * \varphi_{m} .
$$

(ii) The product $*$ makes the diagram (4.3) commutative.

(iii) The product $*$ is a graded commutative and associative.

Proof. (i) Obvious.

(ii) The commutativity of the diagram when $\sum N_{\varphi_{i}}=0$ is obvious. Let us assume that $\sum N_{\varphi_{i}}>0$. Take the $\wedge$-product of several expressions (105). Look at its ( $\left.z d w-w d z\right)$-component. The degree $|\varphi|$ of $\varphi$ in the Hodge complex coincides with the cohomological degree of $\mathbf{1} * \varphi$ in the complex $\mathcal{A}^{*, *}$, as well as with the de Rham degree of $(z d w-w d z) \wedge N \varphi_{i}$, provided $N \varphi_{i}>0$. So moving $(z d w-w d z)$ to the left we get

$$
(z d w-w d z) \wedge \sum_{i=1}^{k}(-1)^{\left|\varphi_{1}\right|+\ldots+\left|\varphi_{i-1}\right|} \mathbf{1} * \varphi_{1} \otimes \ldots \otimes N \varphi_{i} \otimes \ldots \otimes \mathbf{1} * \varphi_{m} .
$$

This is equal to $(z d w-w d z) \wedge N\left(\varphi_{1} * \ldots * \varphi_{m}\right)$. So we recovered Definition 4.10 for the $*$-product in the case $\sum N_{\varphi_{i}}>0$. The claim that the second term in (105) is multiplicative is obvious (and equivalent to (i)).

(iii) Follows from the injectivity of the twistor transform.

\section{Explicit formulas.}

$$
\varphi_{1} * \varphi_{2}= \begin{cases}\varphi_{1} \otimes \varphi_{2} & \text { if } N_{\varphi_{1}}=N_{\varphi_{2}}=0, \\ N^{-1}\left(N \varphi_{1} \otimes \varphi_{2}+(-1)^{\left|\varphi_{1}\right|} \varphi_{1} \otimes N \varphi_{2}\right) & \text { if } N_{\varphi_{1}}=0 \text { or } N_{\varphi_{2}}=0, \\ N^{-1}\left(N \varphi_{1} \otimes \mathbf{d}^{\mathbb{C}} \varphi_{2}+(-1)^{\left|\varphi_{1}\right|} \mathbf{d}^{\mathbb{C}} \varphi_{1} \otimes N \varphi_{2}\right) & \text { but } N_{\varphi_{1}}+N_{\varphi_{2}}>0, \\ \text { if } N_{\varphi_{1}}>0, N_{\varphi_{2}}>0 .\end{cases}
$$

The twistor transforms can be written as

$$
\widehat{\gamma}: \varphi=\sum_{s, t} \varphi_{s, t} \longmapsto \sum_{s, t \geq 0} z^{s} w^{t}\left((z d w-w d z) \wedge(s+t+1) \varphi_{s+1, t+1}+(\mathbf{1} * \varphi)_{s, t}\right) .
$$


The differential $\delta$. Recall the differential $D$ from on $\mathcal{C}_{\mathcal{H}}\left(\mathcal{A}^{*, *}\right)$ defined in (92) .

Let $\mu$ be a linear map on $\mathcal{C}_{\mathcal{H}}^{\bullet}\left(\mathcal{A}^{*, *}\right)$ acting on the homogeneous components as

$$
\mu\left(\varphi_{0,0}\right):=\varphi_{0,0} ; \quad \mu\left(\varphi_{s+1, t+1}\right):=\left(\begin{array}{c}
s+t \\
s
\end{array}\right)^{-1} \varphi_{s+1, t+1} \text { if } s, t \geq 0 .
$$

Definition 4.12. $\delta:=-\mu^{-1} \circ D \circ \mu$.

We are going to give three different proofs of the part ii) of Theorem 4.9,

1) The first is a direct check, which is the most convincing. The reader can skip this proof.

2) The second contains an important characterisation of the twistor transform. Yet the corresponding computation is a version of the one in 1).

3) The third is the most conceptual. It elucidates the origin of the map $\mu$ in Definition 4.12 .

1) A direct proof of the part ii) of Theorem 4.9. We start with an explicit formula for the differential $\delta$ on $\mathcal{C}_{\mathcal{H}}\left(\mathcal{A}^{*, *}\right)$ from Definition 4.12 .

Lemma 4.13. One has

$$
\delta\left(\varphi_{0,0}\right)=0, \quad \delta\left(\varphi_{s+1, t+1}\right)= \begin{cases}\partial^{\prime \prime} \partial^{\prime} \varphi_{1,1} & \text { if }(s, t)=(0,0) \\ \frac{-1}{s+t}\left(s \partial^{\prime}+t \partial^{\prime \prime}\right) \varphi_{s+1, t+1} . & \text { if } s+t \geq 1\end{cases}
$$

Proof. The cases when $(s, t)$ is $(0,0)$ or $(1,1)$ are obvious. Otherwise we should have

$$
\delta\left(\varphi_{s+1, t+1}\right) \stackrel{?}{=} \frac{-1}{s+t}\left(s \partial^{\prime}+t \partial^{\prime \prime}\right) \varphi_{s+1, t+1} .
$$

Let us prove the claim for $\partial^{\prime}$-component. Using (108) as a definition, we have

$$
-\mu \delta^{\prime}\left(\varphi_{s+1, t+1}\right)=\left(\begin{array}{c}
s+t-1 \\
s-1
\end{array}\right)^{-1} \frac{s}{s+t} \partial^{\prime} \varphi_{s+1, t+1}=\left(\begin{array}{c}
s+t \\
s
\end{array}\right)^{-1} \partial^{\prime} \varphi_{s+1, t+1}=\partial^{\prime} \mu\left(\varphi_{s+1, t+1}\right) .
$$

The case of $\partial^{\prime \prime}$-component is similar.

Let us now prove that the twistor transform commutes with the differentials by a direct computation. We use the notation $\operatorname{Res}_{z+w=1}$ for the restriction to the line $z+w=1$. One has

$$
\begin{aligned}
\left(\mathbf{d}+d_{\mathbb{C}}\right) \gamma(\varphi)=\operatorname{Res}_{z+w} & \left(-\sum_{s, t \geq 0} z^{s} w^{t}(z d w-w d z) \wedge(s+t+1) \mathbf{d} \varphi_{s+1, t+1}\right. \\
+ & \sum_{s, t \geq 0} \mathrm{~d}_{\mathbb{C}^{2}}\left[z^{s} w^{t}\left(w \partial^{\prime}-z \partial^{\prime \prime}\right)\right] \varphi_{s+1, t+1} \\
& \left.+\sum_{s, t \geq 0} z^{s} w^{t} \mathbf{d}\left(w \partial^{\prime}-z \partial^{\prime \prime}\right) \varphi_{s+1, t+1}\right)
\end{aligned}
$$

i) Let us look first at (109) $+(110)$, which is the $\Omega_{\mathbb{C}}^{1}$-part of $\left(\mathbf{d}+d_{\mathbb{C}}\right) \gamma(\varphi)$. We examine the $\partial^{\prime}$-component - the other one is similar. Since in the Hodge complex $\partial^{\prime} \varphi_{1, t+1}=0$, we can assume $s>0$, and use the summation over $s \geq 1, t \geq 0$ :

$$
\left.\operatorname{Res}_{z+w=1} \sum_{s \geq 1, t \geq 0} z^{s-1} w^{t}(-z(s+t+1)(z d w-w d z)+s w d z+(t+1) z d w)\right) \partial^{\prime} \varphi_{s+1, t+1} .
$$


Now we substitute $z=1-u, w=u$. Then $z d w-w d z=d u$, and we get

$$
\begin{gathered}
\left.\operatorname{Res}_{z=1-u, w=u}(-z(s+t+1)(z d w-w d z)+s w d z+(t+1) z d w)\right)= \\
(-(1-u)(s+t+1)-s u+(t+1)(1-u)) d u=-s d u .
\end{gathered}
$$

Therefore we get

$$
(\underline{112})=-\sum_{s \geq 1, t \geq 0}(1-u)^{s-1} u^{t} d u \wedge s \partial^{\prime} \varphi_{s+1, t+1} .
$$

On the other hand, we have

$$
\gamma(\delta \varphi)=\operatorname{Res}_{z+w=1} \sum_{s, t \geq 0} z^{s} w^{t}\left((s+t+1)(z d w-w d z) \wedge(\delta \varphi)_{s+1, t+1}+\left(w \partial^{\prime}-z \partial^{\prime \prime}\right)(\delta \varphi)_{s+1, t+1}\right) .
$$

Using (107) we see that the $\Omega_{\mathbb{C}}^{1}$-part of its $\partial^{\prime}$-component, reduced to the $z+w=1$ line, is

$$
\begin{gathered}
-\sum_{s, t \geq 0}(1-u)^{s} u^{t}\left((s+t+1) d u \wedge \frac{(s+1)}{s+t+1} \partial^{\prime} \varphi_{s+2, t+1}\right)= \\
-\sum_{s, t \geq 0}(1-u)^{s} u^{t}\left(d u \wedge(s+1) \partial^{\prime} \varphi_{s+2, t+1}\right) .
\end{gathered}
$$

We conclude that (113) coincides with (115).

ii) Let us compare the $d u$-free part of $\left(\mathbf{d}+d_{\mathbb{C}}\right) \gamma(\varphi)$, given by (111), with the $d u$-free part of $\gamma(\delta \varphi)$. If $N_{\varphi}=0$, both are zero since $\mathbf{d} \varphi_{0,0}=0$. Otherwise this follows from a key formula

$$
\left(\mathbf{d}+d_{\mathbb{C}}\right)\left(u \partial^{\prime}-(1-u) \partial^{\prime \prime}\right) \varphi=d u \mathbf{d} \varphi+\partial^{\prime \prime} \partial^{\prime} \varphi
$$

Indeed, if $N_{\varphi}>0$, the $\left(\mathbf{d}+d_{\mathbb{C}}\right) \gamma(\varphi)$ contributes $\mathbf{d}\left(u \partial^{\prime}-(1-u) \partial^{\prime \prime}\right) \varphi=\partial^{\prime \prime} \partial^{\prime} \varphi$.

If $N_{\varphi}=1$, then $\gamma\left(\delta \varphi_{1,1}\right)=\delta \varphi_{1,1}=\partial^{\prime \prime} \partial^{\prime} \varphi_{1,1}$.

If $N_{\varphi}>1$, the $\gamma(\delta \varphi)$ contribution, calculated on a homogeneous component $\varphi=\varphi_{s, t}$ is:

$$
\left(w \partial^{\prime}-z \partial^{\prime \prime}\right) \delta \varphi=-\frac{\left(w \partial^{\prime}-z \partial^{\prime \prime}\right)}{s+t-2}\left(\frac{(s-1)}{z} \partial^{\prime}+\frac{(t-1)}{w} \partial^{\prime \prime}\right) \varphi=\partial^{\prime \prime} \partial^{\prime} \varphi .
$$

Theorem 4.9 is proved.

2) A proof of Theorem 4.9ii) via a characterisation of the twistor transform. The subspace of $\mathbb{C}^{*} \times \mathbb{C}^{*}$-invariants

$$
\left(\mathcal{A}^{*, *} \otimes \Omega_{\mathbb{C}^{2}}^{\leq 1}\right)^{\mathbb{C}^{*} \times \mathbb{C}^{*}}
$$

is not preserved by the differential $\mathbf{d}+d_{\mathbb{C}^{2}}$ : the differential $\mathbf{d}$ breaks the $\mathbb{C}^{*} \times \mathbb{C}^{*}$-invariance.

Let us consider a subspace of (118), denoted by

$$
\left(\mathcal{A}^{*, *} \otimes \Omega_{\mathbb{C}^{2}}^{\leq 1}\right)_{0}^{\mathbb{C}^{*} \times \mathbb{C}^{*}}
$$


consisting of the elements of the form

$$
\Phi_{g, \beta}(z, w):=\sum_{s, t \geq 0} z^{s} w^{t}\left((s+t+1)(z d w-w d z) g_{s+1, t+1}+\beta_{s, t}\right) .
$$

The image of the twistor transform is precisely the space of the elements $\Phi_{g, \beta}(z, w)$ such that

$$
\beta_{s, t}=\left(\mathbf{d}^{\mathbb{C}} g\right)_{s, t}, \quad(s, t) \neq(0,0) .
$$

Consider the restriction of the space (119) to the twistor line $z+w=1$ :

$$
\operatorname{Res}_{z+w=1}\left(\mathcal{A}^{*, *} \otimes \Omega_{\mathbb{C}^{2}}^{\leq 1}\right)_{0}^{\mathbb{C}^{*} \times \mathbb{C}^{*}}
$$

Denote by $\Phi_{g, \beta}(u)$ be the restriction to the twistor line of an element $\Phi_{g, \beta}(z, w)$ in (120).

Proposition 4.14. The $\left(\mathbf{d}+d_{\mathbb{C}}\right) \Phi_{g, \beta}(u)$ lies in the subspace (122) if and only if (121) holds.

The image of the twistor transform is the maximal subspace of (122) preserved by the $\mathbf{d}+d_{\mathbb{C}}$.

Proof. The second claim is a reformulation of the first. So let us prove the first one.

The $d u$-part of the differential $\left(\mathbf{d}+d_{\mathbb{C}}\right) \Phi_{g, \beta}(u)$ is given by

$$
\sum_{s, t \geq 0}(1-u)^{s} u^{t} d u \wedge\left(-(s+1) \beta_{s+1, t}+(t+1) \beta_{s, t+1}-(s+t+1) \mathbf{d} g_{s+1, t+1}\right) .
$$

We set $g_{s, t}:=0$ if $s<1$ or $t<1$, and write (123) as a sum of $(s, t)$-components:

$$
\sum_{s, t \geq 0}(1-u)^{s-1} u^{t-1} d u \wedge\left((-s u+t(1-u)) \beta_{s, t}-(s+t)\left[(1-u) \partial^{\prime} g_{s+1, t}+u \partial^{\prime \prime} g_{s, t+1}\right]\right)
$$

We stress that if $s=0$ then the $\wedge$-factor in brackets is divisible by $(1-u)$, thanks to $g_{0, t+1}=0$. Similarly for $t=0$. So we have a polynomial in $u$ for every $(s, t)$-component.

We rewrite the last formula as

$$
\underline{(123)}=\sum_{s, t}(1-u)^{s-1} u^{t-1} d u \wedge\left(u P_{s, t}+Q_{s, t}\right)
$$

where we set

$$
\begin{aligned}
& P_{s, t}:=(s+t)\left(-\beta_{s, t}+\partial^{\prime} g_{s+1, t}-\partial^{\prime \prime} g_{s, t+1}\right), \\
& Q_{s, t}:=t \beta_{s, t}-(s+t) \partial^{\prime} g_{s+1, t} .
\end{aligned}
$$

Let us compare the Hodge bidegree $(s, t)$ parts of the 1 -forms in (120) and (124). In (120), it is $g_{s, t}(1-u)^{s-1} u^{t-1} d u$ - a polynomial of degree $s+t-2$. In (124), it is $u(1-u)^{s-1} u^{t-1} d u P_{s, t}$ - a polynomial of degree $s+t-1$. Therefore $P_{s, t}=0$. This just means that, unless $s=t=0$,

$$
\beta_{s, t}=\partial^{\prime} g_{s+1, t}-\partial^{\prime \prime} g_{s, t+1}
$$

Lemma 4.15. The twistor transform intertwines the differential $\delta$ with the differential $\mathbf{d}+d_{\mathbb{C}}$. 
Proof. Substituting (126) to the formula for $Q_{s, t}$ in (125), we get

$$
Q_{s, t}:=-s \partial^{\prime} g_{s+1, t}-t \partial^{\prime \prime} g_{s, t+1} \text {. }
$$

Since $P_{s, t}=0$, comparing with (120), we see that this implies that

$$
(s+t) \delta\left(g_{s+1, t+1}\right)=-s \partial^{\prime} g_{s+1, t+1}-t \partial^{\prime \prime} g_{s+1, t+1} \text {. }
$$

But this is just the formula for the differential $\delta$ in the Hodge complex $\mathcal{C}_{\mathcal{H}}\left(\mathcal{A}^{*, *}\right)$.

Therefore the $d u$-part of the $\left(\mathbf{d}+d_{\mathbb{C}}\right) \Phi_{g, \beta}$ is given by the $d u$-part of $\Phi_{\delta g, \beta^{\prime}}$ for some $\beta^{\prime}$.

Let us check that if $\beta^{\prime}$ relates to $\delta g$ as in the twistor transform, then the $d u$-free parts of $\left(\mathbf{d}+d_{\mathbb{C}}\right) \Phi_{g, \beta}$ and $\Phi_{\delta g, \beta^{\prime}}$ coincide. To prove this, notice the following:

$$
\begin{aligned}
& \mathbf{d}\left(w \partial^{\prime}-z \partial^{\prime \prime}\right) g_{s+1, t+1}=(w+z) \partial^{\prime \prime} \partial^{\prime} g_{s+1, t+1}, \\
& \left(w \partial^{\prime}-z \partial^{\prime \prime}\right) \delta g_{s+1, t+1} \stackrel{117}{=} \partial^{\prime \prime} \partial^{\prime} g_{s+1, t+1}, \quad(s, t) \neq(0,0) .
\end{aligned}
$$

Since $\delta g_{1,1}=\partial^{\prime \prime} \partial^{\prime} g_{1,1}$, we get also the $(s, t)=(0,0)$ case.

Remark. There is a natural isomorphism of the vector spaces:

$$
H_{d_{\mathbb{C}^{2}}}^{*}\left(\mathcal{A}^{*, *} \otimes_{\mathbb{C}}\left(\Omega_{\mathbb{C}^{2}}^{0} \stackrel{d_{\mathbb{C}^{2}}}{\longrightarrow} \Omega_{\mathbb{C}^{2}}^{1}\right)\right)^{\mathbb{C}^{*} \times \mathbb{C}^{*}} \stackrel{\text { qis }}{\longrightarrow} \mathcal{C}_{\mathcal{H}}^{\bullet}\left(\mathcal{A}^{*, *}\right)
$$

Indeed, the cohomology of the complex $\Omega_{\mathbb{C}^{2}}^{0} \stackrel{d_{\mathbb{C}^{2}}}{\longrightarrow} \Omega_{\mathbb{C}^{2}}^{1}$ are represented by $1 \in \Omega_{\mathbb{C}^{2}}^{0}$ and $z^{s} w^{t}(z d w-$ $w d z) \in \Omega_{\mathbb{C}^{2}}^{1}$. So multiplying by $\mathcal{A}^{*, *}$ and taking the $\left(\mathbb{C}^{*} \times \mathbb{C}^{*}\right)$-invariants we get the space $\mathcal{C}_{\mathcal{H}}\left(\mathcal{A}^{*, *}\right)$ as defined in (91). Precisely,

$$
1 \otimes \varphi_{0,0} \longmapsto \varphi_{0,0}, \quad z^{s} w^{t}(z d w-w d z) \otimes \varphi_{s+1, t+1} \longmapsto \varphi_{s+1, t+1} .
$$

3) A proof of Theorem 4.9ii) illuminating the map $\mu$, see (106). Consider the operator of integration along the real segment $\Delta$ connecting points $(1,0)$ and $(0,1)$ in the twistor plane:

$$
\mathrm{I}:=\mathcal{A}^{*, *} \otimes \Omega_{\mathbb{C}} \longrightarrow \mathcal{A}^{*, *} .
$$

The image of the composition I $\circ \gamma$ lies in the subspace $\mathcal{C}_{\mathcal{H}}\left(\mathcal{A}^{*, *}\right) \subset \mathcal{A}^{*, *}$. So

$$
\mathrm{I} \circ \gamma: \mathcal{C}_{\mathcal{H}}^{\bullet}\left(\mathcal{A}^{*, *}\right) \longrightarrow \mathcal{C}_{\mathcal{H}}^{\bullet}\left(\mathcal{A}^{*, *}\right) \text {. }
$$

Lemma 4.16. i) One has I $\circ \gamma=\mu$ on the $N_{\varphi}>0$ subspace. Precisely,

$$
\begin{aligned}
& \text { I॰ } \gamma: \varphi_{0,0} \rightarrow 0, \\
& \text { I॰ } \gamma: \varphi_{s+1, t+1} \rightarrow\left(\begin{array}{c}
s+t \\
s
\end{array}\right)^{-1} \varphi_{s+1, t+1} \quad \text { if } s, t \geq 0 .
\end{aligned}
$$

ii) The restriction of the operator I to the image of $\gamma$ intertwines differentials:

$$
\mathrm{I} \circ\left(\mathbf{d}+\mathrm{d}_{\mathbb{C}}\right) \circ \gamma=\delta .
$$

Equivalently, there is a commutative diagram

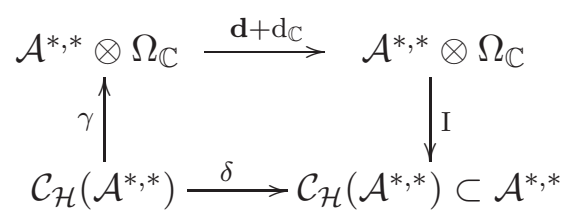


Proof. i) This follows from (70), which tells that

$$
\int_{0}^{1}(1-u)^{s} u^{t} d u=\frac{1}{s+t+1}\left(\begin{array}{c}
s+t \\
s
\end{array}\right)^{-1} .
$$

ii) Given a fibration whose fibers are compact manifolds without boundary, the integration along the fibres commutes with the differential. However in our case the fibers do have boundary.

We claim that for any $s, t \geq 0$, we have

$$
\text { I ○ } \operatorname{Res}_{z+w=1} \mathrm{~d}_{\mathbb{C}^{2}}\left(z^{s} w^{t}\left(w \partial^{\prime}-z \partial^{\prime \prime}\right) \varphi_{s+1, t+1}\right)=0 .
$$

Here $\varphi_{s+1, t+1}$ is a homogeneous element of the Hodge complex $\mathcal{C}_{\mathcal{H}}\left(\mathcal{A}^{*, *}\right)$, and $\partial^{\prime}, \partial^{\prime \prime}$ are differentials in the Hodge complex. The boundary contributions are zero for the following reasons.

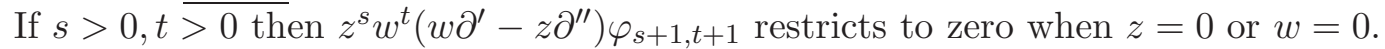

If $s=0, t>0$ then $w^{t}\left(w \partial^{\prime}-z \partial^{\prime \prime}\right) \varphi_{1, t+1}$ restricts to zero when $w=0$. It also restricts to zero when $z=0$ since $\partial^{\prime} \varphi_{1, t+1}=0$ in the Hodge complex. The case $s>0, t=0$ is similar.

Finally, in the Hodge complex $\left(w \partial^{\prime}-z \partial^{\prime \prime}\right) \varphi_{1,1}=0$.

Lemma 4.16 implies that the map I commutes with the differentials on the $\Omega_{\mathbb{C}}^{1}$-component. We use Proposition 4.14 to complete the proof.

Conclusion. We have defined a tensor functor

$$
\mathcal{C}_{\mathcal{H}}:\{\text { cohomological Dolbeaut bicomplexes }\} \longrightarrow\{\text { Complexes }\} .
$$

In particular, it restricts to a functor

$$
\mathcal{C}_{\mathcal{H}}:\{\text { Dolbeaut DGCom's }\} \longrightarrow\{\text { DGCom's }\} .
$$

\subsection{Concluding remarks}

A) The differential $\tau$. The constant function 1 on $X$ provides a canonical element

$$
\mathbf{1}_{\mathbb{R}(1)}:=1 \otimes(2 \pi i) \in \mathcal{C}_{\mathcal{H}, \mathbb{R}}^{1}(\mathbb{R}(1)) .
$$

Given a variation of $\mathbb{R}$-Hodge structures $\mathcal{L}$, there is a map provided by the $*$-product with $\mathbf{1}_{\mathbb{R}(1)}$ :

$$
\tau: \mathcal{C}_{\mathcal{H}, \mathbb{R}}^{\bullet}(\mathcal{L}) \longrightarrow \mathcal{C}_{\mathcal{H}, \mathbb{R}}^{\bullet+1}(\mathcal{L}(1)), \quad \tau \varphi:= \begin{cases}\mathbf{d}^{\mathbb{C}} \varphi \otimes 2 \pi i & \text { if } \varphi \text { homogeneous, } N_{\varphi}>0 \\ \varphi \otimes 2 \pi i & \text { if } \varphi \text { homogeneous, } N_{\varphi}=0 .\end{cases}
$$

The linear map $\mathbf{1} *$ is the $*$-product with $\mathbf{1}_{\mathbb{R}(1)}$ followed by multiplication by $(2 \pi i)^{-1}$ and the tautological linear embedding $\mathcal{C}_{\mathcal{H}, \mathbb{R}}^{\bullet+1}(\mathcal{L}) \hookrightarrow \mathcal{A}^{\bullet}(\mathcal{L})$.

Lemma 4.17. One has $\tau^{2}=0$. The map $\tau$ commutes with the differential $\delta$. So the Hodge cohomology groups form a complex:

$$
\ldots \stackrel{\tau}{\longrightarrow} \mathrm{H}_{\mathcal{H}}^{\bullet}(\mathcal{L}) \stackrel{\tau}{\longrightarrow} \mathrm{H}_{\mathcal{H}}^{\bullet+1}(\mathcal{L}(1)) \stackrel{\tau}{\longrightarrow} \mathrm{H}_{\mathcal{H}}^{\bullet+2}(\mathcal{L}(2)) \stackrel{\tau}{\longrightarrow} \ldots
$$

Proof. Clearly $\mathbf{1}_{\mathbb{R}(1)} * \mathbf{1}_{\mathbb{R}(1)}=\mathbf{d}^{\mathbb{C}} 1 \otimes(2 \pi i)^{2}=0$. This plus associativity of the $*$-product implies $\tau^{2}=0$. Since $\delta \mathbf{1}_{\mathbb{R}(1)}=0$, the second statement follows from the Leibniz rule.

A similar, yet different, complex of Weil-étale cohomologies is used by S. Lichtenbaum [Li]. 
B) Algebra structures on $\mathcal{A}_{X}^{*}[-1]$. Here is a family of graded commutative algebra structures on the shifted $C^{\infty}$ de Rham complex $\mathcal{A}_{X}^{*}[-1]$ of a complex manifold $X$. Let $N$ be a linear operator on $\mathcal{A}_{X}^{*}$ acting by a scalar on the subspace of forms of given degree: for a homogeneous form $\alpha$ we have $N(\alpha)=N_{\alpha} \cdot \alpha$. Let us assume that for any homogeneous forms $\alpha_{1}, \alpha_{2}$ we have

$$
N_{\alpha_{1} \circ \alpha_{2}}=N_{\alpha_{1}}+N_{\alpha_{2}}
$$

Let $|\alpha|$ be the degree of $\alpha$ in $\mathcal{A}_{X}^{*}[-1]$. We define a product $\circ$ on $\mathcal{A}_{X}^{*}[-1]$ by

$$
N\left(\alpha_{1} \circ \ldots \circ \alpha_{m}\right):=\sum_{i=1}^{k}(-1)^{\left|\alpha_{i}\right|\left(\left|\alpha_{1}\right|+\ldots+\left|\alpha_{i-1}\right|\right)} N\left(\alpha_{i}\right) \wedge d^{\mathbb{C}} \alpha_{1} \wedge \ldots \wedge \widehat{N\left(\alpha_{i}\right)} \wedge \ldots \wedge d^{\mathbb{C}} \alpha_{m} .
$$

Lemma 4.18. (i) One has $d^{\mathbb{C}}\left(\alpha_{1} \circ \ldots \circ \alpha_{m}\right)=d^{\mathbb{C}} \alpha_{1} \wedge \ldots \wedge d^{\mathbb{C}} \alpha_{m}$.

(ii) The product o provides $\mathcal{A}_{X}^{*}[-1]$ with a structure of a graded commutative associative algebra.

Proof. (i) Obvious thanks to (136).

(ii) The product is supercommutative by the very definition. One has, using (i),

$N\left(\left(\alpha_{1} \circ \alpha_{2}\right) \circ \alpha_{3}\right)=N\left(\alpha_{1} \circ \alpha_{2}\right) \wedge d^{\mathbb{C}} \alpha_{3}+(-1)^{\left|\alpha_{3}\right|\left(\left|\alpha_{1}\right|+\left|\alpha_{2}\right|\right)} N \alpha_{3} \wedge d^{\mathbb{C}}\left(\alpha_{1} \circ \alpha_{2}\right)=N\left(\alpha_{1} \circ \alpha_{2} \circ \alpha_{3}\right)$.

Similarly $N\left(\alpha_{1} \circ \alpha_{2} \circ \alpha_{3}\right)=N\left(\alpha_{1} \circ\left(\alpha_{2} \circ \alpha_{3}\right)\right)$.

\section{Twistor connections and variations of mixed Hodge structures}

Below we assume that $X$ is a compact complex manifold.

All results of Section 5 admit a rather straightforward generalization when $X$ is a smooth complex variety, not necessarily projective. We use a regular compactification of $X$ with a normal crossing divisor at infinity and the standard technique to extend our results.

Definition 5.1. A variation of mixed Hodge structure 6 over a complex manifold $X$ is a complex local system $(\mathcal{L}, \nabla)$ on $X$ equipped with three filtrations: $W_{\bullet}, F^{\bullet}, \bar{F}^{\bullet}$ such that

$$
\begin{gathered}
\operatorname{gr}_{F}^{p} \operatorname{gr}_{F}^{q} \operatorname{gr}_{n}^{W}=0 \text { unless } p+q=n . \\
\nabla\left(F^{p}\right) \subset F^{p-1} \otimes \Omega_{X}^{1} \oplus F^{p} \otimes \bar{\Omega}_{X}^{1} . \\
\nabla\left(\bar{F}^{q}\right) \subset \bar{F}^{q-1} \otimes \bar{\Omega}_{X}^{1} \oplus F^{q} \otimes \Omega_{X}^{1} .
\end{gathered}
$$

The condition (138) is the Griffiths condition for $F^{\bullet}$. It is equivalent to the Griffiths transversality for $F^{\bullet}$ plus holomorphicity of $F^{\bullet}$. Similarly (139) is the Griffiths condition for $\bar{F}^{\bullet}$.

The Galois group $\operatorname{Gal}(\mathbb{C} / \mathbb{R})$ acts on the category MHodx of variations of mixed Hodge structures. Its generator acts by a functor

$$
\left(X, \mathcal{L}, \nabla ; W_{\bullet}, F^{\bullet}, \bar{F}^{\bullet}\right) \longrightarrow\left(\bar{X}, \mathcal{L}, \nabla ; W_{\bullet}, \bar{F}^{\bullet}, F^{\bullet}\right) .
$$

\footnotetext{
${ }^{6}$ What we call a variation of mixed Hodge structures is usually called a variation of complex mixed Hodge structures.
} 
The category $\operatorname{MHod}_{\mathrm{X}}^{\mathbb{R}}$ is the category of $\operatorname{Gal}(\mathbb{C} / \mathbb{R})$-equivariant objects in the category MHodx.

The category $\operatorname{Hod}_{X}$ of variations of Hodge structures on $X$ is a semi-simple abelian tensor category. The category $\operatorname{MHod}_{X}$ of variations of mixed Hodge structures on $X$ is an abelian tensor category. It is a mixed category, equipped with a canonical tensor functor

$$
\operatorname{gr}_{\bullet}^{W}: \operatorname{MHod}_{X} \longrightarrow \operatorname{Hod}_{X} ; \quad \mathcal{L} \longmapsto \operatorname{gr}_{\bullet}^{W} \mathcal{L} .
$$

There is a canonical bigrading

$$
\operatorname{gr}_{\bullet}^{W} \mathcal{L}=\oplus_{p, q}\left(\operatorname{gr}_{\bullet}^{W} \mathcal{L}\right)^{p, q} .
$$

Our goal is to equip the object (140) with an additional Green datum, which allows to recover the original variation of mixed Hodge structures in a functorial way, compatible with the $\mathrm{Gal}(\mathbb{C} / \mathbb{R})$ action. This implies a similar description of variations of mixed $\mathbb{R}$-Hodge structures.

\subsection{Green data and twistor connections}

The DG Lie algebra $\mathcal{C}_{\mathcal{H}}^{\bullet}($ End $\mathcal{V})$. Let $\mathcal{L}$ be variation of Hodge structures on $X$ with a Lie algebra structure. Then the Hodge complex $\mathcal{C}_{\mathcal{H}}^{\bullet}(\mathcal{L})$ has a DG Lie algebra structure: the commutator is given by the composition

$$
\mathcal{C}_{\mathcal{H}}^{\bullet}(\mathcal{L}) \otimes \mathcal{C}_{\mathcal{H}}^{\bullet}(\mathcal{L}) \stackrel{\text { *-product }}{\longrightarrow} \mathcal{C}_{\mathcal{H}}^{\bullet}(\mathcal{L} \otimes \mathcal{L}) \stackrel{[*, *]}{\longrightarrow} \mathcal{C}_{\mathcal{H}}^{\bullet}(\mathcal{L})
$$

To prove the Jacobi identity, observe that a triple commutator is induced by the composition

$$
\mathcal{C}_{\mathcal{H}}^{\bullet}(\mathcal{L}) \otimes \mathcal{C}_{\mathcal{H}}^{\bullet}(\mathcal{L}) \otimes \mathcal{C}_{\mathcal{H}}^{\bullet}(\mathcal{L}) \stackrel{*}{\longrightarrow} \mathcal{C}_{\mathcal{H}}^{\bullet}(\mathcal{L} \otimes \mathcal{L} \otimes \mathcal{L}) \stackrel{[*, *], *]}{\longrightarrow} \mathcal{C}_{\mathcal{H}}^{\bullet}(\mathcal{L})
$$

The Jacoby identity follows from three facts:

i) The $*$-product is associative.

ii) The Hodge complex $\mathcal{C}_{\mathcal{H}}^{\bullet}(\mathcal{L})$ is functorial.

iii) The commutator [,] is a morphism of Hodge structures. It satisfies the Jacoby identity.

Similarly, if $\mathcal{L}$ is a variation of Hodge structures on $X$ with an algebra structure, then the Hodge complex $\mathcal{C}_{\mathcal{H}}^{\bullet}(\mathcal{L})$ has a DG algebra structure.

Let $\mathcal{V}$ be a variation of Hodge structures on $X$. Let us introduce a DG algebra of derived Hodge endomorphisms of $\mathcal{V}$. Let End $\mathcal{V}$ be the local system of endomorphisms of the local system $\mathcal{V}$. Since it is an algebra, the Hodge complex $\mathcal{C}_{\mathcal{H}}^{\bullet}(\operatorname{End} \mathcal{V})$ is a DG algebra.

Definition 5.2. A Green datum on a variation of Hodge structures $\mathcal{V}$ is a degree 1 element

$$
\mathcal{G} \in \mathcal{C}_{\mathcal{H}}^{1}(\operatorname{End} \mathcal{V})
$$

of the DG Lie algebra $\mathcal{C}_{\mathcal{H}}^{\bullet}($ End $\mathcal{V})$ which satisfies a Maurer-Cartan equation

$$
\delta \mathcal{G}+\mathcal{G} \wedge \mathcal{G}=0 .
$$


Explicit formulas. Let us elaborate Definition 5.2. The bigrading on $\mathcal{V}$ induces a bigrading on the local system End $\mathcal{V}$ : its bidegree $(-p,-q)$ component End $^{-p,-q} \mathcal{V}$ consists of linear maps $A$ such that $A \mathcal{V}^{a, b} \subset \mathcal{V}^{a-p, b-q}$.

Lemma 5.3. An element $\mathcal{G} \in \mathcal{C}_{\mathcal{H}}^{1}(\operatorname{End} \mathcal{V})$ is given by the following datum:

- A collection of linear operators

$$
G_{s, t} \in \mathrm{End}^{-s,-t} \mathcal{V}_{\infty}, \quad s, t \geq 1
$$

- A closed 1-form with values in EndV

$$
g_{0,0} \in \mathcal{A}^{1,0} \otimes \operatorname{End}^{-1,0} \mathcal{V}_{\infty} \oplus \mathcal{A}^{0,1} \otimes \operatorname{End}^{0,-1} \mathcal{V}_{\infty}, \quad \mathbf{d} g_{0,0}=0
$$

We call the operator $G=\sum_{s, t \geq 1} G_{s, t}$ the Green operator.

Proof. An element of $\mathcal{C}_{\mathcal{H}}^{1}(\operatorname{End} \mathcal{V})$ is given by a pair $\left\{G, g_{0,0}\right\}$, where $G$ is a function, $g_{0,0}$ is a 1 -form with the values End $\mathcal{V}$. Since $G$ is a function, its components have the Hodge bidegree $(-s,-t)$, where $(s, t) \geq(1,1)$. So we recover conditions (143).

Since $g_{0,0}$ is a 1 -form of degree 1 in the complex $\mathcal{C}_{\mathcal{H}}^{\bullet}(\operatorname{End} \mathcal{V})$, its Hodge bidegree is $(0,0)$. So it is closed. If a component of the 1 -form $g_{0,0}$ lies in $\mathcal{A}^{a, b} \otimes \operatorname{End}^{-p,-q} \mathcal{V}$ then $(0,0)=(a-p, b-q)$. This imples conditions (144). Clearly these conditions are not only necessary, but also sufficient.

Twistor connections. Recall the projection $\pi: X \times \mathbb{C}^{2} \rightarrow X$. An element $\mathcal{G} \in \mathcal{C}_{\mathcal{H}}^{1}($ End $\mathcal{V})$ gives rise, via the twistor transform $\gamma$, to a connection $\nabla_{\mathcal{G}}$ on $\pi^{*} \mathcal{V}_{\infty}$ :

$$
\begin{aligned}
& \nabla_{\mathcal{G}}:=\mathbf{d}+d_{\mathbb{C}^{2}}+\gamma(\mathcal{G}) \\
& =\mathbf{d}+d_{\mathbb{C}^{2}}+g_{0,0}+\sum_{s, t \geq 0} z^{s} w^{t}\left((s+t+1) G_{s+1, t+1}(z d w-w d z)+\left(w \partial^{\prime}-z \partial^{\prime \prime}\right) G_{s+1, t+1}\right) .
\end{aligned}
$$

Recall the twistor line $\mathbb{C} \subset \mathbb{C}^{2}$, given by $z+w=1$. The crucial fact about it is the following:

Proposition 5.4. An element $\mathcal{G} \in \mathcal{C}_{\mathcal{H}}^{1}($ End $\mathcal{V})$ on a variation of Hodge structures $\mathcal{V}$ satisfies the Maurer-Cartan equation (142) if and only if the connection $\nabla_{\mathcal{G}}$ on $X \times \mathbb{C}$ is flat.

Proof. Follows immediately from Theorem 1.9.

Definition 5.5. The connections $\nabla_{\mathcal{G}}$ on $X \times \mathbb{C}^{2}$ as in 145) such that the restriction $\operatorname{Res}_{z+w=1} \nabla_{\mathcal{G}}$ is flat arew called twistor connection.

The Green data and twistor connections are two ways to look at the same object.

Tensor product of two flat twistor connections is again a flat twistor connection. Therefore flat twistor connections, or, equivalently, Green data, form an abelian tensor category $\mathcal{G}_{\mathcal{H}}(X)$.

Explicitly, the objects of the category $\mathcal{G}_{\mathcal{H}}(X)$ are pairs $(\mathcal{V}, \mathcal{G})$, where $\mathcal{V}$ is a variation of Hodge structures on $X$ and $\mathcal{G}$ is a Green datum on it. The morphisms are morphisms of variations of Hodge structures $\mathcal{V}_{1} \rightarrow \mathcal{V}_{2}$ commuting with the Green data. The tensor product is given by $\left(\mathcal{V}_{1}, \mathcal{G}_{1}\right) \otimes\left(\mathcal{V}_{2}, \mathcal{G}_{2}\right)=\left(\mathcal{V}_{1} \otimes \mathcal{V}_{2}, \mathcal{G}_{1} \otimes \operatorname{Id}_{\mathcal{V}_{2}}+\operatorname{Id}_{\mathcal{V}_{1}} \otimes \mathcal{G}_{2}\right)$

Lemma 5.6. The category $\mathcal{G}_{\mathcal{H}}(X)$ is canonically equivalent to the category of comodules over the Lie coalgebra $\mathcal{L}_{\mathcal{H} ; X}$ in the category $\operatorname{Hod}_{X}$.

We prove this lemma in Section 5.6 below. We will not use it before.

Theorem 5.7. One has a canonical equivalence between the tensor category of variations of mixed Hodge structures on $X$ and the tensor category $\mathcal{G}_{\mathcal{H}}(X)$ of twistor connections on $X$. 


\subsection{Deligne's description of triples of opposite filtrations}

For the convenience of the reader we reproduce Deligne's approach to mixed (complex) Hodge structures, presenting it, following [D2, in a bit more general set-up.

Let $\mathcal{A}$ be an abelian category. Let $A$ be an object of $\mathcal{A}$ with three finite complimentary filtrations $W_{\bullet}, F^{\bullet}, \bar{F}^{\bullet}$, which means that

$$
\operatorname{Gr}_{F}^{p} \operatorname{Gr}_{F}^{q} \operatorname{Gr}_{n}^{W} A=0 \text { if } n \neq p+q .
$$

Here the $W_{\bullet}$ is an increaing filtration, and the other two are decreasing. This just means that filtrations $F^{\bullet}, \bar{F}^{\bullet}$ induce $n$-opposite filtrations on the $\operatorname{Gr}_{n}^{W} A$, providing a decomposition

$$
\mathrm{Gr}_{n}^{W} A=\bigoplus_{p+q=n} A_{W}^{p, q}
$$

such that the filtrations on $\operatorname{Gr}_{n}^{W} A$ induced by the ones $F^{\bullet}$ and $\bar{F}^{\bullet}$ are given by

$$
F^{i} \operatorname{Gr}_{n}^{W} A=\bigoplus_{p+q=n, p \geq i} A_{W}^{p, q}, \quad \bar{F}^{i} \operatorname{Gr}_{n}^{W} A=\bigoplus_{p+q=n, q \geq i} A_{W}^{p, q} .
$$

Equivalently, one has $A_{W}^{p, q}=F^{p} \mathrm{Gr}_{n}^{W} A \cap \bar{F}^{q} \mathrm{Gr}_{n}^{W} A$.

Any bigraded object $B=\bigoplus_{p+q} B^{p, q}$ has three canonical filtrations $W, F_{W}$, and $\bar{F}_{W}$ :

$$
W_{n}:=\bigoplus_{p+q \leq n} B^{p, q}, \quad F_{W}^{i}:=\bigoplus_{p \geq i} B^{p, q}, \quad \bar{F}_{W}^{j}:=\bigoplus_{q \geq j} B^{p, q}
$$

The filtrations $F, \bar{F}$ induce on the bigraded object $\operatorname{Gr}^{W} A=\bigoplus_{p, q} A_{W}^{p, q}$ the filtrations $F_{W}, \bar{F}_{W}$.

Deligne considers the following subobjects of the object $A$ :

$$
\begin{aligned}
& A_{F}^{p, q}:=\left(W_{p+q} \cap F^{p}\right) \cap\left(\left(W_{p+q} \cap \bar{F}^{q}\right)+\sum_{i \geq 0}\left(W_{p+q-i} \cap \bar{F}^{q-i+1}\right)\right), \\
& A_{F}^{p, q}:=\left(W_{p+q} \cap \bar{F}^{q}\right) \cap\left(\left(W_{p+q} \cap F^{p}\right)+\sum_{i \geq 0}\left(W_{p+q-i} \cap F^{p-i+1}\right)\right) .
\end{aligned}
$$

It is proved in [D1, 1.2.8], that if $n=p+q$ then the canonical projection $W_{n} A \rightarrow \operatorname{Gr}_{n}^{W} A$ induces an isomorphism of $A_{F}^{p q} \stackrel{\sim}{\rightarrow} A_{W}^{p q}$ Th This implies that the subobjects $\left\{A_{F}^{p q}\right\}$ provide a bigrading of $A$. Therefore the sum of isomorphisms $a_{F}: A_{F}^{p, q} \stackrel{\sim}{\rightarrow} A_{W}^{p, q}$ gives rise to a bifiltered isomorphism

$$
a_{F}:(A ; W, F) \longrightarrow\left(\mathrm{Gr}^{W} A ; W, F_{W}\right) .
$$

Similarly the subobjects $\left\{A_{\bar{F}}^{p q}\right\}$ give rise to a bifiltered isomorphism

$$
a_{\bar{F}}:(A ; W, \bar{F}) \longrightarrow\left(\mathrm{Gr}^{W} A ; W, \bar{F}_{W}\right) .
$$

Then the automophism $\mathrm{d}=a_{\bar{F}} a_{F}^{-1}$ of $\mathrm{Gr}^{W} A$ satisfies

$$
(\mathrm{d}-1)\left(A_{W}^{p q}\right) \subset \bigoplus_{r<p, s<q} A_{W}^{r s} .
$$

\footnotetext{
${ }^{7}$ Let us scetch the argument when $\mathcal{A}$ is a category of finite dimensional vector spaces. One has

$$
W_{p+q-1}=W_{p+q-1} \cap F^{p} \oplus\left(\sum_{i \geq 1}\left(W_{p+q-i} \cap \bar{F}^{q-i+1}\right) .\right.
$$
}

This implies that $\operatorname{dim} A_{F}^{p, q}=\operatorname{dim} A_{W}^{p, q}$, and the map $A_{F}^{p q} \rightarrow A_{W}^{p q}$ is injective. Therefore it is an isomorphism. 
Theorem 5.8. The functor $A \longrightarrow \mathrm{Gr}^{W}$ A provides an equivalence of the category of objects of $\mathcal{A}$ equipped with three complimentary filtrations $\left(W_{\bullet}, F^{\bullet}, \bar{F}^{\bullet}\right)$ with the category of bigraded objects of $\mathcal{A}$ equipped with an automorphism d satisfying (147).

\subsection{Proof of Theorem 5.7}

1. Constructing a tensor functor $\mathcal{F}$. Let us define a functor

$$
\mathcal{F}: \mathcal{G}_{\mathcal{H}}(X) \longrightarrow \operatorname{MHod}_{X}
$$

Recall a twistor connection $\nabla_{\mathcal{G}}$ on $\pi^{*} \mathcal{V}_{\infty}$ :

$$
\nabla_{\mathcal{G}}=\mathbf{d}+\mathrm{d}_{\mathbb{C}^{2}}+g_{0,0}+\sum_{s, t \geq 0} z^{s} w^{t}\left((s+t+1) G_{s+1, t+1}(z d w-w d z)+\left(w \partial^{\prime}-z \partial^{\prime \prime}\right) G_{s+1, t+1}\right) .
$$

Let us define a variation of mixed Hodge structures on $X$ starting from the connection $\nabla_{\mathcal{G}}$.

(i) The flat connection. Restricting the connection $\nabla_{\mathcal{G}}$ to $X \times\left\{\frac{1}{2}, \frac{1}{2}\right\}$ we get a flat connection $\nabla_{\mathcal{G}}^{\left\{\frac{1}{2}\right\}}$ on the vector bundle $\mathcal{V}_{\infty}$ on $X$.

(ii) The weight filtration $W_{\bullet}$. It is the standard weight filtration on a bigraded object:

$$
W_{n} \mathcal{V}_{\mathbb{C}}:=\oplus_{p+q \leq n} \mathcal{V}^{p, q}
$$

The connection $\nabla_{\mathcal{G}}^{\left\{\frac{1}{2}\right\}}$ preserves the weight filtration.

(iii) The Hodge filtrations $F^{\bullet}$ and $\bar{F}^{\bullet}$. Consider the standard Hodge filtration on the restriction of $\pi^{*} \mathcal{V}_{\infty}$ to $X \times\{0,1\}$ :

$$
F_{\mathrm{st}}^{p}:=\oplus_{i \geq p} \mathcal{V}^{i, *} .
$$

Consider the standard conjugate Hodge filtration on the restriction of $\pi^{*} \mathcal{V}_{\infty}$ to $X \times\{1,0\}$ :

$$
\bar{F}_{\mathrm{st}}^{q}:=\oplus_{j \geq q} \mathcal{V}^{*, j} .
$$

Since the restriction of the twistor connection to the coordinate cross $z w=0$ is trivial, the weight filtration and the standard Hodge filtrations are extended canonically to the fiber over the coordinate cross.

Let $P$ be the operator of parallel transport for the connection $\nabla_{\mathcal{G}}$ along the twistor line $z+w=1$ from $X \times\{0,1\}$ to $X \times\left\{\frac{1}{2}, \frac{1}{2}\right\}$, and $\bar{P}$ a similar one from $X \times\{1,0\}$ to $X \times\left\{\frac{1}{2}, \frac{1}{2}\right\}$.

Definition 5.9. The Hodge filtrations $F^{\bullet}$ and $\bar{F}^{\bullet}$ on the $\mathcal{V}$ are the images of the corresponding standard Hodge filtrations by the operators $P$ and $\bar{P}$ :

$$
F^{p}:=P\left(F_{\mathrm{st}}^{p}\right), \quad \bar{F}^{q}:=\bar{P}\left(\bar{F}_{\mathrm{st}}^{q}\right) .
$$

Lemma 5.10. The data $\left(\mathcal{V}, \nabla_{\mathcal{G}}^{\left\{\frac{1}{2}\right\}} ; W_{\bullet}, F^{\bullet}, \bar{F}^{\bullet}\right)$ is a variation of mixed Hodge structures on $X$.

Proof. The only thing we have to prove is that the Hodge filtrations satisfy the Griffiths condition. We employ the following general observation. 
Lemma 5.11. Let $(E, \nabla)$ be a smooth flat bundle on $X \times \mathbb{R}$, and $\mathcal{F}^{\bullet}$ a filtration on $E$ invariant under the parallel transport along the $\mathbb{R}$-lines. Then if the restriction of $\left(E, \mathcal{F}^{\bullet}, \nabla\right)$ to $X \times\{s\}$ satisfies the Griffiths condition for a single $s \in \mathbb{R}$, the same is true for any $s$.

We apply Lemma 5.11 to the bundle $E=\pi^{*} \mathcal{V}_{\infty}$ with the connection $\nabla_{\mathcal{G}}$ restricted to the line $z+w=1$ and filtrations $\mathcal{F}^{\bullet}$ and $\overline{\mathcal{F}}^{\bullet}$ on $X$ given by Hodge filtrations $F^{\bullet}$ and $\bar{F}^{\bullet}$ in (151).

The standard Hodge filtration (149) on the restriction $\nabla_{\mathcal{G}}^{\{0,1\}}$ of the connection $\nabla_{\mathcal{G}}$ to $X \times\{0,1\}$ satisfies the Griffiths condition. This just means that the Hodge bidegree $(-s,-t)$ component of $\nabla_{\mathcal{G}}^{\{0,1\}}$ is zero if $s>0$. To check this notice that in the restriction to $X \times\{0,1\}$ monomials $z^{s} w^{t}$ with $s>0$ vanish.

Similarly the Griffiths condition for $\bar{F}^{\bullet}$ is clear for the restriction to $X \times\{1,0\}$.

Since the connection $\nabla_{\mathcal{G}}$ is flat over $X \times\{z+w=1\}$, by Lemma 5.11 the filtrations $F^{\bullet}$ and $\bar{F}^{\bullet}$ in (151) at $\left\{\frac{1}{2}, \frac{1}{2}\right\}$ satisfy the Griffiths condition.

2. The functor $\mathcal{F}$ is an equivalence. The functor $\mathcal{F}$ is clearly a tensor functor. To show that the functor $\mathcal{F}$ is an equivalence of categories we have to show that it is an isomorphism on Hom's, and every variation of mixed Hodge structures is obtained this way. The former claim is clear since it is known when $X$ is a point. So it remains to prove the latter.
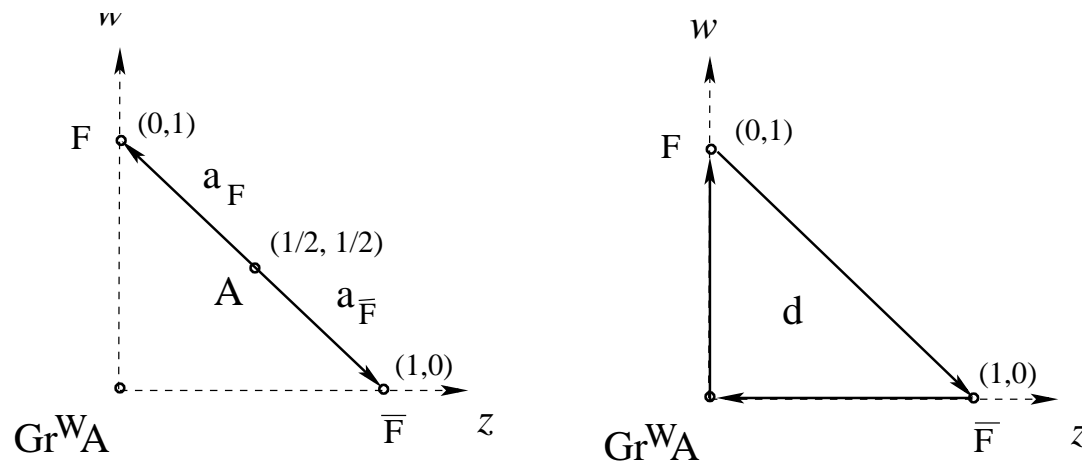

Figure 6: Deligne's operators $a_{F}, a_{\bar{F}}$, d via the homogeneous twistor connection

Fig 6 tells how Deligne's operators $a_{F}, a_{\bar{F}}$, d are expressed via the twistor connection: The object $A$ is the fiber over $\left\{\frac{1}{2}, \frac{1}{2}\right\}$.

The associate graded $\mathrm{Gr}^{W} A$ is the fiber over $(0,0)$. Since the restriction of the connection to the coordinate cross $z w=0$ is zero, the $\mathrm{Gr}^{W} A$ is identified the fiber over the coordinate cross.

The operator $a_{F}: A \rightarrow \mathrm{Gr}^{W} A$ is the parallel transport from $\left\{\frac{1}{2}, \frac{1}{2}\right\} \rightarrow\{0,1\}$.

The operator $a_{\bar{F}}: A \rightarrow \operatorname{Gr}^{W} A$ is the parallel transport from $\left\{\frac{1}{2}, \frac{1}{2}\right\} \rightarrow\{1,0\}$.

Deligne's operator $\mathrm{d}:=a_{\bar{F}} a_{F}^{-1}: \mathrm{Gr}^{W} A \rightarrow \mathrm{Gr}^{W} A$ is the clockwise monodromy around the triangle $\{0,0\} \rightarrow\{0,1\} \rightarrow\{1,0\} \rightarrow\{0,0\}$.

Identifying $\operatorname{Gr}^{W} A$ with the fiber over $z w=0$, the $\mathrm{d}$ is the parallel transport $\{0,1\} \rightarrow\{1,0\}$.

Proposition 5.12. For any variation $\mathcal{L}$ of $M H S$ there is a unique smooth family of Green operators $G_{x}$ on $\mathrm{gr}^{W} \mathcal{L}_{x}$ describing the $M H S$ 's fiberwise.

Proof. Over a point Deligne's operator d is recovered from $G$, and vice versa. Indeed, let $\mathbb{P}$ be the operator of parallel transport from $\{1,0\}$ to $\{0,1\}$. Then

$$
\mathrm{d}=\mathbb{P} .
$$


The operator $\mathbb{P}$ is given explicitly by

$$
\mathbb{P}=1+\sum_{n>0}\left(\int_{0}^{1} \varphi_{p_{1}, q_{1}} \circ \ldots \circ \varphi_{p_{n}, q_{n}}\right) G_{p_{1}+1, q_{1}+1} \ldots G_{p_{n}+1, q_{n}+1} .
$$

Here $\varphi_{p, q}:=(p+q+1)(1-u)^{p} u^{q} d u$, and $\int_{0}^{1} \varphi_{p_{1}, q_{1}} \circ \ldots \circ \varphi_{p_{n}, q_{n}}$ is a positive rational number given by the iterated integral of the 1 -forms $\varphi_{p_{1}, q_{1}}, \ldots, \varphi_{p_{n}, q_{n}}$. Therefore we have

$$
g_{p, q}:=\log (\mathrm{d})=\log (\mathbb{P})_{p, q}=\int_{0}^{1} \varphi_{p-1, q-1} G_{p, q}+\ldots
$$

where ... denotes a polynomial with rational coefficients of degrees $\geq 2$ in $G_{*, *}$, of the Hodge bidegree $(-p,-q)$. By the induction $G_{p, q}$ is recovered from $g_{*, *}$.

Thus there is a unique isomorphisms of fibrations

$$
\eta: \operatorname{gr}^{W} \mathcal{L} \stackrel{\sim}{\rightarrow} \mathcal{L}
$$

such that $\operatorname{gr}^{W} \eta: \operatorname{gr}^{W} \mathcal{L} \rightarrow \operatorname{gr}^{W} \mathcal{L}$ is the identity map, and which identifies pointwise the MHS provided by the pair $\left(\operatorname{gr}^{W} \mathcal{L}_{x}, G_{x}\right)$ with the one $\mathcal{L}_{x}$.

Let $\mathbf{d}=\partial^{\prime}+\partial^{\prime \prime}$ be the connection on $\mathrm{gr}^{W} \mathcal{L}$ induced by the connection on $\mathcal{L}$. The isomorphism $\eta^{-1}$ transforms the connection on $\mathcal{L}$ to another connection $\nabla$ on $\operatorname{gr}^{W} \mathcal{L}$. We have to show that:

The connection on $\mathcal{L}$ determines uniquely a 1-form $g_{0,0}$ as in 144.).

The Griffiths conditions for $F^{\bullet}$ and $\bar{F}$ are equivalent to the following two conditions:

1. One has

$$
\nabla=\nabla_{\mathcal{G}}^{\frac{1}{2}}
$$

2. The operators $G_{x}$ and the 1-form $g_{0,0}$, organised into a Green data $\mathcal{G}=\left(G, g_{0,0}\right)$, satisfy

$$
\delta \mathcal{G}+\mathcal{G} \wedge \mathcal{G}=0 .
$$

Write the connection $\nabla$ as follows, where the $\mathcal{B}_{s, t}$ is a 1 -form of the Hodge bidegree $(-s,-t)$ :

$$
\nabla=\mathbf{d}+\mathcal{B} ; \quad \mathcal{B}=\sum_{s, t} 2^{-(s+t)} \mathcal{B}_{s, t}
$$

Consider the third quadrant in the $(s, t)$-plane, shown on Fig. 7

$$
B=\{(s, t) \mid s, t \leq 0\}
$$

Lemma 5.13. $\mathcal{B}_{s, t}=0$ unless $(s, t) \in-B$.

Proof. Since the connection $\nabla$ preserves the weight filtration, its components of positive weights are zero. The weight zero component of $\nabla$ is the connection $\mathbf{d}$ on $\operatorname{gr}^{W} \mathcal{L}$.

The Griffiths condition for the filtrations $F^{\bullet}$ and $\bar{F}^{\bullet}$ on the bundle with connection $\mathbf{d}+\mathcal{B}$ just mean that

$$
\left(P^{-1} \mathbf{d} P+P^{-1} \mathcal{B} P\right)_{s, t}=0 \text { if } s>0 .
$$




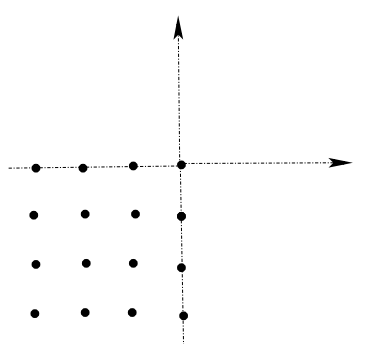

Figure 7: The Hodge bidegrees of the components of the 1-form $\mathcal{B}$ must be in the domain $B$.

$$
\left(\bar{P}^{-1} \mathbf{d} \bar{P}+\bar{P}^{-1} \mathcal{B} \bar{P}\right)_{s, t}=0 \text { if } t>0 .
$$

It is clear from (152) that:

a) Operators $P-\operatorname{Id}$ and $P^{-1}-\mathrm{Id}$ are sums of the terms with $s, t \geq 1$.

b) The components of $P^{-1} \mathbf{d} P$ are inside of the domain $-B$.

This plus similar arguments for the filtration $\bar{F}^{\bullet}$ using (156) imply the Lemma.

Let us prove (153). Set $g_{0,0}:=\mathcal{B}_{0,0}$. The Hodge bidegree $(0,0)$ case follows from Lemma 5.13 .

Consider the following cousin of the twistor connection on $X \times \mathbb{C}$ :

$$
\nabla_{\mathcal{B}, \mathcal{G}}=\mathbf{d}+d_{\mathbb{C}}+\sum_{s, t \geq 0}(1-u)^{s} u^{t}\left((s+t+1) G_{s+1, t+1} d u+\mathcal{B}_{s, t}\right) .
$$

Its curvature has two components: the horizontal one, and the vertical one, which contains $d u$.

Proposition 5.14. The du-part of the curvature of the connection $\nabla_{\mathcal{B}, \mathcal{G}}$ is zero if and only if the Griffiths condition for filtrations $F^{\bullet}$ and $\bar{F}^{\bullet}$ for the connection $\mathbf{d}+\mathcal{B}$ hold.

Proof. Since the vertical components of connections $\nabla_{\mathcal{B}, \mathcal{G}}$ and $\nabla_{\mathcal{G}}$ are the same, the operator $P$ is also the operator of parallel transform from $\{0,1\}$ to $\left\{\frac{1}{2}, \frac{1}{2}\right\}$ for the connection $\nabla_{\mathcal{B}, \mathcal{G}}$. The connection $\mathbf{d}+\mathcal{B}$ is the restriction of $\nabla_{\mathcal{B}, \mathcal{G}}$ to $X \times\left\{\frac{1}{2}, \frac{1}{2}\right\}$. The value of $P^{-1}(\mathbf{d}+\mathcal{B}) P$ on a tangent vector $v$ at $(x ; 0,1)$ is a linear endomorphism of the fiber of $\pi^{*} \mathcal{V}$ at $(x ; 0,1)$. It is the composition of the parallel transform from $\{0,1\}$ to $\left\{\frac{1}{2}, \frac{1}{2}\right\}$, followed by the infinitesimal parallel transform along $v$, and then by the parallel transform back from $\left\{\frac{1}{2}, \frac{1}{2}\right\}$ to $\{0,1\}$ for the connection $\nabla_{\mathcal{B}, \mathcal{G}}$. So it is the parallel transport for the connection $\nabla_{\mathcal{B}, \mathcal{G}}$ along the three sides of an infinitesimal rectangle $R$ with the sides $v$ and $x \times[0,1 / 2]$. Thus it differs from the value of the connection $\nabla_{\mathcal{B}, \mathcal{G}}$ on $v$ by the integral of the curvature over $R$.

The restriction of the connection $\nabla_{\mathcal{B}, \mathcal{G}}$ to $X \times\{0,1\}$ (respectively $X \times\{1,0\}$ ) satisfies the Griffiths condition for $F^{\bullet}$ (respectively $\bar{F}^{\bullet}$ ) since monomials $z^{s} w^{t}$ with $s>0$ (respectively $t>0$ ) restrict to zero. Therefore using (155) (respectively (156)) we see that the Griffiths condition for $F^{\bullet}$ (respectively $\bar{F}^{\bullet}$ ) is equivalent to the condition that the integral of the $d u$-part of the curvature $\left(\nabla_{\mathcal{B}, \mathcal{G}}\right)^{2}$ over the interval $\frac{1}{2} \leq u \leq 1$ (respectively $0 \leq u \leq \frac{1}{2}$ ) has zero $(s, t)$-components if $s>0$ (respectively $t>0$ ).

The argument below is reminiscent of the proof 2) of Theorem 4.9 ii).

The $d u$-part of the curvature $\left(\nabla_{\mathcal{B}, \mathcal{G}}\right)^{2}$ is given by

$$
\sum_{s, t \geq 0}(1-u)^{s} u^{t} d u \wedge\left(-(s+1) \mathcal{B}_{s+1, t}+(t+1) \mathcal{B}_{s, t+1}-(s+t+1) \mathbf{d} G_{s+1, t+1}\right.
$$




$$
\left.+\sum_{s^{\prime}+s^{\prime \prime}=s, t^{\prime}+t^{\prime \prime}=t}\left(s^{\prime}+t^{\prime}+1\right) G_{s^{\prime}+1, t^{\prime}+1} \mathcal{B}_{s^{\prime \prime}, t^{\prime \prime}}\right) .
$$

We set $G_{s, t}=0$ if $s<1$ or $t<1$, and rewrite this as a sum of $(s, t)$-components:

$$
(\underline{158})+(\underline{159})=\sum_{s, t}(1-u)^{s-1} u^{t-1} d u \wedge\left(u P_{s, t}+Q_{s, t}\right)
$$

where we set

$$
\begin{aligned}
P_{s, t} & :=(s+t)\left(-\mathcal{B}_{s, t}+\partial^{\prime} G_{s+1, t}-\partial^{\prime \prime} G_{s, t+1}\right), \\
Q_{s, t} & :=t \mathcal{B}_{s, t}-(s+t) \partial^{\prime} G_{s+1, t}+\sum_{s^{\prime}+s^{\prime \prime}=s, t^{\prime}+t^{\prime \prime}=t}\left(s^{\prime}+t^{\prime}-1\right) G_{s^{\prime}, t^{\prime}} \mathcal{B}_{s^{\prime \prime}, t^{\prime \prime}}
\end{aligned}
$$

Now we have two conditions:

$F$ : For each $(s, t)$ such that $s>0$, the integral of (160) over $\frac{1}{2} \leq u \leq 1$ is zero.

$\bar{F}$ : For each $(s, t)$ such that $t>0$, the integral of (160) over $0 \leq u \leq \frac{1}{2}$ is zero.

Let us assume first that $s, t>0$. Set $a_{s, t}:=\int_{1 / 2}^{1}(1-u)^{s-1} u^{t-1} d u>0$. Then the conditions $F$ and $\bar{F}$ are equivalent to the following equations on $P_{s, t}, Q_{s, t}$ :

$$
\begin{aligned}
& a_{s, t+1} P_{s, t}+a_{s, t} Q_{s, t}=0, \\
& a_{t+1, s} P_{s, t}+a_{t, s} Q_{s, t}=0 .
\end{aligned}
$$

Observe that $a_{s, t+1} / a_{s, t}>1 / 2$. Indeed, the integrand $(1-u)^{s-1} u^{t}$ of $a_{s, t+1}$ is obtained by multiplying the integrand $(1-u)^{s-1} u^{t-1}$ of $a_{s, t}$ by $u$, and $u>1 / 2$ on the integration interval. Similarly, $a_{t+1, s} / a_{t, s}<1 / 2$. Therefore system (162) has a unique solution:

$$
P_{s, t}=Q_{s, t}=0 \quad \text { if } s, t>0 .
$$

If $t=0$, then $Q_{s, 0}=0$. If $s=0$, then $P_{0, t}=-Q_{0, t}$. So the claim is evident in both cases.

Thanks to the Griffiths conditions for the connection $\mathbf{d}+\mathcal{B}$ and Proposition [5.14, the $d u$ part of the curvature of $\nabla_{\mathcal{B}, \mathcal{G}}$ is zero. In particular we have $P_{s, t}=0$, which just means that

$$
\mathcal{B}_{s, t}=\partial^{\prime} G_{s+1, t}-\partial^{\prime \prime} G_{s, t+1} \quad \text { where } s>0 \text { or } t>0 .
$$

This plus $g_{0,0}=\mathcal{B}_{0,0}$ proves (153). The horisontal part of the connection is zero by the very definition. Since the $d u$ part of the curvature of $\nabla_{\mathcal{B}, \mathcal{G}}$ is zero, the connection $\nabla_{\mathcal{B}, \mathcal{G}}$ is flat. Then (154) follows from Proposition 5.4. Theorem 5.7 is proved.

Explicit differential equations. Let $\psi_{s, t}=2^{-s-t}\left(\mathbf{d}^{\mathbb{C}} G\right)_{s, t}$ if $s+t>0$, and $\psi_{0,0}=g_{0,0}$. We decompose $\psi_{s, t}$ into the $(1,0)$ and $(0,1)$-components: $\psi_{s, t}=\psi_{s, t}^{\prime}+\psi_{s, t}^{\prime \prime}$. Then we have:

$$
\begin{aligned}
& s \partial^{\prime} G_{s+1, t}=\sum_{s^{\prime}+s^{\prime \prime}=s, t^{\prime}+t^{\prime \prime}=t}\left(s^{\prime}+t^{\prime}-1\right)\left[G_{s^{\prime}, t^{\prime}}, \psi_{s^{\prime \prime}, t^{\prime \prime}}^{\prime}\right], \\
& t \partial^{\prime \prime} G_{s, t+1}=\sum_{s^{\prime}+s^{\prime \prime}=s,}\left(s^{\prime}+t^{\prime}-1\right)\left[G_{s^{\prime}, t^{\prime}}, \psi_{s^{\prime \prime}, t^{\prime \prime}}^{\prime \prime}\right], \\
& \partial \bar{\partial} G_{1,1}=g_{0,0} \wedge g_{0,0} .
\end{aligned}
$$


Indeed, substituting (164) and $\mathcal{B}_{0,0}=g_{0,0}$ to (161) and using $Q_{s, t}=0$, see (163), we get the first two differential equations. The last one follows from the flatness of the horisontal part of the connection. Notice that equations (165) impose no conditions on $\partial^{\prime} G_{1, t}$ and $\partial^{\prime \prime} G_{s, 1}$ for $s, t>1$.

Lemma 5.15. Maurer-Cartan equation (142) is equivalent to differential equations (165).

Proof. Differential equations (165) just mean that the twistor connection $\nabla_{\mathcal{G}}$ is flat. By Proposition 5.4, this is equivalent to Maurer-Cartan equation $\delta \mathcal{G}+\mathcal{G} \wedge \mathcal{G}=0$, see (142).

\subsection{Conclusions}

Definition 5.16. Given a complex manifold $X$, a twistor connection is a

- $\mathbb{C}^{*} \times \mathbb{C}^{*}$-equivariant connections on $X \times \mathbb{C}^{2}$ whose restriction to $X \times\{z+w=1\}$ is flat.

Definition 5.16 is equivalent to Definition 5.5 thanks to the following Lemma.

Lemma 5.17. A twistor connection has a unique representative in its gauge class, given by

$$
\nabla_{\mathcal{G}}=\mathbf{d}+g_{0,0}+\sum_{s, t \geq 0} z^{s} w^{t}\left((s+t+1) G_{s+1, t+1}(z d w-w d z)+\left(w \partial^{\prime}-z \partial^{\prime \prime}\right) G_{s+1, t+1}\right) .
$$

Proof. A $\mathbb{C}^{*} \times \mathbb{C}^{*}$-equivariant connection looks as follows:

$$
\nabla=\mathbf{d}+\sum_{s, t \geq 0}\left(z^{s-1} w^{t} A_{s, t}^{\prime} d z+z^{s} w^{t-1} A_{s, t}^{\prime \prime} d w\right)+\sum_{s, t \geq 0} z^{s} w^{t} \mathcal{B}_{s, t} .
$$

Using a gauge transformation in the fibers $\mathbb{C}^{2}$, it can be uniquely transformed into

$$
\nabla=\mathbf{d}+\sum_{s, t \geq 0} z^{s-1} w^{t-1}(s+t-1) G_{s, t}(z d w-w d z)+\sum_{s, t \geq 0} z^{s} w^{t} \mathcal{B}_{s, t} .
$$

The $d u$-part of the curvature of its restriction to $X \times \mathbb{C}$ is given by

$$
\sum_{s, t}(1-u)^{s-1} u^{t-1} d u \wedge\left(u P_{s, t}+Q_{s, t}\right)
$$

where the $P_{s, t}$ and $Q_{s, t}$ are given by (161). Just as in the proof of Lemma 4.14, this implies that $P_{s, t}=0$. Indeed, the $P_{s, t}$ is the leading coefficient of the polynomial in $u$ giving the $(-s,-t)$-part. This immediately implies that $Q_{s, t}=0$. The $P_{s, t}=0$ just means that

$$
\sum_{s, t \geq 0} z^{s} w^{t} \mathcal{B}_{s, t}=\mathcal{B}_{0,0}+\sum_{s, t \geq 0} z^{s} w^{t}\left(w \partial^{\prime}-z \partial^{\prime \prime}\right) G_{s+1, t+1}
$$

It remains to set $g_{0,0}=\mathcal{B}_{0,0}$.

Using Lemma 5.17 one can restate Theorem 5.7 as follows.

Theorem 5.18. The tensor category of variations of mixed Hodge structures on a complex manifold $X$ is equivalent to the tensor category of twistor connections on $X$.

Deligne's operator $\mathrm{d}$ is the monodromy of the twistor connection over the triangle with vertices $\{0,0\},\{0,1\},\{1,0\}$ in the twistor plane $\mathbb{C}^{2}$, while the Green operator $(s+t+1) G_{s+1, t+1}$ is the $(s+1, t+1)$-homogeneous component of the curvature form in the twistor plane. 


\subsection{Variations of real mixed Hodge structures.}

The category of variations of real mixed Hodge structures as the category of $\operatorname{Gal}(\mathbb{C} / \mathbb{R})$-equivariant objects in the category of variations of mixed Hodge structures. Here is an elaborate description.

Definition 5.19. A variation of mixed $\mathbb{R}$-Hodge structures over a complex manifold $X$ is given by a real local system $(\mathcal{L}, \nabla)$ on $X$ equipped with the following data:

- An increasing weight filtration $W \cdot \mathcal{L}$ on the local system $\mathcal{L}$,

- A decreasing Hodge filtration $F^{\bullet}$ on the holomorphic vector bundle $\mathcal{L}_{\mathcal{O}}:=\mathcal{L} \otimes_{\mathbb{R}} \mathcal{O}_{X}$,

satisfying the following conditions:

- The two filtrations induce at each fiber a mixed $\mathbb{R}$-Hodge structure,

- The Griffiths transversality condition: $\nabla^{1,0}\left(F^{p}\right) \subset F^{p-1} \otimes \Omega_{M}^{1}$.

A Green datum on a variation of real Hodge structures $\mathcal{V}$ is a Maurer-Cartan element:

$$
\mathcal{G} \in \mathcal{C}_{\mathcal{H}, \mathbb{R}}^{1}(\operatorname{End} \mathcal{V}), \quad \delta \mathcal{G}+\mathcal{G} \wedge \mathcal{G}=0
$$

Explicitly, $\mathcal{G}$ is given by the data $\left(G_{p, q}, g_{0,0}\right)$ as in Lemma 5.3, satisfying the reality condition:

$$
\bar{G}_{p, q}=-G_{p, q} \text {, i.e. } \bar{G}=-G, \quad \text { and } \overline{g_{0,0}}=g_{0,0} \cdot
$$

Equivalently, a real twistor connection is a twistor connection which is invariant under the involution $c \circ \sigma$, where $\sigma:(z, w) \longmapsto(\bar{w}, \bar{z})$, and $c$ is the complex conjugation.

The category of real twistor connections on $X$ is equivalent to the category variations of real mixed Hodge structures.

The complex conjugation interchanges the first two differential equations in (165).

\subsection{DG generalizations}

Recall that a DG-module over a DG Lie algebra is a module over a Lie algebra in the category of complexes rather then vector spaces. So a DG module over a DG Lie algebra $\left(L_{\bullet}, \partial\right)$ is a complex $M_{\bullet}$ plus an action of the graded Lie algebra $L_{\bullet}$ on the graded object $M_{\bullet}$ given by a map of complexes $\mu: L_{\bullet} \otimes M_{\bullet} \rightarrow M_{\bullet}$.

Given two DG-modules $M_{\bullet}$ and $N_{\bullet}, \operatorname{Hom}_{L_{\bullet}}^{\bullet}\left(M_{\bullet}, N_{\bullet}\right)$ is a complex, consisting of elements of the complex $\operatorname{Hom}^{\bullet}\left(M_{\bullet}, N_{\bullet}\right)$ commuting with the action of $L_{\bullet}$. Taking $H^{0}$ 's of these Homcomplexes we arrive at the homotopy category of $D G$-modules. Here the morphisms are morphisms of $L_{\bullet}$-modules $M_{\bullet} \rightarrow N_{\bullet}$ commuting with the differentials in $M_{\bullet}$ and $N_{\bullet}$, up to homotopies given by morphisms of $L_{\bullet}$-modules $M_{\bullet} \rightarrow N_{\bullet}[-1]$. Localizing by the quasiisomorphisms, we get the derived category $\mathcal{D}\left(L_{\bullet}\right)$. One easily translates this to the world of DG Lie coalgebras.

Recall the DG Lie coalgebra $\mathcal{L}_{\mathcal{H} ; X}^{*}$ in the pure category $\operatorname{Hod}_{X}$ defined in Section 1.9.

Definition 5.20. The DG Hodge category $\mathcal{G}_{\mathcal{H}}^{*}(X)$ of a regular complex projective variety $X$ is the derived category $\mathcal{D}\left(\mathcal{L}_{\mathcal{H} ; X}^{*}\right)$ of $D G$ comodules over the $D G$ coalgebra $\mathcal{L}_{\mathcal{H} ; X}^{*}$ in the pure category $\operatorname{Hod}_{X}$. 
The precise form of Conjecture 1.11 is

Conjecture 5.21. The category of smooth complexes of real Hodge sheaves on $X$ is equivalent to the derived category $\mathcal{D}\left(\mathcal{L}_{\mathcal{H} ; X}^{*}\right)$.

Let us describe DG comodules over the DG Lie coalgebra $\mathcal{L}_{\mathcal{H} ; X}^{*}$. Consider the following data:

- A bounded complex $\mathcal{V}=\left\{\ldots \longrightarrow \mathcal{V}^{1} \stackrel{\partial}{\longrightarrow} \mathcal{V}^{2} \stackrel{\partial}{\longrightarrow} \mathcal{V}^{3} \stackrel{\partial}{\longrightarrow} \mathcal{V}^{4} \stackrel{\partial}{\longrightarrow} \ldots\right\}$ in $\operatorname{Hod}_{X}$.

- A Maurer-Cartan element:

$$
\mathcal{G} \in \mathcal{C}_{\mathcal{H}}^{1}(\text { End } \mathcal{V}) \quad \text { such that } \quad \delta \mathcal{G}+\mathcal{G} \wedge \mathcal{G}=0
$$

defined modulo elements of type $\delta B+[B, \mathcal{G}]$, where $B \in \mathcal{C}_{\mathcal{H}}^{0}($ End $\mathcal{V})$.

Here End $\mathcal{V}=\mathcal{V}^{\vee} \otimes \mathcal{V}$ is a complex in $\operatorname{Hod}_{X}$. The complex $\mathcal{C}_{\mathcal{H}}^{*}($ End $\mathcal{V})$ is the total complex of the bicomplex $\mathcal{C}_{\mathcal{H}}^{\bullet}(\operatorname{End} \mathcal{V})$. So an element (169) includes a function on $X$ with values in $\operatorname{End}^{0} \mathcal{V}$, a 1 -form with values in $\mathrm{End}^{-1} \mathcal{V}$, etc. Thanks to Theorem 5.7 it provides the cohomology $H^{*}(\mathcal{V})$ with a variation of $\mathbb{R}-$ MHS's data.

Lemma 5.22. (i) DG comodules over the DG Lie coalgebra $\mathcal{C}_{\mathcal{H}}^{*}($ End $\mathcal{V})$ are the same thing as Maurer-Cartan elements (169).

(ii) Homotopy equivalent $D G$ comodules correspond to the equivalent Maurer-Cartan elements.

Proof. (i) Neglecting the differential, the graded Lie coalgebra $\mathcal{L}_{\mathcal{H}: X}^{*}$ is a free Lie coalgebra cogenerated by the graded object $\mathcal{D}_{X}[1]$ in the category $\operatorname{Hod}_{X}$. Therefore a comodule over $\mathcal{L}_{\mathcal{H} ; X}^{*}$ is determined by the coaction of the cogenerators. The latter is given by a graded object $\mathcal{V}$ plus a linear map $\mathcal{V} \longrightarrow \mathcal{V} \otimes_{\mathbb{R}} \mathcal{D}_{X}[1]$. It can be interpreted as a degree zero element in $\mathcal{D}_{X}[1] \otimes_{\mathbb{R}}$ End $\mathcal{V}$, which is the same as an element $\mathcal{G} \in \mathcal{C}_{\mathcal{H}}^{1}($ End $\mathcal{V})$.

ii) Similarly a homotopy is given by an element $\mathcal{V} \longrightarrow \mathcal{V} \otimes_{\mathbb{R}} \mathcal{D}_{X}$, which can be interpreted as an element $B \in \mathcal{C}_{\mathcal{H}}^{0}($ End $\mathcal{V})$.

Assume that $L_{k}=0$ for $k>0$. Then subcategory of DG-modules concentrated in the degree zero is canonically identified with the category of modules over the Lie algebra $H_{0}\left(L_{\bullet}\right)$. Indeed, given such a module $M_{0}, L_{k}$ where $k \neq 0$ acts on it trivially since $M_{0}$ a graded module over a graded Lie algebra. Further, since the action map $\mu$ is a map of complexes, $\partial\left(L_{-1}\right)$ acts on $M_{0}$ trivially.

There is a similar picture for DG comodules over a DG Lie coalgebra concentrated in degrees $\geq 0$, which we use below.

Proof of Lemma 5.6. Follows immediately from Lemma 5.22 .

A $D G$-connection on $X$ is a first order linear differential operator $\mathbf{d}+\mathcal{A}$ where $\mathcal{A}$ is an End $\mathcal{V}$-valued differential form on $X$ of total degree 1 . A DG-connection is flat if $(\mathbf{d}+\mathcal{A})^{2}=0$.

Definition 5.23. Let $\mathcal{V}$ be a variation of real Hodge structures on $X$, and $\mathcal{G} \in \mathcal{C}_{\mathcal{H}}^{1}($ End $\mathcal{V})$. The twistor DG-connection of $\mathcal{G}$ is a $D G$-connection on $X$ given by $\nabla_{\mathcal{G}}:=\mathbf{d}+\gamma(\mathcal{G})$.

Proposition 5.24. A form $\mathcal{G} \in \mathcal{C}_{\mathcal{H}}^{1}($ End $\mathcal{V})$ satisfies the Maurer-Cartan equation $\delta \mathcal{G}+\mathcal{G} \wedge \mathcal{G}=0$ if and only if the twistor DG-connection $\nabla_{\mathcal{G}}$ is flat.

Proof. Follows immediately from Theorem 1.9. 


\section{DG Lie algebra $\mathcal{C}_{H, S}$ and $L_{\infty}$-algebra of plane decorated trees}

Let $H^{\vee}$ be a finite dimensional symplectic vector space, and $S$ a finite set. Set

$$
\mathrm{V}_{H, S}^{\vee}:=H^{\vee} \oplus \mathbb{C}[S] .
$$

Let $\mathcal{C}_{H, S}^{\vee}:=\mathcal{C} \mathrm{T}\left(\mathrm{V}_{H, S}^{\vee}\right)$ be the cyclic envelope of the tensor algebra of $\mathrm{V}_{H, S}^{\vee}$. In Section 6.1 we define a Lie coalgebra structure on $\mathcal{C}_{H, S}^{\vee}$. The coproduct generalizes the coproduct for the dihedral Lie coalgebras from [G4 - the latter corresponds to the $H^{\vee}=0$ case. The Lie coalgebra structure on $\mathcal{C}_{H, S}^{\vee}$ provides the dual vector space $\mathcal{C}_{H, S}$ with a Lie algebra structure. The Lie algebra $\mathcal{C}_{H, S}$ plays a key role in our story. We give a different interpretation of the Lie algebra $\mathcal{C}_{H, S}$ in Section 8 .

In Section 6.2 we define an $L_{\infty}$-coalgebra $\left(\mathcal{T}_{H, S}^{\vee}, \bullet[1], \partial\right)$ of the plane $\mathrm{V}_{H, S}^{\vee}$-decorated trees.

Its dual is an $L_{\infty}$-algebra $\mathcal{T}_{H, S}^{\bullet}[-1]$ of plane $\mathrm{V}_{H, S}$-decorated trees.

The standard cochain complex of the $L_{\infty}$-algebra $\mathcal{T}_{H, S}^{\bullet}[-1]$ is identified with a commutative differential graded algebra $\mathcal{F}_{H, S}^{\vee, \bullet}$ of plane $\mathrm{V}_{H, S}^{\vee}$-decorated forests:

$$
S^{\bullet}\left(\mathcal{T}_{H, S}^{\vee, \bullet}\right)=\mathcal{F}_{H, S}^{\vee, \bullet}
$$

The $L_{\infty}$-coalgebra structure on $\mathcal{T}_{H, S}^{\vee, \bullet}[1]$ generalises both the Kontsevich-Boardman differential $\partial_{\Delta}$ in the graph complex, and the differential defined in G5. The differential $\partial_{\Delta}$ is indentified with the differential on the $\mathcal{T}_{H, S}^{\vee, \bullet}[1]$. Our definition was suggested by computation of the differential of the Hodge correlator in Theorem 7.11 ,

The sum over all plane trivalent trees with a given decoration gives rise to an injective map

$$
F: \mathcal{C}_{H, S}^{\vee} \hookrightarrow \mathcal{T}_{H, S}^{\vee, 1}[1] .
$$

The $L_{\infty}$-algebra $\mathcal{T}_{H, S}^{\bullet}[-1]$ with the differential $\partial_{\Delta}$ is a resolution of the Lie algebra $\mathcal{C}_{H, S}$. So

$$
\mathcal{C}_{H, S}=H_{0}\left(\mathcal{T}_{H, S}^{\bullet}[-1]\right)
$$

\subsection{A Lie algebra structure on $\mathcal{C}_{H, S}$}

Let us define a cobracket $\delta: \mathcal{C}_{H, S}^{\vee} \longrightarrow \Lambda^{2} \mathcal{C}_{H, S}^{\vee}$. It is a sum of two maps:

$$
\delta=\delta_{\mathrm{Cas}}+\delta_{S}
$$

Let us define these maps. We picture a cyclic word $W$ on an oriented circle.

(i) The map $\delta_{\text {Cas }}$. Cut two different arcs of the circle. We get two semicircles. Make an oriented circle out of each of them. Each of them comes with a special point on it, obtained by gluing the ends of the semicircle. Decorate the special point of the first circle by $\alpha_{k}$, and the special point of the second circle by $\alpha_{k}^{\vee}$, take the sum over $k$, and put the sign. This is illustrated on Fig 8. The map $\delta_{\text {Cas }}$ is obtained by taking the sum over all possible cuts.

Observe that the wedge product comes with the plus sign, and changing the order of the cuts (and hence the order of the terms in the wedge product) we do not change it, since if $\left\{\alpha_{k}\right\}$, $\left\{\alpha_{k}^{\vee}\right\}$ is a basis and its symplectic dual, then the same is true for $\left\{-\alpha_{k}^{\vee}\right\},\left\{\alpha_{k}\right\}$.

(ii) The map $\delta_{S}$. Cut the circle at an arc and at an $S$-decorated point, which is not at the end of the arc, and make two circles as before. Their special points inherit an $S$-decoration from the $S$-decorated point, see Fig 9, Make the sum over all possible cuts.

This time the order of the terms in the wedge product matters. We put the semicircle which, going according to the orientation of the circle, has the $S$-cut at the end as the first term. 


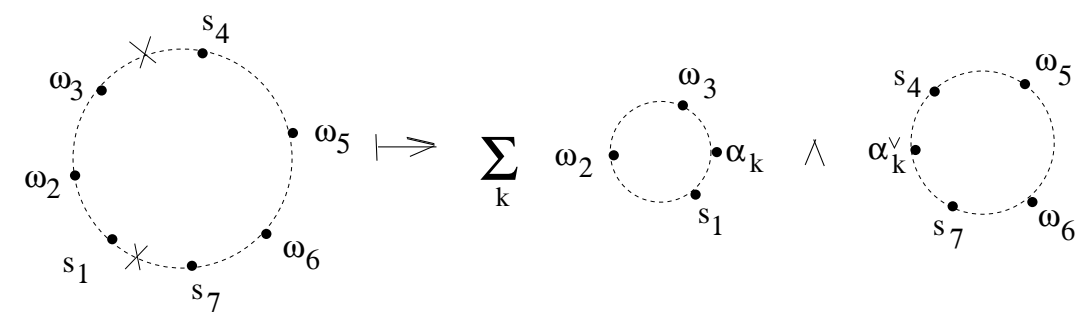

Figure 8: The component $\delta_{\text {Cas }}$ of the differential.
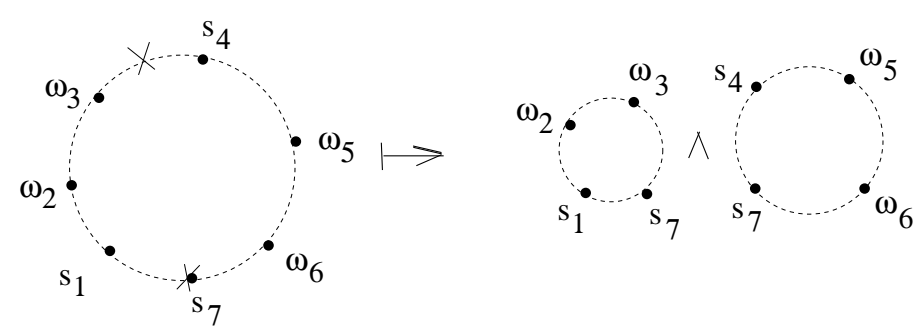

Figure 9: The component $\delta_{S}$ of the differential.

Examples. One has

$$
\begin{gathered}
\delta \mathcal{C}\left(\left\{s_{0}\right\} \otimes\left\{s_{1}\right\}\right)=\mathcal{C}\left(\left\{s_{0}\right\} \otimes \alpha_{k}\right) \wedge \mathcal{C}\left(\alpha_{k}^{\vee} \otimes\left\{s_{1}\right\}\right) . \\
\delta \mathcal{C}\left(\left\{s_{0}\right\} \otimes\left\{s_{1}\right\} \otimes\left\{s_{2}\right\}\right)= \\
\mathrm{Cycle}_{0,1,2}\left(\mathcal{C}\left(\left\{s_{0}\right\} \otimes\left\{s_{1}\right\} \otimes \alpha_{k}\right) \wedge \mathcal{C}\left(\alpha_{k}^{\vee} \otimes\left\{s_{2}\right\}\right)+\mathcal{C}\left(\left\{s_{0}\right\} \otimes\left\{s_{1}\right\}\right) \wedge \mathcal{C}\left(\left\{s_{1}\right\} \otimes\left\{s_{2}\right\}\right) .\right.
\end{gathered}
$$

where Cycle $_{0,1,2}$ means the cyclic sum. See Fig 10 illustrating the last formula.

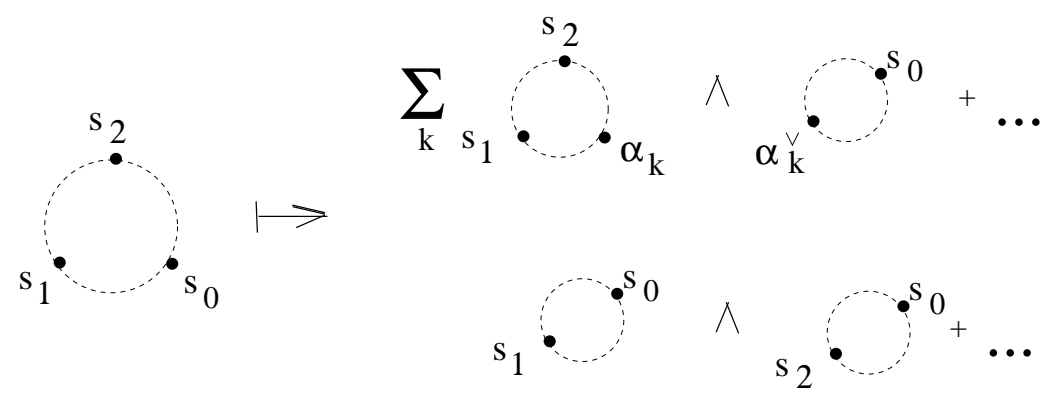

Figure 10: The coproduct of $\mathcal{C}\left(\left\{s_{0}\right\} \otimes\left\{s_{1}\right\} \otimes\left\{s_{2}\right\}\right)$.

Proposition 6.1. One has $\delta^{2}=0$.

Proposition 6.1 is deduced from the construction of the $L_{\infty}$-coalgebra of plane decorated trees, see the end of Section 6 .

\subsection{A commutative differential graded algebra of plane decorated forests}

We start from a background on DG Lie coalgebras, $L_{\infty}$-coalgebras, decorated trees and forests. 
DG Lie coalgebras. Let $\mathcal{L}^{\bullet}$ be a DG Lie coalgebra with a differential $d$. Its cobracet $\delta$ : $\mathcal{L}^{\bullet} \longrightarrow \Lambda^{2} \mathcal{L}^{\bullet}$ is a map of complexes: $\delta d=d \delta$. The map $\delta$ is understood as another differential

$$
\delta: \mathcal{L}^{\bullet}[-1] \longrightarrow S^{2}\left(\mathcal{L}^{\bullet}[-1]\right)
$$

We alter the signs of $\delta$, so that $\delta d+d \delta=0$. Then the symmetric algebra $S^{\bullet}\left(\mathcal{L}^{\bullet}[-1]\right)$ is a commutative DGA with a differential $\partial$, given by the map $d+\delta$ on the space of cogenerators $\mathcal{L}^{\bullet}[-1]$. Conversely, consider a commutative DGA $S^{\bullet}\left(\mathcal{L}^{\bullet}[-1]\right)$ with a differential $\partial$, such that

$$
\partial: \mathcal{L}^{\bullet}[-1] \longrightarrow \mathcal{L}^{\bullet}[-1] \oplus S^{2}\left(\mathcal{L}^{\bullet}[-1]\right) .
$$

Then there is DG Lie coalgebra structure on $\mathcal{L}^{\bullet}$ defined as follows. Write the map (173) as $\partial=d+\delta$, where $d$ and $\delta$ are the two natural components, where $d$ is the one preserving $\mathcal{L}^{\bullet}[-1]$. Then $d$ is a differential on $\mathcal{L}^{\bullet}$, and $\delta$ is a Lie coalgebra coproduct on $\mathcal{L}^{\bullet}$.

$L_{\infty}$-coalgebras. Generaliseing the last construction, a graded $L_{\infty}$-coalgebra structure on a graded vector space $\mathcal{L}^{\bullet}$ is given by a differential $\partial$ of the graded commutative algebra $S^{\bullet}\left(\mathcal{L}^{\bullet}[-1]\right)$, $\partial^{2}=0$. It is determined by its action on the cogenerators:

$$
\partial: \mathcal{L}^{\bullet}[-1] \longrightarrow S^{\bullet}\left(\mathcal{L}^{\bullet}[-1]\right)
$$

Write it as a sum of the $S^{\bullet}$-components: $\partial=\sum_{i>1} \partial_{i}$. The component $\partial_{1}: \mathcal{L}^{\bullet}[-1] \longrightarrow \mathcal{L}^{\bullet}[-1]$ is a differential. If $\partial_{m}=0$ for $m>2$ then it is a DG Lie coalgebra with the coproduct $\partial_{2}$.

Trees, forests, decorations. Below a plane tree is a tree with internal vertices of valency $\geq 3$. A tree may have no internal vertices, i.e. just two external vertices. A plane forest is a disjoint union of plane trees. An $R$-decorated plane forest is a plane forest decorated by a set $R$. If $F=T_{1} \cup \ldots \cup T_{k}$ is a forest presented as a union of trees $T_{i}$, its orientation torsor is the product of the tree orientation torsors: or $_{F}=$ or $_{T_{1}} \otimes \ldots \otimes$ or $_{T_{k}}$.

Decorations by cyclic words. A decoration of a tree $T$ by vectors of a vector space $V$ gives rise to a decoration of $T$ by an element $\mathcal{C} \mathrm{T}(V)$ : if the external edges are decorated clockwise by the vectors $v_{1}, \ldots, v_{n}$, the resulting decoration is a cyclic word $W:=\mathcal{C}\left(v_{1} \otimes \ldots \otimes v_{n}\right)$. We say then that $T$ is decorated by the cyclic word $W$. Different decorations of external edges of $T$ by vectors $v_{i}$ can lead to the same decoration by a cyclic word $W$.

Definition 6.2. A decoration of a forest is the symmetric product $W_{T_{1}} \circ \ldots \circ W_{T_{n}}$ of cyclic words $W_{T_{i}}$ decorating its connected components $T_{i}$.

The graded space of decorated trees. Let $\mathcal{T}_{H, S}^{\vee, \bullet}$ be the graded $\mathbb{C}$-vector space generated by triples $\left(T, W ; \mathrm{Or}_{T}\right)$ where $T$ is a plane tree, $\mathrm{Or}_{T}$ is an element of the orientation torsor of $T$, and $W$ is a $\mathrm{V}_{H, S}^{\vee}$-decoration of $T$. The relations between the generators are the following:

$$
\left(T, W ; \mathrm{Or}_{T}\right)=-\left(T, W ;-\mathrm{Or}_{T}\right), \quad\left(T, \lambda W_{1}+\mu W_{2} ; \mathrm{Or}_{T}\right)=\lambda\left(T, W_{1} ; \mathrm{Or}_{T}\right)+\mu\left(T, W_{2} ; \mathrm{Or}_{T}\right) .
$$

The degree is defined as follows, where $v$ runs through the set of all internal vertices of $T$ :

$$
\operatorname{deg}\left(T, W ; \operatorname{Or}_{T}\right):=1+\sum_{v}(\operatorname{val}(v)-3) .
$$

The extra +1 in the formula for the degree is necessary to have a differetial $\partial$, defined below. 
The graded commutative algebra of decorated forests. Consider the symmetric algebra of the graded space of $\mathcal{T}_{H, S}^{\vee, \bullet}$ of $\mathrm{V}_{H, S}^{\vee}$-decorated plane trees:

$$
\mathcal{F}_{H, S}^{\vee, \bullet}:=S^{\bullet}\left(\mathcal{T}_{H, S}^{\vee, \bullet}\right)
$$

It is a graded commutative algebra. The vector space $\mathcal{F}_{H, S}^{\vee, \bullet}$ is spanned by oriented forests decorated by symmetric products of cyclic words in $\mathrm{V}_{H, S}^{\vee}$. The grading is given by the degree

$$
\operatorname{deg}\left(F, W ; \operatorname{Or}_{F}\right):=\sum_{\text {vertices } v \text { of } F}(\operatorname{val}(v)-3)+\pi_{0}(F) .
$$

The product in $\mathcal{F}_{H, S}^{\vee, \bullet}$ is described on the generators by

$$
\left(F_{1}, W_{1} ; \mathrm{Or}_{T_{1}}\right) *\left(F_{2}, W_{2} ; \mathrm{Or}_{T_{2}}\right)=\left(F_{1} \cup F_{2}, W_{1} \circ W_{2} ; \mathrm{Or}_{T_{1}} \wedge \mathrm{Or}_{T_{2}}\right)
$$

Commutative DGA $\mathcal{F}_{H, S}^{\vee, \bullet}$ of plane decorated forests. Let us define a differential

$$
\partial: \mathcal{F}_{H, S}^{\vee, \bullet} \rightarrow \mathcal{F}_{H, S}^{\vee, \bullet+1}
$$

When $H^{\vee}=0$ it is the differential defined in G5. We define it on trees, and then extend by the Leibniz rule. It has three components:

$$
\partial=\partial_{\Delta}+\partial_{\text {Cas }}+\partial_{\mathrm{S}}
$$

(i) The map $\partial_{\Delta}$. Let $\left(T, W_{T} ; \mathrm{Or}_{T}\right)$ be a generator and $E$ an internal edge of $T$, see Fig 11 . Let $T / E$ be the tree obtained by contraction of the edge $E$. So it has one less edge, and one less vertex. The orientation $\mathrm{Or}_{T}$ of the tree $T$ induces an orientation $\mathrm{Or}_{T / E}$ of the tree $T / E$. Namely, if $\mathrm{Or}_{T}=E \wedge E_{1} \wedge E_{2} \wedge \ldots$ then $\mathrm{Or}_{T / E}=E_{1} \wedge E_{2} \wedge \ldots$. Constructing an internal edge we do not touch the decoration. Set

$$
\partial_{\Delta}\left(T, W_{T} ; \mathrm{Or}_{T}\right):=\sum_{E: \text { internal edges of } T}\left(T / E, W_{T} ; \mathrm{Or}_{T / E}\right) .
$$

It is (a decorated version of) the graph complex differential of Boardman and Kontsevich.

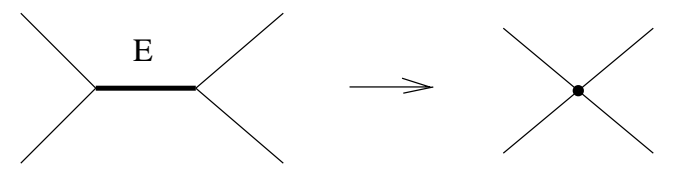

Figure 11: $\partial_{\Delta}$ : The $\delta_{X}$-term for an internal edge $E$.

(ii) The map $\partial_{\text {Cas }}$. Let $E$ be an edge of $T$. Cut the tree $T$ along the edge $E$, getting two trees, $T_{1}$ and $T_{2}$, see Fig 12 and Fig [16. Choose their orientations $\mathrm{Or}_{T_{1}}$ and $\mathrm{Or}_{T_{2}}$ so that $\mathrm{Or}_{T}=E \wedge \mathrm{Or}_{T_{1}} \wedge \mathrm{Or}_{T_{2}}$. The trees $T_{i}$ inherit partial decorations by the (non-cyclic!) words $W_{i}^{\prime}$, so that $W_{T}=\mathcal{C}\left(W_{1}^{\prime} \otimes W_{2}^{\prime}\right)$. Denote by $E_{1}$ and $E_{2}$ the new external edges of the trees $T_{1}$ and $T_{2}$ obtained by cutting $E$.

Below we use the identity (Casimir) element Id $: H \rightarrow H$. We write it as follows. Choose a basis $\left\{\alpha_{k}\right\}$ of $H$. Denote by $\left\{\alpha_{k}^{\vee}\right\}$ the dual basis: $\left(\alpha_{k}, \alpha_{l}^{\vee}\right)=\delta_{k l}$. Then $\operatorname{Id}=\sum_{k} \alpha_{k}^{\vee} \otimes \alpha_{k}$. 


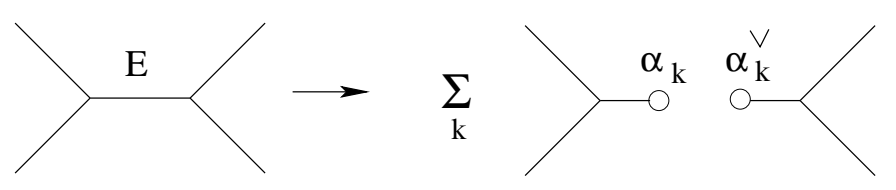

Figure 12: The Casimir map $\partial_{\text {Cas }}$ for an internal edge $E$.

We decorate $E_{1}$ by $\alpha_{k}, E_{2}$ by $\alpha_{k}^{\vee}$, getting a decoration $\mathcal{C}\left(W_{1} \otimes \alpha_{k}\right)$ of the tree $T_{1}$, and a decoration $\mathcal{C}\left(\alpha_{k}^{\vee} \otimes W_{2}\right)$ of the tree $T_{2}$. Then

$$
\partial_{\mathrm{Cas}}\left(T, W_{T} ; \mathrm{Or}_{T}\right):=\sum_{E} \sum_{k}\left(T_{1}, \mathcal{C}\left(W_{1}^{\prime} \otimes \alpha_{k}\right) ; \mathrm{Or}_{T_{1}}\right) \wedge\left(T_{2}, \mathcal{C}\left(\alpha_{k}^{\vee} \otimes W_{2}^{\prime}\right) ; \mathrm{Or}_{T_{2}}\right)
$$

where the first sum is over all internal edges $E$ of $T$.

(iii) The map $\partial_{S}$. Let $E$ be an external $S$-decorated edge of $T$, see Fig 13, Remove it together with a little neighborhood of its vertices. If $E \neq T$, one of the vertices has the valency $v \geq 3$. The tree $T$ is replaced by $v-1$ trees $T_{1}, \ldots, T_{v-1}$ : each of the internal edges sharing the vertex with $E$ produces a new tree. Choose their orientations $\mathrm{Or}_{T_{i}}$ so that $\mathrm{Or}_{T}=E \wedge \mathrm{Or}_{T_{1}} \wedge \ldots \wedge \mathrm{Or}_{T_{v-1}}$. The decoration of the tree $T$ provides a decorations $W_{T_{i}}$ of the new trees $T_{i}$, so that the new
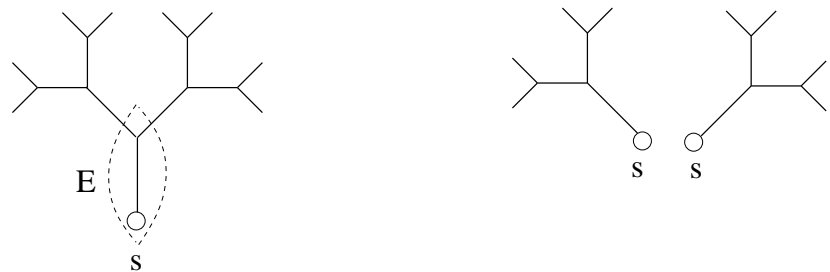

Figure 13: The map $\partial_{S}$ : contribution of an external edge $E$.

external vertex of each of the trees $T_{i}$ inherits the $S$-decoration of $E$. Set

$$
\begin{aligned}
& \frac{\partial}{\partial E}\left(T, W_{T} ; \mathrm{Or}_{T}\right)=\left(T_{1}, W_{T_{1}} ; \mathrm{Or}_{T_{1}}\right) \wedge \ldots \wedge\left(T_{v-1}, W_{T_{v-1}} ; \mathrm{Or}_{T_{v-1}}\right), \\
& \partial_{S}:=\sum_{E: S \text {-decorated }} \frac{\partial}{\partial E} .
\end{aligned}
$$

Theorem 6.3. The map $\partial=\partial_{\Delta}+\partial_{\text {Cas }}+\partial_{\mathrm{S}}$ is a differential on the graded commutative algebra $\mathcal{F}_{H, S}^{\vee, \bullet}: \partial^{2}=0$. More specifically, $\partial_{\text {Cas }}$ anticommutes with $\partial_{\Delta}+\partial_{\mathrm{S}}$, and one has

$$
\partial_{\Delta}^{2}=0, \quad \partial_{\text {Cas }}^{2}=0, \quad\left(\partial_{\Delta}+\partial_{\mathrm{S}}\right)^{2}=0 .
$$

Proof. Thanks to the term $\pi_{0}(F)$ in formula (174) for the degree, the map $\partial$ has degree +1 . Notice that without the $\pi_{0}(F)$ the map $\partial_{\text {Cas }}$ has degree 0 , and the map $\partial_{\mathrm{S}}$ is not homogeneous.

It is well known that $\partial_{\Delta}^{2}=0$ : the $\partial_{\Delta}$ is the Kontsevich-Boardman differential.

To check that $\partial_{\text {Cas }}^{2}=0$ observe that $\partial_{\text {Cas }}^{2}\left(T, W ; \mathrm{Or}_{T}\right)$ is a sum of two terms, one of whom is

$$
\sum_{E_{1}, E_{2}} \sum_{k, l}\left(T_{1}, \mathcal{C}\left(W_{1} \otimes \alpha_{k}\right) ; \mathrm{Or}_{T_{1}}\right) \wedge\left(T_{2}, \mathcal{C}\left(\alpha_{k}^{\vee} \otimes W_{2} \otimes \beta_{l}\right) ; \mathrm{Or}_{T_{2}}\right) \wedge\left(T_{3}, \mathcal{C}\left(\beta_{l}^{\vee} \otimes W_{3}\right) ; \mathrm{Or}_{T_{3}}\right)
$$




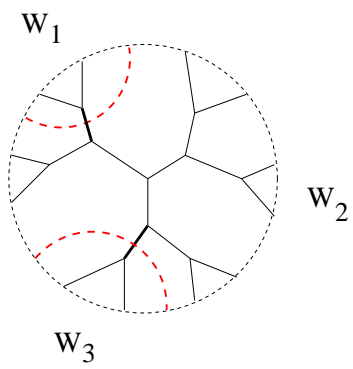

Figure 14: The Casimir map is a differential: $\partial_{\text {Cas }}^{2}=0$.

and the other has the opposite sign, see Fig 14. Here we cut the tree $T$ along the edges $E_{1}$ and $E_{2}$, shown by the thick segments. The dotted arcs show how cutting along these edges we get three new trees. Computing the signs we see that the differentials $\partial_{\text {Cas }}, \partial_{\Delta}$ anticommute.

Although $\partial_{\mathrm{S}}^{2} \neq 0$, we have $\left(\partial_{\mathrm{S}}+\partial_{\Delta}\right)^{2}=0$. To show this it is sufficient to trace the contribution of a single S-decorated external edge $E$. For any internal edge $F$ not incident to $E$, the naive operations of shrinking of the edge $F$ and removing the edge $E$ evidently commute. So taking into account the orientation torsors, we see that the $\partial_{\mathrm{S}}$-component at $E$ and the $\partial_{\Delta}$-component at $F$ anticommute. The contributions of the incident edges $E$ and $F$ are depicted on Fig 15 , Naively, $\partial_{S_{F}} \circ \partial_{S_{E}}$ gives the same result as $\partial_{\Delta_{E}} \circ \partial_{S_{F}}$. So taking into account the orientation torsor, we get $\partial_{S_{F}} \partial_{S_{E}}+\partial_{\Delta_{E}} \partial_{S_{F}}=0$.

Similarly, the maps $\partial_{\text {Cas }}, \partial_{\mathrm{S}}$ anticommute: notice that $\partial_{\text {Cas }}$ does not create new S-decorated vertices.

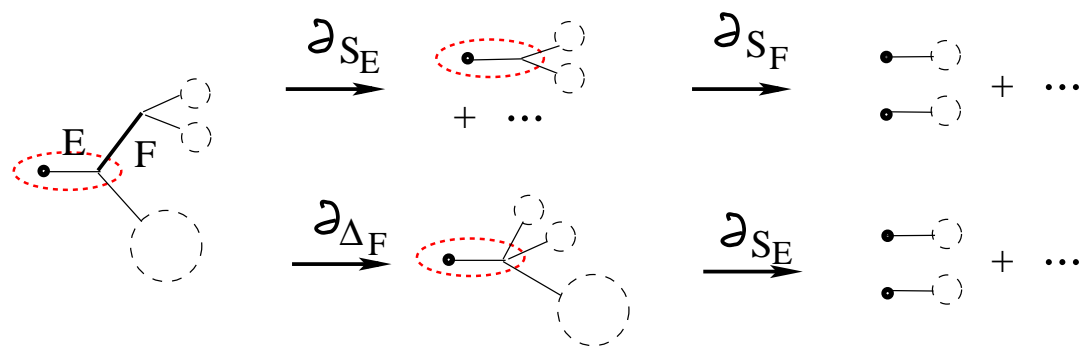

Figure 15: Illustrating $\left(\partial_{\mathrm{S}}+\partial_{\Delta}\right)^{2}=0$. The S-decorated external vertex of the edge $E$ and its descendants are the boldface vertices. We also trace contributions of the internal edge $F$ to $\partial_{\Delta}$.

$L_{\infty}$-coalgebra $\mathcal{T}_{H, S}^{\vee, \bullet}[1]$ of plane decorated trees. The map $\partial_{\Delta}$ is the component of the differential $\partial$ on the DGCom $\mathcal{F}_{H, S}^{\vee, \bullet}$ preserving the subspace $\mathcal{T}_{H, S}^{\vee}$. So we get a differential

$$
\partial_{\Delta}: \mathcal{T}_{H, S}^{\vee, \bullet} \longrightarrow \mathcal{T}_{H, S}^{\vee, \bullet}
$$

The differential $\partial$ defines a $L_{\infty}$-coproduct

$$
\partial: \mathcal{T}_{H, S}^{\vee, \bullet} \longrightarrow S^{\bullet}\left(\mathcal{T}_{H, S}^{\vee, \bullet}\right)
$$

Therefore $\mathcal{T}_{H, S}^{\vee, \bullet}[1]$ is an $L_{\infty}$-coalgebra with a differential $\partial_{\Delta}$ and a $L_{\infty}$-cobracket $\partial$. The commutative DGA $\mathcal{F}_{H, S}^{\vee, \bullet}$ is the standard cochain complex of the $L_{\infty}$-coalgebra $\mathcal{T}_{H, S}^{\vee, \bullet}[1]$. 


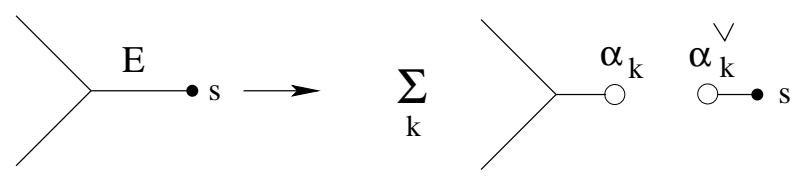

Figure 16: $\partial_{\text {Cas }}$ : Contribution of the Casimir term for an external edge $E$.

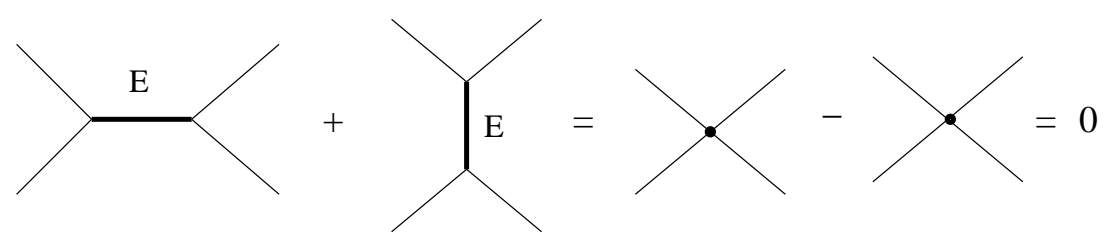

Figure 17: The two terms for the internal edges $E$ cancel each other.

The sum over plane trivalent trees map. Let us define an injective linear map

$$
F: \mathcal{C}_{H, S}^{\vee} \hookrightarrow \mathcal{T}_{H, S}^{\vee, 1}[1], \quad F(W):=\sum_{T}\left(T, W ; \mathrm{Or}_{T}\right)
$$

The sum is over all plane trivalent trees decorated by the cyclic word $W$, and $\mathrm{Or}_{T}$ is the canonical orientation of the trivalent tree $T$ corresponding to the clockwise orientation of the plane.

Lemma 6.4. i) The differential $\partial_{\Delta}$ is zero on the image of the map $F$, i.e. $\partial_{\Delta} F=0$.

ii) The map $F$ commutes with the differentials: $\partial F=F \delta$.

Proof. i) Let $E$ be an internal edge. There are exactly two contributions to $\partial_{\Delta}$ corresponding to the trees shown on Fig 17 (the parts of these graphs which are not shown are the same). They cancel each other since the corresponding trees with one 4 -valent vertex obtained by shrinking the edge $E$ inherit different orientations. Thus $\partial_{\Delta}$ is zero on the image of the map $F$.

ii) To check that the differential $\partial_{\text {Cas }}$ on $\mathcal{T}_{H, S}^{\vee, \bullet}$ match the differential $\delta_{\text {Cas }}$ for $\mathcal{C}_{H, S}^{\vee}$, look at the left picture on Fig 18. Take a cut shown by a punctured arc, and vary the trees in the two obtained domains keeping their external vertices untouched.

To check the similar statement for the map $\partial_{\mathrm{S}}$ on $\mathcal{T}_{H, S}^{\vee, \bullet}$ and the differential $\delta_{\mathrm{S}}$ on $\mathcal{C}_{H, S}^{\vee}$, look at the right picture on Fig 18, Cut out an edge $E$, cut the circle inside the arc separating the two obtained trees, and vary the trees, keeping the external vertices untouched.

Now $\partial F=F \delta$ follows from $\partial_{\mathrm{Cas}} F=F \delta_{\mathrm{Cas}}, \partial_{\mathrm{S}} F=F \delta_{\mathrm{S}}$, and $\partial_{\Delta} F=0$.

Proof of Proposition 6.1. Follows from the injectivity of the sum over plane trivalent trees map F, Lemma 6.4ii) and Theorem 6.3.

Theorem 6.5. The graded $L_{\infty}$-coalgebra $\mathcal{T}_{H, S}^{\vee, \bullet}[1]$ is a resolution of the Lie coalgebra $\mathcal{C}_{H, S}^{\vee}$. So

$$
\mathcal{C}_{H, S}^{\vee}=H_{\partial_{\Delta}}^{0}\left(\mathcal{T}_{H, S}^{\vee, \bullet}[1]\right)
$$

Proof. Follows from the exactness of (86) and Lemma 6.4 .

\section{The Hodge correlator twistor connection}

The main result of this section is Theorem 7.8 . 

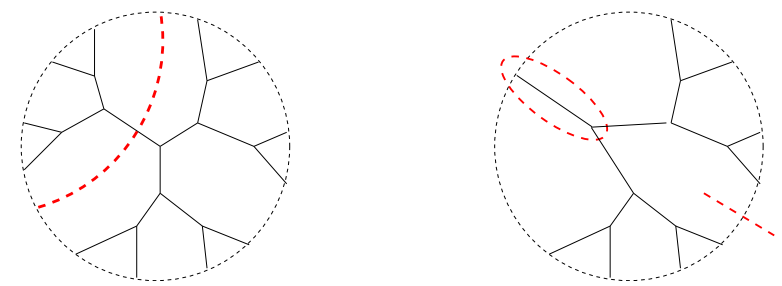

Figure 18: Compatibility of the differential $\partial_{\text {Cas }}$ (on the left) and $\partial_{\mathrm{S}}$ (on the right) on cyclic words and trees.

\subsection{Preliminary constructions}

The Hodge theoretic set-up over a base. Let $p: X \rightarrow B$ be a smooth family of complex projective curves over $B$, and $S \subset X$ a smooth divisor in $X$ over $B$. Let $s_{0}$ be a single component of $S$, and $S^{*}=S-s_{0}$. The divisor $S$ intersects each fiber at a finite collection of points, containing a special point $s_{0}$. Below we usually suppress the subscript $B$ from the notation like $X_{/ B}, S_{/ B}$. There are variations of $\mathbb{R}$-Hodge structures over $B$ :

$$
\begin{aligned}
& \mathrm{V}_{X, S^{*}}^{\vee}:=\operatorname{gr}^{W} R^{1} p_{*}(X-S, \mathbb{R})=H^{1}\left(X_{/ B}, \mathbb{R}\right) \oplus \mathbb{R}\left[S_{/ B}^{*}\right](-1), \\
& \mathrm{V}_{X, S^{*}}:=\operatorname{gr}^{W} R^{1} p_{*}(X-S, \mathbb{R})^{\vee}=H_{1}\left(X_{/ B}, \mathbb{R}\right) \oplus \mathbb{R}\left[S_{/ B}^{*}\right](1) .
\end{aligned}
$$

All objects considered in Section 6 can be upgraded to variations of $\mathbb{R}$-Hodge structures over $B$. Indeed, they are obtained by linear algebra constructions from the variation (180). In particular:

1. Consider the object

$$
\mathcal{C}_{X, S^{*}}^{\vee}(1):=\mathcal{C} \mathrm{T}\left(\mathrm{V}_{X, S^{*}}^{\vee}\right)(1)
$$

It is a Lie coalgebra in the category of variations of $\mathbb{R}$-Hodge structures over $B$. The twist by the $\mathbb{R}(1)$ is necessary to make the coproduct a morphism in this category.

2. The dual object is a Lie algebra in the same category:

$$
\mathcal{C}_{X, S^{*}}(-1):=\mathcal{C} \mathrm{T}\left(\mathrm{V}_{X, S^{*}}\right)(-1)
$$

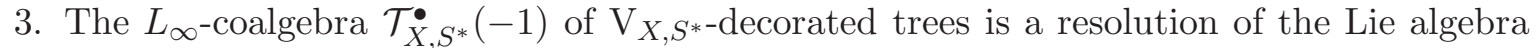
$\mathcal{C}_{X, S^{*}}(-1)$ in the same category, provided by the "sum over plane trivalent trees" map:

$$
F: \mathcal{C}_{X, S^{*}}(-1) \stackrel{\text { qis }}{\hookrightarrow} \mathcal{T}_{X, S^{*}}^{\bullet}[-1](-1) .
$$

There is a motivic set up, which works exactly the same way, starting with pure motives

$$
\mathrm{V}_{X, S^{*}}^{\vee}:=\operatorname{gr}^{W} R^{1} p_{*}(X-S), \quad \mathrm{V}_{X, S^{*}}:=\operatorname{gr}^{W} R^{1} p_{*}(X-S)^{\vee} .
$$

De Rham description of the Gauss-Manin connection. Given a smooth map $p: X \rightarrow B$, the Gauss-Manin connection $\nabla_{G M}$ in $R^{k} p_{*} \mathbb{C}$ can be described as follows. Take a section $s$ of $R^{k} p_{*} \mathbb{C}$. Choose a smooth family of differential forms $\alpha_{t}$ on the fibers $X_{t}$ whose cohomology classes form the section $s$. Choose any differential form $\beta$ on $X$ whose restriction to every fiber $X_{t}$ is the form $\alpha_{t}$. Then $d \beta$ gives rise to $\nabla_{G M}(s)$ as follows. The de Rham differential of $\beta$ along the fibers of $p$ is zero since $d \alpha_{t}=0$ for every $t$. Therefore $d \beta$ provides a 1 -form on the base with values in $\mathcal{A}_{\mathrm{cl}}^{k-1}\left(X_{/ B}\right)$. The cohomology classes of the closed $(k-1)$-forms on the fibers do not depend on the choice of the form $\beta$ representing the section $s$. The obtained 1-form on $B$ with values in $R^{k-1} p_{*} \mathbb{C}$ equals $\nabla_{G M}(s)$. 
A 1-form $\nu$. The $\mathcal{H}:=R^{1} p_{*}(\mathbb{R})^{\vee}$ is a local system on $B$ with the Gauss-Manin connection $\nabla_{G M}$ and a fiber $H_{1}\left(X_{t}, \mathbb{R}\right)$ over $t \in B$. By definition, $H_{1}\left(X_{t}, \mathbb{Z}\right)$ is flat for the connection.

The Albanese variety $A_{X_{t}}$ of $X_{t}$ is identified as a manifold with $H_{1}\left(X_{t}, \mathbb{R}\right) / H_{1}\left(X_{t}, \mathbb{Z}\right)$. The fibers of the local system $\mathcal{H}$ are the tangent spaces to Albanese varieties $A_{X_{t}}$.

Let $\pi: X \times{ }_{B} X \rightarrow B$ be the fibered product of $X$ over $B$. Then there is a $C^{\infty} 1$-form

$$
\nu \in \mathcal{A}_{X \times_{B} X}^{1} \otimes \pi^{*} \mathcal{H}
$$

on $X \times_{B} X$ with values in the local system $\pi^{*} \mathcal{H}$. Its value at a tangent vector $\left(v_{1}, v_{2}\right)$ at $\left(x_{1}, x_{2}\right)$ is obtained as follows. Let $\left(x_{1}(t), x_{2}(t)\right)$ be a smooth path from $\left(x_{1}, x_{2}\right)$ in the direction $\left(v_{1}, v_{2}\right)$. Assuming that the points $\left(x_{1}(t), x_{2}(t)\right)$ are in the same fiber $X_{t}$, we get a section $\left(x_{1}(t)-x_{2}(t)\right) \in A_{X_{t}}$. The Gauss-Manin connection provides another section of the Albanese fibration passing through $\left(x_{1}(0)-x_{2}(0)\right)$. We define $\nu\left(v_{1}, v_{2}\right)$ to be the difference of the tangents to these sections with the velocity $\left(v_{1}, v_{2}\right)$.

Green functions for a family of curves. Let $\mathcal{H}_{\mathbb{C}}:=\mathcal{H} \otimes \mathbb{C}=\mathcal{H}_{\mathbb{C}}^{-1,0} \oplus \mathcal{H}_{\mathbb{C}}^{0,-1}$ be the Hodge decomposition. The intersection pairing $H_{1}\left(X_{t}, \mathbb{Z}\right) \wedge H_{1}\left(X_{t}, \mathbb{Z}\right) \longrightarrow \mathbb{Z}(1)$ provides a pairing

$$
\langle *, *\rangle: \mathcal{H}_{\mathbb{C}}^{-1,0} \otimes \mathcal{H}_{\mathbb{C}}^{0,-1} \longrightarrow \mathbb{C} .
$$

The wedge product $\nu \wedge \nu$, followed by the pairing $\langle *, *\rangle$, provides a canonical $(1,1)$-form

$$
\langle\nu \wedge \nu\rangle \in \mathcal{A}_{X \times_{B} X}^{1,1} \cdot
$$

Denote by $\langle\nu \wedge \nu\rangle^{[1 \times 1]}$ its $1 \times 1$-component on $X \times_{B} X$. Let $\delta_{\Delta}$ be the $\delta$-function of the relative diagonal $\Delta \subset X \times_{B} X$. A section $a: B \rightarrow X$ provides a divisor $S_{a} \subset X \times_{B} X$, whose fiber at $t \in \mathcal{B}$ is $a_{t} \times X_{t} \cup X_{t} \times a_{t}$. It gives rise to a $(1,1)$-current $\delta_{S_{a}}$ on $X$.

Definition 7.1. Let $p: X \rightarrow B$ be a proper map and $a: B \rightarrow X$ is its section. A Green current $G_{a}(x, y)$ is a 0 -current on $X \times_{B} X$ satisfying the equation

$$
\bar{\partial} \partial G_{a}(x, y)=\delta_{\Delta}-\delta_{S_{a}}-\langle\nu \wedge \nu\rangle^{[1 \times 1]} .
$$

The Green function exists fiberwise by the $\bar{\partial} \partial$-lemma. It determines a 0 -current $G_{a}(x, y)$ uniquely up to a function lifted from the base $B(\mathbb{C})$. Let us choose a non vanishing $v \in T_{a} X$. It provides a uniquely defined normalized Green function $G_{v}(x, y)$.

Below we choose a section $v_{0}$ of the fibration $T_{s_{0}} X_{/ B}$, providing us a Green function $G_{v_{0}}(x, y)$.

\subsection{The Hodge correlator twistor connection}

Let us define the Hodge correlator map in a bit more general set-up.

Let $T$ be a plane tree, not necessarily trivalent. We assume that a tree does not have two-valent vertices. We proceed similarly to Section 2.3. We start with a decorated tree $(T, W)$.

i) Suppose that the $T$ has more then one edge. Euivalently, it has an internal vertex. Consider the fibered product over the base $B$ of the copies of $X$ parametrised by the vertices of $T$.

$$
\mathcal{X}_{T}:=X_{/ B}^{\{\text {vertices of } T\}} .
$$


Let $\left\{E_{0}, \ldots, E_{r}, F_{1}, \ldots, F_{k}\right\}$ be the edges of $T$ numbered so that the edges $E_{0}, \ldots, E_{r}$ are internal or $S^{*}$-decorated, and the edges $F_{1}, \ldots, F_{k}$ are decorated by sections $\omega_{F_{1}}, \ldots, \omega_{F_{k}}$ of the local system $\operatorname{gr}_{W} H^{1}(X-S, \mathbb{R})$. We assume that the order $F_{1}, \ldots, F_{k}$ is compatible with the cyclic order provided by the orientation of the plane containing the tree $T$. Choose a 1-form $\alpha_{F_{j}}$ on $X$ representing a section $\omega_{F_{j}}$ : its restriction to a fiber $X_{t}$ is a holomorphic/antiholomorphic form.

Consider an element of the orientation torsor of the tree $T$ :

$$
\left(E_{0} \wedge \ldots \wedge E_{r}\right) \wedge\left(F_{1} \wedge \ldots \wedge F_{k}\right) \in \text { or }_{T}
$$

The difference between the element (186) and a given orientation $\mathrm{Or}_{T} \in \mathrm{or}_{T}$ is denoted by

$$
\operatorname{sgn}_{\mathrm{Or}_{T}}\left(E_{0} \wedge \ldots \wedge E_{r} \wedge F_{1} \wedge \ldots \wedge F_{k}\right):=(186) / \mathrm{Or}_{T} \in\{ \pm 1\} .
$$

We assign to each edge $E_{i}$ the Green current $G_{E_{i}}$ on $X \times_{B} X$, where the copies of $X$ match the vertices of the edge $E_{i}$. Since the Green current is symmetric, it does not depend on the choice of an orientation of the edge $E_{i}$.

Recall the twistor plane $\mathbb{C}^{2}$ with coordinates $(z, w)$. Using the forms $\alpha_{F_{j}}$, the Green functions $G_{E_{i}}$ at the edges of $T$, and the orientation $\mathrm{Or}_{T}$, let us cook up a current $\widehat{\kappa}\left(T, W ; \mathrm{Or}_{T}\right)$ on

$$
\mathcal{X}_{T} \times \mathbb{C}^{2} .
$$

Put the form $\alpha_{F_{j}}$ to the copy of $X / B$ assigned to the unique internal vertex of the edge $F_{j}$. Abusing notation, we identify it with its pull back to $\mathcal{X}_{T} \times \mathbb{C}^{2}$. Recall

$$
\mathrm{D}^{\mathbb{C}}:=(w \partial-z \bar{\partial})+(z d w-w d z) .
$$

Definition 7.2. We define a differential form on (187) by setting

$$
\begin{gathered}
\widehat{\kappa}\left(T, W ; \mathrm{Or}_{T}\right):= \\
\operatorname{sgn}_{\mathrm{Or}_{T}}\left(E_{0} \wedge \ldots \wedge E_{r} \wedge F_{1} \wedge \ldots \wedge F_{k}\right) \mathrm{D}^{\mathbb{C}} G_{E_{0}} \wedge \ldots \wedge \mathrm{D}^{\mathbb{C}} G_{E_{r}} \wedge \alpha_{F_{1}} \wedge \ldots \wedge \alpha_{F_{k}} .
\end{gathered}
$$

Theorem 7.3. The form $\widehat{\kappa}\left(T, W ; \mathrm{Or}_{T}\right)$ a current on $\mathcal{X}_{T} \times \mathbb{C}^{2}$, algebraic along the twistor plane:

$$
\widehat{\kappa}\left(T, W ; \mathrm{Or}_{T}\right) \in \mathcal{D}^{*, *}\left(\mathcal{X}_{T}\right) \otimes_{\mathbb{C}} \Omega_{\mathbb{C}^{2}}^{\leq 1}
$$

Proof. Just as in Lemma 2.2.

There is a canonical projection

$$
p_{T}: \mathcal{X}_{T}^{\text {int }} \times \mathbb{C}^{2}:=X_{/ B}^{\{\text {internal vertices of } T\}} \times \mathbb{C}^{2} \longrightarrow B \times \mathbb{C}^{2} .
$$

Definition 7.4. Suppressing $v_{0}$ from the notation, set

$$
\operatorname{Cor}_{\mathcal{H}}\left(T ; \mathrm{Or}_{T}\right)(W):=p_{T *}\left(\widehat{\kappa}\left(T, W ; \mathrm{Or}_{T}\right)\right) \in \mathcal{D}^{*, *}(B) \otimes_{\mathbb{C}} \Omega_{\mathbb{C}^{2}}^{\leq 1}
$$



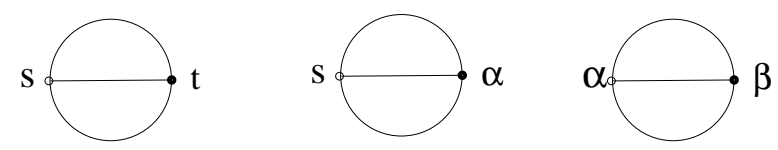

Figure 19: The three possible decorations of a single edge tree.

When $B$ is a point, we recover the Hodge correlator map from Definition 2.6. In this case the form (189) does not depend on the choice of forms $\alpha_{F_{j}}$. Indeed, if $\widetilde{\alpha}_{F_{j}}$ is a different choice, then the restriction of $\widetilde{\alpha}_{F_{j}}-\alpha_{F_{j}}$ to the fiber curve is zero. Indeed, the fibers are smooth complex projective curves, and so there are canonical harmonic representatives for $H^{1}$ given by holomorphic/antiholomorphic forms. The same is true for the components of the Hodge correlator which are functions on the base. In particular, this is the case in the crucial for us case when $T$ is a plane trivalent tree, as we will see in Proposition 7.7 .

ii) Let $T$ be a tree with a single edge $E$. Since there are no internal vertices, the definition of the Hodge correlator involves no integration. There are three possible decorations, see Fig 19.

1. $T=T_{1}$ is decorated by sections $s, t \in S^{*}$. The sections provide a map $(s, t): B \rightarrow X \times{ }_{B} X$. We assign to $T_{1}$ the pull back of the Green current $G(s, t):=(s, t)^{*} G$ to the base $B$ :

$$
\operatorname{Cor}_{\mathcal{H}}\left(T_{1}\right)(\{s\} \otimes\{t\}):=G(s, t) \in \mathcal{D}_{B}^{0} \otimes \mathbb{R}(-1) .
$$

Dualising, and since the dual to $\{s\} \otimes\{t\}$ is a section of the variation $\mathbb{R}(2)$ on $B$, we get

$$
\operatorname{Cor}_{\mathcal{H}}\left(T_{1}\right):=G(s, t) \otimes(\{s\} \otimes\{t\})^{\vee} \in \mathcal{D}_{B}^{0} \otimes \mathbb{R}(1) \subset \mathcal{C}_{\mathcal{H}_{\mathbb{R}}}^{1}\left(\mathcal{C}_{X, S^{*}}\right) .
$$

To check the last inclusion, notice that $\mathbb{R}(1)$-valued 0 -forms have degree 1 in the Hodge complex according to (94), and that the Green function $G(s, t)$ is imaginary, in agreement with the reality condition (93) on the Hodge complex. The Hodge bidegree is $(-1,-1)$.

2. $T=T_{2}$ is a single edge, decorated by a section $s: B \rightarrow X$ and a 1 -form $\alpha$ on $X$. We set

$$
\operatorname{Cor}_{\mathcal{H}}\left(T_{2}\right)(\{s\} \otimes \alpha):=s^{*} \alpha \in \mathcal{A}_{B}^{1} .
$$

Let us dualise this element. Let $s^{*} \alpha \mid h$ be the canonical Casimir element, defined as follows. Choose a basis $\alpha_{1}, \ldots, \alpha_{2 g}$ in $\mathcal{H}^{\vee}=R^{1} p_{*}(\mathbb{R})$, the dual basis $h_{1}, \ldots, h_{2 g}$ in $\mathcal{H}=R^{1} p_{*}(\mathbb{R})^{\vee}$, and set

$$
s^{*} \alpha \mid h:=\sum_{i=1}^{2 g} s^{*} \alpha_{i} \otimes h_{i} \in \mathcal{H}^{\vee} \otimes \mathcal{H} .
$$

Dualising map (192), using the fact that the dual to $\{s\} \otimes \alpha$ is a section of the variation of real Hodge structures $\mathcal{H}(1)$ on $B$, and twisting by $\mathbb{R}(-1)$, we get an element

$$
\operatorname{Cor}_{\mathcal{H}}\left(T_{2}\right):=s^{*} \alpha \mid h \otimes \mathbb{R}(-1) \in \mathcal{A}_{B}^{1,0} \otimes \mathcal{H}^{-1,0} \oplus \mathcal{A}_{B}^{0,1} \otimes \mathcal{H}^{0,-1} \subset \mathcal{C}_{\mathcal{H}_{\mathbb{R}}}^{1}\left(\mathcal{C}_{X, S^{*}}\right)
$$

Each summand has degree 1 in the Hodge complex, see (94). The Hodge bidegree is $(0,0)$. It is imaginary since $s^{*} \alpha \mid h$ is real, in agreement with reality condition (93) on the Hodge complex.

3. $T$ is a single edge decorated by forms $\alpha, \beta$. We assign to it zero. 
The Hodge correlator class. Let $W=\left(x_{1} \otimes \ldots \otimes x_{m}\right)_{\mathcal{C}}$. Let $|\operatorname{Aut}(W)|$ be the order of its automorphism group. Let $\mathcal{C}$ be the operator of weighted projection on the cyclic tensor algebra:

$$
\mathcal{C}\left(x_{1} \otimes \ldots \otimes x_{m}\right):=\frac{1}{|\operatorname{Aut}(W)|}\left(x_{1} \otimes \ldots \otimes x_{m}\right)_{\mathcal{C}}
$$

Given a collection of cohomology classes $\gamma_{i} \in V_{X, S^{*}}^{\vee}$ and homology classes $h_{i} \in V_{X, S^{*}}$, we define the Hodge correlator map of the cyclic tensor product of the expressions $\gamma_{i} \mid h_{i}$ as a cyclic product of their homology factors taken with the coefficient given by the Hodge correlator map applied to the cyclic product of the corresponding differential form factors.

Here is an example. Let $\alpha_{1}, \alpha_{2}, \alpha_{3}$ be forms on $X$. Then

$$
\begin{gathered}
\operatorname{Cor}_{\mathcal{H}}\left(\alpha_{1}\left|h_{1} \otimes \alpha_{2}\right| h_{2} \otimes \alpha_{3} \mid h_{3}\right):= \\
\operatorname{Cor}_{\mathcal{H}}\left(\alpha_{1} \otimes \alpha_{2} \otimes \alpha_{3}\right) \cdot\left(h_{1} \otimes h_{2} \otimes h_{3}\right)_{\mathcal{C}}=\int_{X / B}\left(\alpha_{1} \wedge \alpha_{2} \wedge \alpha_{3}\right) \cdot\left(h_{1} \otimes h_{2} \otimes h_{3}\right)_{\mathcal{C}} .
\end{gathered}
$$

Choose a basis $\left\{\gamma_{k}\right\}$ in $V_{X, S^{*}}^{\vee}$. Let $h_{\gamma_{k}}$ be the dual basis in $V_{X, S^{*}}$. Set

$$
\gamma\left|h:=\sum_{k} \gamma_{k}\right| h_{\gamma_{k}}
$$

Take a plane oriented tree $\left(T, \mathrm{Or}_{T}\right)$, not necessarily trivalent, with $m$ external vertices. Decorate the tree $T$ by the cyclic word $\mathcal{C}(\gamma|h \otimes \ldots \otimes \gamma| h)$, the $m$-fold cyclic tensor product of the $\gamma \mid h$ 's. By this we mean decorating the $T$ by the $m$-fold tensor product $\gamma|h \otimes \ldots \otimes \gamma| h$ and all its cyclic shifts. We apply the Hodge correlator map to this tree, decorated by the $\mathcal{C}(\gamma|h \otimes \ldots \otimes \gamma| h)$ :

$$
\operatorname{Cor}_{\mathcal{H}}\left(T, \mathrm{Or}_{T}\right) \mathcal{C}(\gamma|h \otimes \ldots \otimes \gamma| h) .
$$

A plane trivalent tree $T$ has a canonical orientation $\mathrm{Or}_{T}$, induced by the plane orientation.

Definition 7.5. The Hodge correlator class $\mathbf{G}$ is obtained by taking the sum of Hodge correlators 197) over all plane trivalent trees $T$, with the canonical orientation $\mathrm{Or}_{T}$, twisted by $\mathbb{R}(-1)$ :

$$
\mathbf{G}:=\sum_{m \geq 2} \sum_{T} \sum_{W} \frac{1}{|\operatorname{Aut}(W)|} \operatorname{Cor}_{\mathcal{H}}\left(T, \mathrm{Or}_{T}\right)\left(\gamma_{1}\left|h_{\gamma_{1}} \otimes \ldots \otimes \gamma_{m}\right| h_{\gamma_{m}}\right)(-1) .
$$

Here $\{W\}=\left\{\gamma_{1} \otimes \ldots \otimes \gamma_{m}\right\}$ is a basis in $\mathcal{C}\left(\mathrm{T}\left(V_{X, S^{*}}^{\vee}\right)\right)$.

One can rephraise Definition 7.5 as follows. Set

$$
\mathrm{I}:=1+\gamma\left|h_{\gamma}+\gamma\right| h \otimes \gamma|h+\gamma| h \otimes \gamma|h \otimes \gamma| h+\ldots
$$

Applying the operator $\mathcal{C}$, see (194), we get a cyclic Casimir element:

$$
\mathcal{C}(\mathrm{I}):=\mathcal{C}(1+\gamma|h+\gamma| h \otimes \gamma|h+\gamma| h \otimes \gamma|h \otimes \gamma| h+\ldots) .
$$

Definition 7.6. The Hodge correlator class is obtained by applying to (200) the Hodge correlator map, twisted by $\mathbb{R}(-1)$, and taking the sum over all plane trivalent trees $T$ :

$$
\mathbf{G}:=\sum_{T} \operatorname{Cor}_{\mathcal{H}}\left(T, \mathrm{Or}_{T}\right)(\mathcal{C}(\mathrm{I})(-1))
$$


Explicitly, the Hodge correlator class is given by:

$$
\begin{aligned}
& \mathbf{G}=\mathbf{G}_{0,0}+\sum_{s, t \geq 0} z^{s} w^{t}\left((z d w-w d z) \wedge(s+t+1) \mathbf{G}_{s+1, t+1}+\left(w \partial^{\prime}-z \partial^{\prime \prime}\right) \mathbf{G}_{s+1, t+1}\right), \\
& \mathbf{G}_{0,0}:=\operatorname{Cor}_{\mathcal{H}}\left(s^{*} \alpha \mid h_{\alpha}\right)+\operatorname{Cor}_{\mathcal{H}}\left(\alpha_{1}\left|h_{\alpha_{1}} \otimes \alpha_{2}\right| h_{\alpha_{2}} \otimes \alpha_{3} \mid h_{\alpha_{3}}\right)_{\mathcal{C}} .
\end{aligned}
$$

For example, $\mathbf{G}_{1,1}=G(a, b)$ is the Green function.

Recall the differential $d_{\mathbb{C}^{2}}$ on the twistor plane $\mathbb{C}^{2}$. Recall the projection

$$
\pi: B \times \mathbb{C}^{2} \rightarrow B
$$

The Hodge correlator class $\mathbf{G}$ is a current on $B \times \mathbb{C}^{2}$ with values in the local system of Lie algebras $\pi^{*} \mathcal{C}_{X, S^{*}}(-1)$ with a flat connection $\mathbf{d}+\mathrm{d}_{\mathbb{C}^{2}}$. This local system is a Lie algebra in the category of variations of $\mathbb{R}$-Hodge structures on $B \times \mathbb{C}^{2}$.

Recall the Lie algebra $\pi^{*} \mathcal{C} \mathcal{L} i e_{X, S^{*}}(-1)$, see (24). It is obtained by linear algebra constructions (21), (22) from variations of $\mathbb{R}$-Hodge structures on $B \times \mathbb{C}^{2}$. So it is a Lie subalgebra of $\pi^{*} \mathcal{C}_{X, S^{*}}(-1)$ in the same category. Its underlying flat connection is $\mathbf{d}+\mathrm{d}_{\mathbb{C}^{2}}$.

Lemma 7.7. The Hodge correlator $\mathbf{G}$ is a $\pi^{*}\left(\mathcal{C} \mathcal{L} i e_{X, S^{*}}\right)(-1)$-valued 1 -form on $B \times \mathbb{C}^{2}$.

Proof. The Hodge correlator $\mathbf{G}$ satisfies the shuffle relations, see Section 3.3, This just means that $\mathbf{G}$ is a $\pi^{*}\left(\mathcal{C} \mathcal{L}_{i} e_{X, S^{*}}\right)(-1)$-valued current on $B \times \mathbb{C}^{2}$. A plane trivalent tree with $n$ vertices has $2 n+1$ edges. In the integrand (188) we assign a 1 -form to each edge. Since $(2 n+1)-2 n=1$, after the integration we get a 1 -form on $B \times \mathbb{C}^{2}$.

The Hodge correlator twistor connection $\nabla_{\mathbf{G}}$. Lemma 7.7 implies that the Hodge correlator $\mathbf{G}$ gives rise to a connection on $B \times \mathbb{C}^{2}$ with values in the Lie algebra $\pi^{*}\left(\mathcal{C} \mathcal{L} i e_{X, S^{*}}(-1)\right)$ in the category of variations of real Hodge structures:

$$
\nabla_{\mathbf{G}}:=\mathbf{d}+\mathrm{d}_{\mathbb{C}^{2}}+\mathbf{G} .
$$

We call it the Hodge correlator twistor connection $\nabla_{\mathbf{G}}$. The name is justified by Theorem 7.8 .

Theorem 7.8. The connection $\nabla_{\mathbf{G}}$ is a twistor connection on $B \times \mathbb{C}^{2}$. This just means that:

- The connection $\nabla_{\mathbf{G}}$ is $\mathbb{C}^{*} \times \mathbb{C}^{*}$-equivariant real connection on $B \times \mathbb{C}^{2}$.

- The restriction of the connection $\nabla_{\mathbf{G}}$ to $B \times \mathbb{C}$ is flat. Equivalently, the restriction $\mathbf{G}^{\prime}$ of the Hodge correlator $\mathbf{G}$ to the twistor line $z+w=1$ satisfies the Maurer-Cartan equation

$$
\left(\mathbf{d}+\mathrm{d}_{\mathbb{C}}\right) \mathbf{G}^{\prime}+\mathbf{G}^{\prime} \wedge \mathbf{G}^{\prime}=0 .
$$

The Hodge correlator class $\mathbf{G}$ in (202) is nothing else but the twistor transform of the data

$$
\mathbf{G}^{*}:=\left(\mathbf{G}_{0,0},\left\{\mathbf{G}_{s, t}\right\}_{s, t \geq 1}\right) .
$$

Theorem 7.8 just means that $\mathbf{G}^{*}$ is a Green data. Since $\pi^{*}\left(\mathcal{C} \mathcal{L} i e_{X, S^{*}}(-1)\right)$ is a Lie algebra in the category of variations of $\mathbb{R}$-Hodge structures, its Hodge complex $\mathcal{C}_{\mathcal{H}_{\mathbb{R}}}\left(\mathcal{C} \mathcal{L} i e_{X, S^{*}}(-1)\right)$ is defined, and it has a DG Lie algebra structure, see Section 4.1. So we can restate Theorem 7.8 as follows: 
Theorem 7.9. The data $\mathbf{G}^{*}$ defines a 1-cocycle in the Hodge complex:

$$
\mathbf{G}^{*} \in \mathcal{C}_{\mathcal{H}}^{1}\left(\mathcal{C} \mathcal{L} i e_{X, S^{*}}(-1)\right) .
$$

One of the benefits of Definition 7.2 is that it delivers the twistor connection $\nabla_{\mathbf{G}}$ directly. So the twistor transform is built naturally in the construction of Hodge correlators.

The construction from Section 2 delivers the Green data $\mathbf{G}^{*}$, and we need to apply to it the twistor transform, which seems to come out of the blue, to get the twistor connection $\nabla_{\mathbf{G}}$.
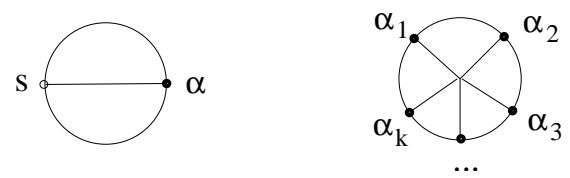

Figure 20: The decorated trees providing Hodge correlators of the Hodge bidegree $(0,0)$.

Remark. Denote by $e_{T, W}$ the number of edges of a plane decorated tree $(T, W)$, not necessarily trivalent, which are not external edges with a 1-form in the decoration. It is the number of the edges of $T$ to which we assign Green currents. Then $e_{T, W}$ is the $\mathrm{N}$-degree of the Hodge correlator $\operatorname{Cor}_{\mathcal{H}}(T, W)$. Indeed, the $e_{T, W}$ is the total degree of the $\operatorname{Cor}_{\mathcal{H}}(T, W)$ as a polynomial in $(z, w)$, e.g. the degree of $z d w-w d z$ is 1 , which coinsides with the N-degree of the Hodge correlator $\operatorname{Cor}_{\mathcal{H}}(T, W)$. In particular, the $\mathbf{G}_{0,0}$ is the contribution of the decorated plane trivalent trees with $e_{T, W}=0$. Here are couple of examples.

If $e_{T, W}=0$, then $(T, W)$ is one of the two decorated trees on Fig 20.

If $e_{T, W}=1$, then $(T, W)$ is one of the three decorated trees on Fig 21 .
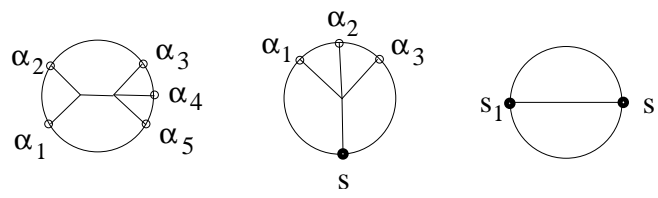

Figure 21: The decorated trees providing Hodge correlators of the Hodge bidegree $(-1,-1)$.

\subsection{Proof of Theorem 7.8}

We prove Theorem 7.8 in three steps.

1) Establish Proposition [7.7. Show that the connection $\nabla_{\mathbf{G}}$ is $\mathbb{C}^{*} \times \mathbb{C}^{*}$-equivariant and real.

2) Show that the Hodge correlator $\mathrm{Cor}_{\mathcal{H}}$ is multiplicative.

3) Prove the Maurer-Cartan formula (204) for the differential of the Hodge correlator $\mathbf{G}^{\prime}$.

1) The $\nabla_{\mathbf{G}}$ is a $\mathbb{C}^{*} \times \mathbb{C}^{*}$-equivariant real connection. First, let us consider the first component of $\mathbf{G}_{0,0}$. It is the restriction of the real Casimir element $\gamma \mid h$ to a section $s$, see (193). So it is real, and its Hodge bidegree is $(0,0)$, see (193). The rest is done as follows.

The Casimir element $\gamma \mid h$ is of the Hodge bidegree $(0,0)$. The form $\mathrm{D}^{\mathbb{C}} G$ is of the Hodge bidegree $(1,1)$. For any plane trivalent tree $T$ the number of the edges $E$ on which we put the form $\mathrm{D}^{\mathbb{C}} G$ is one less then the number of the internal vertices. Indeed, this is true if all external 
edges are decorated by 1-forms; adding an $s$-decorated external edge amounts to adding one internal edge, and one internal vertex. Since integration over the relative curve $X / B$ has the Hodge bidegree $(-1,-1)$, and since we twisted the result by $\mathbb{R}(-1)$, the total bidegree is $(0,0)$. This just means that the connection $\nabla_{\mathbf{G}}$ is $\mathbb{C}^{*} \times \mathbb{C}^{*}$-equivariant.

Recall the antiholomorphic involution of the twistor plane $\sigma:(z, w) \longrightarrow(\bar{w}, \bar{z})$, and the complex conjugatoon $c$. For the involution $c \circ \sigma$, the both the operator $\mathrm{D}^{\mathbb{C}}$ and the Green current are imaginary, and therefore $\mathrm{D}^{\mathbb{C}} G$ is real:

$$
(c \circ \sigma)^{*} \mathrm{D}^{\mathbb{C}}=-\mathrm{D}^{\mathbb{C}}, \quad(c \circ \sigma)^{*} G=-G, \quad(c \circ \sigma)^{*} \mathrm{D}^{\mathbb{C}} G=\mathrm{D}^{\mathbb{C}} G .
$$

The Casimir element $\gamma \mid h$ is evidently real. Therefore the integrand (188) is real. This implies that the Hodge correlator class $\mathbf{G}$ is real. We conclude that the connection $\nabla_{\mathbf{G}}$ is real.

So if $s, t \geq 1$, the $\mathbf{G}_{s, t}$ is a $\mathcal{C} \mathcal{L} i e_{X, S^{*}}(-1)$-valued function on $B$ of the Hodge bidegree $(-s,-t)$. The $\mathbf{G}_{0,0}$ is a $\mathcal{C} \mathcal{L} i e_{X, S^{*}}(-1)$-valued 1 -form on $B$.

2. Multiplicativity of the Hodge correlators. The Hodge correlator construction can be applied to an oriented forest $\left(F ; \mathrm{Or}_{F}\right)$, resulting an element in the Hodge complex:

$$
\operatorname{Cor}_{\mathcal{H}}\left(F ; \operatorname{Or}_{F}\right) \in \mathcal{C}_{\mathcal{H}}^{\bullet}\left(S^{\bullet}\left(\mathcal{C}_{X, S^{*}}(-1)\right)\right) \text {. }
$$

Since $S^{\bullet}\left(\mathcal{C}_{X, S^{*}}(-1)\right)$ is a graded commutative algebra in the category of $\mathbb{R}$-Hodge structures, its Hodge complex $\mathcal{C}_{\mathcal{H}_{\mathbb{R}}}^{\bullet}\left(S^{\bullet}\left(\mathcal{C}_{X, S^{*}}(-1)\right)\right)$ is a graded commutative algebra, with the product $*$.

Proposition 7.10. The Hodge correlator map (206) is multiplicative:

$$
\operatorname{Cor}_{\mathcal{H}}\left(\left(F^{\prime} ; \operatorname{Or}_{F^{\prime}}\right) \circ\left(F^{\prime \prime} ; \operatorname{Or}_{F^{\prime \prime}}\right)\right)=\operatorname{Cor}_{\mathcal{H}}\left(F^{\prime} ; \operatorname{Or}_{F^{\prime}}\right) * \operatorname{Cor}_{\mathcal{H}}\left(F^{\prime \prime} ; \operatorname{Or}_{F^{\prime \prime}}\right) \text {. }
$$

Proof. One has $\mathcal{X}_{F^{\prime} \times F^{\prime \prime}}=\mathcal{X}_{F^{\prime}} \times \mathcal{X}_{F^{\prime \prime}}$. There is a canonical direct product map of distributions

$$
\mathcal{D}^{*, *}\left(\mathcal{X}_{F^{\prime}}\right) \times \mathcal{D}^{*, *}\left(\mathcal{X}_{F^{\prime \prime}}\right) \longrightarrow \mathcal{D}^{*, *}\left(\mathcal{X}_{F^{\prime} \times F^{\prime \prime}}\right) \text {. }
$$

The current $\widehat{\kappa}\left(F, W ; \operatorname{Or}_{F}\right)$ assigned to a decorated forest $F$, see (188), is evidently multiplicative:

$$
\widehat{\kappa}\left(F^{\prime}, W^{\prime} ; \mathrm{Or}_{F^{\prime}}\right) \times \widehat{\kappa}\left(F^{\prime \prime}, W^{\prime \prime} ; \mathrm{Or}_{F^{\prime \prime}}\right)=\widehat{\kappa}\left(F^{\prime} \times F^{\prime \prime}, W^{\prime} \times W^{\prime \prime} ; \mathrm{Or}_{F^{\prime} \times F^{\prime \prime}}\right) .
$$

Recall the projection $p_{F}: \mathcal{X}_{F}^{\text {int }} \times \mathbb{C}^{2} \longrightarrow B \times \mathbb{C}^{2}$. Provided that the $S^{*}$-decorations of $F^{\prime}$ and $F^{\prime \prime}$ are disjoint, the multiplicativity implies that

$$
p_{F^{\prime}}\left(\widehat{\kappa}\left(F^{\prime}, W^{\prime} ; \operatorname{Or}_{F^{\prime}}\right)\right) \wedge p_{F^{\prime \prime}}\left(\widehat{\kappa}\left(F^{\prime \prime}, W^{\prime \prime} ; \operatorname{Or}_{F^{\prime \prime}}\right)\right)=p_{F^{\prime} \times F^{\prime \prime}}\left(\widehat{\kappa}\left(F^{\prime} \times F^{\prime \prime}, W^{\prime} \times W^{\prime \prime} ; \operatorname{Or}_{F^{\prime} \times F^{\prime \prime}}\right)\right) .
$$

Since the Hodge complex functor $\mathcal{C}_{\mathcal{H}}$ is multiplicative, this implies the claim.

3. Maurer-Cartan differential equations for the Hodge correlators. Recall that the Hodge correlator $\operatorname{Cor}_{\mathcal{H}}\left(T ; \mathrm{Or}_{T}\right)$ is the push forward to $B \times \mathbb{C}^{2}$ of the current

$$
\mathrm{D}^{\mathbb{C}} G_{E_{0}} \wedge \ldots \wedge \mathrm{D}^{\mathbb{C}} G_{E_{r}} \wedge \gamma|h \wedge \ldots \wedge \gamma| h .
$$


Theorem 7.11. The following diagram is commutative:

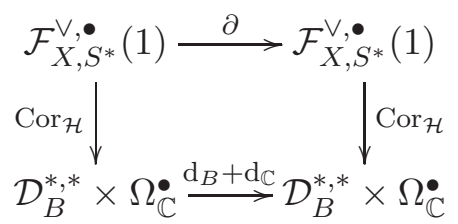

This means that for any decorated oriented forest $\left(F, W ; \mathrm{Or}_{F}\right)$ we have

$$
\left(\mathrm{d}_{B}+\mathrm{d}_{\mathbb{C}}\right) \operatorname{Cor}_{\mathcal{H}}\left(F, W ; \operatorname{Or}_{F}\right)=\operatorname{Cor}_{\mathcal{H}} \circ \partial\left(F, W ; \operatorname{Or}_{F}\right) .
$$

Proof. It is sufficient to prove the result for an oriented decorated tree $\left(T, \mathrm{Or}_{T}\right)$. To calculate the differential $\mathrm{d}_{B}+\mathrm{d}_{\mathbb{C}}$ on $B \times \mathbb{C}^{2}$, we apply the de Rham differential $\mathrm{d}+\mathrm{d}_{\mathbb{C}}$ to the integrand, and use the fact that the de Rham differential commutes with the proper push forward. The Casimir element $\gamma \mid h$ represents the identity map, and thus is annihilated by the differential. Therefore the calculation of the value of $\left(\mathrm{d}+\mathrm{d}_{\mathbb{C}}\right) \operatorname{Cor}_{\mathcal{H}}\left(T, W ; \mathrm{Or}_{T}\right)$ boils down to the calculation of the differential

$$
\left(\mathrm{d}+\mathrm{d}_{\mathbb{C}}\right) \mathrm{D}^{\mathbb{C}} G_{E_{0}} \wedge \ldots \wedge \mathrm{D}^{\mathbb{C}} G_{E_{r}} .
$$

Recall the Laplacian $\Delta=\partial^{\prime \prime} \partial^{\prime}$. We employ formula (65) to calculate (209). Namely:

$$
\left(\mathrm{d}+\mathrm{d}_{\mathbb{C}}\right) \mathrm{D}^{\mathbb{C}} G_{E_{0}} \wedge \ldots \wedge \mathrm{D}^{\mathbb{C}} G_{E_{r}}=\Delta G_{E_{0}} \wedge \mathrm{D}^{\mathbb{C}} G_{E_{1}} \ldots \wedge \mathrm{D}^{\mathbb{C}} G_{E_{r}}+\ldots
$$

We use formula (74) for the Laplacian:

$$
\Delta G_{s_{0}}(x, y)=\delta_{\Delta_{X}}-\left(p_{1}^{*} \delta_{s_{0}}+p_{2}^{*} \delta_{s_{0}}-\frac{i}{2} \sum_{k=1}^{g}\left(p_{1}^{*} \alpha_{k} \wedge p_{2}^{*} \bar{\alpha}_{k}+p_{2}^{*} \alpha_{k} \wedge p_{1}^{*} \bar{\alpha}_{k}\right)\right) .
$$

There are three terms in the formula for the Laplacian:

(a) The $\delta_{\Delta_{X}}$-term.

(b) The volume term $p_{1}^{*} \delta_{s_{0}}+p_{2}^{*} \delta_{s_{0}}$.

(c) The reduced Casimir term - the remaining term.

We call the sum of the terms (b)+(c) the Casimir term.

Let us calculate the contribution of the Laplacian corresponding to an edge $E$. We will show that each term of the contribution matches certain term in the form $\operatorname{Cor}_{\mathcal{H}} \circ \partial$.

1. $E$ is an internal edge. Then there are three cases, matching the terms in $\Delta G$.

(1a) The $\delta_{\Delta_{X}}$-term contributes the diagram with a special vertex, obtained by shrinking the edge $E$, see the right of Fig [1] This matches the graph complex differential $\partial_{\Delta}$.

(1b) Let us show that the contribution of the volume term is zero. Let $T_{1}$ and $T_{2}$ be the two trees obtained by cutting $T$ along the edge $E$. We assign the current $\delta_{s_{0}}$ to the end of one of them, say $T_{1}$. Denote by $W_{1}$ the induced decoration of $T_{1}$. Let us show that $\operatorname{Cor}_{\mathcal{H}, v_{0}}\left(T_{1}, W_{1} ; \mathrm{Or}_{T_{1}}\right)=0$.

Recall the regularisation procedure called the specialization at a tangent vector, Section 3.1 . One defines specializations of forms by taking specializations of their coefficients.

Lemma 7.12. Let $W=\mathcal{C}\left(\{s\} \otimes W_{0}\right)$. Then the specialization $\operatorname{Sp}_{v_{0}}^{s \rightarrow s_{0}} \operatorname{Cor}_{\mathcal{H}, v_{0}}(W)=0$.

Proof. If $W$ is different from $\mathcal{C}(\{s\} \otimes \alpha)$ this follows from the property (77) of the Green function assigned to the edge decorated by $\{s\}$. The restriction of $\operatorname{Cor}_{\mathcal{H}} \mathcal{C}(\{s\} \otimes \alpha)$ to the divisor $s=s_{0}$ is zero by the very definition. 
The decoration $W_{1}=\{a\} \otimes W_{1}^{\prime}$ delivers an a priori divergent correlator $\operatorname{Cor}_{\mathcal{H}, a}\left(\{a\} \otimes W_{1}^{\prime}\right)$. We understand it via the specialization of a correlator $\operatorname{Cor}_{\mathcal{H}, s_{0}}\left(\{a\} \otimes W_{1}^{\prime}\right)$ when $a \rightarrow s_{0}$ at the tangent vector $v_{0}$ at $s_{0}$. This specialization is zero by Lemma 7.12. This settles the (1b) case.

(1c) The contribution of the reduced Casimir term matches the form assigned by the map $\operatorname{Cor}_{\mathcal{H}}$ to the minus of the sum of forests on Fig [12, The latter sum is the contribution of the edge $E$ to the map $\partial_{\text {Cas }}$.
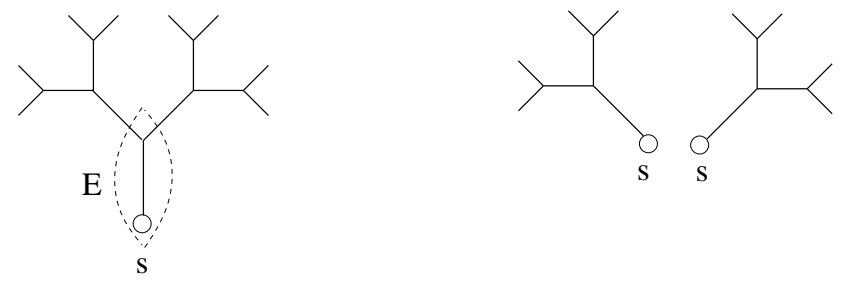

Figure 22: Contribution of the $\delta_{\Delta_{X}}$-term for an external S-decorated edge.

2. $E$ is an external edge decorated by a section $s$. Then there are the following cases.

(2a) The contribution of the $\delta_{\Delta_{X}}$-term in $\Delta G_{E}$ for a tree $T$ is calculated as follows. Let $s$ be the decoration at the external vertex $v_{E}$ of $E$. Let us cut out a small neighborhood of $E$ from $T$, getting connected trees $T_{1}, \ldots, T_{k}$, see Fig 22. Each of them has a new external vertex, and the other external vertices are the ones of $T$ minus $v_{E}$. The tree $T_{i}$ is decorated: the new vertex is decorated by $s$, and the others inherit their decorations from $T$. This matches the component $\partial_{S}$ of the differential $\partial$.

(2b) The contribution of the volume term in $\Delta G_{E}$ is zero. It is similar to (1b), except that one needs to add an argument for the contribution of $\delta_{s_{0}}$ related to an external vertex $v$ of $E$, decorated by $s$. The contribution is $\delta\left(s_{0}-s\right)$. It contributes zero since the divisor $S$ is smooth over $B$, so, moving over $B$, the point $s$ can not collide with $s_{0}$.

(2c) It is similar to (1c). The contribution of the reduced Casimir term in $\Delta G_{E}$ is negative of the form on $X^{\{S \text {-vertices }\}}$ given by the following recepee, illustrated on Fig 16. Cutting the edge $E$, we get a connected tree $T^{\prime}$, and a single edge graph. The latter is decorated by a form $\alpha_{k}^{\vee}$ and $s$. The form $\alpha_{k}^{\vee}$ is viewed as a form on the $s$-copy of $X$. The tree $T^{\prime}$ is decorated: the inherited from $T$ external vertices inherit their decoration, and the new external vertex is decorated by $\alpha_{k}$. It gives rise to a Hodge correlator, which is a form on

$$
X^{\{S \text {-decorated vertices of } T\}-\{s\}} .
$$

The sum over $k$ of the product of this correlator and the form above delivers the desired form.

Denote by $\delta_{\text {Colie }}$ the cocommutator in the Lie coalgebra $\mathcal{C}_{X, S^{*}}(-1)$. 
Corollary 7.13. The following diagram is commutative:

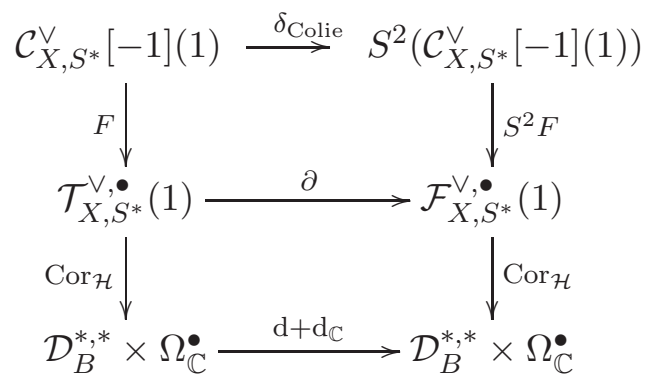

Proof. The bottom square is commutative by Theorem 7.11, the top by Lemma 6.4

By the very definition, the element $\mathbf{G} \in \mathcal{C}_{\mathcal{H}_{\mathbb{R}}}^{1}\left(\mathcal{C}_{X, S^{*}}(-1)\right)$ is obtained by dualising the composition of the left vertical maps.

Recall the Hodge complex differential $\delta_{\mathrm{Hod}}$. Similarly, the element $-\delta_{\mathrm{Hod}} \mathbf{G}$ is obtained by dualising the triple composition:

$$
\mathcal{C}_{X, S^{*}}^{\vee}[-1](1) \stackrel{F}{\longrightarrow} \mathcal{T}_{X, S^{*}}^{\vee}(1) \stackrel{\mathrm{Cor}_{\mathcal{H}}}{\longrightarrow} \mathcal{D}_{B}^{*, *} \otimes \Omega_{\mathbb{C}^{2}}^{\bullet} \stackrel{\mathrm{d}_{B}+\mathrm{d}_{\mathbb{C}}}{\longrightarrow} \mathcal{D}_{B}^{*, *} \otimes \Omega_{\mathbb{C}^{2}}^{\bullet}
$$

By Corollary 17.13, the triple composition coincides with the other triple composition:

$$
\mathcal{C}_{X, S^{*}}^{\vee}[-1](1) \stackrel{\delta_{\text {Colie }}}{\longrightarrow} S^{2}\left(\mathcal{C}_{X, S^{*}}^{\vee}[-1](1)\right) \stackrel{S^{2} F}{\longrightarrow} \mathcal{F}_{X, S^{*}}^{\vee, \bullet}(1) \stackrel{\text { Cor }_{\mathcal{H}}}{\longrightarrow} \mathcal{D}_{B}^{*, *} \otimes \Omega_{\mathbb{C}^{2}}^{\bullet}
$$

By Proposition 7.10 the product $\mathbf{G} \wedge \mathbf{G} \in \mathcal{C}_{\mathcal{H}_{\mathbb{R}}}^{2}\left(\mathcal{C}_{X, S^{*}}(-1)\right)$ is obtained by dualising the latter composition. This implies that $\delta_{\text {Hod }} \mathbf{G}+\mathbf{G} \wedge \mathbf{G}=0$. Theorem 7.8 is proved.

\section{The Lie algebra of special derivations}

The pronilpotent completion $\pi_{1}^{\text {nil }}\left(X-S, v_{0}\right)$ was described in Section 1.2. It is a pro-Lie algebra over $\mathbb{Q}$. It carries a weight filtration. The corresponding associate graded $\mathrm{L}_{X, S}$ has a simple description in terms of the cohomology of $X$ and $S$. We describe the Lie algebra of all special derivations of $\mathrm{L}_{X, S}$, and identify it with the Lie algebra $\mathcal{C}_{X, S}$ from Section 6 .

\subsection{The linear algebra set-up}

Let $H$ be a finite dimensional vector space with a symplectic structure $\omega^{\vee} \in \Lambda^{2} H^{\vee}$. Let $\left\{p_{i}, q_{i}\right\}$ be a symplectic basis in $H: \omega^{\vee}\left(p_{i}, q_{i}\right)=1, \omega^{\vee}\left(p_{i}, p_{j}\right)=\omega^{\vee}\left(q_{i}, q_{j}\right)=0$. There is the dual of $\omega^{\vee}$ :

$$
w=\sum_{i} p_{i} \wedge q_{i} \in \Lambda^{2} H
$$

Let $S$ be a finite set. We need the following associative/Lie algebras:

1. $A_{H, S}$ : the tensor algebra of $H \oplus \mathbb{Q}[S]$. Then $\omega$ provides an element $[p, q]:=\sum_{i}\left[p_{i}, q_{i}\right] \in$ $A_{H, S}$. Denote by $X_{s}$ the generator corresponding to $s \in S$.

2. $L_{H, S}$ : the free Lie algebra generated by $H \oplus \mathbb{Q}[S]$. The algebra $A_{H, S}$ is identified with its universal enveloping algebra. 
3. $\bar{A}_{H, S}$ : the quotient of the algebra $A_{H, S}$ by the two-sided ideal generated by the element

$$
[p, q]+\sum_{s \in S} X_{s}
$$

4. $\bar{L}_{H, S}$ : the quotient of $L_{H, S}$ by the ideal generated by (210).

When $S$ is empty, set $A_{H}:=A_{H, \emptyset}, L_{H}:=L_{H, \emptyset}$, etc.

Let $S$ assume from now on that the set $S$ is non-empty. Let 0 be an element of $S$. Set $S^{*}:=S-\{0\}$. Then there are canonical isomorphisms

$$
L_{H, S^{*}} \stackrel{\sim}{\longrightarrow} \bar{L}_{H, S}, \quad A_{H, S^{*}} \stackrel{\sim}{\longrightarrow} \bar{A}_{H, S} .
$$

Indeed, one can express $X_{0}$ via the other generators:

$$
X_{0}=-\sum_{s \in S^{*}} X_{s}-[p, q] .
$$

Example. Let $X$ be a smooth complex compact curve, $H:=H_{1}(X ; \mathbb{Q})$. The symplectic form $\omega^{\vee}$ is the intersection form on $H_{1}(X ; \mathbb{Q})$. Let $S$ be a non-empty subset of points of $X$. Then $\bar{L}_{H, S}$ is isomorphic, non-canonically, to $\pi_{1}^{\text {nil }}\left(X-S, v_{0}\right)$. The $\bar{L}_{H, S}$ is canonically identified with the associate graded for the weight filtration $\mathrm{L}_{X, S}$ of the $\pi_{1}^{\text {nil }}\left(X-S, v_{0}\right)$.

\subsection{Special derivations of the algebra $\bar{A}_{H, S}$}

Constructions of Section 8.2 generalize the ones of Drinfeld [Dr] and Kontsevich [K]. A derivation $\mathcal{D}$ of the algebra $\bar{A}_{H, S} \stackrel{\sqrt{211}=}{=} A_{H, S^{*}}$ is special if there exist elements $B_{s}, s \in S^{*}$ such that

$$
\mathcal{D}\left(X_{0}\right)=0, \quad \mathcal{D}\left(X_{s}\right)=\left[B_{s}, X_{s}\right], \quad s \in S^{*} .
$$

Special derivations form a Lie algebra denoted $\operatorname{Der}^{S} A_{H, S^{*}}$. Let us emphasize that we define it using the isomorphism (211).

Remark. If $H \neq 0$, a collection of elements $\left\{B_{s}\right\}$ does not determine a special derivation. For instance if $S^{*}$ is empty this collection is also empty while, as we show below, the space of special derivations is not. If $H=0$ it does, see Section 4 in [G4].

Let $A$ be an associative algebra. Recall the projection $\mathcal{C}: A \longmapsto \mathcal{C}(A)=A /[A, A]$.

If $B$ is a free associative algebra generated by a finite set $\mathcal{S}$, then there are linear maps

$$
\partial / \partial X_{s}: \mathcal{C}(B) \longrightarrow B ; \quad \mathcal{C}\left(X_{s_{1}} X_{s_{2}} \cdots X_{s_{k}}\right) \longmapsto \sum_{s_{i}=s} X_{s_{i+1}} X_{s_{i+2}} \cdots X_{s_{i-1}} .
$$

These maps are components of the non-commutative differential map

$$
\mathrm{D}: \mathcal{C}(B) \longrightarrow B \otimes \mathbb{Q}[S], \quad \mathrm{D}(F)=\sum_{s \in S} \frac{\partial F}{\partial X_{s}} \otimes X_{s} .
$$

According to formula (96) in Section 4 of [G4] (an easy exercise) one has

$$
\sum_{s \in S}\left[\frac{\partial F}{\partial X_{s}}, X_{s}\right]=0
$$

In particular, there are linear maps $\partial / \partial p_{i}, \partial / \partial q_{i}, \partial / \partial X_{s}: \mathcal{C}\left(A_{H, S^{*}}\right) \rightarrow A_{H, S^{*}}, s \in S^{*}$. 
Remark. We can not define these maps for $s \in S$ acting on $\bar{A}_{H, S}$ since relation (210) will not be killed.

The vector space $\mathcal{C}\left(A_{H, S^{*}}\right)$ is decomposed into a direct sum of $\mathbb{Q} \cdot 1$ and of $\mathcal{C}^{+}\left(A_{H, S^{*}}\right)$.

Lemma 8.1. There is a map

$$
\kappa: \mathcal{C}^{+}\left(A_{H, S^{*}}\right) \longrightarrow \operatorname{Der}^{S}\left(A_{H, S^{*}}\right) .
$$

such that given an element $F \in \mathcal{C}^{+}\left(A_{H, S^{*}}\right)$, the derivation $\kappa_{F}$ acts on the generators by

$$
X_{0} \longmapsto 0 ; \quad p_{i} \longmapsto-\frac{\partial F}{\partial q_{i}} ; \quad q_{i} \longmapsto \frac{\partial F}{\partial p_{i}} ; \quad X_{s} \longmapsto\left[X_{s}, \frac{\partial F}{\partial X_{s}}\right], \quad s \in S^{*} .
$$

Proof. It follows from (214) that $\sum_{i}\left[\frac{\partial F}{\partial q_{i}}, q_{i}\right]+\sum_{i}\left[\frac{\partial F}{\partial p_{i}}, p_{i}\right]+\sum_{s \in S^{*}}\left[\frac{\partial F}{\partial X_{s}}, X_{s}\right]=0$. This is equivalent to $\kappa_{F}\left([p, q]+\sum_{s \in S^{*}} X_{s}\right)=0$, which just means that $\kappa_{F}\left(X_{0}\right)=0$, and thus $\kappa_{F}$ is a special derivation.

The space $\mathcal{C}\left(A_{H, S^{*}}\right)$ is identified, as a vector space, with the Lie algebra $\mathcal{C}_{H, S^{*}}$ from Section 6. So it inherits a Lie algebra structure.

Proposition 8.2. a) The Lie bracket on $\mathcal{C}\left(A_{H, S^{*}}\right)$ is given by the following formula:

$$
\{F, G\}:=\mathcal{C}\left(2 \sum_{s \in S^{*}}\left[\frac{\partial F}{\partial X_{s}}, \frac{\partial G}{\partial X_{s}}\right] \cdot X_{s}+\sum_{i}\left(\frac{\partial F}{\partial p_{i}} \cdot \frac{\partial G}{\partial q_{i}}-\frac{\partial F}{\partial q_{i}} \cdot \frac{\partial G}{\partial p_{i}}\right)\right) .
$$

b) The map $\kappa$ is a Lie algebra morphism.

Notice that (216) does not depend on the choice of the symplectic basis $\left\{p_{i}, q_{i}\right\}$ in $H$.

Proof. a) Follows immediately from the definitions. The first term in formula (216) corresponds to the map $\delta_{S}$, and the second $\delta_{\text {Cas }}$. In particular this shows that the bracket (216) satisfies the Jacobi identity.

b) We will check it in the more general motivic set-up in Section 10 .

Let $\mathcal{C}^{\prime}\left(A_{H, S^{*}}\right)$ be the quotient of the vector space $\mathcal{C}\left(A_{H, S^{*}}\right)$ by $\oplus_{s \in S^{*}} \mathbb{Q}\left[X_{s}\right]$. The Lie bracket (216) descends to a Lie bracket on $\mathcal{C}^{\prime}\left(A_{H, S^{*}}\right)$,

Proposition 8.3. The map $\kappa^{\prime}: \mathcal{C}^{\prime}\left(A_{H, S^{*}}\right) \longrightarrow \operatorname{Der}^{S} A_{H, S^{*}}$ is a Lie algebra isomorphism.

Proof. Since the algebra $\mathrm{A}_{H, S^{*}}$ is free, the centralizer of $X_{s}$ is the algebra $\mathbb{Q}\left[X_{s}\right]$ of polynomials in $X_{s}$. So Ker $\kappa=\oplus_{s} \mathbb{Q}\left[X_{s}\right]$. We get an injective Lie algebra map $\kappa^{\prime}: \mathcal{C}^{\prime}\left(\mathrm{A}_{H, S^{*}}\right) \hookrightarrow \operatorname{Der}^{S} \mathrm{~A}_{H, S^{*}}$. So it remains to check that $\kappa^{\prime}$ is surjective. We reduce this to the known case when $\operatorname{dim} H=0$. Observe that a system $B=\left(B_{q_{i}}, B_{p_{i}}, B_{s}\right)$ of elements of $A_{S^{*}}$, where $i=1, \ldots, \operatorname{dim} H$ and $s \in S$, satisfying

$$
\left.\sum_{i=1}^{\operatorname{dim} H}\left(\left[B_{q_{i}}, q_{i}\right]+\left[B_{p_{i}}, p_{i}\right]\right)+\sum_{s \in S}\left[B_{s}, X_{s}\right]\right)=0
$$

defines a special derivation $D_{B}$ of the algebra $A_{S^{*}}$, acting on the generators as follows:

$$
D_{B}: p_{i} \longmapsto-B_{q_{i}}, \quad q_{i} \longmapsto B_{p_{i}}, \quad X_{s} \longmapsto B_{s} .
$$

Indeed, $D_{B}\left([p, q]+\sum_{s \in S} X_{s}\right)$ is given by the left hand side of (217). So by Proposition 5.1 of [G4], there exists an $F \in \mathcal{C}\left(A_{S^{*}}\right)$ such that $B_{q_{i}}=-\partial F / \partial q_{i}, B_{p_{i}}=\partial F / \partial p_{i}$, and $X_{s}=\partial F / \partial X_{s}$. 
Just like in (25), the Lie algebra $\mathcal{C} \mathcal{L} i e_{H, S^{*}}$ is a Lie subalgebra of $\mathcal{C}\left(A_{H, S^{*}}\right)$, defined as follows:

$$
\mathcal{C} \mathcal{L} i e_{X, S^{*}}^{\vee}:=\frac{\mathcal{C}\left(\mathrm{A}_{H, S^{*}}^{\vee}\right)}{\text { Shuffle relations }} ; \quad \mathcal{C} \mathcal{L} i e_{H, S^{*}}:=\text { the dual of } \mathcal{C} \mathcal{L} i e_{H, S^{*}}^{\vee}
$$

Proposition 8.4. The map $\kappa: \mathcal{C} \mathcal{L}_{i} e_{H, S^{*}} \longrightarrow \operatorname{Der}^{S} L_{H, S^{*}}$ is a Lie algebra isomorphism.

Proof. Let us consider a Casimir element, given by a formal infinite sum

$$
\xi:=\sum_{W} \frac{1}{|\operatorname{Aut}(W)|} W^{\vee} \otimes W .
$$

Here the sum is over a basis $\{W\}$ in $\mathcal{C}_{H, S^{*}}$, and $\left\{W^{\vee}\right\}$ is the dual basis. Here $\operatorname{Aut}(W)$ is the automorphism group of the cyclic word $W$. There is a coproduct $\Delta$ on the algebra $\mathrm{A}_{H, S^{*}}$, making it into a Hopf algebra, determined by the property that the generators of the algebra are primitive. It dualizes the shuffle product ${ }^{{ }_{\text {Sh }}}$. Let $\bar{\Delta}(Z):=\Delta(Z)-(1 \otimes Z+Z \otimes 1)$ be the reduced coproduct. Then $\mathrm{L}_{H, S^{*}}=\operatorname{Ker} \bar{\Delta}$. Choose a basis $\left\{Y_{i}\right\}$ in $\mathrm{V}_{H, S^{*}}$. Given a basis vector $Y_{i}$, consider the expression

$$
\mathrm{id} \otimes\left(\bar{\Delta} \circ \mathcal{D}_{Y_{i}}(\xi)\right) \in \mathcal{C}_{H, S^{*}}^{\vee} \otimes \mathrm{A}_{H, S^{*}}
$$

Choose two elements $A, B$ of the induced basis in $\mathrm{A}_{H, S^{*}}$. Then $A \otimes B \in \mathrm{A}_{H, S^{*}} \otimes \mathrm{A}_{H, S^{*}}$ appears in (219) with coefficient $\mathcal{C}\left(Y\left(A \circ_{\mathrm{Sh}} B\right)\right)$. Notice that the factor $1 /|\operatorname{Aut}(W)|$ is necessary.

\subsection{A symmetric description of the Lie algebra of special derivations}

To define a special derivation we have to choose a specific element, 0 , of the set $S$. Let us show that the Lie algebra of all special derivations can be defined using only the symplectic vector space $H$ and the set $S$. However the way it acts by special derivations of $A_{H, S^{*}}$ depends on the choice of $0 \in S$.

Let us define a Lie algebra structure on the vector space $\mathcal{C}\left(A_{H, S}\right)$ via formula (216) where $S^{*}$ is replaced by $S$. Then we prove the following result.

Proposition 8.5. Take the two-sided ideal in the algebra $A_{H, S}$, generated by $[p, q]+\sum_{s \in S} X_{s}$. Its image in the Lie algebra $\mathcal{C}\left(A_{H, S}\right)$ is an ideal. So the quotient $\mathcal{C}\left(\bar{A}_{H, S}\right)$ of $\mathcal{C}\left(A_{H, S}\right)$ by this ideal is a Lie algebra. The canonical map $i_{0}: \mathcal{C}\left(\bar{A}_{H, S}\right) \longrightarrow \mathcal{C}\left(A_{H, S^{*}}\right)$ is a Lie algebra isomorphism.

Proof. It is an easy exercise left to the reader 8

So for any element $s \in S$ there is a Lie algebra isomorphism

$$
\kappa_{s}: \mathcal{C}\left(\bar{A}_{H, S}\right) \stackrel{\sim}{\longrightarrow} \operatorname{Der}^{S} A_{H, S-\{s\}}
$$

The isomorphism $i_{s}: A_{H, S-\{s\}} \rightarrow \bar{A}_{H, S}$ provides a Lie algebra isomorphism $\mathcal{C}\left(A_{H, S-\{s\}}\right) \rightarrow$ $\mathcal{C}\left(\bar{A}_{H, S}\right)$. Furthermore, for any $s \in S$ isomorphism (220) restricts to a Lie algebra isomorphism

$$
\kappa_{s}: \mathcal{C} \mathcal{L} i e\left(\bar{L}_{H, S}\right) \stackrel{\sim}{\longrightarrow} \operatorname{Der}^{S} L_{H, S-\{s\}} .
$$

There is a Lie algebra isomorphism $\mathcal{C} \mathcal{L} i e\left(L_{H, S-\{s\}}\right) \rightarrow \mathcal{C}\left(\bar{L}_{H, S}\right)$.

\footnotetext{
${ }^{8} \mathrm{~A}$ natural proof of is given by deducing it from a general result in the DG version of the story.
} 
The Lie algebra of outer semi-special derivations. We say that a derivation $\mathcal{D}$ of the associative algebra $\bar{A}_{H, S}$ is semi-special if it preserves the conjugacy classes of each of the generators $X_{s}, s \in S$, i.e. if there exist elements $B_{s}$ of the algebra such that

$$
\mathcal{D}\left(X_{s}\right)=\left[B_{s}, X_{s}\right], \quad s \in S .
$$

The Lie algebra of semi-special derivations is denoted by $\operatorname{Der}^{S S} \bar{A}_{H, S}$. An inner derivation is a particular example of a semi-special derivation. We define the Lie algebra of outer semispecial derivations of the algebra $\bar{A}_{H, S}$ by taking the quotient modulo the Lie subalgebra of inner derivations:

$$
\operatorname{ODer}^{S S} \bar{A}_{H, S}:=\frac{\operatorname{Der}^{S S} \bar{A}_{H, S}}{\operatorname{InDer}^{S S} \bar{A}_{H, S}} .
$$

For an $s \in S$, let $\operatorname{Der}_{s}^{S} \bar{A}_{H, S} \subset \operatorname{Der}^{S S} \bar{A}_{H, S}$ be the Lie subalgebra of all semi-special derivations which are special for the generator $X_{s}$, i.e. kill $X_{s}$. Thus there is a Lie algebra map

$$
p_{s}: \operatorname{Der}_{s}^{S} \bar{A}_{H, S} \longrightarrow \operatorname{ODer}^{S S} \bar{A}_{H, S}
$$

Lemma 8.6. The map $p_{s}$ is surjective. Its kernel is spanned by the inner derivation $\left[X_{s}^{n}, *\right]$.

Proof. Let $\mathcal{D}$ be a semi-special derivation, such that $\mathcal{D}\left(X_{s}\right)=\left[B_{s}, X_{s}\right]$. Subtracting the inner derivation $\left[B_{s}, *\right]$ from $\mathcal{D}$ we get a derivation special with respect to the generator $X_{s}$. So $p_{s}$ is surjective. Since $\bar{A}_{H, S-\{s\}}$ is a free associative algebra, we get the second claim.

\section{$9 \quad$ Variations of mixed $\mathbb{R}-H o d g e$ structures via Hodge correlators}

A variation of $\mathbb{R}$-MHS on $\pi^{\text {nil }}\left(X-S, v_{0}\right)$ via Hodge correlators. Recall that $S=S^{*} \cup\left\{s_{0}\right\}$. Recall (Section 1.10) the enhanced moduli space $\mathcal{M}_{g, n}^{\prime}$ parametrising collections $S$ of $n$ distinct points on a genus $g$ complex curve $X$ plus a tangent vector at $s_{0}$. There is a local system $\mathcal{L}$ over $\mathcal{M}_{g, n}^{\prime}$ with the fiber

$$
\mathrm{L}_{X, S^{*} ; v_{0}} \cong \mathrm{gr}^{W} \pi_{1}^{\mathrm{nil}}\left(X-S, v_{0}\right) .
$$

It is equipped with the Gauss-Manin connection.

A tangent vector $v_{0}$ at the point $s_{0} \in X$ provides the normalized Green function $G_{v_{0}}(x, y)$ (Section 3.11). It gives rise to the Hodge correlator map, and hence to an element

$$
\widetilde{\mathbf{G}}_{v_{0}} \in \mathcal{C}_{\mathcal{H}}^{1}\left(\mathcal{C} \mathcal{L} i e_{X, S}\right) .
$$

The Lie algebra $\mathcal{C}_{X, S}$ acts by special derivations of the Lie algebra $\mathrm{L}_{X, S^{*} ; v_{0}}$ (Section 8 ). So we arrive at an endomorphism $\mathbf{G}_{v_{0}}$ of the smooth bundle $\mathcal{L}_{\infty}$. We proved in Section 7 that it satisfies the Maurer-Cartan differential equation $\delta \mathbf{G}_{v_{0}}+\left[\mathbf{G}_{v_{0}}, \mathbf{G}_{v_{0}}\right]=0$. Therefore the construction of Section 5 provides a variation of mixed $\mathbb{R}$-Hodge structures. Let us recall Theorem 1.12 .

Theorem 9.1. When the data $\left(X, S, v_{0}\right)$ varies, the variation of mixed $\mathbb{R}$-Hodge structures given by the Green datum $\mathbf{G}_{v_{0}}$ is isomorphic to the classical variation of the mixed $\mathbb{R}$-Hodge structures on $\pi_{1}^{\text {nil }}\left(X-S, v_{0}\right)$. 
A simple proof of Theorem 9.1 for rational curves. Denote by $G_{p, p}^{\text {Cor }}$ the Green operators for the Hodge correlators on $\mathbb{P}^{1}-S$. The standard variation of mixed $\mathbb{R}$-Hodge structures $\pi_{1}^{\mathcal{H}}\left(\mathbb{P}^{1}-S, v_{0}\right)$ is described by the Green operators denoted by $G_{p, p}^{\text {st }}$. Let us prove by induction on $p$ that $G_{p, p}^{\text {Cor }}=G_{p, p}^{\text {st }}$. The base of the induction is given by the following straightforward Lemma:

Lemma 9.2. One has $G_{1,1}^{\mathrm{Cor}}=G_{1,1}^{\mathrm{st}}$.

Suppose that the claim is proved for $p<n$. The systems of differential equations for Green operators describing variations of $\mathbb{R}-$ MHS implies that the endomorphisms $G_{n, n}^{\text {Cor }}$ and $G_{n, n}^{\text {st }}$ satisfy the same differential equations, and that $G_{n, n}^{\text {Cor }}-G_{n, n}^{\text {st }}$ is annihilated by both $\partial$ and $\bar{\partial}$, and thus is a constant. To check that this constant is zero we use the shuffle relations from Proposition 3.5. which imply that a non-zero multiple of this constant is zero. This proves the theorem.

This argument does not work in the non-Tate case since we do not impose $\partial$-differential equations on $G_{1, q}$, as well as $\bar{\partial}$-differential equations on $G_{p, 1}$. Let us address now the general case of Theorem 1.12 ,

$\mathbb{R}$-MHS on $\pi_{1}^{\text {nil }}(X-S, a)$ with a regular base point a via Hodge correlators. Let $a \in X-S$. Choose a non-zero tangent vector $v_{a}$ at $a$. There is a canonical isomorphism

$$
\pi_{1}^{\mathrm{nil}}(X-S, a)=\pi_{1}^{\mathrm{nil}}\left(X-S \cup\{a\}, v_{a}\right) /\left(v_{a}\right)
$$

where $\left(v_{a}\right)$ is the ideal generated by $v_{a}$. The Green operator on $\operatorname{gr}^{W} \pi_{1}^{\mathrm{nil}}\left(X-S \cup\{a\}, v_{a}\right)$ is a special derivation, and thus preserves the ideal $\operatorname{gr}^{W}\left(v_{a}\right)$. So it induces an operator $\mathbf{G}_{v_{a}}$ on the quotient, which gives rise to an $\mathbb{R}-\mathrm{MHS}$ on $\pi_{1}^{\mathrm{nil}}(X-S, a)$.

Good unipotent variations. R. Hain and S. Zucker [HZ introduced a notion of a good unipotent variation of mixed Hodge structures on an open algebraic variety $U$, and proved that the category of such variations is equivalent to the category of representations $\pi_{1}^{\text {nil }}(U, u) \otimes V \longrightarrow$ $V$ which are morphism of mixed Hodge structures. Equivalently, they are representations in the category mixed Hodge structures of the universal enveloping algebra $\mathrm{A}^{\mathcal{H}}(U, u)$ of $\pi_{1}^{\text {nil }}(U, u)$ with the induced mixed Hodge structures.

Recall ([H1], Section 7) that a unipotent variation of mixed Hodge structures $\mathcal{V}$ over a smooth complex curve $X=\bar{X}-D$, where $\bar{X}$ is a smooth compactification, and $D$ a finite number of points, is a good unipotent one if the following conditions at infinity hold:

- (i) Let $\overline{\mathcal{V}} \rightarrow \bar{X}$ be Deligne's canonical extension of the local system $\mathcal{V}$. The Hodge bundles $F^{p} \mathcal{V}$ are required to extend to holomorphic subbundles $F^{p} \overline{\mathcal{V}}$ of $\overline{\mathcal{V}}$.

- (ii) Let $N_{P}:=\frac{1}{2 \pi i} \log T_{P}$ be the logarithm of the local monodromy $T_{P}$ around $P \in D$. Then

$$
N_{P}\left(W_{l} V_{x}\right) \subset W_{l-2} V_{x}
$$

The second condition is so simple because we assumed that the global monodromy is unipotent.

Theorem 9.3. The variation $\mathcal{L}$ of $\mathbb{R}-M H S$ on $\pi_{1}^{\text {nil }}(X-S, a)$ defined by the operator $\mathbf{G}_{v_{a}}$ acting on (222), is a good unipotent variation of mixed Hodge structures over $a \in X-S$.

We introduce a DGA of forms with logarithmic singularities, and use it to prove Theorem 9.3. Then we show how Theorem 9.3 implies Theorem 1.12 . 
Differential forms on $X$ with tame logarithmic singularities. Let $D$ be a normal crossing divisor in a smooth complex projective variety $\bar{X}$, and $X:=\bar{X}-D$. Let us define a DG subalgebra $\mathcal{A}_{\log }(X)$ of differential forms on $X$ with tame logarithmic singularities on $X$. It is a slight modification of the space used in 1.3 .10 in $[\mathrm{L}$. It is a DG subalgebra of the de Rham DG algebra of smooth forms on $X$. Choose local equations $z_{i}$ of the irreducible components $D_{i}$ of the divisor $D$. The space $\mathcal{A}_{\log }(X)$ consists of forms $\omega$ which can be represented as polynomials in $\log \left|z_{i}\right|, \partial \log \left|z_{j}\right|$, and $\bar{\partial} \log \left|z_{k}\right|$,

$$
\omega=\sum_{a_{i}, \varepsilon_{j}^{\prime}, \varepsilon_{k}^{\prime \prime}} \omega_{a_{i}, \varepsilon_{j}^{\prime}, \varepsilon_{k}^{\prime \prime}} \log ^{a_{i}}\left|z_{i}\right| \wedge \bigwedge_{j}\left(\partial \log \left|z_{j}\right|\right)^{\varepsilon_{j}^{\prime}} \wedge \bigwedge_{k}\left(\bar{\partial} \log \left|z_{k}\right|\right)^{\varepsilon_{k}^{\prime \prime}}
$$

whose coefficients are smooth functions on $\bar{X}$ with the following property: a coefficient of a monomial containing (in positive degree) any of the three expressions $\log \left|z_{i}\right|, \partial \log \left|z_{i}\right|$, and $\bar{\partial} \log \left|z_{i}\right|$ related to the stratum $D_{i}$ vanishes at the stratum $D_{i}$. For example, $z_{1} \log \left|z_{1}\right|$ belongs $\mathcal{A}_{\log }(X)$, while $z_{1} \log \left|z_{2}\right|$ does not. The space $\mathcal{A}_{\log }(X)$ clearly does not depend on the choice of local equations $z_{i}$. The space $\mathcal{A}_{\log }(X)$ is closed under $\partial$ and $\bar{\partial}$.

Proposition 9.4. Choose a local parameter $t$ at $s$ such that dt is dual to the tangent vector $v_{s}$ at s. Then $\mathbf{G}_{v_{a}}-\log t \cdot \operatorname{ad}\left(X_{s}\right)$ is a smooth function with tame logarithmic singularities near the point $s$.

Corollary 9.5. The logarithm of the monodromy of the local system $\mathcal{L}$ around $s$ is $\operatorname{ad}\left(X_{s}\right)$.

Proof. Indeed, $d^{\mathbb{C}} \log |t|=i d \arg (t)$, and $d^{\mathbb{C}}$ of the other components of $\mathbf{G}_{v_{a}}$ vanish at $t=0$ since these components have tame logarithmic singularities at $t=0$.

We prove Proposition 9.4 later on. Let us show first how it implies Theorems 9.3 and 1.12

Proof of Theorem 9.3. The associate graded $\operatorname{gr}^{W} \mathcal{L}$ is a constant variation. So the local system $\mathcal{L}$ is unipotent. Thus we must check only conditions (i) and (ii).

Condition (ii) follows immediately from Corollary 9.5.

Condition (i) is just a condition on the Hodge filtration on the local system on $X$-it does not involve the weight filtration. So, using flatness of the twistor connection, it is sufficient to prove it for the twistor connection $\nabla_{\mathcal{G}}^{(1)}$ restricted to $u=1$ instead of $u=0$. The Hodge filtration for the connection $\nabla_{\mathcal{G}}^{(1)}$ is constant. This implies the claim. Theorem 9.3 is proved.

Proof of Theorem 9.1. By Theorem 9.3 there is a good unipotent variation of mixed $\mathbb{R}$ Hodge structures on $X-S$ given by the universal enveloping algebras $\mathrm{A}(X-S, a)$ of (222), when $a$ varies. By the Hain-Zucker theorem [HZ], it is described by a morphism of $\mathbb{R}-\mathrm{MHS}$

$$
\theta: \mathrm{A}^{\mathcal{H}}(X-S, a) \otimes \mathrm{A}(X-S, a) \longrightarrow \mathrm{A}(X-S, a) .
$$

Observe that $\mathrm{A}(X-S, a)$ is a free rank one $\mathrm{A}^{\mathcal{H}}(X-S, a)$-module with a canonical generator given by the unit $e \in \mathrm{A}(X-S, a)$.

Lemma 9.6. The action $\theta$ coincides with the standard one. 
Proof. The action $\theta$ is a morphism of mixed $\mathbb{R}$-Hodge structures. We understand them as bigraded objects equipped with the action of the Hodge Galois Lie algebra. In particular $\theta$ preserves the bigrading and hence the weight.

The action $\theta$ is determined by the images of the generators. The latter are operators of weight -1 or -2 . The weight -1 generators are determined by the weight -1 component of the connection $\nabla_{\mathcal{G}}^{0}$ on $\mathcal{L}_{\infty}$. Indeed, the weight -1 component of the parallel transform is determined by the weight -1 component of the connection, and the same is true for the weight -1 component of the exponential of the action of the generators. The weight -1 component of our connection is given by the canonical 1-form $\nu$ (Section 2.2), and the same is true for the standard one. Thus the weight -1 generators act in the standard way.

Corollary 9.5 implies the claim for the weight -2 generators $X_{s}$.

The Hodge Galois group acts by automorphisms, and hence acts trivially on the unit $e$. So there is an isomorphism of mixed $\mathbb{R}$-Hodge structures $\mathrm{A}^{\mathcal{H}}(X-S, a) \otimes e \stackrel{\sim}{\longrightarrow} A(X-S, a)$. Theorem 1.12 is proved.

The proof of Proposition 9.4 is a bit technical. Here is a crucial step.

Dependence of $\mathbf{G}_{v_{a}}$ on $a$. Below the point $s$, a puncture on the curve, is fixed. Let us calculate the part of $\mathbf{G}_{v_{a}}$ depending on the point $a$, and investigate what happens when $a$ approaches to a puncture $s$. Recall (76) that

$$
G_{a}\left(x_{1}, x_{2}\right)=G_{\mathrm{Ar}}\left(x_{1}, x_{2}\right)-G_{\mathrm{Ar}}\left(a, x_{2}\right)-G_{\mathrm{Ar}}\left(x_{1}, a\right) .
$$

Lemma 9.7. Assume that none of the vertices of an edge $E$ of a decorated tree $T$ is as on Fig 23, i.e. either $S$-decorated or decorated by a pair of 1-forms. Then replacing the Green function $G_{v_{a}}$ assigned to $E$ by $G_{\mathrm{Ar}}\left(x_{1}, x_{2}\right)$ we do not change the Hodge correlator integral assigned to $T$.

Proof. Let $v_{1}, v_{2}$ be the vertices of $E$. Each of them is a 3 -valent vertex. Denote by $x_{1}, x_{2}$ the points on the curves assigned to them. Let us assign the function $G_{\text {Ar }}\left(x_{1}, a\right)$ to the edge $E$. Then the integral over $x_{2}$ is proportional to one of the following:

$$
\int_{X} d^{\mathbb{C}} G_{a}\left(y, x_{2}\right) \wedge d^{\mathbb{C}} G_{a}\left(z, x_{2}\right), \quad \text { or } \quad \int_{X} d^{\mathbb{C}} G_{a}\left(y, x_{2}\right) \wedge \alpha .
$$

Each of them is zero: we integrate $d^{\mathbb{C}}$-exact forms. Similarly for $G_{\mathrm{Ar}}\left(a, x_{2}\right)$.

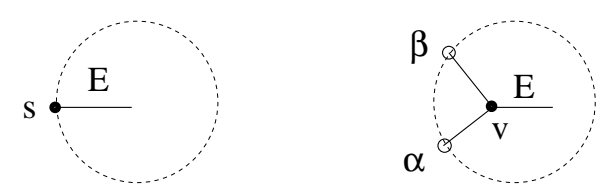

Figure 23: Changing the Green function assigned to the edge $E$ may alter the Hodge correlator.

Let $\left\{\alpha_{i}, \alpha_{j}^{\vee}\right\}$ be a Darboux basis in the symplectic space $\Omega_{X}^{1} \oplus \bar{\Omega}_{X}^{1}$, so that $\int_{X} \alpha_{i} \wedge \alpha_{j}^{\vee}=\delta_{i j}$. Let $\left\{h_{i}, h_{j}^{\vee}\right\}$ be the dual basis in $H_{1}(X, \mathbb{C})$. Then the Hodge correlator is given by

$$
\mathbf{G}_{a}=\sum_{W} \frac{1}{|\operatorname{Aut}(W)|} \operatorname{Cor}_{\mathcal{H}, a}\left(\beta_{1} \otimes \ldots \otimes \beta_{m}\right) \cdot \mathcal{C}\left(h_{\beta_{1}} \otimes \ldots \otimes h_{\beta_{m}}\right), \quad \beta_{i} \in\left\{\alpha_{i}, \alpha_{i}^{\vee}\right\}
$$


The sum is over a basis $W=\mathcal{C}\left(\beta_{1} \otimes \ldots \otimes \beta_{m}\right)$ in the cyclic envelope of the tensor algebra of $H^{1}(X, \mathbb{C})$, and $\left\{h_{\beta}\right\}$ is the dual basis to the basis $\{\beta\}$.

Contribution of an edge $E$ as on Fig 23. Let us calculate the depending on the point $a$ contribution to the integral of an edge $E$ as on Fig 23. Take a tree $T$ with such an edge $E$. Cut out the "small" tree growing from $E$, getting a new tree. Let $W$ be its decoration inherited from $T$. For example, the decoration of the left tree on Fig 23 is $\mathcal{C}(s \otimes W)$. We assert that the depending on $a$ part of the contribution of the edge $E$ to the sum (223) is

$$
\operatorname{Cor}_{\mathcal{H}, \operatorname{Ar}}(\{a\} \otimes W) \cdot \mathcal{C}\left(X_{a} \otimes W^{\vee}\right),
$$

where $W^{\vee}$ is the cyclic word in $H_{1}$ dual to the cyclic words $W$ in $H^{1}$. Indeed, take first the tree on the right of Fig 23, and assign to the edge $E$ the Green function $G_{\operatorname{Ar}}(a, y)$. Then its contribution to the integral is

$$
\operatorname{Cor}_{\mathcal{H}, \operatorname{Ar}}(\{a\} \otimes W) \int_{X} \alpha \otimes \beta
$$

Substituting basis elements $\alpha_{i}, \alpha_{j}^{\vee}$ for $\alpha$ as well as for $\beta$, and taking the sums over $i, j$, we get

$$
\operatorname{Cor}_{\mathcal{H}, A r}(\{a\} \otimes W) \cdot \mathcal{C}\left(\sum_{i}\left[h_{i}, h_{i}^{\vee}\right] \otimes W^{\vee}\right)
$$

Similarly, the tree on the left on Fig 23 contributes

$$
\operatorname{Cor}_{\mathcal{H}, \operatorname{Ar}}(\{a\} \otimes W) \cdot \mathcal{C}\left(\sum_{s \in S} X_{s} \otimes W^{\vee}\right) .
$$

Since $X_{a}=\sum_{s \in S} X_{s}+\sum_{i}\left[h_{i} \cdot h_{i}^{\vee}\right]$, the total contribution is $\operatorname{Cor}_{\mathcal{H}, \operatorname{Ar}}(\{a\} \otimes W) \cdot \mathcal{C}\left(X_{a} \otimes W^{\vee}\right)$.

Remark. A similar argument shows that the Green operator induced on $\mathrm{L}\left(X-\{a\}, v_{a}\right) /\left(v_{a}\right)$ does not depend on the choice of a constant in the Green function $G_{a}(x, y)$. Indeed, we may assume that an edge $E$ as on Fig 23 contributes to the integral of the Green function but not its derivative. Then adding a constant to $G_{a}(x, y)$ amounts to changing a factor in (223) by

$$
\sum_{s \in S} X_{s}+\sum_{i=1}^{g}\left[h_{i}, h_{i}^{\vee}\right]=0 .
$$

Conclusion. Since we may have several edges like on Fig 23, the depending on $a$ part of $\mathbf{G}_{v_{a}}$ is a sum of expressions

$$
\operatorname{Cor}_{\mathcal{H}, \mathrm{Ar}}\left(\{a\} \otimes W_{1} \otimes \ldots \otimes\{a\} \otimes W_{m}\right) \cdot \mathcal{C}\left(X_{a} \otimes W_{1}^{\vee} \otimes \ldots \otimes X_{a} \otimes W_{m}^{\vee}\right) .
$$

Proof of Proposition 9.4. Denote by $\mathbf{G}_{p, q}$ the $(p, q)$-component of $\mathbf{G}_{v_{a}}$.

Let us investigate $\mathbf{G}_{1,1}$. There are only two cyclic words contributing to the depending on $a$ part of $\mathbf{G}_{1,1}: W^{\prime}=\mathcal{C}(\{a\} \otimes\{s\})$ and $W^{\prime \prime}=\mathcal{C}(\{a\} \otimes \alpha \otimes \bar{\beta})$. The first appears with the coefficient given by the Green function $G_{\mathrm{Ar}, \mathrm{va}_{\mathrm{a}}}(a, s)$, and has the singularity $\log t$. It delivers the singular term $\log t \cdot \operatorname{ad}\left(X_{s}\right)$. The second does not depend on $s$. So the claim about $\mathbf{G}_{1,1}$ follows. 
Let us show that $\mathbf{G}_{p, q} \in \mathcal{A}_{\log }(X)$ for $(p, q)=(1, q),(p, 1),(2,2)$ provided that $(p, q) \neq(1,1)$. After this the Lemma follows immediately by the induction using the differential equations on $\mathbf{G}_{p, q}$, see (208). Notice that since $\overline{\mathbf{G}}_{1, q}=-\mathbf{G}_{q, 1}$, the claim about $\mathbf{G}_{1, q}$ implies the one about $\mathbf{G}_{q, 1}$.

Let us prove that $\mathbf{G}_{1, q} \in \mathcal{A}_{\log }(X)$ for $q>1$. The only cyclic words contributing to the depending on $a$ part of $\mathbf{G}_{1, q}$ are $\mathcal{C}\left(\{a\} \otimes\{s\} \otimes \bar{\alpha}_{1} \otimes \ldots \otimes \bar{\alpha}_{q-1}\right)$, where $\bar{\alpha}_{i}$ are antiholomorphic 1 -forms on $\bar{X}$. The crucial case is $q=2$. Let us show that $\operatorname{Cor}_{\mathcal{H}}(\{a\} \otimes\{s\} \otimes \bar{\alpha}) \in \mathcal{A}_{\log }(X)$. We have to investigate the behavior of the following integral at $a \rightarrow s$ :

$$
\int_{\bar{X}} G(a, x) \partial G(s, x) \wedge \bar{\alpha} .
$$

Choose a small neighborhood $U_{s}$ of $s$. We may assume that it contains $a$. Then the integral over its complement is clearly a smooth function in $a$. So we have to show that the integral over $U_{s}$, as a function of $a \in U_{s}$, has a tame logarithmic singularity at $a=s$. The problem is local. So it is sufficient to prove it for a single differential form $\bar{\alpha}$ in $U_{s}$ which does not vanish at $s$. Since $G(0, x)$ has singularity of type $\log |x|$ near $x=0$, and since $\bar{\alpha} \sim \bar{\partial} \log |x-1|$ near $x=0$, we may assume $U_{s}$ is a small neighborhood of 0 in $\mathbb{C}$, and consider an integral

$$
\int_{\mathbb{C P}^{1}} \log |x| \partial \log |x-a| \wedge \bar{\partial} \log |x-1| .
$$

This integral equals to a non-zero multiple of of the Bloch-Wigner dilogarithm function $\mathcal{L}_{2}(a)$, see Section 10.1.2, which is independent of this Section. Since

$$
d \mathcal{L}_{2}(a)=\log |1-a| d \arg a-\log |a| d \arg (1-a),
$$

it has a tame logarithmic singularity at $a=0$. This implies the claim. The claim for $q>2$ follows easily from the $q=2$ case.

Finally, $\mathbf{G}_{2,2}$ is given by either $\operatorname{Cor}_{\mathcal{H}}(\{a\} \otimes\{s\} \otimes \bar{\alpha} \otimes \beta)$, which reduces to the previous case, or by $\operatorname{Cor}_{\mathcal{H}}(\{a\} \otimes\{s\} \otimes \otimes\{x\})$, which is completely similar to the $\mathbf{G}_{1,2}$ case, and done by the reduction to the dilogarithm. Proposition 9.4 is proved.

\section{Motivic correlators on curves}

In Section 8 we described the Lie algebra of special derivations in the Betti realization. In Section 10 we present it in the motivic set-up. Using this we define certain elements of the motivic Lie coalgebra, motivic correlators on curves. Section 9 implies that the Hodge correlators on curves are their periods.

Let $X$ be an irreducible regular projective curve over a field $F$, and $S$ a finite subset of $X(F)$. We explore the motivic fundamental Lie algebra $\mathrm{L}\left(X-S, v_{0}\right)$ of the curve $X-S$ with a tangential base point $v_{0}$ over $F$.

We start by describing several different frameworks: Hodge, $l$-adic, motivic, etc. In each of them we supposed to have a Lie algebra in the corresponding category. It is equipped with the weight filtration $W_{\bullet}$, and $\operatorname{gr}^{W} \mathrm{~L}\left(X-S, v_{0}\right)$ is expressed via $\mathrm{gr}^{W} H^{1}(X-S)$.

The $l$-adic realization $\mathrm{L}\left(X-S, v_{0}\right)$ is an $l$-adic pro-Lie algebra $\mathrm{L}^{(l)}\left(X-S, v_{0}\right)$ - the Lie algebra of the pro-l completion $\pi_{1}^{(l)}\left(X-S, v_{0}\right)$ of the fundamental group. The Galois group of $F$ acts by automorphisms of $\pi_{1}^{(l)}\left(X-S, v_{0}\right)$, and hence by derivations of the Lie algebra $\mathrm{L}^{(l)}\left(X-S, v_{0}\right)$, preserving some data, mostly related to the set $S$. We call such derivations special. 


\subsection{The motivic framework}

Let $F$ be a field. Below we work in one of the following five categories $\mathcal{C}$ :

1. Motivic. The hypothetical abelian category of mixed motives over a field $F$.

2. Hodge. $F=\mathbb{C}$, and $\mathcal{C}$ is the category of mixed $\mathbb{Q}$ - or $\mathbb{R}$-Hodge structures.

3. Mixed l-adic. $F$ is a field such that $\mu_{l} \notin \notin F$, and $\mathcal{C}$ is the mixed category of $l$-adic $\operatorname{Gal}(\bar{F} / F)-$ modules with a filtration $W$. indexed by integers, such that $\operatorname{gr}_{n}^{W}$ is a pure of weight $n$.

4. Motivic Tate. $F$ is a number field, $\mathcal{C}$ is the abelian category of mixed Tate motives over $F$, equipped with the Hodge and $l$-adic realization functors, c.f. DG].

5. Variations of mixed Hodge structures. $F=\mathbb{C}$, and $\mathcal{C}$ is the category of mixed $\mathbb{Q}$ - or $\mathbb{R}$-Hodge structures over a base $B$.

The setup 1) is conjectural. The other four are well defined. A category $\mathcal{C}$ from the list is an $K$-category, where $K=\mathbb{Q}$ in 1 ), 4$), 5) ; K=\mathbb{Q}$ or $\mathbb{R}$ in 2); and $K=\mathbb{Q}_{l}$ in 3).

Each of the categories has an invertible object, the Tate object, which is denoted, abusing notation, $\mathbb{Q}(1)$ in all cases, although $\operatorname{Hom}_{\mathcal{C}}(\mathbb{Q}(1), \mathbb{Q}(1))=K$. So, for instance, in the setup iii) $\mathbb{Q}(1)$ denotes the Tate module $\mathbb{Q}_{l}(1)$. We set $\mathbb{Q}(n):=\mathbb{Q}(1)^{\otimes n}$.

Each object in $\mathcal{C}$ carries a canonical weight filtration $W_{\bullet}$, morphisms in $\mathcal{C}$ are strictly compatible with this filtration. The weight of $\mathbb{Q}(1)$ is -2 .

An object $M$ of $\mathcal{C}$ is pure if $\operatorname{gr}_{n}^{W} M$ is zero for all $n$ but possibly one. Tensor powers and direct summands of pure objects are pure. Let $M$ be a pure object in $\mathcal{C}$. The pure subcategory of $\mathcal{C}$ generated by $M$ is the smallest subcategory containing $M$, and closed under operations of taking tensor products, duals, finite sums and direct summands. The mixed subcategory of $\mathcal{C}$ generated by $M$ is the smallest subcategory of $\mathcal{C}$ closed under extensions and containing the pure subcategory generated by $M$.

Let us briefly recall some features of the Tannakian formalism in the setup of mixed categories which is used below, see [G3] for the proofs. See also [G7] for the mixed Tate case.

There is a canonical fiber functor to the pure tensor category $\mathcal{P}^{\mathcal{C}}$ of pure objects in $\mathcal{C}$ :

$$
\omega: \mathcal{C} \longrightarrow \mathcal{P}^{\mathcal{C}}, \quad X \longmapsto \oplus_{n} \operatorname{gr}_{n}^{W} X .
$$

The ind-object $\operatorname{End}^{\otimes}(\omega)$ of endomorphisms of this functor respecting the tensor product has a natural structure of a Hopf algebra in the tensor category $\mathcal{P}^{\mathcal{C}}$. Let $\mathcal{A}_{\bullet}(\mathcal{C})$ be its graded dual. It is a commutative graded Hopf algebra. The functor $\omega$ provides a canonical equivalence between the category $\mathcal{C}$ and the category of graded $\mathcal{A}_{\bullet}(\mathcal{C})$-comodules in the category $\mathcal{P}^{\mathcal{C}}$. There is the a Lie coalgebra

$$
\mathcal{L}_{\bullet}(\mathcal{C})=\frac{\mathcal{A}_{\bullet}(\mathcal{C})}{\mathcal{A}_{>0}(\mathcal{C})^{2}}
$$

with the cobracket induced by the coproduct in $\mathcal{A}_{\bullet}(\mathcal{C})$. Let $\mathrm{L}_{\bullet}(\mathcal{C})$ be the dual Lie algebra.

Definition 10.1. Let $X$ be a regular projective curve over $F$. Then $\mathcal{P}_{X}$ (respectively $\mathcal{C}_{X}$ ) is the subcategory of $\mathcal{P}^{\mathcal{C}}$ (respectively $\mathcal{C}$ ) generated by $H^{1}(X)$. 
Restricting the fiber functor $\omega$ to $\mathcal{C}_{X}$ we get an equivalence between the category $\mathcal{C}_{X}$ and the category of graded $\mathcal{A}_{\bullet}\left(\mathcal{C}_{X}\right)$-comodules in $\mathcal{P}_{X}$. There is the corresponding Lie coalgebra

$$
\mathcal{L}_{\bullet}\left(\mathcal{C}_{X}\right)=\frac{\mathcal{A}_{\bullet}\left(\mathcal{C}_{X}\right)}{\mathcal{A}_{>0}\left(\mathcal{C}_{X}\right)^{2}} \subset \mathcal{L}_{\bullet}(\mathcal{C})
$$

and the dual Lie algebra $\mathrm{L}_{\bullet}\left(\mathcal{C}_{X}\right)$, a quotient of $\mathrm{L}_{\bullet}(\mathcal{C})$.

The Hopf algebra $\mathcal{A}_{\bullet}(\mathcal{C})$ can be defined using the framed objects in $\mathcal{C}$, see G3.

The Lie coalgebra $\mathcal{L}_{\bullet}(\mathcal{C})$ is the direct sum of its isotypical components $\mathcal{L}_{M}(\mathcal{C})$ over the set of all isomorphism classes of simple objects $M$ in $\mathcal{C}$ :

$$
\mathcal{L}_{\bullet}(\mathcal{C})=\oplus_{M} \mathcal{L}_{M}(\mathcal{C}) \bigotimes M^{\vee}
$$

\section{$10.2 \mathcal{C}$-motivic fundamental algebras of curves}

Below we recall main features of the fundamental Lie/Hopf algebras of an irreducible smooth open curve in the motivic framework and in the realizations. The classical point of view is presented in Section 1.2 and Section 8 . The $l$-adic one is recalled in the end.

Let $S=\left\{s_{0}, \ldots, s_{n}\right\} \subset X(F)$, and $v_{0}$ is a tangent vector at $s_{0} \in S$ defined over $F$. One should have a Hopf algebra $\mathrm{A}^{\mathcal{C}}\left(X-S, v_{0}\right)$ in the category $\mathcal{C}$, the $\mathcal{C}$-motivic fundamental Hopf algebra of $X-S$ with the tangential base point $v_{0}$. It was defined in the setups ii) and iii) in [D1, and in the setup iv) in [DG]. For the setup v) see [HZ]. Set

$$
\mathbb{A}_{X, S^{*}}^{\mathcal{C}}:=\operatorname{gr}_{\bullet}^{W} \mathrm{~A}^{\mathcal{C}}\left(X-S, v_{0}\right) .
$$

We use notation $\mathrm{A}$ and $\mathbb{A}$ for $\mathrm{A}^{\mathcal{C}}\left(X-S, v_{0}\right)$ and $\mathbb{A}_{X, S^{*}}^{\mathcal{C}}$. The tangential base point $v_{0}$ gives rise to a canonical morphism, the "canonical loop around $s_{0}$ ":

$$
X_{s_{0}}: \mathbb{Q}(1) \longrightarrow \mathrm{A} .
$$

The punctures $s \in S^{*}$ provide conjugacy classes in A, corresponding to "loops around $s$ ". By passing to the associated graded we arrive at canonical morphisms

$$
X_{s}: \mathbb{Q}(1) \longrightarrow \mathbb{A}, \quad s \in S^{*} .
$$

A derivation $\mathcal{D}$ of the algebra $\mathbb{A}$ is called special if there exist objects $B_{s}$ in $\mathbb{A}$ such that

$$
\mathcal{D}\left(X_{s}\right)=\left[B_{s}, X_{s}\right], \quad s \in S^{*} ; \quad \text { and } \quad \mathcal{D}\left(X_{s_{0}}\right)=0 .
$$

The formula on the left means that $\mathcal{D}\left(X_{s}(\mathbb{Q}(1))\right)$ is isomorphic to the image of the commutator map applied to the object $B_{s} \otimes X_{s}(\mathbb{Q}(1))$. Special derivations form a Lie algebra, denoted by $\operatorname{Der}^{S} \mathbb{A}$.

Set

$$
\mathbb{S}^{*}=\mathbb{Q}\left[S^{*}\right] \otimes H_{2}(X), \quad \mathbb{H}=H_{1}(X) ; \quad \mathbb{V}:=\mathbb{S}^{*} \oplus \mathbb{H} \cong \mathrm{gr}^{W} H_{1}(X-S) .
$$

Notice that here $H_{2}(X)=\mathbb{Q}(1)$ tells the weight grading. In the linear algebra considerations before the additional data given by the weight grading was not taking into account.

Let $T_{\mathbb{V}}$ be the tensor algebra of $\mathbb{V}$.

Lemma 10.2. The Hopf algebra $\mathbb{A}$ is isomorphic to the tensor algebra $\mathrm{T}_{\mathbb{V}}$.

Proof. The Hopf algebra (18) is identified with the Betti realization of the motivic fundamental Hopf algebra of $X-S$. This implies Lemma 10.2. 
An example: $l$-adic fundamental algebras. Let $\pi_{1}^{(l)}:=\pi_{1}^{(l)}\left(X-S, v_{0}\right)$ be the pro- $l$ completion of the fundamental group of $X-S$ with the tangential base point at $v_{0}$. It gives rise to a pronilpotent Lie algebra $\mathrm{L}^{(l)}\left(X-S, v_{0}\right)$ over $\mathbb{Q}_{l}$ as follows. Let $\pi_{1}^{(l)}(k)$ be the lower central series of $\pi_{1}^{(l)}$. Then $\pi_{1}^{(l)} / \pi_{1}^{(l)}(k)$ is an $l$-adic Lie group, and

$$
\mathrm{L}^{(l)}\left(X-S, v_{0}\right):=\lim _{\longleftarrow} \operatorname{Lie}\left(\pi_{1}^{(l)} / \pi_{1}^{(l)}(k)\right) .
$$

It is the $l$-adic realization of the fundamental Lie algebra $\mathrm{L}\left(X-S, v_{0}\right)$.

If $X$ is defined over $\mathbb{C}$, the comparison theorem between the Betti and $l$-adic realizations gives rise to a Lie algebra isomorphism $\mathrm{L}^{(l)}\left(X-S, v_{0}\right)=\pi_{1}^{\text {nil }}\left(X(\mathbb{C})-S, v_{0}\right) \otimes \mathbb{Q}_{l}$.

If $X$ is defined over a field $F$, the Galois group $\operatorname{Gal}(\bar{F} / F)$ acts by automorphisms of $\mathrm{L}^{(l)}(X-$ $\left.S, v_{0}\right)$, giving rise to a canonical homomorphism

$$
\operatorname{Gal}(\bar{F} / F) \longrightarrow \operatorname{AutL}^{(l)}\left(X-S, v_{0}\right) .
$$

The tangent vector $v_{0}$ provides a morphism of Galois modules, the "canonical loop around $s_{0}$ ":

$$
X_{s_{0}}: \mathbb{Q}_{l}(1) \longrightarrow \mathrm{L}^{(l)}\left(X-S, v_{0}\right) .
$$

Thus every puncture $s \in S^{*}$ gives rise to a conjugacy class in $\mathrm{L}^{(l)}\left(X-S, v_{0}\right)$ preserved by the Galois group. An automorphism of $\mathrm{L}^{(l)}\left(X-S, v_{0}\right)$ is special if it preserves $X_{s_{0}}\left(\mathbb{Q}_{l}(1)\right)$ and the conjugacy classes around all other punctures. Denote by $\operatorname{Aut}^{S} \mathrm{~L}^{(l)}\left(X-S, v_{0}\right)$ the group of all special automorphisms of $\mathrm{L}^{(l)}\left(X-S, v_{0}\right)$. We get a map

$$
\operatorname{Gal}\left(\bar{F} / F\left(\zeta_{l \infty}\right)\right) \longrightarrow \operatorname{Aut}^{S} \mathrm{~L}^{(l)}\left(X-S, v_{0}\right) .
$$

\subsection{The Lie algebra of special derivations in the motivic set-up}

We use a shorthand

$$
\mathcal{C}_{\mathbb{V}}:=\frac{\mathrm{T}_{\mathbb{V}}}{\left[\mathrm{T}_{\mathbb{V}}, \mathrm{T}_{\mathbb{V}}\right]}, \quad \mathcal{H}_{X}=\mathcal{H}:=H^{2}(X)
$$

We are going to define an action of $\mathcal{C}_{\mathbb{V}} \otimes \mathcal{H}$ by special derivations of the algebra $\mathrm{T}_{\mathbb{V}}$, and introduce a Lie algebra structure on $\mathcal{C}_{\mathbb{V}} \otimes \mathcal{H}$, making this action into an action of a Lie algebra.

Since $T_{\mathbb{V}}$ is the free associative algebra in a semi-simple category generated by $\mathbb{V}$, there is a non-commutative differential map

$$
\mathbb{D}: \mathcal{C}_{\mathbb{V}} \longrightarrow \mathrm{T}_{\mathbb{V}} \otimes \mathbb{V}, \quad \mathbb{D} \mathcal{C}\left(a_{0} \otimes a_{1} \otimes \ldots \otimes a_{m}\right):=\mathrm{Cyc}_{m+1}\left(a_{0} \otimes \ldots \otimes a_{m-1}\right) \otimes a_{m} .
$$

Here $\mathrm{Cyc}_{m+1}$ means the cyclic sum. So on the the right there is a sum of $m+1$ summands obtained by the cyclic shifts of $\left(a_{0} \otimes \ldots \otimes a_{m-1}\right) \otimes a_{m}$.

Let us define a canonical map

$$
\langle * \cap \mathcal{H} \cap *\rangle: \mathbb{V} \otimes \mathcal{H} \otimes \mathbb{V} \longrightarrow \mathbb{Q} \oplus \mathbb{S}^{*} .
$$

Its only non-zero components are the following:

$$
\langle * \cap \mathcal{H} \cap *\rangle_{\mathbb{H}}: \mathbb{H} \otimes \mathcal{H} \otimes \mathbb{H} \longrightarrow \mathbb{Q}, \quad\langle * \cap \mathcal{H} \cap *\rangle_{\mathbb{S}^{*}}: \mathbb{S}^{*} \otimes \mathcal{H} \otimes \mathbb{S}^{*} \longrightarrow \mathbb{S}^{*} .
$$

The first one is the intersection pairing on $H_{1}(X)$. The second is given by $\left\langle X_{s} \cap \mathcal{H} \cap X_{t}\right\rangle_{\mathbb{S}^{*}}=\delta_{s t} X_{s}$. 
An action of $\mathcal{C}_{\mathbb{V}} \otimes \mathcal{H}$ by special derivations of $\mathrm{T}_{\mathbb{V}}$. We are going to define a map

$$
\kappa: \mathcal{C}_{\mathbb{V}} \otimes \mathcal{H} \longrightarrow \operatorname{Der}^{S}\left(\mathrm{~T}_{\mathbb{V}}\right)
$$

Since the algebra $T_{\mathbb{V}}$ is free, to define its derivation we just define it on the generators, i.e. produce an element in $T_{\mathbb{V}} \otimes \mathbb{V}^{\vee}$. We get it as a composition

$$
\mathcal{C}_{\mathbb{V}} \otimes \mathcal{H} \stackrel{\mathbb{D} \otimes I d}{\longrightarrow} \mathrm{T}_{\mathbb{V}} \otimes\left(\mathbb{H} \oplus \mathbb{S}^{*}\right) \otimes \mathcal{H} \stackrel{\stackrel{(228)}{\longrightarrow}}{\longrightarrow} \mathrm{T}_{\mathbb{V}} \otimes\left(\mathbb{H}^{\vee} \oplus \mathbb{S}^{*} \otimes \mathbb{S}^{* \vee}\right) \cong \mathrm{T}_{\mathbb{V}} \otimes \mathbb{H}^{\vee} \bigoplus \mathbb{S}^{*} \otimes \mathrm{T}_{\mathbb{V}} \otimes \mathbb{S}^{* \vee}
$$

followed by the commutator map $\mathbb{S}^{*} \otimes \mathrm{T}_{\mathbb{V}} \longrightarrow \mathrm{T}_{\mathbb{V}}$. The map $\mathrm{T}_{\mathbb{V}} \otimes \mathbb{S}^{*} \otimes \mathcal{H} \longrightarrow \mathrm{T}_{\mathbb{V}} \otimes \mathbb{S}^{*} \otimes \mathbb{S}^{*}{ }^{*}$ in the second arrow is obtained using the map $\mathbb{S}^{*} \otimes \mathcal{H} \otimes \mathbb{S}^{*} \longrightarrow \mathbb{S}^{*}$ from (228).

A Lie bracket on $\mathcal{C}_{\mathbb{V}} \otimes \mathcal{H}$. There is a canonical map $\theta: \mathcal{C}_{\mathbb{V}} \otimes \mathcal{H} \otimes \mathcal{C}_{\mathbb{V}} \longrightarrow \mathrm{T}_{\mathbb{V}}$ given as $\mathcal{C}_{\mathbb{V}} \otimes \mathcal{H} \otimes \mathcal{C}_{\mathbb{V}} \stackrel{\mathbb{D} \otimes \mathrm{Id} \otimes \mathbb{D}}{\longrightarrow} \mathrm{T}_{\mathbb{V}} \otimes \mathbb{V} \otimes \mathcal{H} \otimes \mathrm{T}_{\mathbb{V}} \otimes \mathbb{V} \longrightarrow \mathrm{T}_{\mathbb{V}} \otimes \mathrm{T}_{\mathbb{V}} \otimes \mathbb{V} \otimes \mathcal{H} \otimes \mathbb{V} \stackrel{\text { 227] }}{\longrightarrow} \mathrm{T}_{\mathbb{V}} \otimes \mathrm{T}_{\mathbb{V}} \otimes\left(\mathbb{Q} \oplus \mathbb{S}^{*}\right) \longrightarrow \mathrm{T}_{\mathbb{V}}$

The last map is the product for the $\mathbb{Q}$-component, and the commutator map [,] : $\mathrm{T}_{\mathbb{V}} \otimes \mathrm{T}_{\mathbb{V}} \rightarrow \mathrm{T}_{\mathbb{V}}$ followed by the product with $\mathbb{S}^{*}$ for the second component. We define a Lie bracket on $\mathcal{C}_{\mathbb{V}} \otimes \mathcal{H}$ as follows:

$$
\{F \otimes \mathcal{H}, G \otimes \mathcal{H}\}:=\mathcal{C} \circ \theta(F \otimes \mathcal{H} \otimes G-G \otimes \mathcal{H} \otimes F) \otimes \mathcal{H}
$$

Explicit formulas. Let $\mathbb{H}=\oplus_{i} p_{i}$ be a decomposition into simple objects. Then, given an $F \in \mathcal{C}_{\mathbb{V}}$, we have

$$
\mathbb{D} F=\sum_{i} \frac{\partial F}{\partial p_{i}} \otimes p_{i}+\sum_{s \in S^{*}} \frac{\partial F}{\partial X_{s}} \otimes X_{s} .
$$

Now the action of the derivation $\kappa_{F \otimes \mathcal{H}}$ obtained by applying the map $\kappa$ to $F \otimes \mathcal{H}$ is given by

$$
\begin{aligned}
& \kappa_{F \otimes \mathcal{H}}: p \longmapsto \frac{\partial F}{\partial q}\langle q \cap \mathcal{H} \cap p\rangle, \quad X_{s_{0}} \longmapsto 0, \quad X_{s} \longmapsto\left[X_{s}, \frac{\partial F}{\partial X_{s}}\right], \quad s \in S^{*} . \\
& \theta: F \otimes \mathcal{H} \otimes G \longmapsto \sum_{i, j} \frac{\partial F}{\partial p_{i}} \otimes \frac{\partial G}{\partial p_{j}} \otimes\left\langle p_{i} \cap \mathcal{H} \cap p_{j}\right\rangle+\sum_{s \in S^{*}}\left[\frac{\partial F}{\partial X_{s}}, \frac{\partial G}{\partial X_{s}}\right] \otimes\left\langle X_{s} \cap \mathcal{H} \cap X_{s}\right\rangle . \\
& \{F \otimes \mathcal{H}, G \otimes \mathcal{H}\}=2 \sum_{s \in S^{*}} \mathcal{C}\left(\left[\frac{\partial F}{\partial X_{s}}, \frac{\partial G}{\partial X_{s}}\right] \otimes X_{s}\right) \otimes \mathcal{H}+ \\
& \sum_{p, q}\langle p \cap \mathcal{H} \cap q\rangle \otimes \mathcal{C}\left(\frac{\partial F}{\partial p} \otimes \frac{\partial G}{\partial q}-\frac{\partial G}{\partial p} \otimes \frac{\partial F}{\partial q}\right) \otimes \mathcal{H}
\end{aligned}
$$

Just like in Section 8, we define $\mathcal{C}_{\mathbb{V}}^{\prime}$ as the quotient of $\mathcal{C}_{\mathbb{V}}$ by $\oplus_{s \in S^{*}} \mathbb{Q}\left[X_{s}\right]$.

Theorem 10.3. The map $\kappa$ is a morphism of Lie algebras. It induces an isomorphism

$$
\kappa^{\prime}: \mathcal{C}_{\mathbb{V}}^{\prime} \otimes \mathcal{H} \stackrel{\sim}{\longrightarrow} \operatorname{Der}^{S}\left(\mathrm{~T}_{\mathbb{V}}\right) .
$$

The map $\kappa$ provides an isomorphism of Lie algebras

$$
\kappa: \mathcal{C} \mathcal{L} i e_{\mathbb{V}} \otimes \mathcal{H} \stackrel{\sim}{\longrightarrow} \operatorname{Der}^{S}\left(\mathrm{~L}_{\mathbb{V}}\right) .
$$



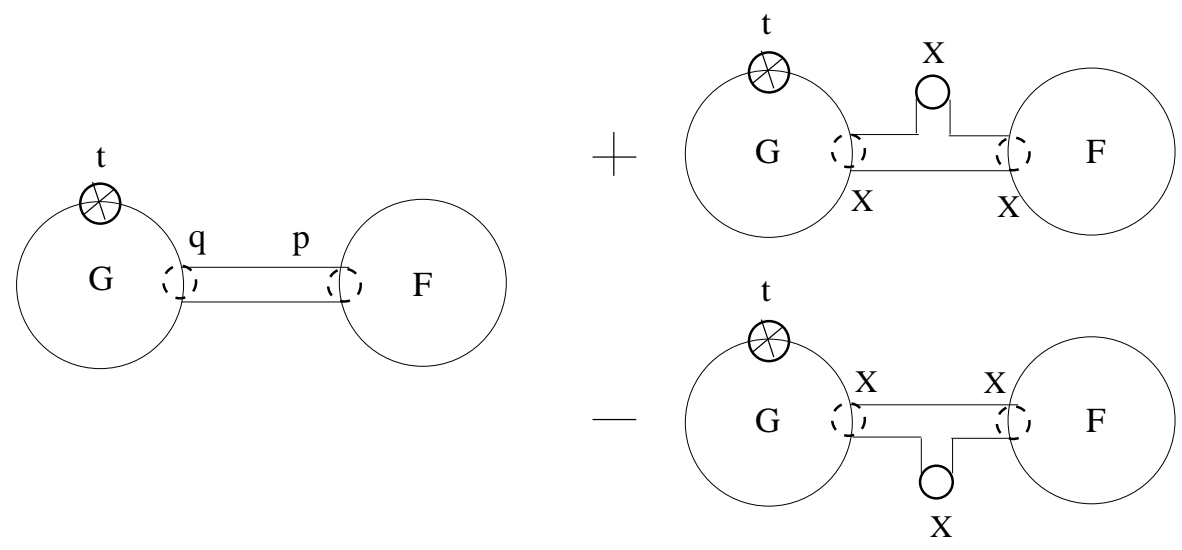

Figure 24: Calculation of $\kappa_{F \otimes \mathcal{H}} \circ \kappa_{G \otimes \mathcal{H}}(h)$.

Proof. Let us show that the map (232) respects the brackets. Let us calculate how the commutator $\left[\kappa_{F \otimes \mathcal{H}}, \kappa_{G \otimes \mathcal{H}}\right]$ acts on $h \subset \mathbb{H}$. To calculate the $\mathbb{H}$-part of the action, we have to insert $\partial F / \partial p$ instead of each factor $q$ in $\partial G / \partial t$ - this operation is denoted by $\stackrel{q}{\rightarrow}$. We get:

$$
\begin{gathered}
\kappa_{F \otimes \mathcal{H}} \circ \kappa_{G \otimes \mathcal{H}}: h \longmapsto \sum_{t} \kappa_{F \otimes \mathcal{H}}\left(\frac{\partial G}{\partial t}\right) \otimes\langle t \cap \mathcal{H} \cap h\rangle= \\
\sum_{t}\left(\sum_{p, q} \frac{\partial F}{\partial p} \stackrel{q}{\rightarrow} \frac{\partial G}{\partial t} \otimes\langle p \cap \mathcal{H} \cap q\rangle+\sum_{s \in S^{*}}\left[\frac{\partial F}{\partial X_{s}}, \frac{\partial G}{\partial X_{s}}\right] X_{s}\right) \otimes\langle t \cap \mathcal{H} \cap h\rangle .
\end{gathered}
$$

The (left) sums are over bases $\{t\},\{p\},\{q\}$ in $\mathbb{H}$. The result is visualized on Fig 24. Big circles show the cyclic words $F$ and $G$; little circles show the variables added, and the punctured little circles show the variables removed in the process of calculation. The added / removed factors shown nearby. The bridge between big circles shows how we make the product. The special role of $t$ is emphasized by a cross. It is clear from this that $\left[\kappa_{F \otimes \mathcal{H}}, \kappa_{G \otimes \mathcal{H}}\right]$ acts on $h$ just like the commutator $\kappa_{\{F \otimes \mathcal{H}, G \otimes \mathcal{H}\}}$. The action on $X_{s}$ is treated similarly - the only difference is that we have to replace the operation of removing of $t$ by the commutator with $X_{s}$.

The rest follows from this and Proposition 8.4 by going to the Betti realization, or repeating the argument in the motivic set-up.

\subsection{Motivic correlators on curves}

We define three versions of the motivic correlators on curves, which differ in our treatment of the base points: base point motivic correlators, symmetric motivic correlators, and averaged base point motivic correlators.

1. Base point motivic correlators on curves. Similar to (225), set

$$
\mathbb{L}_{X, S^{*}}:=\operatorname{gr}_{\bullet}^{W} \mathrm{~L}^{\mathcal{C}}\left(X-S, v_{0}\right) .
$$

Since the fundamental Lie algebra $\mathrm{L}^{\mathcal{C}}\left(X-S, v_{0}\right)$ of the curve is a pro-object in the category $\mathcal{C}_{X}$, the motivic Galois Lie algebra $\mathrm{L}_{\bullet}\left(\mathcal{C}_{X}\right)$ acts by the special derivations on its associate graded for 
the weight filtration (234), and so we get a map

$$
\mathrm{L} \bullet\left(\mathcal{C}_{X}\right) \longrightarrow \operatorname{Der}^{S} \mathbb{L}_{X, S^{*}}
$$

Identifying Lie algebras $\operatorname{Der}^{S} \mathbb{L}_{X, S^{*}}$ and $\mathcal{C} \mathcal{L} i e_{\mathbb{V}} \otimes H^{2}(X)$ via isomorphism (233), and dualizing map (235), we arrive at a Lie coalgebra map

$$
\Psi_{v_{0}}: \mathcal{C} \mathcal{L} i e_{X, S^{*}}^{\vee} \otimes H_{2}(X) \longrightarrow \mathcal{L} \bullet\left(\mathcal{C}_{X}\right)
$$

Recall notations (226). Abusing notation, write $X_{s}^{\vee}$ for $X_{s}(\mathbb{Q}(1))^{\vee}$. There is an isomorphism

$$
\Phi: \bigoplus_{m=1}^{\infty} \bigoplus_{a_{i} \in S^{*}}\left(\mathbb{A}_{\mathbb{H} \vee} \otimes X_{a_{0}}^{\vee}\right) \otimes\left(\mathbb{A}_{\mathbb{H} \vee} \otimes X_{a_{1}}^{\vee}\right) \otimes \ldots \otimes\left(\mathbb{A}_{\mathbb{H} \vee} \otimes X_{a_{m}}^{\vee}\right) \otimes \mathbb{A}_{\mathbb{H}^{\vee}} \stackrel{\sim}{\longrightarrow} \mathbb{A}_{\mathbb{V} \vee}
$$

Recall that $\mathcal{C} \mathcal{L} i e_{X, S^{*}}^{\vee}$ is the quotient of the cyclic envelope of $A_{\mathbb{V} \vee}$ by the shuffle relations:

$$
\mathcal{C} \mathcal{L} i e_{X, S^{*}}^{\vee}:=\frac{\mathcal{C}\left(\mathrm{A}_{\mathbb{V} \vee}\right)}{\text { Shuffle relations }}
$$

We denote by $\Phi_{\text {cyc }}$ the map $\Phi$ followed by the projection to the cyclic envelope of $\mathbb{A}_{\mathbb{V} \vee}$. Projecting then to the quotient (238) by the shuffle relations, and applying the map $\Psi_{v_{0}}$, see (236), we arrive at an element of the motivic Lie coalgebra $\mathcal{L}_{\bullet}\left(\mathcal{C}_{X}\right)$. This is our first definition:

Definition 10.4. Given points $a_{i} \in S^{*}$ and simple summands $M_{0}, \ldots, M_{m}$ of $\mathbb{A}_{\mathbb{H}} \vee$, we set $w=$ $2 m+\sum \mathrm{wt}\left(M_{i}\right)$, and define the base point motivic correlator

$$
\operatorname{Cor}_{X ; v_{0}}\left(\left\{a_{0}\right\} \otimes M_{0} \otimes\left\{a_{1}\right\} \otimes M_{1} \otimes \ldots \otimes\left\{a_{m}\right\} \otimes M_{m}\right)(1) \subset \mathcal{L}_{w}\left(\mathcal{C}_{X}\right)
$$

as the image of $\mathcal{C}\left(X_{a_{0}}^{\vee} \otimes M_{0} \otimes \ldots \otimes X_{a_{m}}^{\vee} \otimes M_{m}\right)(1)$ under the map $\Psi_{v_{0}} \circ \Phi_{\text {cyc }} 9$

Lemma 10.5. The object (239) lies in the $M_{0}^{\vee} \otimes \ldots \otimes M_{m}^{\vee}(m)$-isotypical component of $\mathcal{L}_{\bullet}\left(\mathcal{C}_{X}\right)$.

Proof. Recall decomposition (224). The object on the left in (239) is $M_{0} \otimes \ldots \otimes M_{m}(-m)$. The isotypical component of $\mathcal{L}_{\bullet}\left(\mathcal{C}_{X}\right)$ is labeled by its dual.

Using description of the Hopf algebra $\mathcal{A}_{\bullet}\left(\mathcal{C}_{X}\right)$ via minimal framed objects in $\mathcal{C}_{X}($ G3 $]$ ), and a similar description of the Lie coalgebra $\mathcal{L}_{\bullet}\left(\mathcal{C}_{X}\right)$, we conclude that the object (239) provides a minimal framed object in $\mathcal{C}_{X}$, well-defined modulo products of non-trivial objects in $\mathcal{C}_{X}$.

2. Symmetric motivic correlators on curves. Let us employ the symmetric description of the Lie algebra of special derivations (Section 8.3) in the motivic set-up. There is a Lie algebra $\overline{\mathcal{C}}_{X, S}$ and its Lie subalgebra $\overline{\mathcal{C L}} \boldsymbol{L} e_{X, S}$ in the $\mathcal{C}$-motivic category, whose Betti realizations are the Lie algebras $\mathcal{C} \mathcal{L} i e\left(\overline{\mathrm{A}}_{X, S}\right)$ and $\mathcal{C} \mathcal{L} i e\left(\overline{\mathrm{L}}_{X, S}\right)$. Denote by $\overline{\mathcal{C}}_{X, S}^{\vee}$ and $\overline{\mathcal{C L}} i e_{X, S}^{\vee}$ the dual Lie coalgebras.

There is a Lie coalgebra map

$$
\Psi^{\mathrm{sym}}: \overline{\mathcal{C}} \mathcal{L} i e_{X, S}^{\vee} \otimes H^{2}(X) \longrightarrow \mathcal{L} \bullet\left(\mathcal{C}_{X}\right) .
$$

\footnotetext{
${ }^{9}$ The twist by $\mathbb{Q}(1)$ comes from the factor $H_{2}(X)$ in (236).
} 
Definition 10.6. A symmetric motivic correlator is the image of $W \in \overline{\mathcal{C L}}^{\vee}{ }_{X, S}(1)$ under map (240):

$$
\operatorname{Cor}_{X}^{\mathrm{sym}}(W):=\Psi^{\mathrm{sym}}(W) \in \mathcal{L}\left(\mathcal{C}_{X}\right) .
$$

The Lie coalgebras in question are related as follows:

$$
\overline{\mathcal{C}} \mathcal{L} i e_{X, S}^{\vee} \longleftarrow \overline{\mathcal{C}}_{X, S}^{\vee} \hookrightarrow \mathcal{C}_{X, S}^{\vee}
$$

The left map is an epimorphism. In general there seems to be no simple description of the subspace $\overline{\mathcal{C}}_{X, S}^{\vee}$ in $\mathcal{C}_{X, S}^{\vee}$. However when $X$ is either a rational or an elliptic curve, such a description exists.

Lemma 10.7. Let $X$ be the projective line $\mathbb{P}^{1}$. Then an element

$$
\sum_{a_{0}, \ldots, a_{m} \in S} c_{a_{0}, \ldots, a_{m}} \mathcal{C}\left(X_{a_{0}}^{\vee} \otimes \ldots \otimes X_{a_{m}}^{\vee}\right) \in \mathcal{C}_{\mathbb{P}^{1}, S}^{\vee}
$$

lies in the subspace $\overline{\mathcal{C}}_{\mathbb{P}^{1}, S}^{\vee}$ if and only if $\sum_{s \in S} c_{a_{0}, \ldots, a_{k-1}, s, a_{k+1}, \ldots, a_{m}}=0$ for any $k=0, \ldots, m$.

Proof. Elements (241) for arbitrary coefficients $c_{a_{0}, \ldots, a_{m}} \in \mathbb{Q}$ give a basis in $\mathcal{C}_{\mathbb{P}^{1}, S}^{\vee}$. The orthogonality to the two-sided ideal generated by $\sum_{s \in S} X_{s}$ is precisely the condition on the coefficients.

Lemma 10.8. Let $E$ be an elliptic curve, $\mathbb{H}^{\vee}=H^{1}(E)$. Then the subspace $\overline{\mathcal{C}}_{E, S}^{\vee}$ consists of elements

$$
\sum_{a_{i} \in S} c_{a_{0}, n_{0} ; \ldots, a_{m}, n_{m}} \mathcal{C}\left(X_{a_{0}}^{\vee} \otimes S^{n_{0}} \mathbb{H}^{\vee} \otimes \ldots \otimes X_{a_{m}}^{\vee} \otimes S^{n_{m}} \mathbb{H}^{\vee}\right) \in \mathcal{C}_{E, S}^{\vee}
$$

such that for any $k=0, \ldots, m$ one has $\sum_{a_{k} \in S} c_{a_{0}, n_{0} ; \ldots, a_{m}, n_{m}}=0$.

Proof. We spell the argument in the Betti realization. So $\mathbb{H}^{\vee}$ has a symplectic basis $p, q$. A basis of $\mathcal{C}_{E, S}^{\vee}$ is given by cyclic words $\mathcal{C}\left(a_{0} \otimes F_{0}(p, q) \otimes \ldots \otimes a_{m} \otimes F_{m}(p, q)\right)$, where $a_{i} \in S$ and $F_{i}(p, q)$ are non-commutative polynomials in $p, q$. The defining ideal is generated by the element $[p, q]+\sum_{a \in S} X_{a}$. So expressing $[p, q]$ as $-\sum_{a \in S} X_{a}$ we can replace the polynomials $F_{i}(p, q)$ by commutative polynomials $G_{i}(p, q)$. We may assume that $G_{i}$ are homogeneous of degree $n_{i}$. Since $G_{i}(p, q)$ are commutative polynomials, they are orthogonal to $[p, q]$. So the orthogonality to the element $[p, q]+\sum_{a \in S} X_{a}$ boils down to the orthogonality to $\sum_{a \in S} X_{a}$.

Remark. The symmetric motivic correlator map does not depend on the choice of the base point. It has the same image in the motivic Lie coalgebra as the one defined by using a base point. This apparent "contradiction" is resolved as follows. A choice of the base point provides a way to choose elements in the image of the motivic correlator map, without changing the latter. Different base points provide different representations, built using different supplies of elements in the same space. For example, as we see in Section 10.5.1, the elements of the Jacobian $J_{X} \otimes \mathbb{Q}$ of $X$ provided by the symmetric motivic correlators are $s_{i}-s_{j}$. The ones provided by the motivic correlators corresponding to a tangential base point at $s_{0}$ are $s_{i}-s_{0}$. These are two different collection of elements of $J_{X} \otimes \mathbb{Q}$; however they span the same subspace.

Similarly the symmetric Hodge correlators are complex numbers defined by the curve $X-S$. The $\mathbb{Q}$-vector space spanned by them does not depend on the choice of the base point, or a Green function. However choosing different base points we get different real MHS's - the latter depend not only on the $\mathbb{Q}$-vector subspace spanned by the periods, but also on the way we view its elements as the matrix coefficients. 
3. Averaged base point motivic correlators. Let us choose for every point $s \in S$ a tangent vector $v_{s}$ at $s$. There are natural choices for the elliptic and modular curves.

Recall the canonical morphism of Lie coalgebras assigned to a tangential base point $v_{s}$ at $s$ :

$$
\operatorname{Cor}_{X, S-\{s\} ; v_{s}}: \mathcal{C} \mathrm{T}\left(\mathbb{H}_{X, S-\{s\}}^{\vee}\right) \otimes H_{2}(X) \longrightarrow \mathcal{L}_{\mathrm{Mot}}
$$

The collection of maps (243), for different choices of $s \in S$, is organized into a Lie coalgebra map

$$
\oplus_{s \in S} \mathcal{C} \mathrm{T}\left(\mathbb{H}_{X, S-\{s\}}^{\vee}\right) \otimes H_{2}(X) \longrightarrow \mathcal{L}_{\mathrm{Mot}}
$$

Here on the left stands a direct sum of the Lie coalgebras. It is handy extend $S-\{s\}$ to $S$, and enlarge the Lie coalgebra on the left to a Lie coalgebra

$$
\mathcal{C} T\left(\mathbb{H}_{X, S}^{\vee}\right) \otimes H_{2}(X) \otimes \mathbb{Q}[S]
$$

where the added elements have the coproduct zero. So the Lie coalgebra on the left of (244) is the quotient of (245). The composition (245) $\rightarrow(244)$ provides a Lie coalgebra map

$$
\operatorname{Cor}_{X-S}^{\mathrm{av}}: \mathcal{C} \mathrm{T}\left(\mathbb{H}_{X, S}^{\vee}\right) \otimes H_{2}(X) \otimes \mathbb{Q}[S] \longrightarrow \mathcal{L}_{\mathrm{Mot}},
$$

so that map (243) is the component of map (246) assigned to the factor $\{s\} \in \mathbb{Q}[S]$.

The averaged base point motivic correlator map arises when we take $\frac{1}{|S|} \sum_{s \in S}\{s\} \in \mathbb{Q}[S]$. It allows to work in towers of curves, see Section 12.2

\subsection{Examples of motivic correlators}

1. The Jacobian of $X$. Let $J_{X}(F)$ be the Jacobian of the curve $X$. It is well known that in the motivic set-up (i) one should have

$$
\operatorname{Ext}_{\text {Mot }}^{1}(\mathbb{Q}(0), \mathbb{H})=\mathcal{L}_{1}\left(\mathcal{C}_{X}\right)=J_{X}(F) \otimes \mathbb{Q} .
$$

The motivic correlator $\operatorname{Cor}_{X, s_{0}}\left(\{a\} \otimes \mathbb{H}^{\vee}\right)$, see Fig. 25, is given by the point $\{a\}-\left\{s_{0}\right\}$ in the Jacobian $J_{X}(F)$. Notice the importance of the base point $s_{0}$. In the symmetric description the correlators are

$$
\operatorname{Cor}_{X}^{\mathrm{sym}} \mathcal{C}\left((\{a\}-\{b\}) \otimes \mathbb{H}^{\vee}\right)=a-b \in J_{X} \otimes \mathbb{Q} .
$$

They are defined only on degree zero divisors. In any case we get the same supply of elements of the motivic Lie coalgebra. In the $l$-adic set-up (iii) the target group is $J_{X}(F) \otimes \mathbb{Q}_{l}$.

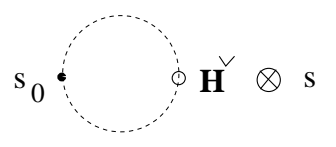

Figure 25: The motivic correlator for $\mathcal{C}\left(\left\{s_{0}\right\} \otimes \mathbb{H}^{\vee}\right)$ is the point $s-s_{0}$ of the Jacobian of $X$. 
2. The rational curve case. Let $X=\mathbb{P}^{1}$, the set $S$ includes $\{0\} \cup\{\infty\}$, and $v=\partial / \partial t$ is the canonical vector at 0 . Denote by $d t / t$ the cohomology class given by the form $d t / t$ in the De Rham realization. The motivic correlators in this case are

$$
\mathbb{L}_{n_{0}, \ldots, n_{m}}^{\mathcal{M}}\left(a_{0} ; \ldots ; a_{m}\right):=\operatorname{Cor}_{\mathbb{P}^{1} ; v}\left(\left\{a_{0}\right\} \otimes\{0\}^{\otimes n_{0}} \otimes \ldots \otimes\left\{a_{m}\right\} \otimes\{0\}^{\otimes n_{m}}\right) \subset \mathcal{L}_{w}\left(\mathcal{C}_{T}\right) .
$$

Here $a_{i} \in S^{*}, w=2\left(n_{0}+\ldots+n_{m}+m\right)$ is the weight, and $m$ is the depth (Section 9.5.9). We call them the cyclic motivic multiple polylogarithms. Their periods coincide with the corresponding multiple polylogarithms up to the lower depth terms. In Section 11,1.4 we derive an integral formula expressing their real periods as Hodge correlator type integrals of the classical polylogarithms.

Remark. In [G4], given an abelian group $G$, we defined dihedral Lie coalgebras, which are generated, as vector spaces, by symbols $\mathrm{L}_{n_{0}, \ldots, n_{m}}\left(g_{0} ; \ldots ; g_{m}\right), g_{i} \in G$. When $G=\mathbb{C}^{*}$, we related them to multiple polylogarithms, although to achieve this we had to work modulo the depth filtration. It is now clear that those generators match the motivic elements (247), and so their periods are the corresponding Hodge correlators rather then the single valued versions of the multiple polylogarithms. See Section 11 for more information.

An especially interesting case is when $S=\mu_{N} \cup\{0\} \cup\{\infty\}$. In this case we call the elements (247) the depth $m$ motivic multiple Dirichlet $L$-values. For instance when $S=\{0\} \cup\{1\} \cup\{\infty\}$, we get the depth $m$ motivic multiple $\zeta$-values.

3. The elliptic curves case. Let $X=E$ be an elliptic curve over $F$. If $E$ is not CM, the objects $S^{k} \mathbb{H}$ are simple. The symmetric motivic multiple elliptic polylogarithms are defined as

$$
\operatorname{Cor}_{E ; n_{0}, \ldots, n_{m}}^{\mathrm{sym}}\left(D_{0} ; \ldots ; D_{m}\right):=\operatorname{Cor}_{E}\left(D_{0} \otimes \mathrm{S}^{n_{0}} \mathbb{H}^{\vee} \otimes \ldots \otimes D_{m} \otimes \mathrm{S}^{n_{m}} \mathbb{H}^{\vee}\right)(1) \subset \mathcal{L}_{w}\left(\mathcal{C}_{E}\right)
$$

Here $D_{i}$ are degree zero divisors supported on $S$ and $w=n_{0}+\ldots+n_{m}+2 m$. When $E$ is a complex elliptic curve, their periods are given by the generalized Eisenstein-Kronecker series (Section 11,2).

4. Motivic correlators of torsion points on elliptic curves. Let $X=E$ be an elliptic curve, and $S=E[N]$ is the set of its $N$-torsion points. There is an almost canonical choice $v_{\Delta}$ of the tangent vector at 0 : its dual is given by 12 -th root of the section of $\Omega_{\mathcal{E} / \mathcal{M}}^{12}$, where $\mathcal{E}$ is the universal elliptic curve over the modular curve $\mathcal{M}$, given by the modular form $\Delta=q \prod\left(1-q^{n}\right)^{24}$. We employ the corresponding $E$-invariant vector field $v_{\Delta}$ on $E$, and assign to every missing point on $E-E[N]$ the tangent vector $\frac{1}{N} v_{\Delta}$. We arrive at the averaged base point motivic correlator map. It is obviously $E[N]$-invariant, and thus descends to a map of $E[N]$-coinvariants:

$$
\operatorname{Cor}_{E-E[N]}^{\mathrm{av}}:\left(\mathcal{C T}\left(\mathbb{H}_{E, E[N]}^{\vee}\right) \otimes \operatorname{Meas}(E[N])(1)\right)_{E[N]} \longrightarrow \mathcal{L}_{\mathrm{Mot}} .
$$

There is a unique $E[N]$-invariant volume 1 measure $\mu_{E}^{0}$ on $E[N]$. So we get a canonical map

$$
\mathrm{Cor}_{E-E[N]}^{0}: \mathcal{C} \mathrm{T}\left(\mathbb{H}_{E, E[N]}^{\vee}\right)(1)_{E[N]} \otimes \mu_{E}^{0} \longrightarrow \mathcal{L}_{\mathrm{Mot}} .
$$

These are the motivic correlators with the "averaged tangent vector" at the $N$-torsion points. 
5. Motivic double elliptic logarithms at torsion points. They were defined and studied in [G10]. Here is an alternative definition, coming from the action of the Galois group on the motivic fundamental group of an elliptic curve minus the torsion points. We use a shorthand

$$
\theta_{E}\left(a_{0}: \ldots: a_{n}\right):=\operatorname{Cor}_{E}^{0}\left(\left\{a_{0}\right\} \otimes \ldots \otimes\left\{a_{n}\right\}\right)(1), \quad a_{i} \in E_{\text {tors }} .
$$

It agrees with the notation in G10]. It is translation invariant.

Here is an $l$-adic / Hodge version of formula (76) expressing the Green function $G_{a}(x, y)$ via the Arakelov Green function. We spell it in the l-adic set-up.

Lemma 10.9. For any $x \in E(F)$, and any E-invariant vector field $v$ on $E$ one has

$$
\theta_{x}\left(a_{0}: a_{1}\right)=\theta_{E}\left(a_{0}: a_{1}\right)-\theta_{E}\left(x: a_{1}\right)-\theta_{E}\left(a_{0}: x\right)+C, \quad C \in \operatorname{Ext}_{\mathcal{C}}^{1}\left(\mathbb{Q}_{l}(0), \mathbb{Q}_{l}(2)\right) .
$$

Proof. Indeed,

$$
\delta \theta_{x}\left(a_{0}: a_{1}\right)=\operatorname{Cor}_{E, x}\left(\left\{a_{0}\right\} \otimes \mathbb{H}^{\vee}\right)(1) \wedge \operatorname{Cor}_{E, x}\left(\left\{a_{1}\right\} \otimes \mathbb{H}^{\vee}\right)(1)=\left(a_{0}-x\right) \wedge\left(a_{1}-x\right) \in \Lambda^{2} E(F) .
$$

The coproduct of the right hand side modulo $N$-torsion is the same. Therefore the difference lies in $\operatorname{Ext}_{\mathcal{C}}^{1}\left(\mathbb{Q}_{l}(0), \mathbb{Q}_{l}(2)\right)$. The latter $l$-adic Ext-group is rigid. Thus, as a variation in $x, a_{0}, a_{1}$, it is constant variation.

The constant $C$ for the canonical vector $v_{\Delta}$ is zero. Indeed, $v_{\Delta}$, viewed as a section over the modular curve, is defined over $\mathbb{Q}$, and we can take $E, x, a_{i}$ 's defined over $\mathbb{Q}$, and use the fact that $C$ is of geometric origin, and $K_{3}^{\text {ind }}(\mathbb{Q})=0$.

Lemma 10.10. Assume that $a_{i} \in E[N]$. Then, in the l-adic or Hodge set-ups, one has

$$
\delta \theta_{E}\left(a_{0}: a_{1}: a_{2}\right)=\operatorname{Cycle}_{\{0,1,2\}}\left(\theta_{E}\left(a_{0}: a_{1}\right) \wedge \theta_{E}\left(a_{1}: a_{2}\right)\right) \quad \text { modulo } N \text {-torsion. }
$$

Proof. We have $N \delta_{\text {Cas }} \theta_{E}\left(a_{0}: a_{1}: a_{2}\right)=0$. Indeed,

$$
N \delta_{\text {Cas }} \theta_{E}\left(a_{0}: a_{1}: a_{2}\right)=N \operatorname{Cycle}_{\{0,1,2\}}\left(\operatorname{Cor}_{E}^{0}\left(\left\{a_{0}\right\} \otimes \mathbb{H}^{\vee}\right)(1) \wedge \operatorname{Cor}_{E}^{0}\left(\mathbb{H}^{\vee} \otimes\left\{a_{1}\right\} \otimes\left\{a_{2}\right\}\right)(1)\right),
$$

and $N \operatorname{Cor}_{E}^{0}\left(\left\{a_{0}\right\} \otimes \mathbb{H}^{\vee}\right)=N a_{0}=0$. Using Lemma 10.9, $\delta_{S} \theta_{E}\left(a_{0}: a_{1}: a_{2}\right)$ equals

$$
\operatorname{Cycle}_{\{0,1,2\}} \frac{1}{N^{2}} \sum_{x \in E[N]} \theta_{x}\left(a_{0}: a_{1}\right) \wedge \theta_{x}\left(a_{0}: a_{2}\right)=\operatorname{Cycle}_{\{0,1,2\}}\left(\theta_{E}\left(a_{0}: a_{1}\right) \wedge \theta_{E}\left(a_{0}: a_{2}\right)\right) .
$$

The elements $\theta_{E}\left(a_{0}: a_{1}: a_{2}\right)$ from G10 have the same coproduct, and thus coincide with the ones above up to an element of $\operatorname{Ext}_{\mathcal{C}}^{1}\left(\mathbb{Q}_{l}(0), \mathbb{Q}_{l}(2)\right)$. Each of them is skew-symmetric in $a_{0}, a_{1}, a_{2}$. Since the difference between them is constant on the modular curve, this implies that it is zero. 
6. Motivic correlators of torsion points on a CM elliptic curve. Suppose that $E$ is a CM curve. Then it has a complex multiplication by an order in the ring of integers in an imaginary quadratic field $K$. Extending the scalars from $L$ to $L \otimes K$, we have a decomposition $\mathbb{H}^{\vee}=\psi \oplus \bar{\psi}$ into a sum of pure motives corresponding to the two Hecke Grössencharacters. Set $\psi^{n}:=\psi^{\otimes n}$ and $\bar{\psi}^{n}:=\bar{\psi}^{\otimes n}$. Then $\mathrm{S}^{n} \mathbb{H}^{\vee}=\oplus_{n^{\prime}+n^{\prime \prime}=n} \psi^{n^{\prime}} \bar{\psi}^{n^{\prime \prime}}$. So we decompose the motivic correlators accordingly.

Let $a_{i}$ be torsion points on $E$, and $\left(n_{i}^{\prime}, n_{i}^{\prime \prime}\right)$ non-negative integers. The corresponding motivic Hecke Grössencharacters multiple L-values $L_{E ; n_{0}^{\prime}, n_{0}^{\prime \prime} ; \ldots ; n_{m}^{\prime}, n_{m}^{\prime \prime}}\left(a_{0}: \ldots: a_{m}\right)$ are motivic correlators

$$
\operatorname{Cor}_{E}^{0}\left(\left\{a_{0}\right\} \otimes \psi^{n_{0}^{\prime}} \bar{\psi}_{0}^{n_{0}^{\prime \prime}} \otimes\left\{a_{1}\right\} \otimes \psi^{n_{1}^{\prime}} \bar{\psi}^{n_{1}^{\prime \prime}} \otimes \ldots \otimes\left\{a_{m}\right\} \otimes \psi^{n_{m}^{\prime}} \bar{\psi}_{m}^{n_{m}^{\prime \prime}}\right)(1) .
$$

7. The Fermat curves case. Let $\mathbb{F}_{N}$ be the projective Fermat curve $x^{N}+y^{N}=z^{N}$. Let $S$ be the intersection of $\mathbb{F}_{N}$ with the coordinate triangle. The group $\mu_{N}^{2}=\mu_{N}^{3} / \mu_{N}$ acts on the curve $\mathbb{F}_{N}$. The motive $H^{1}\left(\mathbb{F}_{N}\right) \otimes \mathbb{Z}\left[\zeta_{N}\right]$ is decomposed into a direct sum of rank one motives $\psi_{\chi}$ parametrized by the characters $\chi$ of $\mu_{N}^{2}$. These are Weil's Jacobi sums Grössencharacter motives.

We use the symmetric motivic correlators. Just like in the CM elliptic curve case, by using the motives $\psi_{\chi}$ and their powers, we get symmetric motivic correlators parametrised by cyclic tensor products of $\psi_{\chi}^{n}$ and the Tate motives assigned to the points of $S$.

8. The modular curves case. This is one of the most interesting cases, see Section 12

9. The depth filtration. Suppose that $s_{0} \in S^{\prime} \subset S \subset X$. Then the inclusion $X-S \subset X-S^{\prime}$ provides a projection of algebras $\mathrm{A}\left(X-S, v_{0}\right) \longrightarrow \mathrm{A}^{\mathcal{C}}\left(X-S^{\prime}, v_{0}\right)$. A depth filtration on the Hopf algebra $\mathrm{A}\left(X-S, v_{0}\right)$ is a filtration by powers of its kernel. One defines similarly a depth filtration on the Lie algebra $\mathrm{L}\left(X-S, v_{0}\right)$. It is induced by the embedding $\mathrm{L}\left(X-S, v_{0}\right) \hookrightarrow \mathrm{A}\left(X-S, v_{0}\right)$. Here are two examples.

(i) Assume that $X \neq \mathbb{P}^{1}$. The embedding $X-S \hookrightarrow X$ provides a canonical projection

$$
\mathrm{A}\left(X-S, v_{0}\right) \longrightarrow \mathrm{A}\left(X, s_{0}\right) .
$$

Let $\mathrm{I}_{X, S}$ be its kernel. A depth filtration $D$ on the Hopf algebra $\mathrm{A}\left(X-S, v_{0}\right)$ is the filtration $\mathrm{A}\left(X-S, v_{0}\right) \supset \mathrm{I}_{X, S} \supset \mathrm{I}_{X, S}^{2} \supset \ldots$ by powers of the ideal $\mathrm{I}_{X, S}$, indexed by $m=0,1,2, \ldots$. One has

$$
\mathrm{Gr}_{m}^{D} \mathrm{~A}\left(X-S, v_{0}\right)=\oplus_{s_{i} \in S^{*}} \mathrm{~A}_{X} \otimes X_{s_{0}} \otimes \mathrm{A}_{X} \otimes \ldots \otimes X_{s_{m}} \otimes \mathrm{A}_{X} .
$$

(ii) The depth filtration for $\mathbb{G}_{m}-S$ is defined using the embedding $\mathbb{G}_{m}-S \hookrightarrow \mathbb{G}_{m}$.

\section{Examples of Hodge correlators}

\subsection{Hodge correlators on $\mathbb{C P}^{1}-S$ and polylogarithms}

1. Multiple Green functions. Let $a_{0}, \ldots, a_{m}$ be points of a complex curve $X$. Let $\mu$ be a volume one measure on $X$. The Hodge correlator of a cyclic word $\mathcal{C}\left(\left\{a_{0}\right\} \otimes \ldots \otimes\left\{a_{m}\right\}\right)$ is the depth $m$ multiple Green function (Section 9 of [G1]):

$$
G_{\mu}\left(a_{0}, \ldots, a_{m}\right):=\sum_{\mathrm{T}} \operatorname{sgn}\left(E_{1} \wedge \ldots \wedge E_{2 m-1}\right) \int_{X^{m-1}} \omega_{2 m-2}\left(G_{E_{1}} \wedge \ldots \wedge G_{E_{2 m-1}}\right) .
$$


The sum is over all plane trivalent trees $T$ decorated by $a_{0}, \ldots, a_{m}$. The integral is over the product of copies of $X$ parametrised by the internal vertices of $T$. The function $G_{\mu}\left(a_{0}, \ldots, a_{m}\right)$ enjoys

- The dihedral symmetry: $G_{\mu}\left(a_{0}, \ldots, a_{m}\right)=G_{\mu}\left(a_{1}, \ldots, a_{m}, a_{0}\right)=(-1)^{m+1} G_{\mu}\left(a_{m}, \ldots, a_{0}\right)$.

- The shuffle relations: $\sum_{\sigma \in \Sigma_{p, q}} G_{\mu}\left(a_{0}, a_{\sigma(1)}, \ldots, a_{\sigma(m)}\right)=0$.

The first property is clear from the definition. The second follows from Proposition 3.5,

2. The Bloch-Wigner function. The simplest multiple Green function appears when $X=$ $\mathbb{P}^{1}, \mu=\delta_{a}$ and $m=3$. It is described by a single Feynman diagram, shown on the left of Fig 35.

Recall the dilogarithm function $\operatorname{Li}_{2}(z)$, its single valued version, the Bloch-Wigner function $\mathcal{L}_{2}(z)$, and the the cross-ratio $r\left(z_{1}, \ldots, z_{4}\right)$ of four points $z_{1}, \ldots, z_{4}$ on $\mathbb{P}^{1}$ normalized by $r(\infty, 0,1, z)=z$, see Section 1.3.

Lemma 11.1. One has

$$
G_{a}\left(a_{0}, a_{1}, a_{2}\right)=-\frac{1}{(2 \pi i)^{2}} \mathcal{L}_{2}\left(r\left(a, a_{0}, a_{1}, a_{2}\right)\right) .
$$

Proof. We may assume without loss of generality that $a=\infty$. Then, up to an additive constant, $G_{\infty}(x, y)=\log |x-y|$. Therefore

$$
G_{\infty}\left(a_{0}, a_{1}, a_{2}\right)=\frac{1}{(2 \pi i)^{3}} \int_{\mathbb{C P}^{1}} \omega_{2}\left(\log \left|x-a_{0}\right| \wedge \log \left|x-a_{1}\right| \wedge \log \left|x-a_{2}\right|\right) .
$$

It remains to use the differential equations for the Hodge correlator from Section 7 and $\mathcal{L}_{2}(z)$.

3. The classical polylogarithms. Let $X=\mathbb{C P}^{1}$ and $s_{0}=\infty$. Consider the following cyclic word of length $n+1$ :

$$
W_{n}=\mathcal{C}(\{1\} \otimes\{z\} \otimes\{0\} \otimes \ldots \otimes\{0\}) .
$$

A $W_{n}$-decorated Feynman diagram with an internal vertex incident to two $\{0\}$-decorated external vertices evidently contributes the zero integral. There is a unique $W_{n}$-decorated Feynman diagram with no internal vertices incident to two $\{0\}$ 's, see Fig 26. Denote by $\mathbf{L}_{n}(z)$ the Hodge correlator for $W_{n}$, which uses the clockwise orientation of the tree. We can package them into the generating series

$$
\mathbf{L}_{n}(z \mid u):=\sum_{n=0}^{\infty} \mathbf{L}_{n}(z) \frac{u^{n}}{n !} .
$$

Recall that the classical $n$-logarithm is a multivalued function on $\mathbb{C P}^{1}-\{0,1, \infty\}$, defined by an iterated integral:

$$
L i_{n}(z):=\int_{0 \leq x_{1} \leq \ldots \leq x_{n} \leq z} \frac{d x_{1}}{1-x_{1}} \wedge \frac{d x_{2}}{x_{2}} \wedge \ldots \wedge \frac{d x_{n}}{x_{n}} .
$$




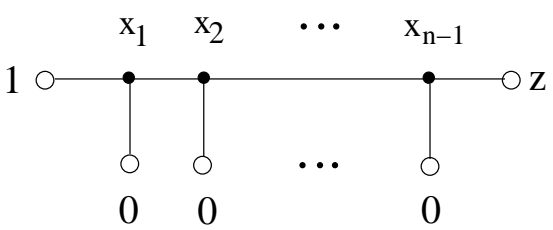

Figure 26: The Feynman diagram for the classical n-logarithm.

Here we integrate along a simplex consisting of points $\left(x_{1}, \ldots, x_{n}\right)$ on a path $\gamma$ connecting 0 and $z$, following each other on the path. It has a single-valued version ([Z1], $[\mathrm{BD}])$ :

$$
\mathcal{L}_{n}(z):=\begin{array}{ll}
\operatorname{Re} & (n: \text { odd }) \\
\operatorname{Im} & (n: \text { even })
\end{array}\left(\sum_{k=0}^{n-1} \beta_{k} \log ^{k}|z| \cdot L i_{n-k}(z)\right), \quad n \geq 2 .
$$

Here $\frac{2 x}{e^{2 x}-1}=\sum_{k=0}^{\infty} \beta_{k} x^{k}$, so $\beta_{k}=\frac{2^{k} B_{k}}{k !}$ where the $B_{k}$ are the Bernoulli numbers. For example $\mathcal{L}_{2}(z)$ is the Bloch - Wigner function.

Consider a modification $\mathbb{L}_{n}^{*}(z)$ of the function $\mathcal{L}_{n}(z)$ studied by A.Levin in $[\mathrm{L}]$ :

$$
\mathbb{L}_{n}^{*}(z):=4^{-(n-1)} \sum_{k \text { even; } 0 \leq k \leq n-2}\left(\begin{array}{c}
2 n-k-3 \\
n-1
\end{array}\right) \frac{2^{k+1}}{(k+1) !} \mathcal{L}_{n-k}(z) \log ^{k}|z| .
$$

It is also handy to have a slight modification of this function:

$$
\mathbb{L}_{n}(z)=4^{(n-1)}\left(\begin{array}{c}
2 n-2 \\
n-1
\end{array}\right)^{-1} \mathbb{L}_{n}^{*}(z)
$$

So $\mathbb{L}_{n}(z)=\mathcal{L}_{n}(z)$ if and only if $n \leq 3$.

Lemma 11.2. One has

$$
-\mathbf{L}_{n}(z)=(2 \pi i)^{-n} \mathbb{L}_{n}(z) .
$$

Proof. A direct integration carried out in Proposition 4.4.1 of [L] tells

$$
\begin{gathered}
-(2 \pi i)^{-n} \mathbb{L}_{n}^{*}(z)= \\
(2 \pi i)^{-(2 n-1)} \int_{\left(\mathbb{C P}^{1}\right)^{n-1}} \log \left|1-x_{1}\right| \bigwedge_{i=1}^{n-2}\left(d \log \left|x_{i}\right| \wedge d \log \left|x_{i}-x_{i+1}\right|\right) \wedge d \log \left|x_{n-1}\right| \wedge d \log \left|x_{n-1}-z\right| .
\end{gathered}
$$

By [GZ, Proposition 6.2] 10 given functions $\varphi_{i}$ on a complex $(n-1)$-dimensional manifold $M$,

$$
\int_{M} \omega_{2 n-2}\left(\varphi_{0} \wedge \ldots \wedge \varphi_{2 n-2}\right)=(-4)^{n-1}\left(\begin{array}{c}
2 n-2 \\
n-1
\end{array}\right)^{-1} \int_{M} \varphi_{0} d \varphi_{1} \wedge \ldots \wedge d \varphi_{2 n-2} .
$$

Using $\varphi_{i}=\log \left|f_{i}\right|$ we see that integral (253) coincides up to a factor with the Hodge correlator integral for the counterclockwise orientation of the tree on Fig 26. The factor $(-1)^{n-1}$ tells the difference between the counterclockwise and clockwise orientations of the tree.

\footnotetext{
${ }^{10}$ notice that we use the form $-\omega_{m}$ in [GZ] instead of $\omega_{m}$
} 
Alternatively, one can check using the differential equations for the function $\mathcal{L}_{n}(z)$ that the function $\mathbb{L}_{n}^{*}(z)$ satisfies the differential equation

$$
d \mathbb{L}_{n}^{*}(z)=\frac{2 n-3}{2 n-2} \mathbb{L}_{n-1}^{*}(z) d^{\mathbb{C}} \log |z|-\frac{1}{2 n-2} \log |z| d^{\mathbb{C}} \mathbb{L}_{n-1}^{*}(z) .
$$

The function $\mathbf{L}_{n}(z)$ is a Hodge correlator, and thus satisfies the same differential equation (Section 7), and is zero if $z=0$. This implies Proposition 11.2 by induction, since $\mathbb{L}_{n}^{*}(0)=0$.

So the Hodge correlator Cor $_{\mathcal{H}}$ delivers a single-valued version of the classical polylogarithm, while multiplying it by $4^{-(n-1)}\left(\begin{array}{c}2 n-2 \\ n-1\end{array}\right)$, i.e. using the normalized Hodge correlator Cor ${ }_{\mathcal{H}}^{*}$ (Section 7), we make the differential equation nicer.

4. Cyclic multiple polylogarithms and Hodge correlators. Let us set $s_{0}=\infty$, and

$$
\mathbb{L}_{k_{0} ; \ldots ; k_{m}}\left(a_{0}: \ldots: a_{m}\right):=\operatorname{Cor}_{\mathcal{H}, \infty}\left(\left\{a_{0}\right\} \otimes\{0\}^{\otimes k_{0}} \otimes \ldots \otimes\left\{a_{m}\right\} \otimes\{0\}^{\otimes k_{m}}\right), \quad a_{i} \in \mathbb{C}^{*} .
$$

We package these numbers into a generating series

$$
\mathbb{L}\left(a_{0}: \ldots: a_{m} \mid u_{0}: \ldots: u_{m}\right):=\sum_{k_{i}=0}^{\infty} \mathbb{L}_{k_{1} ; \ldots ; k_{m}}\left(a_{0}: \ldots: a_{m}\right) \frac{u_{0}^{k_{0}}}{k_{0} !} \ldots \frac{u_{m}^{k_{m}}}{k_{m} !} .
$$

The Hodge correlator integral assigned to the right diagram on Fig 27 equals $\mathbf{L}_{n}\left(a_{1} / a_{0}\right)$.
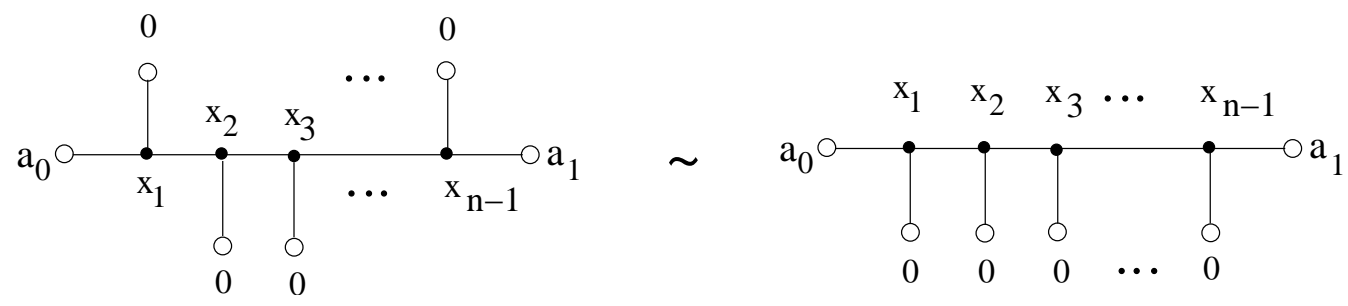

Figure 27: Feynman diagrams for the depth one Hodge correlators.

Lemma 11.3. One has

$$
\mathbb{L}\left(a_{0}: a_{1} \mid u_{0}: u_{1}\right)=\mathbf{L}\left(\frac{a_{1}}{a_{0}} \mid u_{1}-u_{0}\right) .
$$

Proof. The left hand side is a sum of the integrals attached to the diagrams on the left of Fig 27. The integral attached to the left Feynman diagram on Fig 27 coincides up to a sign, by its very definition, with the integral for the right Feynman diagram on Fig 27. The sign is

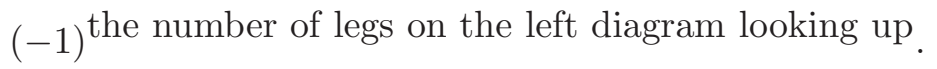

Equivalently, the exponent is the number of legs which we need to flip in order to transform one diagram to the other. Indeed, there is a natural bijection between the edges of these two graphs. It does not respect their canonical orientations. The sign measures the difference: flipping an edge we change the sign of the canonical orientation of the tree. It follows that

$$
\mathbb{L}\left(a_{0}: a_{1} \mid u_{0}: u_{1}\right) \stackrel{\text { def }}{=} \sum_{k_{0}, k_{1}=0}^{\infty} \mathbb{L}_{k_{0} ; k_{1}}\left(a_{0}: a_{1}\right) \frac{u_{0}^{k_{0}}}{k_{0} !} \frac{u_{1}^{k_{1}}}{k_{1} !}=
$$




$$
\sum_{k_{0}, k_{1}=0}^{\infty}(-1)^{k_{0}} \mathbf{L}_{k_{0}+k_{1}}\left(a_{0}: a_{1}\right) \frac{u_{0}^{k_{0}}}{k_{0} !} \frac{u_{1}^{k_{1}}}{k_{1} !}=\sum_{k=0}^{\infty} \mathbf{L}_{k}\left(a_{0}: a_{1}\right) \frac{\left(u_{1}-u_{0}\right)^{k}}{k !}=\mathbf{L}\left(\frac{a_{1}}{a_{0}} \mid u_{1}-u_{0}\right) .
$$

The second equality was explained above.

Cyclic multiple polylogarithms. We are going to define them, following Section 8 of [G1], as a yet another generating series

$$
\mathbf{L}\left(a_{0}: \ldots: a_{m} \mid t_{0}, \ldots, t_{m}\right), \quad a_{i} \in \mathbb{C}^{*}, \quad t_{i} \in H_{1}\left(\mathbb{C}^{*}, \mathbb{R}\right), \quad t_{0}+\ldots+t_{m}=0 .
$$

Consider a plane trivalent tree $T$ decorated by $m+1$ pairs $\left(a_{0}, t_{0}\right), \ldots,\left(a_{m}, t_{m}\right)$. We picture $a_{i}$ 's outside, and $t_{i}$ 's inside of the circle. Each oriented edge $\vec{E}$ of the tree $T$ provides an element
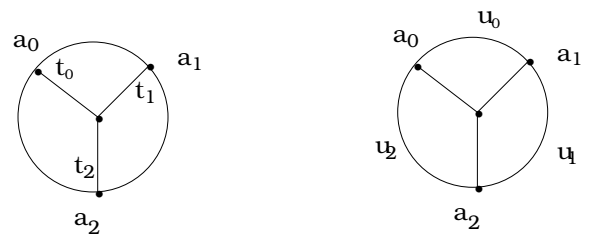

Figure 28: Decorations for the cyclic multiple polylogarithms.

$t(\vec{E})$ in $H_{1}\left(\mathbb{C}^{*}, \mathbb{R}\right)$ defined as follows. The edge $E$ determines two trees rooted at $E$, see Fig $[29$, An orientation of the edge $E$ allows to choose one of these trees: the one obtained by going in

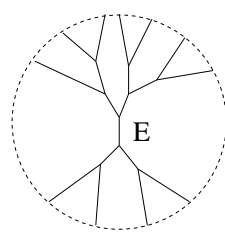

Figure 29: An edge $E$ of a tree provides two trees rooted at $E$.

the direction shown by the orientation. The union of the incoming (i.e. different from $E$ ) legs of these rooted trees coincides with the set of all legs of the initial tree. Let $t(\vec{E})$ be the sum of all $t_{i}$ 's corresponding to the incoming legs of one of these trees. The opposite orientation of the edge $E$ produces the element $-t(\vec{E})$. We define the generating series for the cyclic multiple polylogarithms just like a Hodge correlator, with the generating series (255) for the classical polylogarithms serving as Green functions. Namely, given a plane trivalent tree $T$ decorated as above, and an edge $E$ of $T$, let us define a generating series assigned to the pair $(E, T)$ :

$$
\mathbf{L}\left(x_{1}^{E}: x_{2}^{E} \mid t_{1}^{E}, t_{2}^{E}\right)
$$

We define first the arguments $x_{1}^{E}, x_{2}^{E}, t_{1}^{E}, t_{2}^{E}$. Choose an orientation of the edge $E$. It provides an ordered pair $\left(x_{1}^{E}, x_{2}^{E}\right)$ of the vertices of $E$, as well as a pair of vectors $\left(t_{1}^{E}, t_{2}^{E}\right):=(t(\vec{E}),-t(\vec{E}))$. Then $\mathbf{L}\left(x_{1}^{E}: x_{2}^{E} \mid t_{1}^{E}, t_{2}^{E}\right)$ is given by the generating series (255) evaluated at these arguments:

$$
\mathbf{L}\left(x_{1}^{E}: x_{2}^{E} \mid t_{1}^{E}, t_{2}^{E}\right):=\mathbf{L}\left(\frac{x_{1}^{E}}{x_{2}^{E}} \mid t_{1}^{E}\right) .
$$


Thanks to (255) and the symmetry $\mathbf{L}(a \mid t)=\mathbf{L}\left(a^{-1} \mid-t\right)$, the function $\mathbf{L}\left(x_{1}^{E}: x_{2}^{E} \mid t_{1}^{E}, t_{2}^{E}\right)$ does not change when we change the orientation of the edge $E$ :

$$
\mathbf{L}\left(x_{1}^{E}: x_{2}^{E} \mid t_{1}^{E}, t_{2}^{E}\right)=\mathbf{L}\left(x_{2}^{E}: x_{1}^{E} \mid t_{2}^{E}, t_{1}^{E}\right) .
$$

Now we are going to apply the map $\omega_{m}$ to the wedge product of the generating series (256) over the edges $E$ of the tree $T$. The map $\omega_{m}$ is usually applied, in the case of curves, to a wedge product of functions. In our case (256) is a generating series whose coefficients are functions, and we apply $\omega_{m}$ to the coefficients. Here is an example. Let $a(u):=\sum a_{i} u^{i}$ and $b(v):=\sum b_{j} v^{j}$ where $a_{i}, b_{j}$ are functions on a curve, and $u, v$ are formal variables. Then

$$
\omega_{2}(a(u), b(v))=\omega_{2}\left(\sum_{i} a_{i} u^{i}, \sum_{j} b_{j} v^{j}\right):=\sum_{i, j} \omega\left(a_{i}, b_{j}\right) u^{i} v^{j} .
$$

Then we integrate over the product of $\mathbb{C P}^{1}$ 's labeled by the internal vertices of $T$. Finally, we take the sum over all plane 3 -valent trees $T$ with the given decoration. We arrive at the following definition.

\section{Definition 11.4.}

$$
\begin{gathered}
\mathbf{L}\left(a_{0}: \ldots: a_{m} \mid t_{0}, \ldots, t_{m}\right):=(2 \pi i)^{-(2 m-1)} \sum_{T} \operatorname{sgn}\left(E_{1} \wedge \ldots \wedge E_{2 m-1}\right) . \\
\int_{\left(\mathbb{C P}^{1}\right)^{m-1}} \omega_{2 m-2}\left(\mathbf{L}\left(x_{1}^{E_{1}}: x_{2}^{E_{1}} \mid t_{1}^{E_{1}}, t_{2}^{E_{1}}\right) \wedge \ldots \wedge \mathbf{L}\left(x_{1}^{E_{2 m-1}}: x_{2}^{E_{2 m-1}} \mid t_{1}^{E_{2 m-1}}, t_{2}^{E_{2 m-1}}\right)\right) .
\end{gathered}
$$

Theorem 11.5. $\mathbb{L}\left(a_{0}: \ldots: a_{m} \mid u_{0}: \ldots: u_{m}\right)=\mathbf{L}\left(a_{0}: \ldots: a_{m} \mid u_{0}-u_{m}, u_{1}-u_{0}, \ldots, u_{m}-u_{m-1}\right)$.

Proof. The depth 1 case. This is Lemma 11.3. To proceed further, we need temporarily another generating series, whose combinatorics is illustrated on Fig 30 .

$$
\begin{aligned}
& \mathbb{L}^{\prime}\left(a_{0}: \ldots: a_{m} \mid s_{0}, t_{0} ; \ldots ; s_{m}, t_{m}\right):= \\
& \quad \sum_{n_{i}^{\prime}, n_{i}^{\prime \prime}=0}^{\infty} \mathbb{L}_{n_{0}^{\prime}, n_{0}^{\prime \prime} ; \ldots ; n_{m}^{\prime}, n_{m}^{\prime \prime}}\left(a_{0}: \ldots: a_{m}\right) \frac{s_{0}^{n_{0}^{\prime}}}{n_{0}^{\prime} !} \frac{t_{0}^{n_{0}^{\prime \prime}}}{n_{0}^{\prime \prime !}} \ldots \frac{s_{m}^{n_{m}}}{n_{m} !} \frac{t_{m}^{n_{m}^{\prime \prime}}}{n_{m}^{\prime \prime} !} .
\end{aligned}
$$

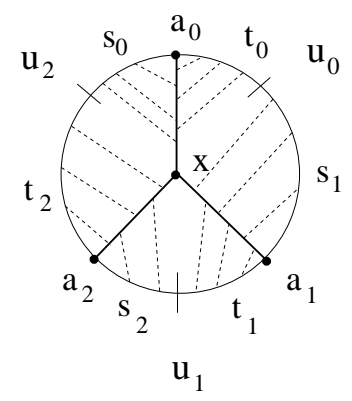

Figure 30: A Feynman diagram for the double polylogarithm.

The depth 2 case. The Hodge correlator integral is given by a Feynman diagram as on Fig 30. One has

$$
\mathbb{L}^{\prime}\left(a_{0}: a_{1}: a_{2} \mid u_{2}, u_{0} ; u_{0}, u_{1} ; u_{1}, u_{2}\right)=\mathbb{L}\left(a_{0}: a_{1}: a_{2} \mid u_{0}, u_{1}, u_{2}\right)
$$


Indeed, to get the right hand side we have to substitute $t_{0}=s_{1}=u_{0}, t_{1}=s_{2}=u_{1}, t_{2}=s_{0}=u_{0}$ into the sum (258), getting the left hand side. Furthermore, the same arguments about flipping the edges as in the proof of Lemma 11.3 show that

$$
\mathbb{L}^{\prime}\left(a_{0}: a_{1}: a_{2} \mid s_{0}, t_{0} ; s_{1}, t_{1} ; s_{2}, t_{2}\right)=\mathbf{L}\left(a_{0}: a_{1}: a_{2} \mid t_{0}-s_{0}, t_{1}-s_{1}, t_{2}-s_{2}\right) .
$$

Here we integrate first over the internal vertices incident to the external $\{0\}$-decorated vertices. Thanks to Lemma 11.3 we get the L-propagators assigned to the three "thick" edges of the tree. The remaining we get precisely the definition of the depth two function $\mathbf{L}$. Combining, we get

$$
\mathbb{L}\left(a_{0}: a_{1}: a_{2} \mid u_{0}, u_{1}, u_{2}\right)=\mathbf{L}\left(a_{0}: a_{1}: a_{2} \mid u_{0}-u_{2}, u_{1}-u_{0}, u_{2}-u_{1}\right) .
$$

The general case. It proceeds just like the depth 2 case. The case $m=3$ is illustrated on Fig [31. For example, we assign $u_{2}-u_{0}=\left(u_{2}-u_{1}\right)+\left(u_{1}-u_{0}\right)$ to the internal edge $E$ of the tree on Fig 31, in agreement with the recepee for $t(\vec{E})$.

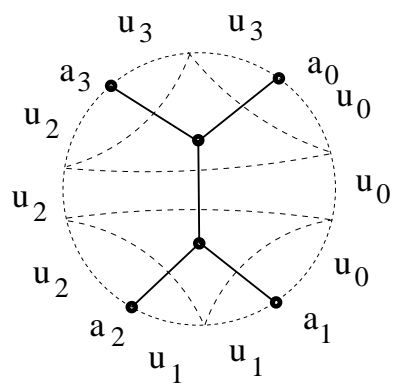

Figure 31: A Feynman diagram for the triple polylogarithm.

5. Double shuffle relations. We have defined the motivic cyclic multiple polylogarithms, denoted below $\mathrm{L}_{n_{0}, \ldots, n_{m}}^{\mathcal{M}}\left(a_{0}: \ldots: a_{m}\right)$, in (247). Recall that they are elements of the motivic Lie algebra of the category of mixed Tate motives. They are linear combinations of the traditional motivic multiple polylogarithms functions, but satisfy cleaner identities, as Lemma 11.6 shows.

When the ground field is $\mathbb{C}$, and elements $a_{i} \in \mathbb{C}$ vary, we can apply the Hodge realisation functor, followed by the canonical real period map. Then we get well defined real valued functions depending on the complex variables $a_{i}$. We prove that they are nothing else but the single valued cyclic multiple polylogarithm functions defined above. They, of course, satisfy all the identities which their motivic counterparts have.

The traditional weight $m$ motivic multiple polylogarithms $\mathrm{L}_{n_{1}, \ldots, n_{m}}^{\mathcal{M}}\left(a_{1}, \ldots, a_{m}\right)$ live in the motivic Hopf algebra. Therefore, when the elements $a_{i} \in \mathbb{C}$ vary, applying the Hodge realisation functor to them, we get a variation of framed mixed $\mathbb{Q}$-Hodge structures, which gives rise to a multivalued analytic function on the parameter space. Precisely, to get the functions we have to choose a splitting of the weight filtration, which exists only locally, and then use the framings.

Since motivic cyclic multiple polylogarithms live in the motivic Lie coalgebra rather then in the motivic Hopf algebra, they gives rise to multivalued analytic functions well defined only modulo products of similar lower weight functions. We would like to stress that, in contrast with this, the single valued cyclic multiple polylogarithms functions are well defined functions. 
Lemma 11.6. i) The cyclic multiple polylogarithms enjoy the dihedral symmetry relations

$$
\begin{gathered}
\mathbb{L}\left(a_{0}: \ldots: a_{m} \mid t_{0}: \ldots: t_{m}\right)=\mathbb{L}\left(a_{1}: \ldots: a_{m}: a_{0} \mid t_{1}: \ldots: t_{m}: t_{0}\right)= \\
(-1)^{m+1} \mathbb{L}\left(a_{m}: \ldots: a_{0} \mid t_{m}: \ldots: t_{0}\right) .
\end{gathered}
$$

and the shuffle relations

$$
\sum_{\sigma \in \Sigma_{p, q}} \mathbb{L}\left(a_{0}: a_{\sigma(1)} \ldots: a_{\sigma(m)} \mid t_{0}: t_{\sigma(1)}: \ldots: t_{\sigma(m)}\right)=0
$$

ii) The motivic cyclic multiple polylogs $\mathrm{L}^{\mathcal{M}}\left(a_{0}: \ldots: a_{m} \mid t_{0}: \ldots: t_{m}\right)$ satisfy the same relations.

Proof. i) The first is clear from the definition. The second follows from Proposition 3.5, which shows the shuffle relations for Hodge correlators. ii) is similar.

Remark. The shuffle relations (260) do not contain any products since the cyclic multiple polylogarithms by the very definition live in the motivic Lie coalgebra, and the products are killed by passing to the motivic Lie coalgebra.

The definitions below were motivated by the results of G4]. Set

$$
\begin{gathered}
\mathbb{L}\left(a_{0}: \ldots: a_{m}\right):=\mathbb{L}_{0, \ldots, 0}\left(a_{0}: \ldots: a_{m}\right), \\
\mathbb{L}\left(a_{0}, \ldots, a_{m}\right):=\mathbb{L}\left(1: a_{0}, a_{0} a_{1}, \ldots, a_{0} a_{1} \ldots a_{m-1}\right), \quad a_{0} \ldots a_{m}=1 .
\end{gathered}
$$

We also need their motivic versions $\mathrm{L}^{\mathcal{M}}\left(a_{0}: \ldots: a_{m}\right)$ and $\mathrm{L}^{\mathcal{M}}\left(a_{0}, \ldots, a_{m}\right)$.

Proposition 11.7. i) For any $p+q=m, p, q \geq 1$ one has the second shuffle relations

$$
\sum_{\sigma \in \Sigma_{p, q}} \mathbb{L}\left(a_{0}, a_{\sigma(1)}, \ldots, a_{\sigma(m)}\right)=0 .
$$

ii) The same is true for the $\mathcal{C}$-motivic multiple logarithms $\mathrm{L}^{\mathcal{M}}\left(a_{0}, \ldots, a_{m}\right)$.

Proof. ii) The elements $\mathrm{L}^{\mathcal{M}}\left(a_{0}: \ldots: a_{m}\right)$ satisfy the same coproduct formula as the generators of the dihedral Lie coalgebra, see (81) in [G4]:

$$
\delta \mathrm{L}^{\mathcal{M}}\left(a_{0}: \ldots: a_{m}\right)=\operatorname{Cycle}_{m+1}\left(\sum_{k=1}^{m-1} \mathrm{~L}^{\mathcal{M}}\left(a_{0}: \ldots: a_{k}\right) \wedge \mathrm{L}^{\mathcal{M}}\left(a_{k}: \ldots: a_{m}\right)\right) .
$$

Indeed, this formula is equivalent to the one for the differential $\delta_{S}$ in Section 6,2, which was made motivic in Section 10.

Let us prove the shuffle relations by the induction on $m$. Denote by $s_{2}^{p, q}\left(a_{0}, \ldots, a_{m}\right)$ the left hand side in (261). Theorem 4.3 in [G4 tells that these elements span a coideal. This implies by the induction that $\delta s_{2}^{p, q}\left(a_{0}, \ldots, a_{m}\right)=0$. Therefore $s_{2}^{p, q}\left(a_{0}, \ldots, a_{m}\right) \in \operatorname{Ext}^{1}(\mathbb{Q}(0), \mathbb{Q}(m))$ in the category of variations over the base parametrising the $a_{i}$ 's. Since $m \geq 2, \operatorname{Ext}^{1}(\mathbb{Q}(0), \mathbb{Q}(m))$ is rigid, and thus is a constant. Setting $a_{i}=1$ in (260) we get $\mathrm{L}^{\mathcal{M}}(1: \ldots: 1)=0$. Thus $s_{2}^{p, q}(1, \ldots, 1)=0$, and hence the constant above is zero. The statement is proved.

i) Follows from ii) by applying the period map, or by using the differential equations. 
So the cyclic multiple logarithms as well as their motivic avatars satisfy the double shuffle relations from Section 4 of [G4 on the nose, without lower depth corrections or products. The absence of the products is a general feature of the Lie-type elements. It is also a general feature of the Lie-type periods, since the latter are obtained by applied the period map to the Hodge counterpart of the motivic Lie coalgebra.

The absence of the lower depth corrections is a remarkable fact. It implies that there is a homomorphism from the diagonal part of the dihedral Lie coalgebra of $\mathbb{C}^{*}$ or $F^{*}$ (loc. cit.) to the motivic Lie coalgebra which sends the standard generators of the former to the motivic cyclic multiple logarithms. Notice that the construction of a similar homomorphism in G4 required the associate graded for the depth filtration of the motivic Lie coalgebra. Specifying to the roots of unity, we conclude that the mysterious connection between the geometry of modular varieties and motivic cyclic multiple logarithms at roots of unity is valid without going to the associated graded for the depth filtration.

So the generators of the dihedral Lie coalgebras are related to the cyclic multiple polylogarithms, rather then the usual multiple polylogarithms, which differ by the lower depth terms.

Remark. The double shuffle relations for the depth 2 motivic cyclic multiple polylogarithms follow from (259), since in this case they are equivalent to the dihedral symmetry relations. Their proof in general is more involved since the coproduct of the motivic cyclic multiple polylogarithms has the lower depth terms.

\subsection{Hodge correlators on elliptic curves are multiple Eisenstein-Kronecker series}

1. The classical Eisenstein-Kronecker series. Let $E$ be a complex elliptic curve with a lattice of periods $\Gamma$, so that $E=\mathbb{C} / \Gamma$. The intersection form $\Lambda^{2} \Gamma \rightarrow 2 \pi i \mathbb{Z}$ leads to a pairing $\chi: E \times \Gamma \rightarrow S^{1}$. So a point $a \in E$ provides a character $\chi_{a}: \Gamma \longrightarrow S^{1}$. We denote by $d z$ the differential on $E$ provided by the coordinate $z$ on $\mathbb{C}$. We normalize the lattice so that $\Gamma=\mathbb{Z} \oplus \mathbb{Z} \tau$, $\operatorname{Im} \tau>0$.

Consider the generating series for the classical Eisenstein-Kronecker series

$$
K(a \mid t):=\frac{\tau-\bar{\tau}}{2 \pi i} \sum_{\gamma \in \Gamma-\{0\}} \frac{\chi_{a}(\gamma)}{|\gamma-t|^{2}} .
$$

It depends on a point $a$ of the elliptic curve and an element $t$ in a formal neighborhood of zero in $H_{1}(E, \mathbb{R})$. It is invariant under the involution $a \longmapsto-a, t \longmapsto-t$. Expanding it into the power series in $t$ and $\bar{t}$ we get the classical Eisenstein-Kronecker series as the coefficients:

$$
K(a \mid t)=\sum_{p, q \geq 1}\left(\frac{\tau-\bar{\tau}}{2 \pi i} \sum_{\gamma \in \Gamma-\{0\}} \frac{\chi_{a}(\gamma)}{\gamma^{p} \bar{\gamma}^{q}}\right) t^{p-1} \bar{t}^{q-1} .
$$

Set $\operatorname{Sym}_{p+q} F\left(x_{1}, \ldots, x_{p+q}\right):=\sum_{\sigma} F\left(x_{\sigma(1)}, \ldots, x_{\sigma(p+q)}\right)$. Consider the following cyclic word:

$$
W_{p, q}:=\mathcal{C}\left(\{0\} \otimes\{a\} \otimes \frac{d \bar{z}^{p}}{p !} \cdot \frac{d z^{q}}{q !}\right):=\operatorname{Sym}_{p+q} \mathcal{C}\left(\{0\} \otimes\{a\} \otimes \frac{d \bar{z}^{\otimes p}}{p !} \otimes \frac{d z^{\otimes q}}{q !}\right) .
$$

The corresponding Hodge correlator is given by the Feynman diagram on Fig 32 , 


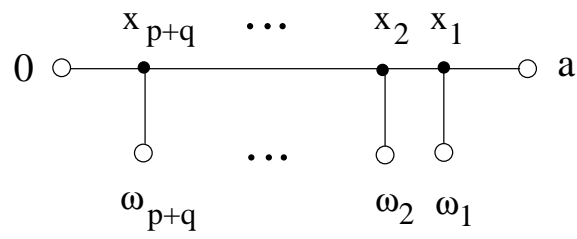

Figure 32: A Feynman diagram for the classical Eisenstein-Kronecker series; $\omega_{i}=d z$ or $d \bar{z}$.

Proposition 11.8. i) The Hodge correlator of $W_{p, q}$ is the classical Eisenstein-Kronecker series:

$$
\operatorname{Cor}_{\mathcal{H}}\left(W_{p, q}\right)=\frac{(-1)^{p}}{2 \pi i}\left(\frac{\tau-\bar{\tau}}{2 \pi i}\right)^{p+q+1} \sum_{\gamma \neq 0} \frac{\chi_{a}(\gamma)}{\gamma^{p+1} \bar{\gamma}^{q+1}} .
$$

ii) The function $K(a \mid t)$ coincides with the generating function of the Hodge correlators of 264).

Proof. i) The normalized Green function $g(x, y)$ for the invariant volume form $\operatorname{vol}_{E}$ on $E$ is a distribution given by $g(x, y):=g(x-y)$ where

$$
\chi_{z}(\gamma):=\exp \left(\frac{2 \pi i(z \bar{\gamma}-\bar{z} \gamma)}{\tau-\bar{\tau}}\right), \quad g(z):=\frac{\tau-\bar{\tau}}{2 \pi i} \sum_{\gamma \neq 0} \frac{\chi_{z}(\gamma)}{|\gamma|^{2}}, \quad(2 \pi i)^{-1} \bar{\partial} \partial g(z)=\delta_{0}-\operatorname{vol}_{E} .
$$

Therefore the Hodge correlator of (264) is obtained by integrating the following form over $E^{p+q}$ :

$$
\Omega_{p, q}:=\frac{(-1)^{p}}{(2 \pi i)^{(p+q+1)}} g\left(a-x_{1}\right) \bigwedge_{s=1}^{p} d \bar{x}_{s} \wedge \partial g\left(x_{s}-x_{s+1}\right) \wedge \bigwedge_{t=1}^{q} d x_{p+t} \wedge \bar{\partial} g\left(x_{p+t}-x_{p+t+1}\right) .
$$

Here $x_{p+q+1}:=0$. We used the clockwise orientation of the tree to make the product. Notice that the component of the form $\omega_{p+q}$ responsible for this form comes with the coefficient $(-1)^{p} p ! q ! /(p+q)$ !. The integral does not depend on the normalization of the Green function. The term by term differentiation of the sum defining the distribution $g(z)$ in (265) gives

$$
\partial \log g(z)=\sum_{\gamma \neq 0} \frac{\chi_{z}(\gamma)}{\gamma} d z, \quad \bar{\partial} g(z)=-\sum_{\gamma \neq 0} \frac{\chi_{z}(\gamma)}{\bar{\gamma}} d \bar{z}
$$

Thus we get

$$
\int_{E^{p+q}} \Omega_{p, q}=\frac{(-1)^{p}}{2 \pi i}\left(\frac{\tau-\bar{\tau}}{2 \pi i}\right)^{p+q+1} \sum_{\gamma \neq 0} \frac{\chi_{a}(\gamma)}{\gamma^{p+1} \bar{\gamma}^{q+1}} .
$$

Indeed, we have a convolution of the Green function with its first derivatives, which amounts to a product after the Fourier transform.

ii) Let $l, \bar{l} \in H_{1}(E, \mathbb{C})$ be the homology classes dual to $d z$ and $d \bar{z}$. The Hodge correlators of the elements (264) are naturally packaged into a generating function

$$
\frac{(-1)^{p}}{2 \pi i} \frac{\tau-\bar{\tau}}{2 \pi i} \sum_{p, q=0}^{\infty}\left(\frac{\tau-\bar{\tau}}{2 \pi i}\right)^{p+q} \sum_{\gamma \neq 0} \frac{\chi_{a}(\gamma)}{\gamma^{p+1} \bar{\gamma}^{q+1}} \bar{l}^{p} l^{q} .
$$

This is naturally a function on a formal neighborhood of the origin in $H_{D R}^{1}(E, \mathbb{C})(1)^{+}$, where + means the complex conjugation acting on the forms on $E$. On the other hand $K(a \mid t)$ is naturally 
a function on $t \in \mathbb{C}=H_{1}(\Gamma) \otimes \mathbb{R}=H_{1}(E, \mathbb{R})$. Indeed, $\gamma$ and $t$ in formula (262) lie in the same space!

The intersection pairing $\langle *, *\rangle$ on $H_{1}$ provides an isomorphism $i: H_{D R}^{1}(E, \mathbb{C})(1)^{+} \rightarrow H_{1}(E, \mathbb{R})$ such that $\langle i(\omega), h\rangle:=(\omega, h)$. It follows that

$$
\langle l, \bar{l}\rangle=\frac{2 \pi i}{\tau-\bar{\tau}}, \quad i: d z \longmapsto t:=-\frac{\tau-\bar{\tau}}{2 \pi i} \bar{l}, \quad i: d \bar{z} \longmapsto \bar{t}:=\frac{\tau-\bar{\tau}}{2 \pi i} l .
$$

Using this we get

$$
(\underline{266)})=\frac{1}{2 \pi i} \frac{\tau-\bar{\tau}}{2 \pi i} \sum_{\gamma \neq 0} \frac{\chi_{a}(\gamma)}{|\gamma-t|^{2}}=\frac{1}{2 \pi i} K(a \mid t) \text {. }
$$

Example. It is easy to see that when $a \in E$ is a torsion point, the coproduct of the averaged base point motivic correlator of $\mathcal{C}\left(\{0\} \otimes\{a\} \otimes \mathrm{Sym}^{n} \mathbb{H}\right.$ ) (Section 10.5 4) is zero. So we get an element

$$
\operatorname{Cor}_{E-E_{\text {tors }}}^{0}\left(\mathcal{C}\left(\{0\} \otimes\{a\} \otimes \operatorname{Sym}^{n} \mathbb{H}\right)\right) \in \operatorname{Ext}_{\mathcal{C}}^{1}\left(\mathbb{Q}(0), \operatorname{Sym}^{n} \mathbb{H}(1)\right) .
$$

Its real period is given by the Eisenstein-Kronecker series. When $E$ is a CM curve it gives the special values of the Hecke L-series with Grössencharacters. Summarizing, we get the motivic cohomology class corresponding to this special value. See [B], Den1, [BL for different approaches.

2. Hodge correlators on an elliptic curve. Let $\mu$ be the unique invariant volume form on $E$ of volume 1. The Green function corresponding to $\mu$ is normalized by

$$
\int_{E(\mathbb{C})} G_{\mu}(s, z) d z \wedge d \bar{z}=0 .
$$

Below we consider the Hodge correlators decorated by the elements of

$$
\mathcal{C}\left(\left\{a_{0}\right\} \otimes S^{k_{0}}\left(\Omega_{E}^{1} \oplus \bar{\Omega}_{E}^{1}\right) \otimes \ldots \otimes\left\{a_{m}\right\} \otimes S^{k_{m}}\left(\Omega_{E}^{1} \oplus \bar{\Omega}_{E}^{1}\right)\right) .
$$

The symmetric Hodge correlators on an elliptic curve are linear combinations of those which appear when $\left\{a_{i}\right\}$ are replaced by degree zero divisors on $E$. Abusing language, we call any Hodge correlator on an elliptic curve decorated by (269) and defined by using the normalized Green function $G_{\mu}(x, y)$ a symmetric Hodge correlator. The depth of the Hodge correlator of $W$ is the number of the $S$-factors of $W$ minus one.

We package depth $m$ symmetric Hodge correlators on $E$ into non-holomorphic generating series:

$$
\begin{aligned}
K_{p_{0}, q_{0} ; \ldots ; p_{m}, q_{m}}\left(a_{0}: \ldots: a_{m}\right) & :=\operatorname{Cor}_{\mathcal{H}} \mathcal{C}\left(\left\{a_{0}\right\} \otimes \frac{d z^{p_{0}} d \bar{z}^{q_{0}}}{p_{0} ! q_{0} !} \otimes \ldots \otimes\left\{a_{m}\right\} \otimes \frac{d z^{p_{m}} d \bar{z}^{q_{m}}}{p_{m} ! q_{m} !}\right) . \\
K\left(a_{0}: \ldots: a_{m} \mid l_{0}: \ldots: l_{m}\right) & =\sum_{p_{i}, q_{i} \geq 0} K_{p_{0}, q_{0} ; \ldots ; p_{m}, q_{m}}\left(a_{0}: \ldots: a_{m}\right) \bar{l}_{0}^{p_{0}} l_{0}^{q_{0}} \ldots \bar{l}_{m}^{p_{m}} l_{m}^{q_{m}} .
\end{aligned}
$$

Following (267), we introduce the variables

$$
t_{i}:=-\frac{\tau-\bar{\tau}}{2 \pi i} \bar{l}_{i}, \quad \bar{t}_{i}:=\frac{\tau-\bar{\tau}}{2 \pi i} l_{i} . \quad t_{i}, \bar{t}_{i} \in H_{1}(E, \mathbb{R}) .
$$


3. The multiple Eisenstein-Kronecker series. Our next goal is to present the symmetric Hodge correlators on elliptic curves as integrals of the generating series $K(a \mid t)$. We rewrite the function (263) as

$$
\mathbf{K}\left(a_{1}: a_{2} \mid t_{1}, t_{2}\right):=K\left(a_{1}-a_{2} \mid t_{1}\right) ; \quad t_{1}+t_{2}=0 .
$$

Let us define, following Section 8 of [G1], the depth $m$ multiple Eisenstein-Kronecker series

$$
\mathbf{K}\left(a_{0}: \ldots: a_{m} \mid t_{0}, \ldots, t_{m}\right) ; \quad a_{i} \in E, \quad t_{i} \in H_{1}(E, \mathbb{R}), \quad t_{0}+\ldots+t_{m}=0,
$$

Consider a plane trivalent tree $T$, see Fig 28 , decorated by $m+1$ pairs $\left(a_{0}, t_{0}\right), \ldots,\left(a_{m}, t_{m}\right)$. Each oriented edge $\vec{E}$ of the tree $T$ provides an element $t(\vec{E}) \in H_{1}(E, \mathbb{R})$ just like in Section 10.1. We define the multiple Eisenstein-Kronecker series just like the multiple Green functions, with the generating series (271) for the classical Eisenstein-Kronecker series serving as Green functions:

$$
\begin{aligned}
& \mathbf{K}\left(a_{1}: \ldots: a_{m+1} \mid t_{1}, \ldots, t_{m+1}\right):=(2 \pi i)^{-(2 m-1)} \sum_{\mathrm{T}} \operatorname{sgn}\left(E_{1} \wedge \ldots \wedge E_{2 m-1}\right) . \\
& \int_{E^{m-1}} \omega_{2 m-2}\left(\mathbf{K}\left(x_{1}^{E_{1}}: x_{2}^{E_{1}} \mid t_{1}^{E_{1}}, t_{2}^{E_{1}}\right) \wedge \ldots \wedge \mathbf{K}\left(x_{1}^{E_{2 m-1}}: x_{2}^{E_{2 m-1}} \mid t_{1}^{E_{2 m-1}}, t_{2}^{E_{2 m-1}}\right)\right) .
\end{aligned}
$$

Here $\mathbf{K}\left(x_{1}^{E}: x_{2}^{E} \mid t_{1}^{E}, t_{2}^{E}\right)$ is the function (271) assigned to the edge $E$, defined just like in the rational case. The sum in (272) is over all plane 3 -valent trees $T$ cyclically labeled by the pairs $\left(a_{i}, t_{i}\right)$. The integral is over the product of copies of $E$ labeled by the internal vertices of $T$.

Clearly

$$
\mathbf{K}\left(a+a_{0}: \ldots: a+a_{m} \mid t_{0}, \ldots, t_{m}\right)=\mathbf{K}\left(a_{0}: \ldots: a_{m} \mid t_{0}, \ldots, t_{m}\right) .
$$

The multiple Eisenstein-Kronecker series enjoy the dihedral symmetry relations (259) and the shuffle relations (260) It is proved just the same way as Lemma 11.6.

Theorem 11.9. The generalized Eisenstein-Kronecker series are the generating series for the symmetric Hodge correlators on the elliptic curve E:

$$
K\left(a_{0}: \ldots: a_{m} \mid u_{0}: \ldots: u_{m}\right):=\mathbf{K}\left(a_{0}: \ldots: a_{m} \mid u_{m}-u_{0}, u_{0}-u_{1}, \ldots: u_{m-1}-u_{m}\right) .
$$

We visualize this as follows. The points $a_{i}$ sit on a circle, and cut it into $m+1$ arcs. We assign the variables $u_{i}$ to the $\operatorname{arcs}$ so that the vertex $a_{i}$ shares the arcs labeled by $u_{i-1}$ and $u_{i}$, see Fig 33 .
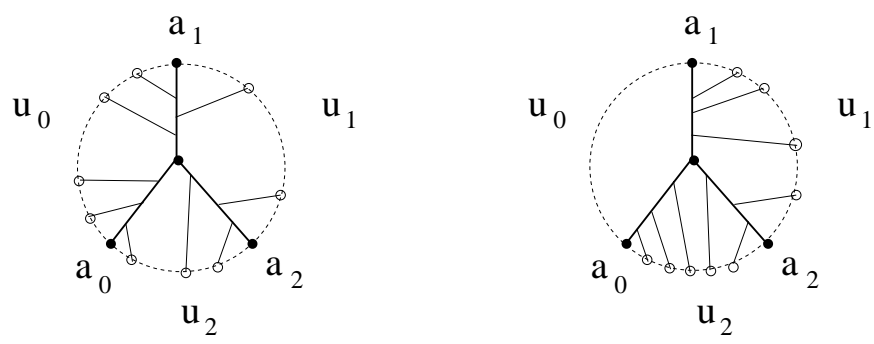

Figure 33: Feynman diagrams for symmetric Hodge correlators on an elliptic curve. 
Proof. A Feynman diagram decorated by a vector in (269), and which has an internal vertex incident to two $\omega$-decorated external vertices, contributes zero. Indeed, the correlator integral involves $\int_{E}\left(\omega_{1} \wedge \omega_{2}+\omega_{2} \wedge \omega_{1}\right)=0$. It follows that the only Feynman diagrams contributing to the Hodge correlators we consider are as on Fig 33 .

Symmetric Hodge correlators of depths -1 and 0 are zero. Indeed, every Feynman diagram with less then two $S$-decorated vertices has an internal vertex sharing two $\omega$-decorated edges.

The depth 1 case follows from Proposition 11.8. There are other depth 1 symmetric cyclic words, similar to the one on the left of Fig 27, where $\{0\}$ 's are replaced by $\omega_{i}$ 's. The integral for each of them coincides, up to a sign, with the one for the standard diagram on Fig 32 . Namely, just flip the $\omega$-decorated edges looking up, thus transforming the diagram to the one on Fig 32 .

The rest of the proof repeats the proof of Theorem 11.5 with a slight modification stemming from the fact that $H_{1}(E, \mathbb{R})$ is two-dimensional.

Remark. Let us show directly that the generating series (270) are invariant under the shift $u_{i} \longmapsto u_{i}-u_{0}$. We use the same argument as in the proof of Lemma 11.3. Each external edge touching the arc labeled by $u_{0}$ is flipped by moving its external vertex to the other arcs. For example, the right picture on Fig 33 represents the result of flipping of all external edges touching the $u_{0}$-arc on the left one. The correlator integral changes the sign under a flip of a single edge. Indeed, a flip changes only the orientation of the tree. This proves the claim.

4. Polylogarithms on curves. It is naturally to define polylogarithms on curves as the motivic/Hodge correlators of depth one. For the rational and elliptic curves we get this way the classical and elliptic polylogarithms. For the higher genus curves there is a yet smaller class of functions, defined in G1 and discussed below. Their motivic avatars, however, do not span a Lie coalgebra.

Let $X$ be a smooth compact complex curve of genus $g \geq 1$. and $\mu$ is a volume one measure on $X$. Set

$$
\mathbb{H}_{\mathbb{R}}:=H_{1}(X, \mathbb{R}), \quad \mathbb{H}_{\mathbb{C}}:=\mathbb{H}_{\mathbb{R}} \otimes \mathbb{C}=\mathbb{H}_{-1,0} \oplus \mathbb{H}_{0,-1} .
$$

For each integer $n \geq 1$ we define a 0 -current $G_{n}(x, y ; \mu)$ on $X \times X$ with values in

$$
\operatorname{Sym}^{n-1} \mathbb{H}_{\mathbb{C}}(1)=\oplus_{s+t=n-1} S^{p} \mathbb{H}_{-1,0} \otimes S^{q} \mathbb{H}_{0,-1} \otimes \mathbb{R}(1) .
$$

The current $G_{1}(x, y ; \mu)$ is the Green function $G_{\mu}(x, y)$, The current $G_{n}(x, y ; \mu)$ for $n>1$ is given by a function on $X \times X$. To get complex valued functions out of the vector function $G_{n}(x, y ; \mu)$ we proceed as follows. Let

$$
\bar{\Omega}_{p} \in S^{p} \bar{\Omega}^{1}, \quad \Omega_{q} \in S^{q} \Omega^{1} .
$$

Then $\bar{\Omega}_{p} \otimes \Omega_{q}$ is an element of the dual to (274) . Let us define the pairing $\left\langle G_{n}(x, y ; \mu), \bar{\Omega}_{p} \cdot \Omega_{q}\right\rangle$. Let

$$
\Omega_{p}=\omega_{\alpha_{1}} \cdot \ldots \cdot \omega_{\alpha_{p}}, \quad \Omega_{q}=\omega_{\beta_{1}} \cdot \ldots \cdot \omega_{\beta_{q}} ; \quad \omega_{*} \in \Omega^{1} .
$$

Denote by $p_{i}: X^{n-1} \longrightarrow X$ the projection on $i$-th factor.

Definition 11.10. The $n$-logarithm on the curve $X$ is given by

$$
\begin{gathered}
\left\langle G_{n}(x, y ; a), \bar{\Omega}_{p} \cdot \Omega_{q}\right\rangle:= \\
\frac{1}{(2 \pi i)^{n}} \operatorname{Alt}_{\left\{t_{1}, \ldots, t_{n-1}\right\}}\left(\int_{X^{n-1}} \omega_{n-1}\left(G\left(x, t_{1}\right) \wedge G\left(t_{1}, t_{2}\right) \wedge \ldots \wedge G\left(t_{n-1}, y\right)\right) \wedge \bigwedge_{i=1}^{p} p_{i}^{*} \bar{\omega}_{\alpha_{i}} \wedge \bigwedge_{j=1}^{t} p_{p+j}^{*} \omega_{\beta_{j}}\right) .
\end{gathered}
$$


Here we skewsymmetrize the integrand with respect to $t_{1}, \ldots, t_{n-1}$. Before the skewsymmetrization the integrand depends on the element $\bar{\omega}_{\alpha_{1}} \otimes \ldots \otimes \bar{\omega}_{\alpha_{p}}$ from $\otimes{ }^{p} \bar{\Omega}^{1}$; after it depends only on its image in $S^{p} \bar{\Omega}^{1}$; similarly for the second factor.

It is the Hodge correlator for the Feynman diagram on Fig 32 . For example, the dilogarithm on a curve $X$ is described by the second Feynman diagram on Fig 35. It is given by the integral

$$
\left\langle G_{2}(x, y ; \mu), \bar{\omega}_{\alpha}\right\rangle:=\frac{1}{(2 \pi i)^{2}} \quad \int_{X} \omega_{1}\left(G_{\mu}(x, t) \wedge G_{\mu}(t, y)\right) \wedge \bar{\omega}_{\alpha}(t) .
$$

Remark. Unlike in the genus $\leq 1$ case, there are non-trivial Hodge correlators of depths -1 and 0 . The simplest depth -1 Hodge correlator is illustrated on Fig 34. Furthermore, there are other depth one Hodge correlators then the polylogarithms defined above.

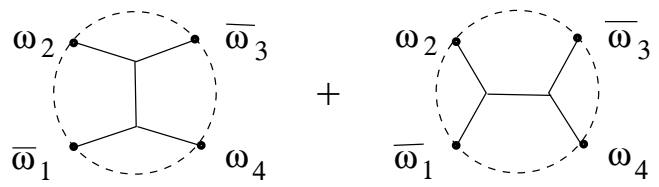

Figure 34: The simplest depth -1 Hodge correlator.

Just like in the rational and elliptic cases, one can introduce cyclic multiple polylogarithms by taking the generating function of the polylogarithms on curves as the propagator assigned to an edge of a trivalent tree, and repeating the construction of Definition 11.4.

One proves just the same way as in Theorem 11.5, that these way we get all Hodge correlators corresponding the elements of $\mathcal{C}\left(\left\{a_{0}\right\} \otimes S^{k_{0}}\left(\Omega_{X}^{1} \oplus \bar{\Omega}_{X}^{1}\right) \otimes \ldots \otimes\left\{a_{m}\right\} \otimes S^{k_{m}}\left(\Omega_{X}^{1} \oplus \bar{\Omega}_{X}^{1}\right)\right)$.

However, unlike in the cases of rational or elliptic curves, we do not get all Hodge correlators on curves this way, and the corresponding motivic correlators are not closed under the coproduct. The reason is that when $E$ is an elliptic curve, the direct summands of the twisted symmetric powers $\operatorname{Sym}^{m} H^{1}(E)(n)$ of the motive $H^{1}(E)$ give all simple object of the abelian tensor category of pure motives generated by $H^{1}(E)$. However when $X$ is regular projective curve of genus $\geq 2$, the direct summands of $\mathrm{Sym}^{m} H^{1}(X)(n)$ do not deliver all simple objects of the tensor category generated by $H^{1}(X)$.

\section{Motivic correlators on modular curves}

In the Section $X$ is a modular curve, and $S$ is the set of cusps.

\subsection{Hodge correlators on modular curves and generalized Rankin-Selberg integrals}

Rankin-Selberg integrals as Hodge correlators on modular curves. For an arbitrary curve $X$, there are three different types of the Hodge correlators for the length three cyclic words. They correspond to the three Feynman diagrams on Fig. 35. The first is the double Green function from Section 10.1. Let us interpret the other two in the case when $X$ is a modular curve, and $S$ is the set of its cusps. Let $\omega$ and $\omega_{1}, \omega_{2}$ be cuspidal weight two Hecke eigenforms. They are holomorphic 1-forms on $X$. Let $s \in S$ be a cuspidal divisor on the modular curve 


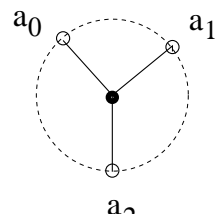

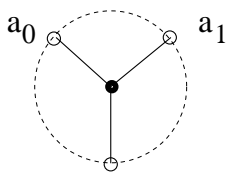

$\omega_{2}$

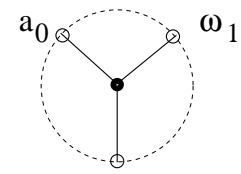

$\omega_{2}$

Figure 35: The three possible Feynman diagrams for the $m=2$ correlators.

$X$. Then $G(s, t)$ is an Eisenstein series, see, for example [GZ]. By the Manin-Drinfeld theorem [Ma]-Dr3 if $\operatorname{deg}(s)=0$, there exists a rational function $g_{s}$ on $X \operatorname{such}$ that $\operatorname{div} g_{s}=N \cdot s$. So $G(s, t)=N \log \left|g_{s}\right|^{2}$. Setting $a \sim \mathbb{Q}^{*} b$ if $a / b \in \mathbb{Q}^{*}$, we get

$$
\begin{aligned}
& \operatorname{Cor}_{\mathcal{H}}\left(\left(\left\{a_{0}\right\} \otimes\left\{a_{1}\right\} \otimes \omega\right)\right)=\int_{X(\mathbb{C})} G\left(a_{0}, t\right) d^{\mathbb{C}} G\left(a_{1}, t\right) \wedge \omega \sim \sim_{\mathbb{Q}^{*}} \int_{X(\mathbb{C})} \log \left|g_{a_{0}}\right| d^{\mathbb{C}} \log \left|g_{a_{1}}\right| \wedge \omega, \\
& \operatorname{Cor}_{\mathcal{H}}\left(\mathcal{C}\left(\left\{a_{0}\right\} \otimes \omega_{1} \otimes \bar{\omega}_{2}\right)\right)=\int_{X(\mathbb{C})} G\left(a_{0}, t\right) \omega_{1} \wedge \bar{\omega}_{2} \sim \sim_{\mathbb{Q}^{*}} \int_{X(\mathbb{C})} \log \left|g_{a}\right| \omega \wedge \bar{\omega} .
\end{aligned}
$$

Let $L(\omega, s)$ be the $L$-function of the rank two weight -1 motive $M_{\omega}$ corresponding to the Hecke eigenform $\omega$, and $L\left(\omega_{1} \otimes \omega_{2}, s\right)$ the $L$-function of the product of motives corresponding to different Hecke eigenforms $\omega_{1}$ and $\omega_{2}$. According to the classical Rankin-Selberg method the first integral is proportional, up to some standard factors, to $L(\omega, 2)$, and the second one to $L\left(\omega_{1} \otimes \omega_{2}, 2\right)$. Moreover, there exist degree zero divisors $a_{0}, a_{1}$ such that the proportionally coefficient is not zero $[\mathrm{B}]$.

Generalized Rankin-Selberg integrals. Let $X$ be a modular curve, given together with its uniformization $p_{X}: \mathcal{H} \rightarrow X$ by the hyperbolic plane $\mathcal{H}$. Choose a hyperbolic metric of curvature -1 on $X$. Denote by $\mu_{X}$ the corresponding volume 1 volume form on $X$. So $p_{X}^{*} \mu_{X}$ is the standard volume form $d x d y / y^{2}$ on the hyperbolic plane. Therefore if $\pi: Y \rightarrow X$ is a natural map of modular curves, i.e. a map commuting with the uniformization maps, then the volume forms $\mu_{X}$ and $\mu_{Y}$ are compatible: $\pi_{*} \mu_{Y}=\mu_{X}$.

Lemma 12.1. Let $\pi: Y \rightarrow X$ be a map of curves and $\pi_{*}\left(\mu_{Y}\right)=\mu_{X}$ for certain measures $\mu_{Y}, \mu_{X}$ on them. Let $\pi_{1}: Y \times Y \rightarrow X \times Y$ and $\pi_{2}: X \times Y \rightarrow X \times X$ be two natural maps. Then

$$
\pi_{1 *} G_{\mu_{Y}}\left(y_{1}, y_{2}\right)=\pi_{2}^{*} G_{\mu_{X}}\left(x_{1}, x_{2}\right)+C
$$

Proof. The Green function is defined uniquely up to adding a constant by the differential equation (74). The latter involves the volume form and the identity maps on the cohomology realized by the delta-currents $\delta_{\Delta}$ and the Casimir elements for the space of holomorphic/antiholomorphic 1-forms. Clearly $\pi_{1 *} \delta_{\Delta_{Y}}=\pi_{2}^{*} \delta_{\Delta_{X}}$. The latter fact implies a similar identity for the Casimirs. Finally,

$$
\begin{aligned}
& \pi_{1 *}\left(1_{Y} \otimes \mu_{Y}\right)=\operatorname{deg} \pi\left(1_{X} \otimes \mu_{Y}\right)=\pi_{2}^{*}\left(1_{X} \otimes \mu_{X}\right) ; \\
& \pi_{1 *}\left(\mu_{Y} \otimes 1_{Y}\right)=\mu_{X} \otimes 1_{Y}=\pi_{2}^{*}\left(\mu_{X} \otimes 1_{X}\right) .
\end{aligned}
$$


We set $\mathbb{V}_{X, S}:=H^{1}(X) \oplus \mathbb{Q}[S](-1)$.

We say that Green functions $G_{\mu_{Y}}$ and $G_{\mu_{X}}$ are compatible if the constant in Lemma 12.1 is zero. Choose a compatible collection of Green functions $G_{\mu_{X}}$ for the hyperbolic metrics on the modular curves $X$. We get a hyperbolic Hodge correlator map on the modular curves 11

$$
\operatorname{Cor}_{\mathcal{H}, \mu_{X}}^{h}: \mathcal{C T}\left(\mathbb{V}_{X, S}\right) \longrightarrow H^{2}(X) \text {. }
$$

The integral over the fundamental cycle of $X$ provides an isomorphism $H^{2}(X) \rightarrow \mathbb{C}$. So we may assume that for an individual curve $X$ the correlator is a complex number. However moving from $X$ to $Y$ we multiply it by $\operatorname{deg} \pi$.

Proposition 12.2. The hyperbolic Hodge correlator maps are compatible with natural projections $\pi: Y \rightarrow X$ of modular curves:

$$
\operatorname{Cor}_{\mathcal{H}, \mu_{Y}}^{h} \mathcal{C}\left(\pi^{*} v_{1} \otimes \ldots \otimes \pi^{*} v_{m}\right)=\pi^{*} \operatorname{Cor}_{\mathcal{H}, \mu_{X}}^{h} \mathcal{C}\left(v_{1} \otimes \ldots \otimes v_{m}\right) \in H^{2}(Y), \quad v_{i} \in \mathbb{V}_{X, S}
$$

Proof. Lemma 12.1 plus the projection formula implies that, given 1-forms $\omega_{i}$ on $X$, we have

$$
\int_{Y} \pi^{*} \omega_{1} \wedge \pi^{*} \omega_{2} \cdot G_{\mu_{Y}}\left(y_{1}, y_{2}\right)=\int_{X} \omega_{1} \wedge \omega_{2} \cdot \pi_{1 *} G_{\mu_{Y}}\left(x_{1}, y_{2}\right)=\int_{X} \omega_{1} \wedge \omega_{2} \cdot \pi_{2}^{*} G_{\mu_{X}}\left(x_{1}, y_{2}\right) .
$$

Here the integrals are over $y_{1}$ and $x_{1}$. Similarly, we have

$$
G_{\mu_{Y}}\left(\pi^{*}\{s\}, y_{2}\right)=\pi_{2}^{*} G_{\mu_{X}}\left(s, x_{2}\right) .
$$

Therefore we have an identity similar to (278) where one or two of the decorating forms $\omega_{i}$ 's are replaced by the decorating divisors $\pi^{*}\left\{s_{i}\right\}$ - we understood (278) as the Hodge correlator for a tree with one internal trivalent vertex.

Let us use the induction on $m$. We understood the correlators are numbers via the isomorphism $H^{2}(X) \stackrel{\sim}{\rightarrow} \mathbb{C}$. The $m=3$ case follows from (279), using

$$
\int_{Y} \pi^{*} \eta_{1} \wedge \pi^{*} \eta_{2} \cdot \pi^{*} \varphi_{3}=\operatorname{deg} \pi \int_{X} \eta_{1} \wedge \eta_{2} \cdot \varphi_{3}
$$

For the induction step, take a plane trivalent tree $T$ decorated by $\pi^{*} v_{1} \otimes \ldots \otimes \pi^{*} v_{m}$. Take two external edges sharing an internal vertex $w$ and decorated by $\pi^{*} v_{i}$ and $\pi^{*} v_{i+1}$. Applying (278) to the integral over the curve $Y$ corresponding to $w$, we reduce the claim to the case of a tree with $m-1$ external vertices.

Let $\mathcal{M}$ be the universal modular curve, that is the projective limit of the tower of modular curves. It is defined over $\overline{\mathbb{Q}}$. Since Hecke operators split the Grothendieck motive $H^{1}(\mathcal{M})$ into a direct sum of the cuspidal and Eisenstein parts, $\operatorname{gr}^{W} H^{1}(\mathcal{M}) \otimes \overline{\mathbb{Q}}=H^{1}(\mathcal{M}) \otimes \overline{\mathbb{Q}}$. So one has

$$
\mathbb{H}_{\mathcal{M}}^{\vee}=H^{1}(\mathcal{M}) \otimes \overline{\mathbb{Q}}=\bigoplus_{M} M^{\vee} \otimes V_{M} \bigoplus \mathbb{Q}(\widehat{\text { Cusps }})_{0}(-1)
$$

Here the first sum is over all pure rank 2 , weight -1 motives $M$ over $\mathbb{Q}$. The subscript 0 in the second summand is used to denote the subspace of measures functions with zero mass. The $\overline{\mathbb{Q}}$-vector space $V_{M}$ is a representation of the finite adele group $G L_{2}\left(\mathbb{A}_{\mathbb{Q}}^{f}\right)$. The tensor product of

\footnotetext{
${ }^{11}$ Hyperbolic Hodge correlators are a special case of the Hodge correlators, specified by the choice of the metric.
} 
the Steinberg representation of $G L_{2}(\mathbb{R})$ and $V_{M}$ is an automorphic representation of $G L_{2}\left(\mathbb{A}_{\mathbb{Q}}\right)$ corresponding to the pure motive $M$ via the Langlands correspondence.

In the de Rham realization, we get an adelic description of $H_{D R}^{1}(\mathcal{M}, \mathbb{C})$ :

$$
H_{D R}^{1}(\mathcal{M}, \mathbb{C})=\bigoplus_{M}\left(\Omega_{M}^{1} \oplus \bar{\Omega}_{M}^{1}\right) \otimes V_{M} \bigoplus \mathbb{Q}(\widehat{\operatorname{Cusps}})_{0}(-1)
$$

Here $\Omega_{M}^{1}$ is the (1,0)-part of the de Rham realization of the motive $M$. Proposition 12.2 implies that the hyperbolic Hodge correlator map provides a $G L_{2}\left(\mathbb{A}_{\mathbb{Q}}^{f}\right)$-covariant map

$$
\operatorname{Cor}_{\mathcal{H}}^{h}: \mathcal{C T}\left(H_{D R}^{1}(\mathcal{M}, \mathbb{C})\right)(1) \rightarrow \mathbb{C} .
$$

Indeed, it is $G L_{2}(\widehat{\mathbb{Z}})$-covariant on the nose, and $G L_{2}(\mathbb{Q})$-covariant thanks to the compatibility with the projections established in Proposition 12.2. Since these two groups generate $G L_{2}\left(\mathbb{A}_{\mathbb{Q}}^{f}\right)$, it descends to a map of $G L_{2}\left(\mathbb{A}_{\mathbb{Q}}^{f}\right)$-coinvariants

$$
\operatorname{Cor}_{\mathcal{H}}^{h}: \mathcal{C T}\left(H_{D R}^{1}(\mathcal{M}, \mathbb{C})\right)(1)_{G L_{2}\left(\mathbb{A}_{\mathbb{Q}}^{f}\right)} \longrightarrow \mathbb{C} .
$$

The map (281) is a generalization of the Rankin-Selberg integrals: the latter appear for the triple tensor product of copies of $H_{D R}^{1}(\mathcal{M}, \mathbb{C})$, as explained in 12.1.1. Restricting this map to the subspace $\overline{\mathcal{C} \mathcal{L} i e} \vee\left(H_{D R}^{1}(\mathcal{M}, \mathbb{C})\right)$ of the cyclic tensor product of $H_{D R}^{1}(\mathcal{M}, \mathbb{C})$ (Section 10.4) we get the periods of $\pi_{1}^{\text {nil }}(\mathcal{M})$.

\subsection{Motivic correlators in towers}

Let $\pi: X^{\prime} \rightarrow X$ be a nontrivial map of projective curves, $S^{\prime}$ and $S$ are subsets of points of $X^{\prime}$ and $X$, and $\pi\left(S^{\prime}\right)=S$. We say that vectors $v_{s^{\prime}}$ and $v_{s}$ at the points $s^{\prime} \in S^{\prime}$ and $s \in S$ are compatible with respect to the map $\pi$ if for local parameters $t^{\prime}$ and $t$ such that $d t^{\prime}$ and $d t$ are dual to the vectors $v^{\prime}$ and $v$ the restriction of $\left(t^{\prime}\right)^{m_{\pi}\left(s^{\prime}\right)} / \pi^{*} t$ to $s^{\prime}$ equals 1 , where $m_{\pi}\left(s^{\prime}\right)$ is the multiplicity of the map $\pi$ at the point $s^{\prime}$. In particular, if $m_{\pi}\left(s^{\prime}\right)=1$, this means that $d \pi_{*}\left(v^{\prime}\right)=v$. Let us assume that for each $s \in S$ (respectively $s^{\prime} \in S^{\prime}$ ) there is a non-zero vector $v_{s} \in T_{s} X$ (respectively $v_{s^{\prime}} \in T_{s^{\prime}} X$ ), and that these vectors are compatible under the map $\pi$. Denote by $\operatorname{deg}(\pi)$ the degree of the map $\pi$. Recall

$$
\mathbb{V}_{X, S}^{\vee}:=H^{1}(X) \oplus \mathbb{Q}[S](-1) ; \quad \mathbb{V}_{X, S^{*}}^{\vee} \cong \operatorname{gr}^{W} H^{1}(X-S)
$$

There are maps

$$
\pi^{*}: \mathbb{V}_{X, S}^{\vee} \longrightarrow \mathbb{V}_{X^{\prime}, S^{\prime}}^{\vee}, \quad \pi_{*}: \mathbb{V}_{X^{\prime}, S^{\prime}}^{\vee} \longrightarrow \mathbb{V}_{X, S}^{\vee}, \quad \pi_{*} \pi^{*}=\operatorname{deg}(\pi) .
$$

The restriction of the map $\pi^{*}$ to $\mathbb{Q}[S](-1)$ is given by the following map:

$$
\pi_{S}^{*}: \mathbb{Q}[S] \rightarrow \mathbb{Q}\left[S^{\prime}\right] ; \quad\{s\} \rightarrow \sum_{s^{\prime} \in \pi^{-1}(s)} m_{\pi}\left(s^{\prime}\right)\left\{s^{\prime}\right\}
$$

So $\operatorname{deg} \pi_{S}^{*}(\{s\})=\operatorname{deg} \pi$. The maps $\pi^{*}$ and $\pi_{S}^{*}$ induce a map of vector spaces

$$
\pi_{\otimes}^{*}: \mathcal{C} \mathrm{T}\left(\mathbb{V}_{X, S}^{\vee}\right) \otimes \mathbb{Q}[S] \longrightarrow \mathcal{C} \mathrm{T}\left(\mathbb{V}_{X^{\prime}, S^{\prime}}^{\vee}\right) \otimes \mathbb{Q}\left[S^{\prime}\right] .
$$

Motivic correlators require a choice of a tangential base point. One can consider the motivic correlator map obtained by using an arbitrary tangential base point. Then its source is the space $\mathcal{C} T\left(\mathbb{V}_{X, S}^{\vee}\right) \otimes \mathbb{Q}[S]$, where the second factor $\mathbb{Q}[S]$ bookkeeps the choice of the point $s \in S$ where we put the tangential base point. 
Lemma 12.3. Assume that the tangential base points at $S^{\prime}$ and $S$ are compatible with the map $\pi: X^{\prime}-S^{\prime} \rightarrow X-S$. Then there is a commutative diagram, with the horizontal rows provided by (246), and the vertical maps are induced by the map $\pi^{*}$ :

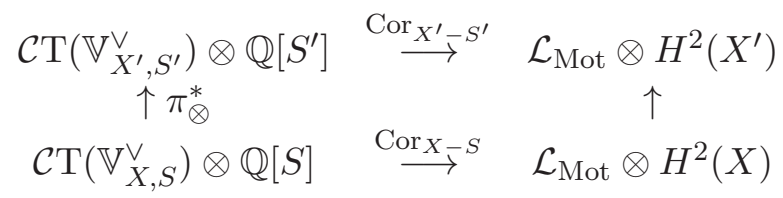

Proof. If $\pi$ is unramified at every point of $S^{\prime}$ it follows straight from the definition. The general case is deduced from this, say, by the specialization to a ramified point.

Let $\operatorname{tr}_{X}: H^{2}(X) \rightarrow \mathbb{Q}(-1)$ be the canonical map. Since $\operatorname{tr}_{X^{\prime}} \pi^{*}=\operatorname{deg}(\pi) \operatorname{tr}_{X}$, we get a commutative diagram

$$
\begin{array}{rcc}
\mathcal{C} \mathrm{T}\left(\mathbb{V}_{X^{\prime}, S^{\prime}}^{\vee}\right) \otimes \mathbb{Q}\left[S^{\prime}\right](1) & \stackrel{\mathrm{Cor}_{X^{\prime}-S^{\prime}}}{\longrightarrow} & \mathcal{L}_{\mathrm{Mot}} \\
\uparrow \pi^{*} \otimes \operatorname{deg}(\pi)^{-1} \pi_{\otimes}^{*} & \uparrow= \\
\mathcal{C} \mathrm{T}\left(\mathbb{V}_{X, S}^{\vee}\right) \otimes \mathbb{Q}[S](1) & \stackrel{\mathrm{Cor}_{X}-S}{\longrightarrow} & \mathcal{L}_{\mathrm{Mot}}
\end{array}
$$

Remark. Denote by $\pi_{\mathcal{C}}^{*}$ the left vertical map in (283). It is not a map of Lie coalgebras. However it will become a Lie coalgebra map if we consider the quotient of the Lie coalgebra $\mathcal{C} \mathrm{T}\left(\mathbb{V}_{X^{\prime}, S^{\prime}}^{\vee}\right)(1)$ defined in Definition 12.4 .

Now suppose that we have a projective system $\left(X_{\alpha}, S_{\alpha}\right)$ of curves $X_{\alpha}$ with finite subsets of points $S_{\alpha}$ on them and maps between them as above. Using the normalized maps $\operatorname{deg}(\pi)^{-1} \pi_{\otimes}^{*}$, functions on the sets $S_{\alpha}$ in the limit turn into measures on the projective limit $\widehat{S}:=\lim S_{\alpha}$. We denote the space of measures by $\operatorname{Meas}(\widehat{S})$. So using (283), we get a map of the corresponding inductive limit

$$
\underset{\lim }{\longrightarrow} \mathcal{C}\left(\mathbb{V}_{X_{\alpha}, S_{\alpha}}^{\vee}\right) \otimes \operatorname{Meas}(\widehat{S})(1) \longrightarrow \mathcal{L}_{\mathrm{Mot}}
$$

Here $\mathcal{X}$ denotes the projective limit of the curves $X_{\alpha}-S_{\alpha}$. Abusing notation, we denote below the inductive limit in (284) by $\mathcal{C} T\left(\mathbb{V}_{\mathcal{X}}^{\vee}\right) \otimes \operatorname{Meas}(\widehat{S})_{0}(1)$.

Examples. 1. Let $E$ be an elliptic curve. The curves $E-E[N]$ and the isogenies $E-$ $E[M N] \rightarrow E-E[N]$ form a tower. We assign to every missing point on $E-E[N]$ the tangent vector $\frac{1}{N} v_{\Delta}$. We get a compatible family of tangential base points for the tower $\{E-E[N]\}$. Denote by $\widehat{E_{\mathrm{tors}}}$ the Tate module of $E$, that is the projective limit of the torsion groups of $E$. Therefore we arrive at the motivic correlator map, where the subscript $\widehat{E_{\text {tors }}}$ refers to the coinvariants for the action of the group $\widehat{E_{\text {tors }}}$ :

$$
\operatorname{Cor}_{\mathcal{E}}:\left(\mathcal{C T}\left(\mathbb{V}_{\mathcal{E}}^{\vee}\right) \otimes \operatorname{Meas}\left(\widehat{E_{\text {tors }}}(1)\right)\right)_{\widehat{E_{\text {tors }}}} \longrightarrow \mathcal{L}_{\text {Mot }}
$$

There is a unique $\widehat{E_{\mathrm{tors}}}-$ invariant measure $\mu_{\mathcal{E}}^{0}$ on the Tate module $\widehat{E_{\mathrm{tors}}}$. So we get a map

$$
\operatorname{Cor}_{\mathcal{E}}^{0}: \mathcal{C} \mathrm{T}\left(\mathbb{V}_{\mathcal{E}}^{\vee}\right)(1) \otimes \mu_{\mathcal{E}}^{0} \longrightarrow \mathcal{L}_{\text {Mot }}
$$

2. Similarly, when $X=\mathbb{P}^{1}$ and $S=\{0\} \cup\{\infty\} \cup \mu_{l^{\infty}}$ we get the measures on $\mathbb{Z}_{l}(1)$. 
Definition 12.4. $\mathcal{C}^{\prime} \mathrm{T}\left(\mathbb{V}_{X^{\prime}, S^{\prime}}^{\vee}\right)$ is the quotient of $\mathcal{C} \mathrm{T}\left(\mathbb{V}_{X^{\prime}, S^{\prime}}^{\vee}\right)$ by the subobject spanned by expressions

$$
\mathcal{C}\left(\pi^{*} v_{1} \otimes \ldots \otimes \pi^{*} v_{m-1} \otimes v_{m}\right), \quad v_{1}, \ldots, v_{m-1} \in \mathbb{V}_{X, S}^{\vee}, \quad v_{m} \in \operatorname{Ker} \pi_{*} \subset \mathbb{V}_{X^{\prime}, S^{\prime}}^{\vee}
$$

Lemma 12.5. The correlator map $\operatorname{Cor}_{X-S}$ annihilates elements

$$
\mathcal{C}\left(\pi^{*} v_{1} \otimes \ldots \otimes \pi^{*} v_{m-1} \otimes v_{m}\right) \otimes \pi_{S}^{*}\{s\}(1) \text { such that } \pi_{*} v_{m}=0 .
$$

Proof. We prove this in the $l$-adic realization. A proof for another realizations is similar. It is sufficient to prove the case when $\pi: X^{\prime}-S^{\prime} \rightarrow X-S$ is a Galois cover with a group $G$. The action of $\operatorname{Gal}(\bar{F} / F)$ commutes with the action of $G$. Thus

$$
\begin{aligned}
& \operatorname{Cor}_{X^{\prime}-S^{\prime}} \mathcal{C}\left(\pi^{*} v_{1} \otimes \ldots \otimes \pi^{*} v_{m-1} \otimes v_{m}\right) \otimes \pi_{S}^{*}\{s\}= \\
& \operatorname{Cor}_{X^{\prime}-S^{\prime}} \frac{1}{|G|} \sum_{g \in G} g^{*} \mathcal{C}\left(\pi^{*} v_{1} \otimes \ldots \otimes \pi^{*} v_{m-1} \otimes v_{m}\right) \otimes \pi_{S}^{*}\{s\}= \\
& \operatorname{Cor}_{X^{\prime}-S^{\prime}} \mathcal{C}\left(\pi^{*} v_{1} \otimes \ldots \otimes \pi^{*} v_{m-1} \otimes \pi_{*} v_{m}\right) \otimes \pi_{S}^{*}\{s\}=0 .
\end{aligned}
$$

Here is an analytic counterpart:

Lemma 12.6. Let $\pi: X^{\prime} \rightarrow X$ and $\pi_{*}\left(\mu_{X^{\prime}}\right)=\mu_{X}$. Then

$$
\operatorname{Cor}_{\mathcal{H}, \mu_{X^{\prime}}} \mathcal{C}\left(\pi^{*} v_{1} \otimes \ldots \otimes \pi^{*} v_{m-1} \otimes v_{m}\right)=0 \text { for } m \geq 3 \text { and } \pi_{*} v_{m}=0 .
$$

Proof. We prove it by the induction on $m$. The $m=3$ case is clear from (278). Take a plane trivalent tree $T$ decorated by $\pi^{*} v_{1} \otimes \ldots \otimes \pi^{*} v_{m-1} \otimes v_{m}$. Take two external edges sharing an internal vertex $w$ and decorated by $\pi^{*} v_{i}$ and $\pi^{*} v_{i+1}$. Using (278) for the integral over the curve assigned to $w$ we reduce the claim to the case of a tree with $m-1$ external vertices.

Lemma 12.7. $\mathcal{C}^{\prime} \mathrm{T}\left(\mathbb{V}_{X^{\prime}, S^{\prime}}^{\vee}\right)(1)$ inherits a Lie coalgebra structure; $\pi_{\mathcal{C}}^{*}$ is a Lie coalgebra map.

Proof. Let us check that elements (285) span a coideal for the cobracket $\delta=\delta_{\text {Cas }}+\delta_{S}$. A typical term of $\delta_{\text {Cas }}(285)$ is $\sum_{k} \mathcal{C}\left(M_{1} \otimes \alpha_{k}\right) \wedge \mathcal{C}\left(\alpha_{k}^{\vee} \otimes M_{2}\right)$, where $\sum_{k} \alpha_{k} \otimes \alpha_{k}^{\vee}$ is the Casimir element in $H^{1}\left(X^{\prime}\right) \otimes H^{1}\left(X^{\prime}\right)$, and $\mathcal{C}\left(M_{1} \otimes M_{2}\right)$ is the element (285) $)$. There is a decomposition

$$
H^{1}\left(X^{\prime}\right)=\pi^{*} H^{1}(X) \oplus \pi^{*} H^{1}(X)^{\perp} .
$$

The summands are orthogonal for the intersection pairing on $H^{1}\left(X^{\prime}\right)$. The map $\pi_{*}$ annihilates the second summand. We may assume that $v_{m}$ enters $M_{2}$. If $\alpha_{k} \in \pi^{*} H^{1}(X)$, then $\alpha_{k}^{\vee} \in$ $\pi^{*} H^{1}(X)$, and $\mathcal{C}\left(\alpha_{k}^{\vee} \otimes M_{2}\right)$ is of type (285). Otherwise $\alpha_{k}, \alpha_{k}^{\vee} \in \pi^{*} H^{1}(X)^{\perp}$, thus $\mathcal{C}\left(\alpha_{k} \otimes M_{1}\right)$ is of type (285). The $\delta_{S}$ term deals with the $S$-decorated vertices. Its typical term is

$\sum_{i} \mathcal{C}\left(M_{1} \otimes s_{i}\right) \wedge \mathcal{C}\left(s_{i} \otimes M_{2}\right)=\sum_{i} \mathcal{C}\left(M_{1} \otimes\left(s_{i}-s_{1}\right)\right) \wedge \mathcal{C}\left(s_{i} \otimes M_{2}\right)+\mathcal{C}\left(M_{1} \otimes s_{1}\right) \wedge \mathcal{C}\left(\sum_{i} s_{i} \otimes M_{2}\right)$.

The left factor of the first summand, and the right factor of the second are of type (285).

Corollary 12.8. Set $\mu_{S}:=\frac{1}{|S|} \sum_{s \in S}\{s\}$. The map $\operatorname{Cor}_{X-S}$ gives rise to a Lie coalgebra map.

$$
\operatorname{Cor}_{X-S}^{\mathrm{av}}: \mathcal{C}^{\prime} \mathrm{T}\left(\mathbb{V}_{X, S}^{\vee}\right) \otimes \mu_{S} \otimes H_{2}(X) \longrightarrow \mathcal{L}_{\text {Mot }}
$$

Proof. Follows from Lemmas 12.5 and 12.7. 


\subsection{Motivic correlators for the universal modular curve}

Recall the universal modular curve $\mathcal{M}$ and decomposition (280). The tower of modular curves has a natural family of tangential base points at the cusps provided by exponents of the canonical parameter $z$ on the upper half plane. Precisely, if the stabilizer of a cusp is an index $N$ subgroup of the maximal unipotent subgroup in $S L_{2}(\mathbb{Z})$, it is $\exp (2 \pi i z / N)$. They are compatible, in the sense of Section 12.2, with the natural projections of the modular curves. Going to the limit in the tower of modular curves, as explained in Section 12.2, we arrive at the following picture. There is a Lie coalgebra in the $\otimes$-category of pure motives generated by $H^{1}(\overline{\mathcal{M}})$ :

$$
\mathcal{C}^{\prime} \mathrm{T}\left(\mathbb{V}_{\mathcal{M}}^{\vee}\right) \otimes \operatorname{Meas}(\widehat{\operatorname{Cusps}})_{0}(1)
$$

The group $G L_{2}(\widehat{\mathbb{Z}})$ acts by its automorphisms. The group $G L_{2}(\mathbb{Q})$ acts by its automorphisms thanks to the compatibility with projections (Section 12.2). So the group $G L_{2}\left(\mathbb{A}_{\mathbb{Q}}^{f}\right)$ acts. Moreover, the canonical map of Lie coalgebras

$$
\operatorname{Cor}_{\mathcal{M}}: \mathcal{C}^{\prime} \mathrm{T}\left(\mathbb{V}_{\mathcal{M}}^{\vee}\right) \otimes \operatorname{Meas}(\widehat{\operatorname{Cusps}})_{0}(1) \longrightarrow \mathcal{L}_{\text {Mot }}
$$

is $G L_{2}\left(\mathbb{A}_{\mathbb{Q}}^{f}\right)$-equivariant. (The group acts trivially on the right). So it descends to a map of the coinvariants

$$
\operatorname{Cor}_{\mathcal{M}}:\left(\mathcal{C}^{\prime} \mathrm{T}\left(\mathbb{V}_{\mathcal{M}}^{\vee}\right) \otimes \operatorname{Meas}(\widehat{\operatorname{Cusps}})_{0}(1)\right)_{G L_{2}\left(\mathbb{A}_{\mathbb{Q}}^{f}\right)} \longrightarrow \mathcal{L}_{\mathrm{Mot}}
$$

In the de Rham realization there is a commutative diagram

$$
\left(\mathcal{C}^{\prime} \mathrm{T}\left(H_{D R}^{1}(\mathcal{M})\right)\right) \otimes \operatorname{Meas}\left(\widehat{\operatorname{Cusps}}_{0}(1)\right)_{G L_{2}\left(\mathbb{A}_{\mathbb{Q}}^{f}\right)} \stackrel{\operatorname{Cor}_{\mathcal{M}}}{\longrightarrow} \mathcal{L}_{\text {Mot }}
$$

where the diagonal arrow is the Hodge correlator map, and the vertical one is the period map.

Lemma 12.9. There is a unique $G L_{2}\left(\mathbb{A}_{\mathbb{Q}}^{f}\right)$-covariant volume 1 measure $\mu^{0} \in \operatorname{Meas}(\widehat{\operatorname{Cusps}})$,

Proof. Let $U$ be the upper triangular unipotent subgroup of $G L_{2}$ and $B$ the normalizing it Borel subgroup. Then there are isomorphisms

$$
\widehat{\operatorname{Cusps}}=G L_{2}(\widehat{\mathbb{Z}}) / U(\widehat{\mathbb{Z}})=G L_{2}\left(\mathbb{A}_{\mathbb{Q}}^{f}\right) / B(\mathbb{Q}) U\left(\mathbb{A}_{\mathbb{Q}}^{f}\right)
$$

The first is obvious since the cusps on the modular curve $Y(N)$ are identified with the coset $G L_{2}(\mathbb{Z} / N \mathbb{Z}) / U(\mathbb{Z} / N \mathbb{Z})$, the second follows from the decomposition $G L_{2}\left(\mathbb{A}_{\mathbb{Q}}^{f}\right)=B(\mathbb{Q}) G L_{2}(\widehat{\mathbb{Z}})$. A Haar measure on $G L_{2}\left(\mathbb{A}_{\mathbb{Q}}^{f}\right)$ induces a covariant measure on the quotient. We normalize it by the volume one condition.

Taking the covariant measure $\mu^{0}$ we are getting a map

$$
\operatorname{Cor}_{\mathcal{M}}^{0}: \mathcal{C}^{\prime} \mathrm{T}\left(\mathbb{V}_{\mathcal{M}}^{\vee}\right)(1)_{G L_{2}\left(\mathbb{A}_{\mathbb{Q}}^{f}\right)} \otimes \mu^{0} \longrightarrow \mathcal{L}_{\text {Mot }}
$$


Lemma 12.10. The motivic correlators for the classical Rankin-Selberg integrals (Section [12.1) are motivic Ext $^{1}$ 's:

$$
\begin{gathered}
\operatorname{Cor}_{\mathcal{M}}^{0} \mathcal{C}\left(\left\{a_{0}\right\} \otimes\left\{a_{1}\right\} \otimes M_{\omega}^{\vee}\right) \in \operatorname{Ext}_{\text {Mot }}^{1}\left(\mathbb{Q}(0), M_{\omega}(1)\right) . \\
\operatorname{Cor}_{\mathcal{M}}^{0} \mathcal{C}\left(\left\{a_{0}\right\} \otimes M_{\omega_{1}}^{\vee} \otimes M_{\omega_{2}}^{\vee}\right) \in \operatorname{Ext}_{\operatorname{Mot}}^{1}\left(\mathbb{Q}(0), M_{\omega_{1}} \otimes M_{\omega_{2}}\right) .
\end{gathered}
$$

Proof. The first and the second motivic correlators on the left live, respectively, in the $M_{\omega}(1)$ isotypical component, and $M_{\omega_{1}} \otimes M_{\omega_{2}}$-isotypical component of the motivic Lie coalgebra. The first defines an element of $\operatorname{Ext}_{\text {Mot }}^{1}\left(\mathbb{Q}(0), M_{\omega}(1)\right)$ if and only if its coproduct there is zero. The second defines an element of $\operatorname{Ext}_{\text {Mot }}^{1}\left(\mathbb{Q}(0), M_{\omega}(1) \otimes M_{\omega_{2}}\right)$ if and only if its coproduct is zero.

Let us compute the second coproduct. Set $\mathbb{V}:=\mathbb{V} \overline{\mathcal{M}}$.

$$
\delta \operatorname{Cor}_{\mathcal{M}}^{0} \mathcal{C}\left(\left\{a_{0}\right\} \otimes M_{\omega_{1}}^{\vee} \otimes M_{\omega_{2}}^{\vee}\right)=\operatorname{Cor}_{\mathcal{M}}^{0} \mathcal{C}\left(\left\{a_{0}\right\} \otimes \mathbb{V}\right) \wedge \operatorname{Cor}_{\mathcal{M}}^{0} \mathcal{C}\left(\mathbb{H} \otimes M_{\omega_{1}}^{\vee} \otimes M_{\omega_{2}}^{\vee}\right)
$$

One has $\operatorname{Cor}_{\mathcal{M}} \mathcal{C}\left(\left\{a_{0}\right\} \otimes \mathbb{V}\right)(1) \otimes\{s\}=a_{0}-s \in J_{X} \otimes \mathbb{Q}=\operatorname{Ext}_{\operatorname{Mot}}^{1}(\mathbb{Q}(0), \mathbb{V})$. Since $s, a_{0}$ are cusps, it is zero by the Manin-Drinfeld theorem. Thus $\operatorname{Cor}_{\mathcal{M}}^{0} \mathcal{C}\left(\left\{a_{0}\right\} \otimes \mathbb{V}\right)(1)=0$, and hence the coproduct is zero. This implies the second claim. The proof of the first claim is similar.

For certain divisors $s_{i}$ these elements are not zero, and hypothetically generate the Ext ${ }^{1}$ groups.

How to describe the image of the motivic correlator map (287)? Recall the hypothetical abelian category $\mathcal{C}_{\mathcal{M}}$ of mixed motives generated by $H^{1}(\mathcal{M})$. Let $\mathcal{P}_{\mathcal{M}}$ be the semi-simple category of pure motives over $\overline{\mathbb{Q}}$ generated by $H^{1}(\mathcal{M})$. Since $\overline{\mathbb{Q}}$ is of arithmetic dimension 1 , the motivic Lie algebra of the mixed category $\mathcal{C}_{\mathcal{M}}$ is conjectured to be a free Lie algebra in the category $\mathcal{P}_{\mathcal{M}}$. Let $\mathcal{L}_{\mathcal{M}}$ be the dual motivic Lie coalgebra. The map (287) lands in $\mathcal{L}_{\mathcal{M}}$ :

$$
\operatorname{Cor}_{\mathcal{M}}^{0}: \mathcal{C}^{\prime} \mathrm{T}\left(\mathbb{V}_{\mathcal{M}}^{\vee}\right)(1)_{G L_{2}\left(\mathbb{A}_{\mathbb{Q}}^{f}\right)} \otimes \mu^{0} \longrightarrow \mathcal{L}_{\mathcal{M}}
$$

The space of its cogenerators of type $N^{\vee}$, where $N$ is a pure motive of negative weight from $\mathcal{P}_{\mathcal{M}}$, should be isomorphic to $\operatorname{Ext}^{1}(\mathbb{Q}(0), N) \otimes N^{\vee}$; Beilinson's conjectures predict its dimension.

The map (288) is not surjective for a very simple reason. The motivic correlators do not give non-trivial elements of the Jacobian $J_{\mathcal{M}} \otimes \mathbb{Q}$ of the universal modular curve $\mathcal{M}$. Indeed, we get in the image of (288) only the images of degree zero divisors supported at the cusps (Section 10,4.1), which are zero by the Manin-Drinfeld theorem. On the other hand the simplest component of $\mathcal{L}_{\mathcal{M}}$ is given by $\operatorname{Ext}^{1}\left(\mathbb{Q}(0), H_{1}(\mathcal{M})\right)=J_{\mathcal{M}} \otimes \mathbb{Q}$. However Lemma 12.10 and the Rankin-Selberg method suggest that we do get $\operatorname{Ext}^{1}(\mathbb{Q}(0), N)$ for $N=H_{1}(\mathcal{M})(1)$ and $N=H_{1}(\mathcal{M}) \otimes H_{1}(\mathcal{M})$.

More specifically, let $V_{\omega_{i}}$ (resp. $\left.M_{\omega_{i}}\right)$ be the representation of $G L_{2}\left(\mathbb{A}_{\mathbb{Q}}^{f}\right)$ (resp. the weight -1 motive) corresponding to a Hecke eigenform $\omega_{i}$. It follows from (288) that the motivic correlator provides a map

$$
\operatorname{Cor}_{\mathcal{M}}^{0}: \mathcal{C}^{\prime}\left(\bigotimes_{i=1}^{k} M_{\omega_{i}}^{\vee} \otimes V_{\omega_{i}}\right)(1) \longrightarrow \mathcal{L}_{\otimes_{i=1}^{k} M_{\omega_{i}}(-1)} \bigotimes \otimes_{i=1}^{k} M_{\omega_{i}}^{\vee}(1)
$$

It is essentially described by a map of vector spaces $\mathcal{C}^{\prime}\left(\bigotimes_{i=1}^{k} V_{\omega_{i}}\right) \longrightarrow \mathcal{L}_{\otimes_{i=1}^{k} M_{\omega_{i}}(-1)}$. 
Examples. 1. Consider the motivic correlator corresponding to the tensor product of the motives of Hecke eigenforms $\omega_{1}, \ldots \omega_{4}$ as on Fig 34, we conjecture that there are vectors $v_{\omega_{i}} \in V_{\omega_{i}}$ providing non-zero elements

$$
\operatorname{Cor}_{\mathcal{M}}^{0} \mathcal{C}\left(\bigotimes_{i=1}^{4} M_{\omega_{i}}^{\vee} \otimes v_{\omega_{i}}\right)(1) \in \operatorname{Ext}_{\mathcal{M M}}^{1}\left(\mathbb{Q}(0), \otimes_{i=1}^{4} M_{\omega_{i}}(-1)\right)
$$

2. As discussed in Section 1.10, there are two types of cyclic elements, see (53) and Fig 36, which should be related to $L\left(S^{2} M_{\omega}, 3\right)$. Let us consider first the motivic correlator map assigned to the left one:

$$
\operatorname{Cor}_{\mathcal{M}}^{0}: \mathcal{C}\left(\operatorname{Meas}(\widehat{\operatorname{Cusps}})(-1)^{\otimes 2} \otimes S^{2}\left(M_{\omega}^{\vee} \otimes V_{\omega}\right)(1)\right) \longrightarrow \mathcal{L}_{\mathcal{M}}
$$

By the Manin-Drinfeld theorem the components of the coproduct corresponding to the $s$ decorated vertices are zero. Furthermore, the only nontrivial component of the coproduct is the one corresponding to the cut shown on Fig 36 by a dotted line. It is given by

$$
\sum_{i} \operatorname{Cor}_{\mathcal{M}}^{0}\left(s_{1} \otimes \omega_{1} \otimes \psi_{i}(1)\right) \otimes \operatorname{Cor}_{\mathcal{M}}^{0}\left(\psi_{i}^{\vee} \otimes \omega_{2} \otimes s_{2}(1)\right)
$$

To formulate a criteria for this element to be zero, we proceed as follows:

i) Since $s_{i}$ are cuspidal divisors on a modular curve, each of the factors determines an element of $\operatorname{Ext}_{\text {Mot }}^{1}$, e.g. $\operatorname{Cor}_{\mathcal{M}}^{0}\left(s_{1} \otimes \omega_{1} \otimes \psi_{i}(1)\right) \in \operatorname{Ext}_{\text {Mot }}^{1}\left(\mathbb{Q}(0), M_{\omega_{1}} \otimes M_{\psi_{i}}\right)$.

ii) We calculate the corresponding tensor product of the Hodge correlators, given explicitly by the Rankin-Selberg integrals:

$$
\sum_{i} \operatorname{Cor}_{\mathcal{H}}^{0}\left(s_{1} \otimes \omega_{1} \otimes \psi_{i}(1)\right) \otimes \operatorname{Cor}_{\mathcal{H}}^{0}\left(\psi_{i}^{\vee} \otimes \omega_{2} \otimes s_{2}(1)\right) \in \mathbb{C} \otimes \mathbb{C} .
$$

The conjectural injectivity of the regulator map implies that (291) vanishes if and only if the coproduct (290) is zero.

The cyclic element provided by the right diagram on Fig 36 is treated similarly. This completes the formulation of Conjecture 1.16 relating $L\left(\operatorname{Sym}^{2} f, 3\right)$ to the Hodge correlators.
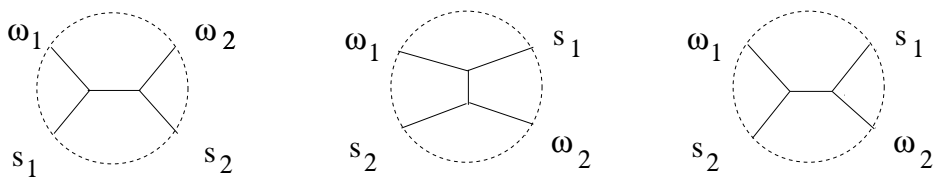

Figure 36: Correlator integrals of type $S^{2} M_{\omega}(1)$.

3. It would be interesting to generalize this picture, providing an automorphic description of a part of the motivic Galois group of the category of mixed motives over an arithmetic field $F$. Here is an example provided by the Drinfeld modular curves Dr2] in the function field case.

$l$-adic correlators for the universal Drinfeld modular curve. Let $F$ be the function field of a curve $X$ over a finite field. Choose a point $x$ on $X$ and take the Drinfeld modular curve parametrising the rank 2 elliptic modules corresponding to the affine curve $X-x$ [Dr2]. 
Then there is the same kind of picture as above. Namely, one should have a conjectural abelian category of mixed motives over $F$. One can define its $l$-adic version: a category of motivic $l$-adic Galois representations of the Galois group $\operatorname{Gal}(\bar{F} / F)$, where $l \neq \operatorname{char}(F)$. An example is provided by the $l$-adic fundamental Lie algebra of the Drinfeld modular curve. It is a free pronilpotent Lie algebra generated by $H_{1}$ of the curve. There is a decomposition similar to (280). Thus, just as above, we arrive at the $l$-adic correlator map.

\section{Feynman integral for the Hodge correlators}

\subsection{The Feynman integral}

Let $\varphi$ be a smooth function on a complex curve $X(\mathbb{C})$ with values in $N \times N$ complex matrices $\operatorname{Mat}_{N}(\mathbb{C})$. We say that $\{\varphi\}$ is our space of fields. Let us choose a cyclic word

$$
W=\mathcal{C}\left(\left\{a_{0}\right\} \otimes \omega_{1}^{0} \otimes \ldots \otimes \omega_{n_{0}}^{0} \otimes \ldots \otimes\left\{a_{m}\right\} \otimes \omega_{1}^{m} \otimes \ldots \otimes \omega_{n_{m}}^{m}\right) \in \mathcal{C} \mathrm{T}\left(\mathrm{V}_{X, S}^{\vee}\right)
$$

where $a_{i}$ are points of $X(\mathbb{C})$, not necessarily different, and $\omega_{j}^{i} \in \Omega_{X}^{1} \oplus \bar{\Omega}_{X}^{1}$. We are going to assign to $W$ a functional $\mathcal{F}_{W}(\varphi)$ on the space of fields.

There are the following elementary matrix-valued functionals on the space of fields:

(i) Each point $a \in X(\mathbb{C})$ provides a functional

$$
\mathcal{F}_{a}(\varphi):=\varphi(a) \in \operatorname{Mat}_{N}(\mathbb{C}) .
$$

(ii) A 1-form $\omega$ on $X(\mathbb{C})$ provides a functional

$$
\mathcal{F}_{\omega}(\varphi):=\int_{X(\mathbb{C})}\left[\varphi, d^{\mathbb{C}} \varphi\right] \wedge \omega \in \operatorname{Mat}_{N}(\mathbb{C})
$$

(iii) A pair of 1-forms $\left(\omega_{1}, \omega_{2}\right)$ on $X(\mathbb{C})$ provides a functional

$$
\mathcal{F}_{\left(\omega_{1}, \omega_{2}\right)}(\varphi):=\int_{X(\mathbb{C})} \varphi(x) \omega_{1} \wedge \omega_{2} \in \operatorname{Mat}_{N}(\mathbb{C})
$$

Let us choose a collection $\mathcal{P}$, possibly empty, of consecutive pairs $\left\{\omega_{j}^{i}, \omega_{j+1}^{i}\right\}$ of 1 -forms entering the cyclic word $W$. Take a field $\varphi$, and go along $W$, assigning to every element $\left\{a_{i}\right\}$ a matrix $\mathcal{F}_{a_{i}}(\varphi)$, to every 1 -form $\omega$ which does not enter to $\mathcal{P}$ a matrix $\mathcal{F}_{\omega}(\varphi)$, and to every pair of forms $\left\{\omega_{j}^{i}, \omega_{j+1}^{i}\right\}$ from the collection $\mathcal{P}$ a matrix $\mathcal{F}_{\left(\omega_{j}^{i}, \omega_{j+1}^{i}\right)}(\varphi)$. This way for a given collection $\mathcal{P}$ and a given field $\varphi$ we obtain a cyclic word in $\operatorname{Mat}_{N}(\mathbb{C})$, so its trace is well defined, providing a complex-valued functional, denoted by $\mathcal{F}_{W, \mathcal{P}}(\varphi)$. The functional $\mathcal{F}_{W}(\varphi)$ is obtained by taking the sum over all possible collections $\mathcal{P}$ :

$$
\mathcal{F}_{W}(\varphi)=\sum_{\mathcal{P}} \mathcal{F}_{W, \mathcal{P}}(\varphi)
$$

Example. Let $W=\mathcal{C}\left(\left\{s_{0}\right\} \otimes\left\{s_{1}\right\} \otimes \ldots \otimes\left\{s_{m}\right\}\right)$. Then we recover formula (34) for $\mathcal{F}_{W}(\varphi)$.

Given an integer $N$, we would like to define the correlator corresponding to $W$ via a Feynman integral

$$
\operatorname{Cor}_{X, N, h, \mu}(W):=\int \mathcal{F}_{W}(\varphi) e^{i S(\varphi)} \mathcal{D} \varphi
$$




$$
S(\varphi):=\frac{1}{2 \pi i} \int_{X(\mathbb{C})} \operatorname{Tr}\left(\frac{1}{2} \partial \varphi \wedge \bar{\partial} \varphi+\frac{1}{6} \hbar \cdot \varphi[\partial \varphi, \bar{\partial} \varphi]\right) .
$$

Observe that $S(\varphi)$ is real: $\overline{S(\varphi)}=S(\bar{\varphi})$. Unfortunately formula (296) does not have a precise mathematical sense. We understand it by postulating the perturbation series expansion with respect to a small parameter $\hbar$, using the standard Feynman rules, and then taking the leading term in the asymptotic expansion as $N \rightarrow \infty, \hbar=N^{-1 / 2}$. Before the implementation of this plan, let us recall the finite dimensional situation.

\subsection{Feynman rules}

This subsection surveys well-known results, mostly due to Feynman and t'Hooft, and is included for the convenience of the reader only.

Feynman rules for finite-dimensional integrals. We follow Wit, Section 1.3. Let $V$ be a finite dimensional real vector space and $B(v, v) \in S^{2}\left(V^{*}\right)$ a positive definite quadratic bilinear symmetric form on $V$. Denote by $d v$ an invariant Lebesgue measure on $V$ normalized by

$$
\int_{V} e^{-B(v, v) / 2} d v=1
$$

Let us consider a polynomial function on $V$ given by an expansion

$$
Q(v)=\sum_{m \geq 1} g_{m} Q_{m}(v) / m !, \quad Q_{m}(v) \in S^{m}\left(V^{*}\right)
$$

where $g_{m}$ are formal variables. Let $f_{i}(v) \in V^{*}$. Consider the following integral, the correlator of $f_{1}, \ldots, f_{N}$ :

$$
\left\langle f_{1}, \ldots, f_{N}\right\rangle:=\int_{V} f_{1}(v) \ldots f_{N}(v) e^{-B(v, v) / 2+Q(v)} d v \in \mathbb{C}\left[\left[g_{1}, g_{2}, \ldots\right]\right]
$$

where we expand $e^{Q(v)}$ into a series, and then calculate each term separately. This integral is computed using Feynman graphs as follows. Let $\mathbf{n}:=\left\{n_{1}, n_{2}, \ldots\right\}$ be any sequence of nonnegative integers which is eventually zero. Let $G(N, \mathbf{n})$ be the set of equivalence classes of graphs which have $N$ 1-valent vertices labeled by $1, \ldots, N$, and $n_{i}$ unlabeled $i$-valent vertices, $i \geq 1$. The labeled vertices are called external, and unlabeled ones internal vertices.

To each internal vertex $v$ of $\Gamma$ of valency $|v|$ we assign a tensor $Q_{|v|}$. To each external vertex labeled by $i$ we assign a vector $f_{i}$. Taking the product over all vertices of $\Gamma$, we get a vector

$$
\prod_{i=1}^{N} f_{i} \prod_{v} Q_{|v|}
$$

in the tensor product of $V^{*}$ 's over the set of all flags of $G$. Further, let $B^{-1} \in S^{2} V$ be the inverse form to $B$. To each edge $e$ of the graph $\Gamma$ we assign a tensor $B_{e}^{-1}$ called the propogator. Then there is a vector in the the tensor product of $V$ 's over the set of all flags, that is pairs (a vertex $v$, an edge $E$ incident to $v$ ), of $G$ :

$$
\prod_{\text {edges } e \text { of } \Gamma} B_{e}^{-1}
$$


Contracting these two vectors we get a complex number

$$
F_{\Gamma}:=\left\langle\prod_{\text {vertices } v \text { of } \Gamma} Q_{|v|}, \quad \prod_{\text {edges } e \text { of } \Gamma} B_{e}^{-1}\right\rangle \in \mathbb{C}
$$

where for external vertices $i$ we put $Q_{|i|}:=f_{i}$.

Theorem 13.1. a) One has

$$
\left\langle f_{1}, \ldots, f_{N}\right\rangle=\sum_{n} \prod_{i} g_{i}^{n_{i}} \sum_{\Gamma \in G(N, \mathbf{n})}|\operatorname{Aut}(\Gamma)|^{-1} F_{\Gamma}\left(f_{1}, \ldots, f_{n}\right)
$$

where $\operatorname{Aut}(\Gamma)$ denotes the group of automorphisms of $\Gamma$ which fix the external vertices.

b) One has

$$
\log \left\langle f_{1}, \ldots, f_{N}\right\rangle=\sum_{n} \prod_{i} g_{i}^{n_{i}} \sum_{\Gamma \in G_{0}(N, \mathbf{n})}|\operatorname{Aut}(\Gamma)|^{-1} F_{\Gamma}\left(f_{1}, \ldots, f_{n}\right)
$$

where the sum now is over the subset $G_{0}(N, \mathbf{n}) \subset G(N, \mathbf{n})$ of connected graphs only.

To deduce b) from a) observe that the sum in (297) is over all, possibly disconnected, diagrams. The automorphisms of a graph are given by the automorphisms of the connected components, and the permutation groups $S_{n}$ of $n$ identical copies of connected components. It remains to notice that

$$
\prod_{i} \sum_{j} \frac{F_{\Gamma_{i}}^{n_{j}}}{n_{j} !}=\prod_{i} \exp \left(F_{\Gamma_{i}}\right)=\exp \left(\sum_{i} F_{\Gamma_{i}}\right)
$$

Feynman rules for the matrix model (296). Let us explain how to write an asymptotic expansion in $\hbar$ for the Feynman integral (296). The quadratic form in our case is

$$
B(\varphi)=(2 \pi i)^{-1} \sum_{i, j=1}^{N} \frac{1}{2} \int_{X(\mathbb{C})} \partial \varphi_{j}^{i} \wedge \bar{\partial} \varphi_{i}^{j}=-(2 \pi i)^{-1} \sum_{i, j=1}^{N} \int_{X(\mathbb{C})} \varphi_{j}^{i} \cdot \partial \bar{\partial} \varphi_{i}^{j} .
$$

The vector space $V$ is the infinite-dimensional space of $\operatorname{Mat}_{N}(\mathbb{C})$-valued functions $\varphi$ on $X(\mathbb{C})$.

Let us assume first that $N=1$. Then the Laplacian $\Delta=(2 \pi i)^{-1} \bar{\partial} \partial$ has a one-dimensional kernel. So the inverse form $B^{-1}$ can be defined only on a complement to the kernel. Such a complement is described by a choice of a 2-current $\mu$ on $X(\mathbb{C})$ with a non-zero integral, which we normalize to be 1 . It consists of functions $\varphi$ orthogonal to $\mu$. The Green function $G_{\mu}(x, y)$ describes the bilinear form $B^{-1}$ on the complement as follows. The dual $V^{*}$ contains a dense subspace of smooth 2 -forms $\omega$ on $X(\mathbb{C})$. The value of the bilinear form on $\omega_{x} \cdot \omega_{y} \in S^{2} V^{*}$ is $\int_{X(\mathbb{C})^{2}} G_{\mu}(x, y) \omega_{x} \otimes \omega_{y}$. Its restriction to the subspace of $\omega$ 's orthogonal to constants does not depend on the ambiguity in the definition of $G_{\mu}(x, y)$.

Now let $N>1$. Let $e_{j}^{i}$ be an elementary matrix with the only non-zero entry, 1 , at the $(i, j)$ place. Then it is clear from (299) that the propogator $B^{-1}$ is given by

$$
B^{-1}=-G_{\mu}(x, y) \sum_{i, j=1}^{N} e_{j}^{i} \otimes e_{i}^{j} .
$$



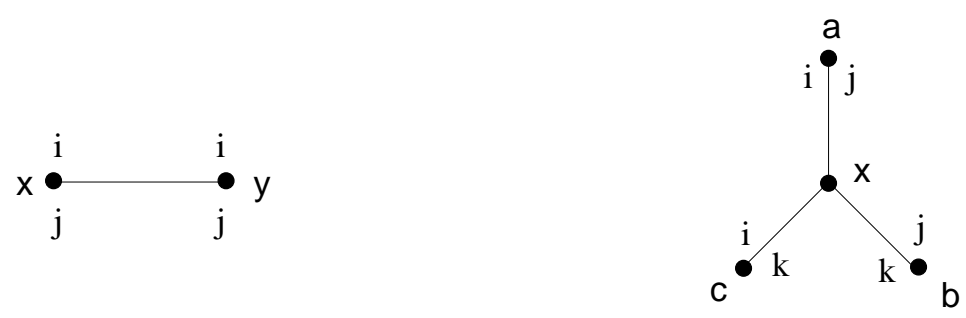

Figure 37: Feynman diagrams for the propagator and the vertex contribution

Its Feynman diagram is on the left of Fig 37. Recall the cubical term of the action $S(\varphi)$ :

$$
\hbar \cdot \sum_{i, j, k=1}^{N} \int_{X(\mathbb{C})}\left(\varphi_{j}^{i}(x) \partial \varphi_{k}^{j}(x) \wedge \bar{\partial} \varphi_{i}^{k}(x)-\varphi_{j}^{i}(x) \bar{\partial} \varphi_{k}^{j}(x) \wedge \partial \varphi_{i}^{k}(x)\right) .
$$

Recall a convenient normalisation of the maps $\omega_{n}$ introduced in (??) and denoted by $\omega_{n}^{*}$.

It follows that the contribution of the three valent vertex shown on the right of Fig 37 is

$$
\begin{gathered}
-\hbar \cdot \int_{X(\mathbb{C})}\left(G_{\mu}(a, x) \partial G_{\mu}(b, x) \wedge \bar{\partial} G_{\mu}(c, x)-G_{\mu}(a, x) \bar{\partial} G_{\mu}(b, x) \wedge \partial G_{\mu}(c, x)\right) \sum_{i, j, k=1}^{N} e_{j}^{i} \otimes e_{k}^{j} \otimes e_{i}^{k}= \\
\hbar \cdot \int_{X(\mathbb{C})} \omega_{2}^{*}\left(G_{\mu}(a, x) \wedge G_{\mu}(b, x) \wedge G_{\mu}(c, x)\right) \sum_{i, j, k=1}^{N} e_{j}^{i} \otimes e_{k}^{j} \otimes e_{i}^{k} .
\end{gathered}
$$

Integral (300) is convergent by Lemma 2.5. It does not change if we add a constant to $G_{\mu}$.

Recall that a ribbon graph is a graph with an additional structure: a cyclic order of the edges sharing each vertex. The contraction procedure for such propogator and vertex contributions implies that the non-zero contributions are given by ribbon graphs only (Fig [38). Each ribbon graph $\Gamma$ contributes a Feynman integral entering with a certain weight $w_{\Gamma}$, calculated below.

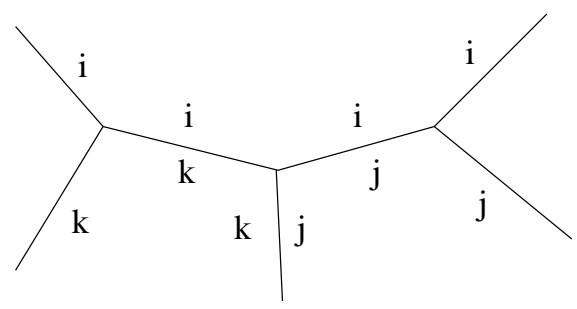

Figure 38: Non-zero contributions are given by ribbon graphs only.

A ribbon graph provides a surface $S_{\Gamma}$. It is obtained by taking a compact surface without boundary $\bar{S}_{\Gamma}$ and removing from it several discs. The graph $\Gamma$ is embedded into the surface $S_{\Gamma}$, so that the external vertices are on the boundary. It provides a decomposition of $\bar{S}_{\Gamma}-\Gamma$ into polygons, called faces. Let $f_{\Gamma}, e_{\Gamma}$ and $v_{\Gamma}$ be the number of polygons, edges and vertices of $\Gamma$. So each Feynman diagram $\Gamma$ contributes an integral with the weight $\omega_{\Gamma}=N^{f_{\Gamma}} \hbar^{v_{\Gamma}}-(m+1)$.

The Euler characteristic of the surface $\bar{S}_{\Gamma}$ is $\chi_{\Gamma}=v_{\Gamma}+f_{\Gamma}-e_{\Gamma}$. Let us shrink each boundary component of the surface $S_{\Gamma}$ into a point. The resulting surface is homeomorphic to $\bar{S}_{\Gamma}$. It 
has inside a ribbon graph with all vertices of valency 3 except the shrinked points, whose total valency is $m+1$. Thus $(m+1)+3 v_{\Gamma}=2 e_{\Gamma}$. So $\chi_{\Gamma}=f_{\Gamma}-v_{\Gamma} / 2-(m+1) / 2$.

\subsection{Hodge correlators and the matrix model}

Definition 13.2. The correlator $\operatorname{Cor}_{X, \mu}(W)$ is the leading term of the asymptotics of $\operatorname{Cor}_{X, N, \mu}(W)$ as $N \rightarrow \infty$ and $\hbar=N^{-1 / 2}$.

The number of ribbon graphs decorated by a given cyclic word $W$, even if we restrict our attention to connected diagrams, is infinite: one could have a surface $S_{\Gamma}$ of arbitrary genus, and even if the genus is zero, one could have as many loops in $\Gamma$ as we want. However Lemma 13.3 tells that all but finitely many of them give zero contribution to $\operatorname{Cor}_{X}(W)$. Precisely, only trees will contribute, and, of course, there are finitely many trees with given external vertices.

Lemma 13.3. The logarithm $\log \operatorname{Cor}_{X, \mu}(W)$ of the Feynman integral correlator is the sum of integrals assigned to connected Feynman diagrams, which are plane trees.

Proof. Since $\hbar=N^{-1 / 2}$, the finite-dimensional integral corresponding to a Feynman diagram $\Gamma$ enters with the weight $\omega_{\Gamma}=N^{f_{\Gamma}-v_{\Gamma} / 2+(m+1) / 2}=N^{(m+1)+\chi_{\Gamma}}$. Here $m$ is fixed by the cyclic word $W$. Thus the leading term of the asymptotics comes from the Feynman diagrams $\Gamma$ with the minimal possible value of $\chi_{\Gamma}$, that is plane trees.

Theorem 13.4. The logarithm $\log \operatorname{Cor}_{X, \mu}(W)$ of the Feynman integral correlator equals, up to a sign computed below, to the value of the Hodge correlator $\operatorname{Cor}_{\mathcal{H}}^{*}(W)$ on $W$.

Proof. We need to show that the contribution of a $W$-decorated plane trivalent tree $T$ to the Hodge correlator is the same as the finite dimensional Feynman integral $\varphi_{T}$ assigned by the Feynman rules to the Feynman diagram corresponding to $T$. The contribution to $\varphi_{T}$ of a 3 -valent vertex $v$ of $T$ is $N^{3}$ times the integral

$$
\begin{gathered}
-\int_{X(\mathbb{C})}\left(G_{\mu}(a, x) \partial G_{\mu}(b, x) \wedge \bar{\partial} G_{\mu}(c, x)+G_{\mu}(a, x) \bar{\partial} G_{\mu}(b, x) \wedge \partial G_{\mu}(c, x)\right)= \\
\int_{X(\mathbb{C})} \omega_{2}^{*}\left(G_{\mu}(a, x) \wedge G_{\mu}(b, x) \wedge G_{\mu}(c, x)\right) .
\end{gathered}
$$

Integral (301) is skewsymmetric under the permutations of $(a, b, c)$. Indeed, it is evidently skewsymmetric under $b \leftrightarrow c$. To check skewsymmetry under $a \leftrightarrow b$, notice that the skewsymmetrisation leads to a complete derivative:

$$
f(\partial g \wedge \bar{\partial} h)-f(\partial h \wedge \bar{\partial} g)+g(\partial f \wedge \bar{\partial} h)-g(\partial h \wedge \bar{\partial} f)=\partial(f g \bar{\partial} h)+\bar{\partial}(f g \partial h) .
$$

Let us use this symmetry to find a preferred presentation for the integrand of the Feynman integral $\varphi_{T}$ related to $T$.

Recall that a flag at a vertex $v$ is a pair (the vertex $v$, an edge incident to $v$ ). So each internal vertex $v$ of $T$ gives rise to three flags. We say that among the three flags $(x, a),(x, b),(x, c)$ relevant to integral (301) the flag $(x, a)$ is the free flag at the vertex $x$ - it contributes the Green function $G_{\mu}(a, x)$ rather then its derivative. Thanks to the symmetry, for any 3 -valent vertex $v$ of $T$, in the contribution to the Feynman integral $\varphi_{T}$ we can make any flag incident to $v$ to be the free flag. Choose an external edge of $T$, called the root edge. Then for each internal vertex there is a unique flag which is the closest to the root. We call it the upper flag. Using the symmetry of the integral (301) one easily proves the following: 
Lemma 13.5. Given a root edge of $T$, there is a unique presentation of the integrand for the integral $\varphi_{T}$ for which the set of free flags is the set of upper flags.

On the other hand this contribution can be written as

$$
2^{m-1} \int_{X(\mathbb{C})^{m-1}} G_{0}\left(\partial G_{1} \wedge \bar{\partial} G_{2}\right) \wedge \ldots \wedge\left(\partial G_{2 m-3} \wedge \bar{\partial} G_{2 m-2}\right)
$$

where the integration is over the copies of $X$ assigned to the internal vertices of $T$, and $\left(G_{2 k+1}, G_{2 k+2}\right)$ is the pair of Green functions assigned to the pair of non-upper edges of $k$-th vertex of $T$. We claim that

$$
\left(\underline{302)}=(-1)^{(m-1)(m-2) / 2} \int_{X(\mathbb{C})^{m-1}} \omega_{2 m-2}^{*}\left(G_{0}, \ldots, G_{2 m-2}\right) .\right.
$$

By the very definition, the integrands coincide up to a numerical coefficient. It is easy to see that the coefficient must be \pm 1 .

A generalization of the Feynman integral. Let $\mathcal{G}$ be a Lie algebra with an invariant symmetric bilinear form $Q: \mathcal{G} \otimes \mathcal{G} \longrightarrow \mathbb{C}$. Then there is a generalisation of the Lagrangian for $\operatorname{Mat}_{N}(\mathbb{C})$ defined as follows. Consider $\mathcal{G}$-valued fields $\{\varphi\}$ on $X(\mathbb{C})$. Then there is an action

$$
S_{\mathcal{G}}(\varphi):=\frac{1}{2 \pi i} \int_{X(\mathbb{C})} Q\left(\frac{1}{2} \partial \varphi \wedge \bar{\partial} \varphi+\frac{1}{6} \hbar \cdot \varphi \otimes[\partial \varphi, \bar{\partial} \varphi]\right)
$$

Here the expression in parenthesis is a $\mathcal{G} \otimes \mathcal{G}$-valued 2-form on $X(\mathbb{C})$, so applying the bilinear form $Q$ we get a 2 -form and integrate it over $X(\mathbb{C})$. Formulas (293)-(294) provide $\mathcal{G}$-valued functionals on the space of fields corresponding to a choice of a point $a$, a 1-form, and a pair of 1 -forms on $X(\mathbb{C})$. Repeating verbatim our construction we assign to a cyclic word $W$ a correlator

$$
\operatorname{Cor}_{X, \mathcal{G}, h}(W):=\int \mathcal{F}_{W}(\varphi) e^{i S_{\mathcal{G}}(\varphi)} \mathcal{D} \varphi
$$

In the case when $\mathcal{G}=\operatorname{Mat}_{N}$, we recover the Feynman integral (296).

The motion equation for the action is $\delta S_{\mathcal{G}}(\varphi) / \delta \varphi=0$. Renormalising $\varphi \longmapsto \varphi / \hbar$ to remove dependence on $\hbar$, we get $\bar{\partial} \partial \varphi=[\bar{\partial} \varphi, \partial \varphi]$.

\section{References}

[B] Beilinson A.A.: Regulators and values of L-functions. (Russian) Current problems in mathematics, Vol. 24, 181-238, Itogi Nauki i Tekhniki, Akad. Nauk SSSR, Vsesoyuz. Inst. Nauchn. i Tekhn. Inform., Moscow, 1984.

[B1] Beilinson A.A.: Height pairings between algebraic cycles. $K$-theory, arithmetic and geometry (Moscow, 1984-1986), 1-25, Lecture Notes in Math., 1289, Springer, Berlin, 1987.

[B2] Beilinson A.A.: Higher regulators of modular curves. Contemp. Math. 55, 1-34 (1986)

[BD] Beilinson A.A., Deligne P, Interprétation motivique de la conjecture de Zagier. in Symp. in Pure Math., v. 55, part 2, 1994, 
[BL] Beilinson A.A., Levin A.M. : The elliptic polylogarithms, Proc. Symp. in Pure Math, vol. 55, (1994), part 2, 126-196.

[BD] Beilinson A.A., Deligne P. Unpublished preprint on motivic polylogarithms.

[BK] Bloch S., Kato K.: L-functions and Tamagawa numbers of motives. Grothendieck Festschrift, Vol 1, 333-400. Progress Math. 86, Birkhäuser, Boston (1990)

[Bo1] Borel A.: Cohomologie des espaces fibrés principaux, Ann. Math. 57 (1953), 115-207.

[Bo2] Borel A.: Cohomologie de $S L_{n}$ et valeurs de fonctions zêta aux points entiers. Annali Scuola Normale Superiore Pisa 4 (1977), 613-636.

[Br] Brown F.: Mixed Tate motives over $\mathbb{Z}$. arXiv:1102.1312.

[CKS] Cattani E., Kaplan A., Schmid W.: Degeneration of Hodge structures Annals of Math. 123, (1986), 457-535.

[Ch] Chen, Kuo Tsai: Iterated path integrals. Bull. Amer. Math. Soc. 83 (1977), no. 5, 831-879.

[D] Deligne P.: Hodge theory II. Publ. IHES No. 40 (1971), 5-57;

[D1] Deligne P.: Le group fondamental de la droite projective moine trois points, In: Galois groups over $\mathbb{Q}$. Publ. MSRI, no. 16 (1989) 79-298.

[D2] Deligne P.: Structures des Hodges mixte réeles. Proc. Symp. in Pure Math, vol. 55, (1994), part 1, 509-516.

[DG] Deligne P., Goncharov, A. Groupes fondamentaux motiviques de Tate mixte. Ann. Sci. Ecole Normale Sup. math.NT/0302267.

[Den1] Deninger Ch.: Higher regulators and Hecke L-series of imaginary quadratic fields II, Ann. of Math, 132, N1 (1990), 131-158.

[DS] Deninger Ch. Scholl, A.: The Beilinson conjectures. L-functions and arithmetic (Durham, 1989), 173-209, London Math. Soc. Lecture Note Ser., 153, Cambridge Univ. Press, Cambridge, 1991.

[Dr1] Drinfeld V. G.: On quasitriangular quasi-Hopf algebras and on a group that is closely connected with $\operatorname{Gal}(\bar{Q} / Q)$. Algebra i Analiz 2 (1990), no. 4, 149-181; Leningrad Math. J. 2 (1991), no. 4, 829-860.

[Dr2] Drinfeld V. G.: Elliptic modules. Mat. Sb. (N.S.) 94(136) (1974), 594-627.

[Dr3] Drinfeld, V. G.: Two theorems on modular curves, Functional analysis and its applications. $7(2): 83-84$.

[GK] Ginzburg V.; Kapranov M.: Koszul duality for operads. Duke Math. J. 76 (1994), no. 1, 203-272.

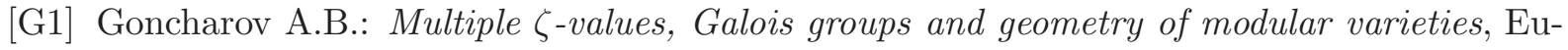
ropean Congress of Mathematics, Vol. I (Barcelona, 2000), 361-392, Progr. Math., 201, Birkhauser, Basel. math.AG/0204102. 
[G2] Goncharov A.B.: Chow polylogarithms and regulators, Math. Res. Letters, vol. 2. (1995), N 1, pp. 95-112.

[G3] Goncharov A.B.: Mixed elliptic motives. Galois representations in Arithmetic Algebraic geometry, London Math. Soc. Lect. Note Ser., vol. 254, Cambridge University Press, 1998. $147-221$.

[G4] Goncharov A.B.: The dihedral Lie algebras and Galois symmetries of $\pi_{1}^{(l)}\left(\mathbb{P}^{1} \backslash\left\{0, \mu_{N}, \infty\right\}, v_{1}\right), \quad$ Duke $\quad$ Math. $\quad$ J., $\quad$ vol $100, \quad$ N3, $\quad$ (2001), pp. $397-487$. math.AG/0009121.

[G5] Goncharov A.B.: Galois groups, geometry of modular varieties and graphs. Proc. of Arbeitstagung, June 1999, in the MPI preprint (http//www.mpim-bonn.mpg.de/).

[G6] Goncharov A.B.: Euler complexes and geometry of modular varieties. GAFA (2008). Special volume dedicated to J.Bernstein's 60th birthday. arXive: math/0510310.

[G7] Goncharov A.B.: Galois symmetries of fundamental groupoids and noncommutative geometry. Duke Math. J. 128 (2005), no. 2, 209-284, ArXive math.AG/0208144,

[G8] Goncharov A.B.: Polylogarithms, regulators, and Arakelov motivic complexes. JAMS (2005), N1, 1-60.

[G9] Goncharov A.B.: Multiple polylogarithms, cyclotomy and modular complexes. Math. Res. Lett. 5 (1998), no. 4, 497-516

[G10] Goncharov A.B.: Polylogarithms and motivic Galois groups Motives, Vol 2, (1995).

[GII] Goncharov A.B.: Hodge correlators II. Moscow Math Journal. Special issue dedicated to Deligne's 65th birthday. N1, 2010, 139-188. arXiv:0807.4855.

[GZ] Goncharov A.B., Zhao J.: The Grassmannian trilogarithm. Compositio Math. 127 (2001), no. $1,83-108$.

[H] Hain R.: The geometry of the mixed Hodge structure on the fundamental group. Algebraic geometry, Bowdoin, 1985, 247-282, Proc. Sympos. Pure Math., 46, Part 2, Amer. Math. Soc., Providence, RI, 1987.

[H1] Hain R.: Classical polylogarithms. Proceedings of Symp. in Pure Math., Volume 55, (1994), vol. $2,3-42$.

[HZ] Hain R, Zucker S.: Unipotent variations of mixed Hodge structure. Invent. Math. 88 (1987), no. $1,83-124$.

[HW] Huber A., Wildeshaus J. : Classical Motivic Polylogarithm according to Beilinson and Deligne. Documenta Mathematica 3: 27-133, 1998.

[Ka] Kapranov M.: Real mixed Hodge structures. Preprint 2008, arXiv:0802.0215.

[KM] Kashiwara M.: A study of variation of mixed Hodge structure. Publ. Res. Inst. Math. Sci. 22 (1986), no. 5, 991-1024. 
[K] Kontsevich M.: Formal (non) commutative symplectic geometry. The Gelfand mathematical seminars, Birkhauser, 1993, p. 173-187.

[K2] Kontsevich M.: Operads and motives in deformation quantization. Lett. Math. Phys., 48 (1999) N1, 35-72.

[L] Levin A.: Variations of $\mathbb{R}$-Hodge-Tate structures. Preprint MPI-2001.

[Le] Levine M.: Mixed motives. American Mathematical Soc., 1998.

[Le2] Levine M.: Tate motives and the vanishing conjectures for algebraic K-theory. In Algebraic K-theory and Algebraic topology, 167-188, NATO Adv. Sci. Inst. Ser. C Math. Phys. Sci. 407 Kluver (1993).

[Li] Lichtenbaum S.: The Weil-Etale Topology for Number Rings. arXiv:math/0503604

[LV] Loday J.-L., Vallette B.: Algebraic operades. http://math.unice.fr/ brunov/Operads.pdf.

[La] Lang S. Introduction to Arakelov theory. Springer Verlag. 1988.

[Ma] Manin, Ju. I.: Parabolic points and zeta functions of modular curves, Izvestiya Akademii Nauk SSSR. Seriya Matematicheskaya 36: 19-66,

[SS] Schappacher N., Scholl A.: Beilinson's theorem on modular curves. Beilinson's conjectures on special values of $L$-functions, 273-304, Perspect. Math., 4, Academic Press, Boston, MA, 1988.

[S] Suslin A.A.: $K_{3}$ of a field and Bloch's group, Proceedings of the Steklov Institute of Mathematics 1991, Issue 4.

[M] Morgan J.: The algebraic topology of smooth algebraic varieties. Inst. Hautes tudes Sci. Publ. Math. No. 48 (1978), 137-204.

[Z1] Zagier D.: Polylogarithms, Dedekind zeta functions and the algebraic K-theory of fields. Arithmetic algebraic geometry (Texel, 1989), 391-430, Progr. Math., 89, Birkhuser Boston, MA, 1991.

[V] Voevodsky V.: Triangulated categories of motives over a field. In Cycles, transfers, and motivic homology theories, volume 143 of Ann. of Math. Stud., pages 188-238. Princeton Univ. Press, Princeton, NJ, 2000.

[We] Weil A.: Elliptic functions according to Eisenstein and Kronecker. Ergebnisse der Mathematik, 88, Springer 1977.

[Wit] Witten E.: Perturbative quantum field theory. In: Quantum fields and Strings: A course for mathematicians. Volume 1. P. Deligne, ... editors. AMS (1999). 
$a \bullet x \quad y \quad b$ 